\title{
Cyclic di-nucleotide monophosphate cyclase in Firmicutes: from basic to practical approach
}

\author{
Dissertation \\ for the award of the degree \\ "Doctor rerum naturalium” \\ of the Georg-August-Universität Göttingen
}

\author{
Within the Binational Doctoral Program \\ on Molecular Biosciences and Biomedicine \\ Georg-August-Universität School of Science (GAUSS) \\ Universidad Nacional de Rosario (UNR)
}

Submitted by

Ingrid M. Quintana

From Rosario (Argentina)

Rosario-Göttingen, 2018 


\section{Thesis Committee}

Dr. Christian Magni

(Instituto de Biología Molecular y Celular de Rosario, IBR CONICET)

Prof. Dr. Jörg Stülke

(Institute of Microbiology and Genetics, Department of General Microbiology)

Dr. Hugo Gramajo

(Instituto de Biología Molecular y Celular de Rosario, IBR CONICET)

\section{Members of the Examination Board}

Dr. Hugo Gramajo

(Instituto de Biología Molecular y Celular de Rosario, IBR CONICET)

Dr. Raúl Raya

(Centro de Referencia para Lactobacilos, CERELA CONICET)

Prof. Dr. Gerhard Braus

(Institute for Microbiology and Genetics, Georg-August-Universität)

Date of oral examination: June $11^{\text {th }}, 2018$ 
Herewith I declare that I have written the doctoral thesis entitled "Cyclic di-nucleotide monophosphate cyclase in Firmicutes: from basic to practical approach” on my own and with no other sources and aids than quoted.

Ingrid M. Quintana 


\section{Acknowledgements}

I would like to thank in the first place my director Dr. Christian Magni and the whole LAB laboratory. It has been seven years of very hard work and extensive learning, surrounded by many people teaching me about science, partnership and teaching. Thanks Martin and Victor for your help, thanks to all the people I have met because of the lab. Very special thanks to Gabriela Martino, my science sister, who was my daily demonstration that teamwork and support is always possible.

I would also like to express my sincere gratitude to Facultad de Ciencias Bioquímicas y Farmacéuticas and the Universidad Nacional de Rosario. Free and lay tuition is something I will always be grateful for.

Natürlich möchte ich auch meinem Co-Direktor Prof. Dr. Jörg Stülke dafür danken, dass er mir die Möglichkeit gegeben hat, in einem so hervorragenden Labor zu arbeiten. Ebenfalls möchte ich PD Dr. Fabian Commichau und der gesamten Bacillus - Listeria - Mycoplasma Gruppe meinen Dank aussprechen; ein großartiger Motor, bei dem jede Verzahnung perfekt sitzt und arbeitet; diese gewonnenen Erfahrungen haben mein Leben verändert. Vielen Dank, Jan Gundlach, für die Hilfe bei meinen ersten Schritten in der c-di-AMP Welt und Christina Herzberg für Ihre Empfänglichkeit und Ihre experimentellen Ratschläge. Danken möchte ich außerdem Zuzanna Grubek, Jan Kampf, Cedric Blötz, Miriam Dormeyer, Johannes Gibhardt und Raphael Michna. Ich freue mich sehr, dass ich Sie getroffen habe und bin äußerst dankbar für Ihre Hilfe. Von Ihnen allen habe ich viel gelernt.

Special thanks to Nora Cascante-Estepa; no one has ever helped me so much, so selflessly. Silvia Castellon and Blanca Rincón, thank you too, for every conversation we had made my days brighter. Thanks to my Argentinian sister in Göttingen, Mariela Escobar, for every moment, trip, mate we shared.

I would also like to thank CONICET, ANPCYT, FBIOYF and DAAD for the financial support, as well as the Georg-August-Universität, and the staff from GAUSS, the CUAA$\mathrm{DAHZ}$ and the Binational PhD program for all the assistance along the way.

Thanks to the magic of true friends, for putting me back on track in difficult moments and for sharing the happiness in good ones. Thanks to the University, El Poli, Folk and life itself for surruonding me with such wonderful human beings. I feel truly fortunate when I think I couldn’t mention you all because I would need another thesis. My very special thanks to 
Acrolove and all the acroyoga Kula, evidence supporting I have family around the globe. Thank you for putting my head upside down, a smile in my face and siblings by my side: you restored my balance when the thesis was too heavy on my shoulders.

Gracias a mi familia, a mis padres y hermanos, cómo poner en palabras el idioma del corazón. Late por ustedes.

And last, but of course not least, gracias, thank you, vielen vielen Dank Chris, for your constant love, support and company, no matter what. 


\section{Table of contents}

Table of contents ................................................................................................................................ 1

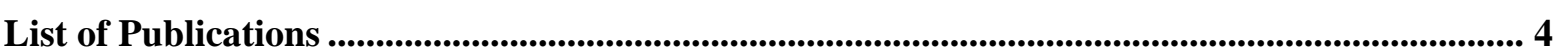

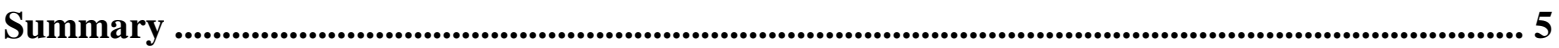

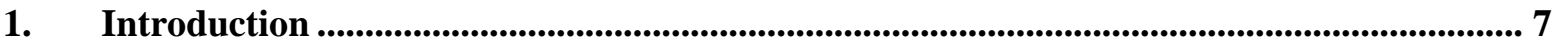

1.1. Lactic Acid Bacteria ..................................................................................................................

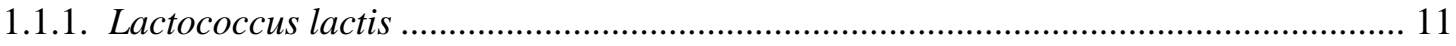

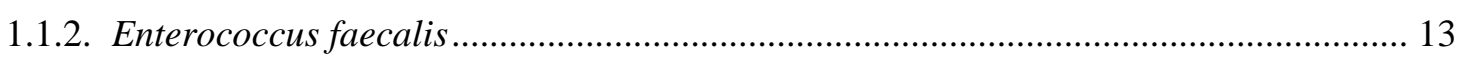

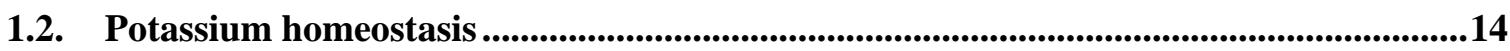

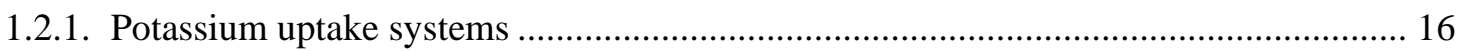

1.3. (3',5') cyclic-di-adenosine-monophosphate.............................................................................17

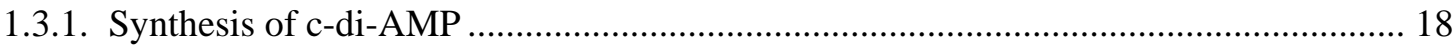

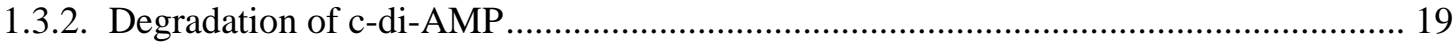

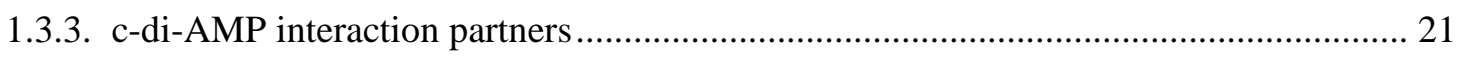

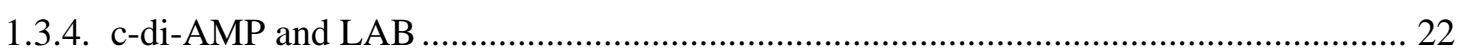

1.4. American Trypanosomiasis (Chagas disease) ...............................................................24

1.4.1. Chagas prevention ...................................................................................................... 26

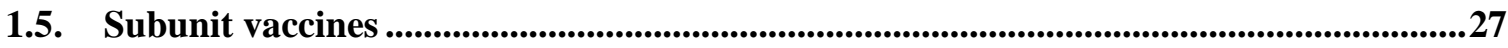

1.5.1. L. lactis in the industry: its potential as delivery vector ................................................ 29

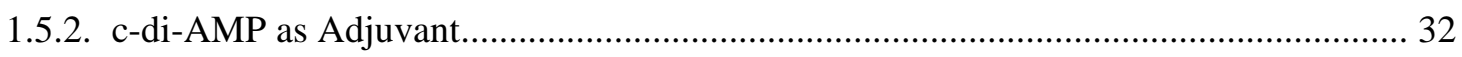

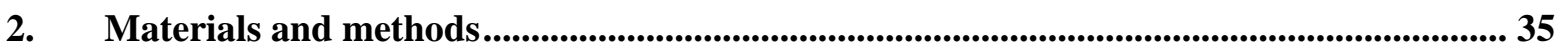

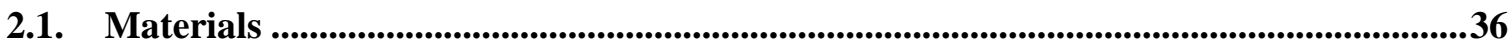

2.2. Strains and plasmids .............................................................................................................36

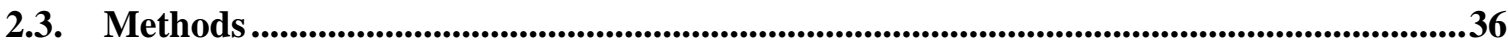

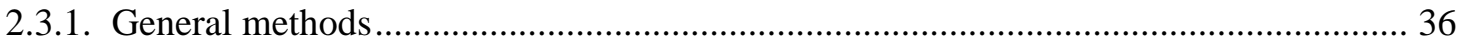

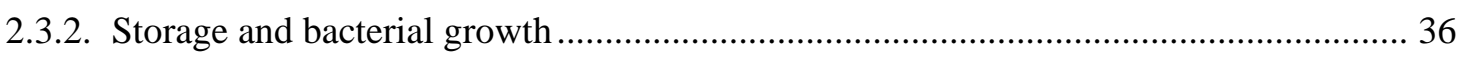

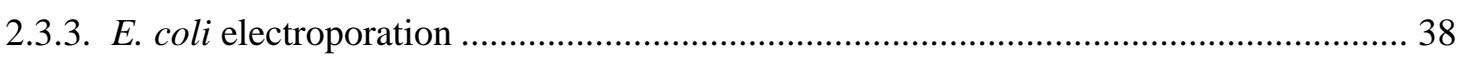

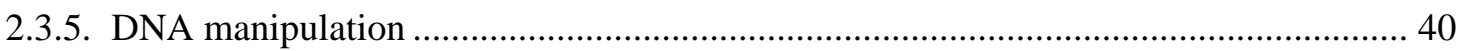

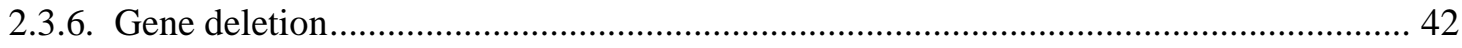

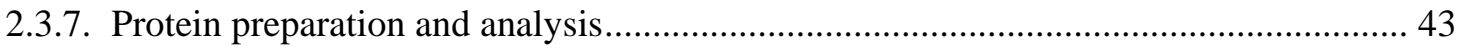

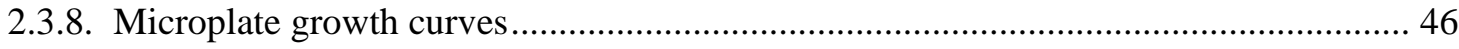

2.3.9. Protein pull-down experiment with strep-tagged magnetic beads................................. 50

2.3.10.Differential radial capillary action of ligand assay (DrACALA) ………........................ 50

2.3.11.Determination of c-di-AMP intracellular levels ............................................................ 51

2.3.12.Bis(p-nitrophenyl)phosphate assay. ............................................................................... 51

2.3.13.Light microscopy …………………………................................................................. 52 


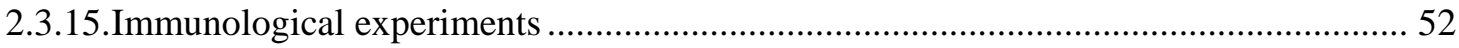

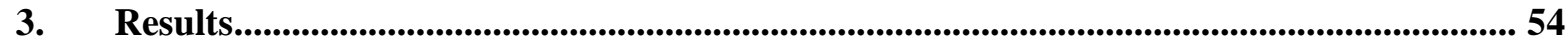

3.1. c-di-AMP and potassium uptake in L. lactis IL1403 ...........................................................55

3.1.1. Identification of c-di-AMP interaction partners in L. lactis IL1403 .............................. 55

3.1.2. c-di-AMP specific interaction to different Lactococcal proteins .................................. 59

3.1.3. KupA and KupB of L. lactis 1403 restore growth of E. coli LB650 .............................. 61

3.1.4. KupA and KupB are high affinity transporters ............................................................. 63

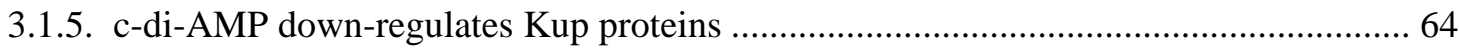

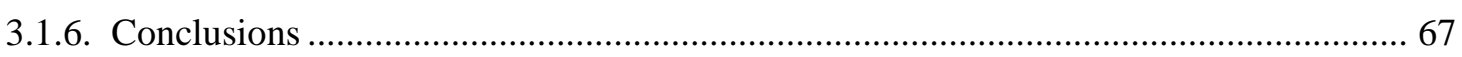

3.2. Studies on c-di-AMP synthesizing and degrading enzymes in L. lactis IL1403 .............69

3.2.1. Strain engineering for modification of intracellular c-di-AMP levels........................... 69

3.2.2. Phenotypic analysis of the strains developed ........................................................... 72

3.2.3. Intracellular levels of c-di-AMP are modified in strains L. lactis LL03-LL08 .............. 79

3.2.4. Phosphodiesterases c-di-AMP in L. lactis ...................................................................... 82

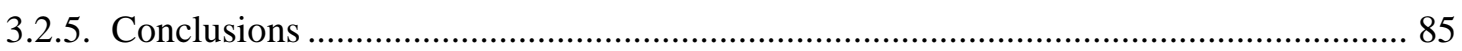

3.3. Development of an oral subunit vaccine prototype against Chagas disease ..................87

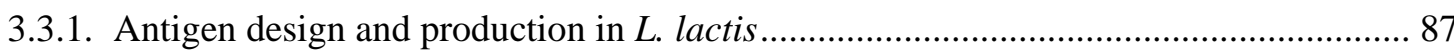

3.3.2. Immune response after mucosal co-administration of $L$. lactis strains producing TScf

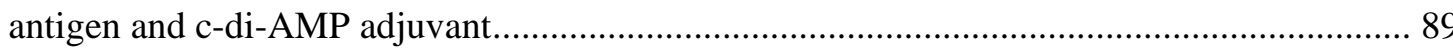

3.3.3. A single engineered L. lactis strain producing c-di-AMP antigen and TScf adjuvant: a

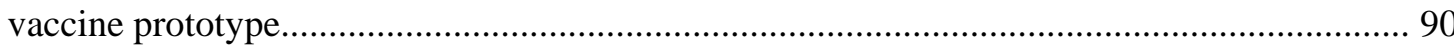

3.3.4. Immune response of engineered $L$. lactis co-expressing antigen and adjuvant after

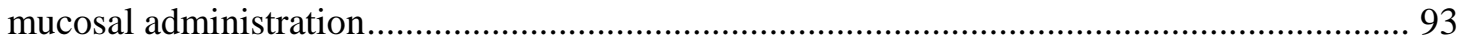

3.4. An approach into c-di-AMP metabolism in E. faecalis JH2-2 .......................................95

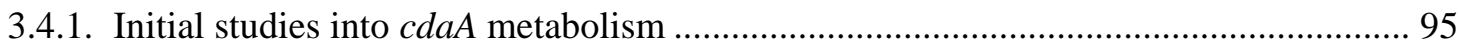

3.4.2. $\quad$ gdpP mutant in E. faecalis $\mathrm{JH} 2-2$........................................................................ 97

3.4.3. Virulence analysis of $g d p P$ mutant strain E. faecalis JH2-2 using the greater wax moth

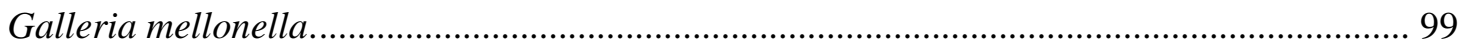

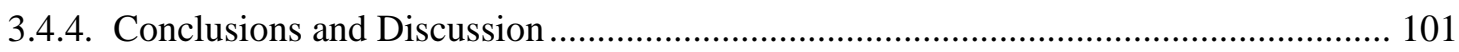

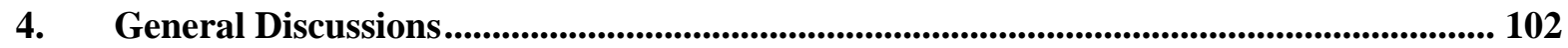

4.1. Novel potassium transporters in L. lactis IL1403 _..............................................................103

4.2. An updated overview of c-di-AMP in L. lactis IL1403 ......................................................104

4.3. L. lactis + c-di-AMP: a novel vaccine delivery system ....................................................106

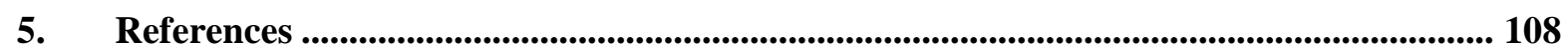

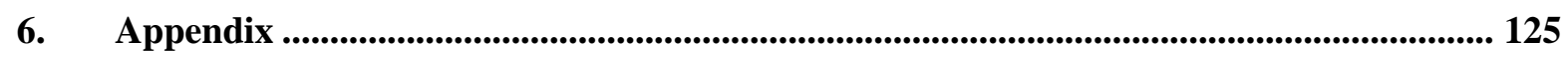

6.1. Peptide identification after c-di-AMP pull-down experiment.......................................126

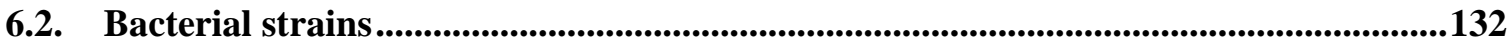

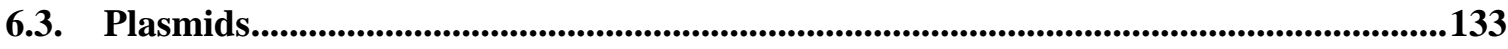




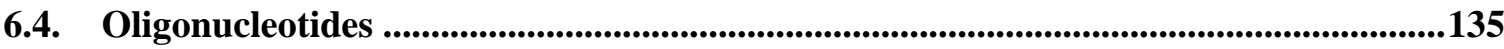

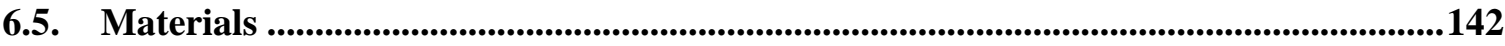

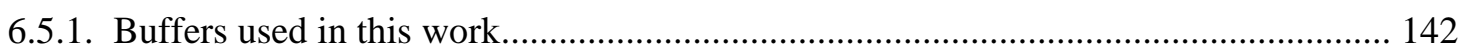

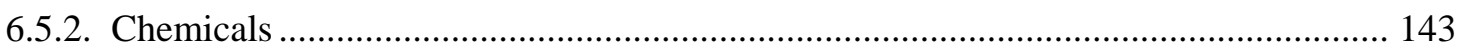

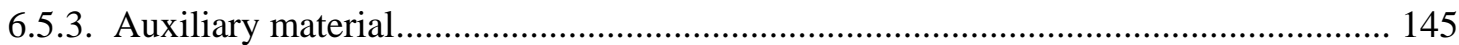

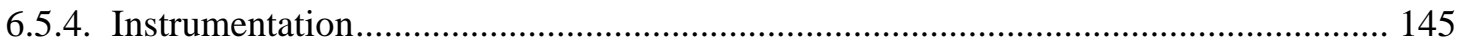

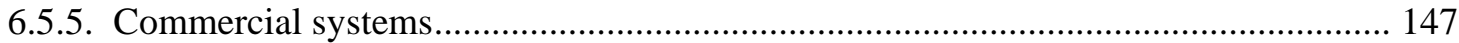

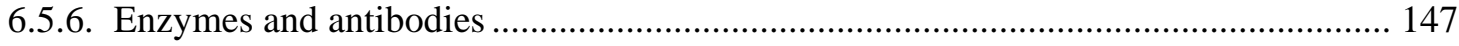

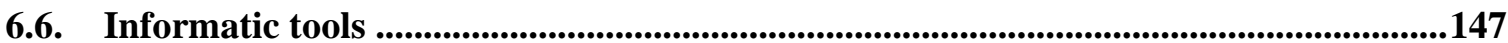

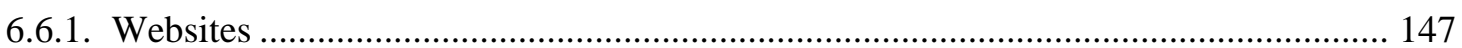

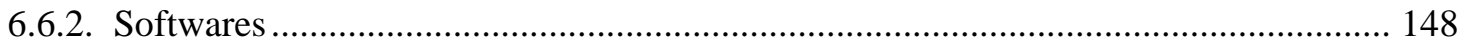

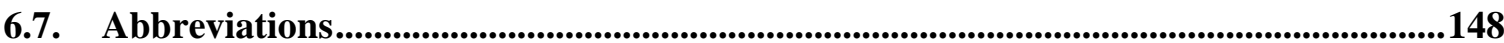

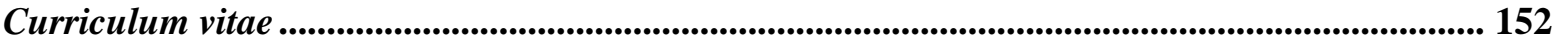




\section{List of Publications}

- “c-di-AMP levels in Lactococcus lactis: overexpression of cdaA and gdpP can significantly modify this second messenger intracellular pool.” Congress: Reunión conjunta de sociedades de biociencias. Noviembre, 2017. Buenos Aires, Argentina

- "The KupA and KupB proteins of Lactococcus lactis IL1403 are c-di-AMP receptor proteins responsible for potassium uptake.” Quintana I., Grubek Z., Gibhardt J., Lee V. T., Commichau F. M., Stülke J., and Magni C. (2018) Applied and Environmental Microbiology. Sent.

- "Genetic engineering of Lactococcus lactis co-producing antigen and the mucosal adjuvant $3^{\prime} 5^{\prime}$ - cyclic di adenosine monophosphate (c-di-AMP) to design a mucosal vaccine prototype.” Quintana I., Espariz M., Stülke J., Villar S., González F., Pacini F., Cabrera G., Bontempi I., Procheto E., Perez A. R., Marcipar I., Blancato V., and Magni C. (2018) Frontiers in Microbiology. Sent. 


\section{Summary}

Cyclic di-adenosine monophosphate (c-di-AMP) is a second messenger involved in diverse metabolic processes such as cell wall homeostasis, biofilm formation, antibiotics and heat resistance, among others. In Lactococcus lactis and Enterococcus faecalis, Lactic Acid Bacteria used not only as research models but also as a cell factory in biotechnological processes, the only reported interaction partner of c-di-AMP is the pyruvate carboxylase enzyme, PyrCarb. Nevertheless, in the last year investigations directed its main role towards potassium metabolism.

In this thesis, KupA and KupB, two potassium transporters encoded in L. lactis IL1403 genome, are described for the first time. According to an in silico analysis, these proteins, which belong to the Kup/HAK/KT family, are highly conserved in this species, being therefore a strain independent potassium uptake system. In addition, evidence shows that both proteins are able to uptake this cation with high affinity, and we demonstrate that KupA as well as KupB bind to and are down-regulated by c-di-AMP.

On the other hand, different strains derived from L. lactis IL1403 were developed aiming to modify intracellular pools of c-di-AMP in a stable system. One strategy for the reduction of c-di-AMP levels was the obtaining of $\triangle g d p P$ mutants via homologous recombination. Maintenance of this second messenger levels close to wild type ones, suggested the presence of another c-di-AMP degrading enzyme. A first description of a putative enzyme with this activity, encoded by yheB gene was done by BNPP assay. In addition, by use of a pH inducible vector, construction of strain L. lactis LL03 with concentrations of this second messenger above 15 times basal levels was possible. This system was therefore selected for further investigations on the development of a vaccine prototype against Chagas disease.

On the other hand, L. lactis is a promising candidate for the development of mucosal vaccines with more than 20 years of experimental research. Moreover, c-di-AMP has been reported as a strong mucosal adjuvant promoting both humoral and cellular immune responses. Altogether, in this thesis the development of a recombinant L. lactis strain is reported, able to produce both an antigen as well as an adjuvant in order to develop a novel vaccine prototype against the Trypanosoma cruzi parasite, the causal agent of Chagas disease. This is a tropical disease originated in a specific area of South America but currently spreading in four continents. 
Finally, an initial approach was done on c-di-AMP metabolism in E. faecalis. The presence of a Kup transporter was also corroborated in this species, and some basic characteristics of the c-di-AMP degradative pathway were explored via a $\Delta g d p P$ mutant construction. Finally, the impact of GdpP on the virulence of E. faecalis was analyzed by use of the infection model Galleria mellonella. 


\section{Introduction}

"Remember to look up at the stars and not down at your feet. Try to make sense of what you see and wonder about what makes the universe exist" Stephen Hawking 


\subsection{Lactic Acid Bacteria}

Along the course of history, human beings always sought for efficient ways of improving quality of everyday life. When food supplies were not round the corner, one of these ways concerned food preservation. In this sense, a wide variety of products including meat, cheese, vegetables and dairies to name a few, were well conserved thanks to the process of fermentation. Successful results were not investigated, there was a lot of variation from one fermented product to the next one and optimization of these procedures was obtained after trial and error. A widely practiced technique consisted on separating a small portion of a successful batch of a fermented product and using it to start the process in fresh food. This "back-slopping” method allowed people to maintain food in good conditions, by transferring the microorganisms which made fermentation possible. In lactic fermentation, the most represented amount of bacteria conforming the inocula were Lactic Acid Bacteria (LAB) (1) .

Nowadays, mechanisms of preservation are thoroughly understood and different combinations of LAB are used to inoculate a culture, expecting specific features in the final fermented product. In the cheese making industry, for instance, certain strains are used as starter or adjunct cultures to contribute to flavor and aroma development, or to other specific organoleptic features such as the formation of eyes via $\mathrm{CO}_{2}$ release (2) . Also, proteolysis and lipolysis are key pathways for texture and flavor improvement and so is the formation of C4 aroma compounds like acetoin and diacetyl, both derivatives of lactic acid (3).

The group of LAB cannot be addressed as microorganisms falling in one simple definition. Instead, they are referred to as a group of bacteria with certain key characteristics in common. Principally, the high amounts of lactic acid they produce when grown in the presence of a suitable carbon source, as result of homofermentation or heterofermentation, in which case they produce acetic acid and ethanol as well. These acidic compounds and the concomitant decrease in $\mathrm{pH}$ are one of the main reasons for the growth inhibition of undesired microorganisms. Moreover, LAB have less than $50 \%$ of $\mathrm{G}+\mathrm{C}$ content in their genomes and they are non-sporulating Gram-positive bacteria. They are strictly fermentative, anaerobic or aerotolerant and morphologically they can be cocci or rod shape bacteria. Phylogenetically, LAB genera are all included in the firmicutes phylum, except for the Bifidobacterium genus, belonging to Actinobacteria. Genera within Firmicutes comprise: Aerococcus, Aloiococcus, 
Carnobacterium, Enterococcus, Lactobacillus, Lactococcus, Leuconostoc, Oenococcus, Pediococcus, Streptococcus, Tetragenococcus, Vagococcus and Weisella, among others (4).

Due to its historical utilization in food industry, they have been classified as "Generally Regarded as Safe” (GRAS) by the Food and Drug Administration of the United States of America (FDA) and they have also been designated with the "Qualified Presumption of Safety" (QPS) according to the European Food Safety Authority (EFSA). Consequently, in the last decades increasing interest and lines of investigations have been opened concerning other fields and applications of LAB, which is how from food preservation and starter cultures they expanded into probiotics development, enzymes production, drugs delivery, vaccines formulation, among others.

Nevertheless, within the LAB group there are also some controversial species, which are pathogens or opportunistic pathogens, like members of the Streptococcus and Enterococcus group (Fig. 1). Even though some strains of Enterococcus are able to produce bactericidal compounds and therefore their biotechnological applications are encouraged, special care need to be taken when working with microorganisms prone to acquire antibiotic resistances and turn into nosocomial pathogens (see below).

Members of LAB, apart from the acidifying effect on growth media as previously mentioned, are also efficient inhibitors of undesired microorganisms due to the synthesis of bacteriocins. These are peptidic compounds with bactericidal effects on closely related bacteria from the same ecological niche, which provides an evolutionary advantage to the producer strain (5). Its effects have also been proven in more distant species, mainly when environmental conditions can be adjusted to favor the bacteriocin mode of action. The first recognized bacteriocin synthesized by L. lactis was nisin, which has been employed for decades in food industry for its antimicrobial action against members of Gram-positive bacteria. Noteworthy, it also inhibits growth of Clostridium and Bacillus spores, and different combinations of nisins, nisin producer starter strains and other preservation mechanisms have been applied in food industry and fermentation processes without health risks for consumers $(6,7)$. 


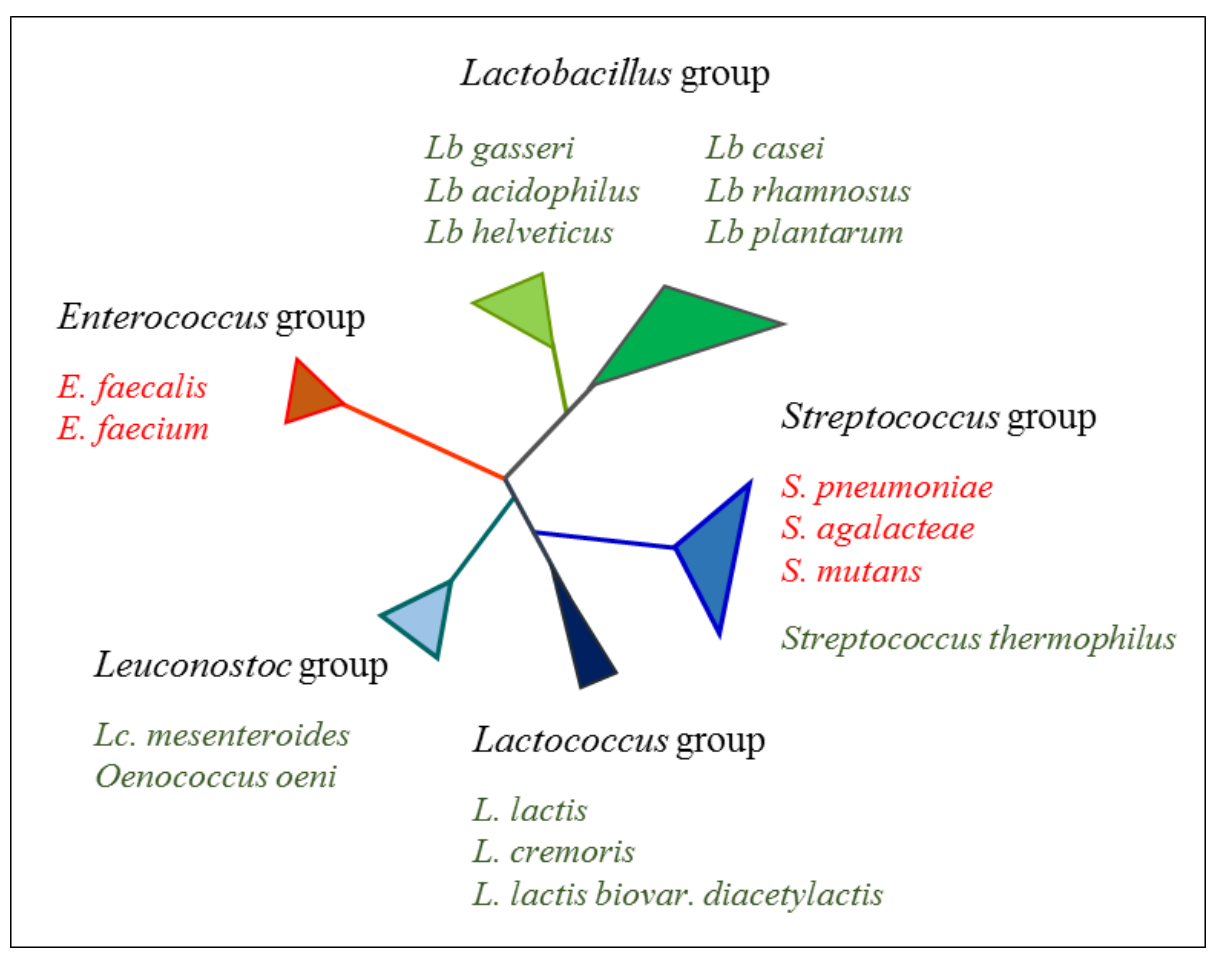

Fig. 1 Schematic phylogenetic tree of LAB based on 16 rRNA gene sequence homology. Representative LAB species related to the food industry are included. Species that do not represent a risk for human health are depicted in green, opportunistic pathogens in red.

Other bacteriocins active against pathogenic bacteria in human infections have prompt the development of probiotics. Probiotics are live microorganisms that, when administered in adequate amounts, confer a health benefit on the host (8). The intestinal lumen is an elaborated matrix with complex microbiota that varies for each host and is influenced by different direct and indirect agents. Examples of indirect agents are the use of antibiotics and also the diet, which has been referred to as the most efficient way of modulating gut microbiota (9) . Diets complemented with probiotics and symbiotics have a beneficial impact on the immune system of individuals with infectious bacterial diseases and even a connection was established between the increase of Lactobacillus levels and the vitamin A supplementation for Norovirus treatment (10). Interesting results have been obtained as well for Helicobacter pylori inhibition by use of LAB isolated from fermented noodle (11), and bacteriocins synthesized by LAB have also been reported to be active against pathogenic bacteria like Listeria monocytogenes, Staphylococcus aureus and Streptococcus mutans $(12,13)$. 


\subsubsection{Lactococcus lactis}

Lactoccus lactis is one of the most used starter culture in the cheese making industry as well as the best characterized LAB (14). Several strains have been thoroughly studied for their impact on the fermented final product, and they fall mainly into two subspecies, L. lactis subsp. cremoris (preferentially used as starter since they cause less bitterness), and L. lactis subsp. lactis (known as a fast acidifier). A biovariety can also be found within L. lactis species, i. e. $L$. lactis subsp. lactis biovar. diacetylactis, capable of citrate fermentation and therefore affecting flavor development via acetoin and diacetyl synthesis (15).

From an evolutionary point of view, L. lactis has been able to colonize different ecological niche, like plant surfaces, the urogenital and gastrointestinal tract of mammals as well as other animal tissue surfaces. It can also be found in different types of foods including dairies, meats and fermented products. Strains from diverse sources have evolve loosing or gaining specific sets of genes which made possible their adaptation to the defined media employed in industries, the rich media of dairy matrices and the most demanding natural environments of non-dairy sources such as plants. Consistent with this, a recent study performed on 30 sequenced genomes available on the NCBI database belonging to L. lactis taxon, reported that $16 \%$ of the unique gene families of each genome correspond to phage proteins acquired through the integration of a particular pro-phage element (16). Moreover, about $19 \%$ of these gene families showed to be involved in mobilization and conjugation, transposases and IS elements, or genes encoding systems providing a beneficial niche specific advantage related to bacteriocin production, sugar metabolism and restriction-modification systems. This study also showed a clear division between lactis and cremoris subspecies, and allowed the determination of the core genome sizes being of 1406 and 1413 genes, respectively (plasmids were excluded from the study) (16). For industrially relevant $L$. lactis strains, the most important set of genes are those coding for proteins that enable lactose fermentation, the prevalent carbon source in dairy products. These genes are encoded in the lacABCDEFGX operon, which is found in plasmids of all cremoris subspecies, except for the plasmid free strain MG1363 and its derivative NZ9000. On the other hand, within lactis subspecies the nondegrading lactose strains are more represented, being IL1403 one of these. Altogether, L. lactis is a very versatile species with a wide research history and range of options for industrial applications, which are currently under investigation. 
L. lactis subsp. lactis IL1403 (from now on L. lactis IL1403) was the first sequenced lactis strain, and it is the plasmid free version of strain IL594 isolated from a cheese starter culture (17). Together with strain NZ9000 and MG1363, it is the most used genetic and physiological L. lactis model in the laboratory (14) (for a summary of L. lactis strains used for research and industrial applications see Fig. 2 and Table I). L. lactis IL1403 has been employed in research for decades and its consequent adaptation and domestication led to a small genome of approximately 2500 open reading frames (ORF), nearly the half of other well-known bacterial models such as Bacillus subtilis. As a member of LAB, this strain is expected to be strictly fermentative, but genes related to aerobic respiration have been found in L. lactis IL1403 genome (18). Namely, men and $c y t A B C D$ operons are present, involved in menaquinone synthesis and cytochrome d biogenesis respectively, and even hemH, hemK and hemN for late steps of heme biosynthesis (oxidation of porphyrinogen and attachment of iron to heme), although no genes for the early steps were identified.

L. lactis ssp. cremoris MG1363 was initially obtained as the plasmid cured progeny of the dairy origin NCD0712 strain (19) and it is today the model laboratory strain for genetic engineering and biotechnology of L. lactis (Fig. 2). From this strain also derives one fundamental tool for the nisin-controlled gene expression system (NICE), which was obtained via replacement of pepN gene (coding for an aminopeptidase) for the nis $K$ and nisR genes (20), encoding a histidine-kinase and a cytoplasmic response regulator, respectively. The strain thus created, named NZ9000, is able to sense nisin in the medium upon which NisK autophosphorylates and transfers the phosphate to the counterpart of the two-component system, NisR, being thus activated. The active version of NisR induces in turn the PnisA promoter, present in the pNICE series of vectors, for which expression of a gene of interest cloned under it is successfully induced (21). It is important to mention that L. lactis NZ9000 lacks 1821 nucleotides in the pepN-napC locus and when compared to the original strain MG1363, it has 6 point mutations independently acquired, being otherwise genetically equivalent (22).

Another tool developed for $L$. lactis engineering and biotechnology, of special relevance for this work, is strain clpP-htrA, which shows reduced proteolytic activity, due to the lack of both major proteases Clp and HtrA (Fig. 2). Consequently it is a good strategy for the stable production of a heterologous protein of interest (23). 


\section{L. lactis ssp. lactis IL594 \\ L. lactis ssp. lactis IL1403 \\ pIL 1 -7 \\ Plasmid free}

L. lactis ssp. cremoris NCD0712

pLP712 1-5

pNZ712

$\checkmark$

L. lactis ssp. cremoris MG1363

Plamid free
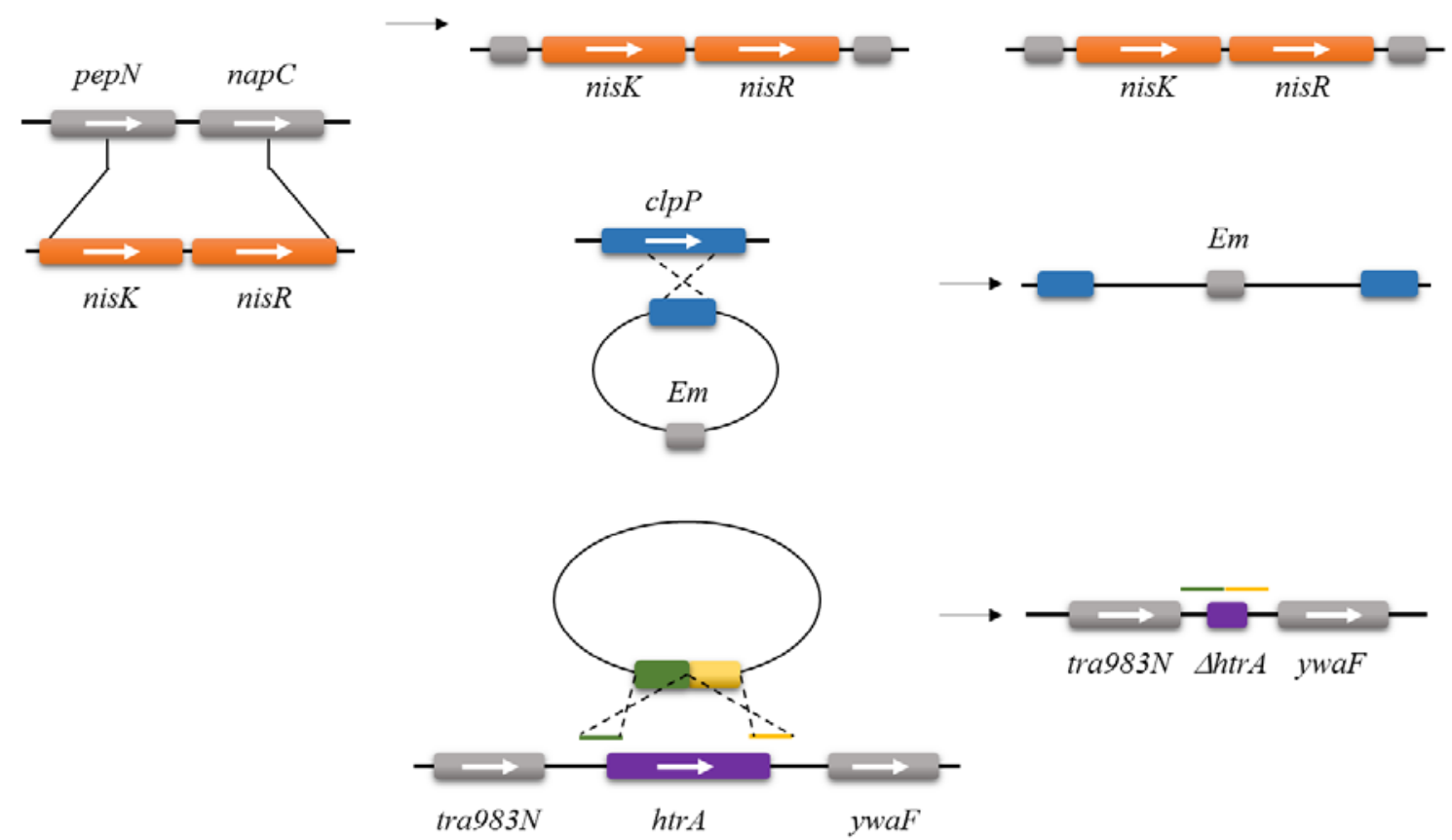

Fig. 2 Representative diagram of the development of $L$. lactis strains commonly used for genetic engineering and gene expression. L. lactis IL594 contains seven plasmids (pIL1 to 7), and is the parental strain of the plasmid free IL1403 strain. On the other hand, L. lactis MG1363 is the free plasmid version of strain NCDO712. It is also the parental strain of NZ9000, which carries the nisKR insertion, for nisin sensing and activation of the NICE system vector series. Strain clpP-htrA lacks the proteolytic enzymes HtrA and ClpP due to a deletion in the former case, and an erythromycin gene replacement, in the latter.

\subsubsection{Enterococcus faecalis}

Enterococcus faecalis is a natural commensal member of the human gut flora, and a very versatile bacterium for diverse niches colonization. As a member of the Enterococcus genus, it can grow in temperatures ranging from $10^{\circ} \mathrm{C}$ to $45^{\circ} \mathrm{C}$, it can survive acidic and alkaline conditions (from $\mathrm{pH} 4.0$ to 9.6), and can resist high salt concentrations (up to 6.5\% $\mathrm{NaCl}$ ) (24). E. faecalis can be found in plant materials and foods, especially in fermented products of animal origin, although in water and non-fermented meat articles its presence is not desired and normally it is used as an indicator of sanitary quality (25). 
E. faecalis is as well one of the most controversial LAB, since it can participate in DNA exchange, being able to acquire antibiotic resistances, genes related to haemolysin-cytolysin production, proteins involved in adhesion to host tissue, or other virulence factors (26). The most complicated strains are the vancomycin resistant, which normally enter the host via wounds and catheters, causing endocarditis, bacteraemia and urinary tract infections (27). Nevertheless, due to E. faecalis wide genetic variability, strains isolated from clinical samples are significantly different from those isolated from food. Abriouel et al. showed that species most frequently involved in human diseases are also most frequently associated with clinical samples and in the same study enterococcal isolates from fruits and vegetables showed a much lower incidence of antibiotic resistance compared to clinical samples (28).

Notwithstanding its controversial profile, it is a reality that Enterococcus (mainly E. faecalis, E. faecium and E. durans) are part of food products, either due to contamination along the manufacturing process or as part of dairy starter, adjunct and/or non-starter cultures (29). Moreover, E. faecalis is the species with the most acidifying, proteolytic and lipolytic activities, which as previously mentioned, exerts a strong contribution to flavor development. It is well known nowadays that its presence in food products like cheese and fermented meats contributes to the ripening process in the development of favorable organoleptic features (30).

Some strains of $E$. faecalis have even been reported as probiotics and others are known for its bacteriocin production (31). E. faecalis synthesizes enterocin AS-48, which has a broad inhibitory spectrum, and offers interesting possibilities for food preservation. It has been shown to be active against Gram-positive bacteria like L. monocytogenes, S. aureus, Mycobacterium, Bacillus cereus and even some Gram-negative bacteria (32-34).

\subsection{Potassium homeostasis}

Ion homeostasis is a key factor for life in the three kingdoms. Particularly, potassium $\left(\mathrm{K}^{+}\right)$is the most abundant cation in the cytosol, and its uptake is essential and tightly regulated in all living cells. It intervenes in important cellular processes like translation, being essential for prokaryotic 50S and 30S ribosomal subunits activity, in charge of the peptidyl transferase reaction and the binding of phenylalanine-transfer RNA in vitro, respectively (35, 36). Potassium concentration is a limiting factor for protein synthesis, and it has been reported that the rate of protein synthesis slows down fourfold when $\mathrm{K}^{+}$concentrations are under $25 \mathrm{mM}$ (37). 
Many enzymes require potassium as cofactor and it is also linked to bacterial bioenergetics, since it affects both components of the proton motive force (PMF), the $\mathrm{pH}$ gradient $(\Delta \mathrm{pH}$, alkaline inside the cell) and the electric potential across the cellular membrane ( $\Delta \psi$, negative inside the cell) (38). Secondary transporters involved in $\Delta \mathrm{pH}$ generation, generally use the PMF generated by respiration or ATPases to actively uptake protons in exchange for cytoplasmic cations like $\mathrm{Na}^{+}$or $\mathrm{K}^{+}$(39). Therefore, potassium metabolism also affects $\mathrm{pH}$ homeostasis via cation-proton antiporters, especially crucial for non-respiring LAB, which acidify growth media and need to maintain alkaline values of $\mathrm{pH}$ inside the cells when extracellular values can go down to lower limits than their growing capacity (40).

The $\Delta \psi$ component of the PMF is also related to potassium metabolism since in alkaline $\mathrm{pH}$ homeostasis, it enables the electrogenic influx of protons in exchange of an unequal ratio of $\mathrm{Na}^{+}$or $\mathrm{K}^{+}$efflux. Nevertheless, $\mathrm{Na}^{+} / \mathrm{H}^{+}$antiporters are more often involved in this process, whereas $\mathrm{K}^{+} / \mathrm{H}^{+}$antiporters take relevance under $\mathrm{Na}^{+}$limitation $(39,41)$. In turn, this membrane potential is also important for ATP production and nutrient uptake, for which potassium metabolism influences cell growth as well.

Cells also need a mechanism to alter their intracellular $\mathrm{K}^{+}$concentrations in response to external solute changes. In these respect, potassium channels play a crucial role in osmotic stress responses. Most bacteria growing at low osmolarity use $\mathrm{K}^{+}$as primary osmotic solute and when grown in high osmolarity media, potassium acts as a second messenger to trigger accumulation of compatible solutes. (42). In this sense bacterial cell turgor and cell viability under variable environmental conditions are maintained broadening colonization possibilities.

It is important to mention that osmoregulation is also affected by the pool of compatible solutes. These molecules help reaching homeostasis after osmotic changes in the environment. Water efflux when osmolality increases outside the cell is first coped with potassium intake. Nevertheless, this is unsustainable when prolonged in time since it can disturb protein synthesis and other cellular processes and interactions affected by $\mathrm{K}^{+}$. Bacteria have therefore evolved developing a second phase of adaptation to osmotic stress, by synthesis of osmoprotectant solutes like betains, polyols, sugars, amino acids and compounds derived from them (43). A complex matrix is therefore established around potassium metabolism, connecting different cellular pathways in order to maintain homeostasis. 


\subsubsection{Potassium uptake systems}

Bacteria have evolved different systems to accumulate potassium intracellularly and achieve stable concentrations in order to cope with basic metabolic demands (40). Different high and low affinity uptake mechanisms are involved, if the bacteria are growing in media with low or high $\mathrm{K}^{+}$concentrations, respectively.

In E. coli, intracellular concentrations of $\mathrm{K}^{+}$are kept close to $200 \mathrm{mM}$, when concentration in growth media is $10 \mathrm{mM}$ (31). In this bacterium, there are three main potassium transport systems: Trk, Kdp, and Kup. Together they ensure potassium uptake in different growth conditions, and although mutants in the three systems cannot grow in regular media (unless at least $115 \mathrm{mM} \mathrm{K}^{+}$is added), one of them is already enough for growth $(44,45)$.

Trk system consists of four genes, which are constitutively expressed, with trkA as the main component and its product the predominant $\mathrm{K}^{+}$transporter at neutral $\mathrm{pH}$. It has low affinity for the ion and depends on ATP and proton motive force for potassium uptake (44). On the other hand, the KdpATPase system includes the inducible high affinity transporter KdpA, which is synthesized at low $\mathrm{K}^{+}$concentrations and under osmotic stress (46). Finally, Kup (formerly TrkD) is also a constitutive but low affinity system and its activity increases at low $\mathrm{pH}$, when TrkA and Kdp activities are insufficient (47).

In other model bacterium like B. subtilis, S. aureus and L. monocytogenes, one of the main systems in charge of potassium uptake is Ktr, which like Trk belong to the Trk/Ktr/HKT family (48). Moreover, two different affinity systems can be distinguished within constitutive Ktr transporters: KtrCD is a low affinity system present in the three bacteria mentioned, whereas high affinity KtrAB is also present in B. subtilis, where it is regulated by the kimA riboswitch. Interestingly, another copy of the riboswitch can be found in B. subtilis, regulating as well $\operatorname{kimA}$ (formerly $y d a O$ ), coding for a member of a recently discovered potassium transporter family (49). An increase of $\mathrm{K}^{+}$concentration results in accumulation of c-di-AMP which binds to the riboswitch and consequently represses expression of the downstream encoded transporters (49). Interestingly, the second messenger c-di-AMP (described in the following section) was proved to bind subunits KtrA and KtrC as well, upon which inhibition of the respective transporters occurs (50).

As previously mentioned, potassium metabolism is an intricate network affecting diverse pathways, and so is potassium uptake. Even though a brief outline is made here as 
regards major $\mathrm{K}^{+}$transporters in model bacteria, it cannot be ignored that other minor systems exist as well.

\section{3. (3',5') cyclic-di-adenosine-monophosphate}

(3’,5’) cyclic-di-adenosine-monophosphate (c-di-AMP) is a second messenger recently discovered in the crystallographic structure of DisA, a protein in charge of scanning DNA integrity in Thermotoga maritima (51). Therefore, the first function assigned to this compound was related to the DNA-damage dependent cell cycle control. Later it was also linked to cellular growth and cell wall homeostasis (52) as well as sporulation (53) and other cellular pathways like antibiotic resistance and nitrogen metabolism, among others (54).

In pathogenic organisms, it was reported that c-di-AMP has an important role in the establishment of infection. For instance, in L. monocytogenes, c-di-AMP secretion after infection induces the immune response mediated by interferon $\beta$ (55) and in Streptococcus pneumoniae it was observed that a double mutant strain lacking c-di-AMP degrading enzymes GdpP and PgpH (described below) is no longer capable of infection. Moreover, both proteins are involved in four key stages of pneumococcal pathogenesis: colonization, otitis media, pneumonia and bacteremia (56).

c-di-AMP homeostasis is tightly controlled; low concentrations as well as its accumulation are detrimental for the cell. In B. subtilis, high levels of c-di-AMP lead to mutations that inactivate the activity or expression of its synthesizing enzyme, CdaA (see below) (54), and in L. monocytogenes, a double mutant in GdpP and PgpH results in growth defects inside and outside the host (57). Moreover, S. aureus strains lacking GdpP increase intracellular concentrations of c-di-AMP and are more resistant to acid stress and in general, higher concentrations of this second messenger are associated to increased resistance to $\beta$ lactam antibiotics (52, 58, 59).

The main interest on this compound was related to its essential role in rich media for low GC Gram-positive bacteria, since only in 2015 it was possible to obtain a mutant strain for the only c-di-AMP synthesizing enzyme in L. monocytogenes, in strictly controlled minimal media (60).

In the last years, investigations were centered mainly in the role of c-di-AMP in potassium homeostasis and osmolyte transport, and even though its interaction to potassium 
transport components was previously proven $(50,61)$, it was only recently that a direct connection was made between $\mathrm{K}^{+}$metabolism and c-di-AMP essential role (49). In B. subtilis, it was shown that this second messenger also regulates synthesis of $\operatorname{KimA}\left(\mathrm{K}^{+}\right.$importer $\left.\mathrm{A}\right)$ via the $\operatorname{kim} A$ riboswitch, a copy of which is also found upstream $k \operatorname{tr} A$, regulating its expression as well. Moreover, in the same study, a B. subtilis strain lacking c-di-AMP synthesizing enzymes was obtained under extremely low potassium concentrations (49) and in S. aureus this was possible in chemically defined medium and in rich medium supplemented with sodium or potassium chloride (62).

\subsubsection{Synthesis of c-di-AMP}

c-di-AMP is synthesized by specific di-adenilate cyclases from two ATP molecules, with the exception of $M$. tuberculosis, which can also produce it from two molecules of ADP (63). Three types of DAC containing enzymes are so far described, and they are all present in the model organism B. subtilis. The cyclase enzyme DisA, can be found in actinobacteria and in spore-forming firmicutes, and it associates into two interacting tetramers. It is involved in DNA integrity control and repair, being inhibited by damaged DNA as well as atypical DNA arrangements such as Holliday junctions (51). On the other hand, CdaS cyclase is only found in spore forming Bacillus members and in one Clostridium species, and it is exclusively expressed during sporulation, guaranteeing spores efficient germination. It contains two $\alpha-$ helices at the N-terminal end, followed by the DAC domain at the C-terminus (64) The third cdi-AMP synthesizing enzyme, CdaA, is the most frequently found in firmicutes. Similarly to CdaS, the DAC domain is located at the C-terminal end, whereas three $\alpha$-helices can be found at the N-terminus (Fig. 3). It is important to mention that DAC containing proteins are extensively distributed in both Gram + and - bacteria, being much frequently found in the latter, and normally associated to additional domains in charge of the input and output of a variety of signals. Nevertheless, in the vast majority of low GC firmicutes, only one protein with a DAC domain is typically found.

In many $\delta$-proteobacteria and firmicutes, $c d a A$ is encoded in a widely conserved gene cluster (described for L. lactis in section 3.1.6., Fig 42), which comprises $c d a R$, codifying for a CdaA regulatory protein, and $\operatorname{glm} M$, coding for the phosphoglucosamine-6-phosphate mutase enzyme, capable of converting $\alpha$-D-glucosamine-6-phosphate into D-glucosamine-1phosphate, a cell wall precursor. It is therefore not surprising that one of the first pathways 
associated to c-di-AMP was cell wall biosynthesis, since genes responsible for the synthesis of related proteins are normally clustered together in the DNA.

As previously mentioned, c-di-AMP is essential when bacteria are grown in rich media. Consequently, many efforts are being directed into the development of drugs able to inhibit cdi-AMP cyclase activity and being therefore suitable for antibiotic formulation. For example, the anti-parasitic urea-derived drug suramin (used for African trypanosomiasis and onchocerciasis treatment) is proven to inhibit T. maritima DisA, competing with ATP for the same binding site in the enzyme (65).

Altogether, c-di-AMP synthesis and metabolism are involved in diverse cellular pathways, and even though an increasing amount of information as regards this second messenger is being generated nowadays, its potential applications continue to expand.

\subsubsection{Degradation of c-di-AMP}

Bacteria capable of c-di-AMP synthesis also contain specific hydrolyzing enzymes in charge of its degradation. These proteins are phosphodiesterases, and can be divided into two families. The first family comprises homologues of a membrane-bound protein named GdpP, which consists of two transmembrane helices, a degenerate PAS domain (Per-Arnt-Sim, for signal transduction, (66), a modified GGDEF domain and a DHH-DHHA1 domain with a catalytic motif Asp-His-His. On the other hand, the second family includes an HD domain, where a His-Asp motif can be found, and it is separated of an N-terminal extracellular domain by seven transmembrane helices (Fig. 3) (67). Homologues of this group are distributed in cyanobacteria, bacteroidetes, fusobacteria, species of the genus Thermotoga and members of firmicutes, where proteins of both families are normally found together.

The most studied phosphodiesterase, GdpP, is present in firmicutes, actinobacteria and spirochaetes. It degrades c-di-AMP into the dinucleotide 5'pApA and belongs to the DHHDHHA1 family along with another kind of phosphodiesterase, found for instance in $S$. pneumoniae, presenting a unique soluble DHH/DHHA1 domain (68). This enzyme is capable of degrading 5'pApA into two molecules of AMP and it is a B. subtilis NrnA homologue, although in this microorganism it does not hydrolyses c-di-AMP. This single domain version is of course smaller and cytoplasmic, and it is present in Borrelia burgdorferi y M. tuberculosis as the only c-di-AMP degrading phosphodiesterase $(69,70)$. 
Phosphodiesterases from both groups have been proved to be controlled by the signaling nucleotide guanosine-tetraphosphate (ppGpp), establishing a link between c-di-AMP metabolism and the stringent response, designed to conserve energy during nutrient starvation. Both GdpP and PgpH (HD family) have been proved to be strongly inhibited by ppGpp (71, 72)

Biochemical characterizations have shown that phosphodiesterases have multiple functions and regulatory inputs, including ppGpp, heme group and nitric oxide union and ATPase activity (73). Moreover, gdpP mutants of $S$. aureus and B. subtilis were significantly more resistant to $\beta$-lactamic antibiotics and in general, for mutations in this enzyme phenotypes of stress resistance to $\mathrm{pH}$, high temperatures and compounds affecting cell wall stability were obtained $(58,74,75)$.

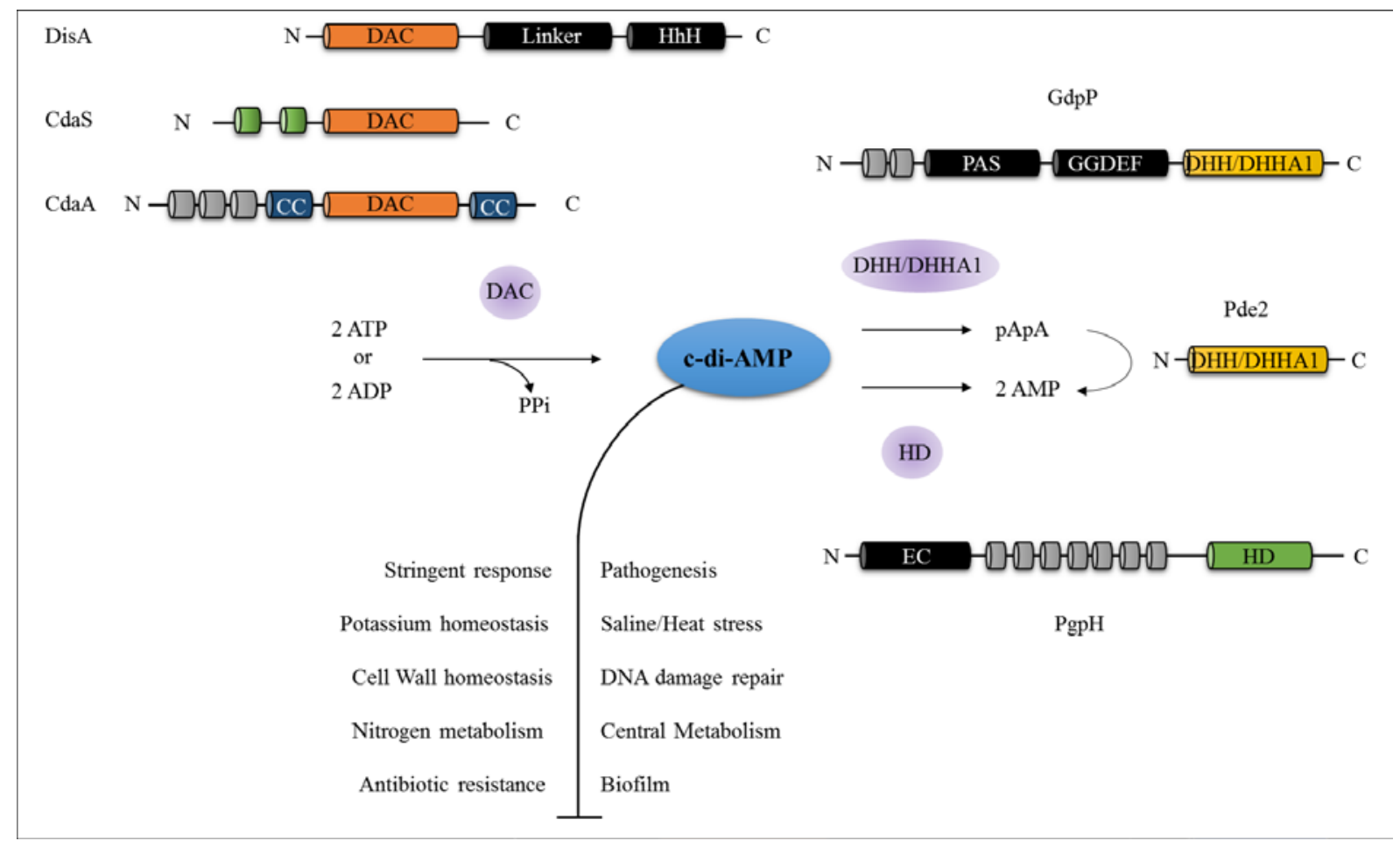

Fig. 3. Representation of c-di-AMP synthesis and degradation. DAC containing enzymes (orange) are in charge of c-di-AMP synthesis. The three types of cyclases are represented: DisA, CdaA and CdaS. c-di-AMP is synthesized from two molecules of ATP, although M. tuberculosis and S. mutans can also use two molecules of ADP as substrate. The two phosphodiesterases families are depicted on the right: HD (green), hydrolyzing c-diAMP to AMP and DHH/DHHA1 (yellow). Within this family two types of enzymes can be distinguished: the most frequently membrane-bound version, which yields pApA, and the smaller soluble version, which can further degrade c-di-AMP to AMP. Principal pathways related to c-di-AMP metabolism are also indicated. 


\subsection{3. c-di-AMP interaction partners}

The first reported c-di-AMP interaction protein was DarR of Mycobacterium smegmatis. This protein is a transcriptional factor of the TetR family, which is stimulated by c-di-AMP and consequently binds to the promoter region of its own gene, repressing its expression. It also represses expression of a divergent operon, involved in fatty acid metabolism and of $\operatorname{csp} A$ gene, encoding a cold shock protein (76).

As previously described, this second messenger has a strong influence in potassium homeostasis and its interaction to different potassium transporters has been reported. c-di-AMP binds to KtrA, the cytoplasmic regulatory component of the KtrAB potassium uptake system. The binding site is the regulator of conductance of $\mathrm{K}^{+}$(RCK_C) domain, oriented towards the cytosol, and upon binding, it inhibits potassium uptake in B. subtilis, S. aureus and $S$. pneumoniae $(50,77)$. Interestingly, c-di-AMP in B. subtilis also binds to the kimA riboswitch, which regulates expression of $k \operatorname{tr} A$ as well as $k i m A$, coding for a recently reported potassium importer (49). Moreover, in S. aureus this second messenger regulates all potassium uptake systems so far described, which includes KtrC and the KdpD subunit of the KdpDE two component system previously described $(50,61)$.

Noteworthy, the RCK domain is present in a wide variety of proteins, like CpaA of $S$. aureus, a putative cation/proton antiporter recently proven to be regulated by c-di-AMP as well. Contrary to the c-di-AMP binding proteins so far described, this second messenger activates CpaA in S. aureus (78). On the other hand, PstA, a PII like signal transduction protein, present in S. aureus, L. monocytogenes and B. subtilis (where it is named DarA) also binds c-di-AMP, and even though several investigations were carried to elucidate its interaction to c-di-AMP, its function remains unknown $(50,54,79)$.

As stated, osmoregulation involves not only potassium homeostasis but also regulation of the synthesis and uptake of other osmolytes. c-di-AMP has been related to osmolarity homeostasis in both ways, i.e. potassium intake and osmoprotectants metabolism. For instance, c-di-AMP also interacts with OpuCA, the ATPase component of the carnitine ATP-binding cassette transporter OpuC (80). Interestingly, binding of c-di-AMP to OpuC occurs in the cystathionine- $\beta$-synthase (CBS) domain, broadening the possibilities of interactions to CBScontaining, like L. monocytogenes CbpA and CbpB proteins of unknown function (81). 
In L. monocytogenes, c-di-AMP also binds to CabP, a B. subtilis KtrA homologue, and in this bacterium as well as in L. lactis, this second messenger was linked to central metabolism, since it allosterically regulates pyruvate carboxylase activity (81). c-di-AMP connection to central metabolism and the enzymes encoded in the $c d a A R$ glmM operon are more detailed described for LAB (see below).

\subsection{4. c-di-AMP and LAB}

Even though LAB importance in basic and applied science is undeniable, only a few approaches with regard to c-di-AMP metabolism were done so far. The synthesizing enzyme CdaA is encoded in the widely conserved $c d a A R$ glmM gene cluster, in which the gene for the regulatory protein CdaR is also present. The third gene of the operon codes for GlmM, an enzyme which converts glucosamine-6-phosphate to the peptidoglycan precursor glucosamine1-phosphate, as previously described.

Different combinations of L. lactis cdaA operon genes expressed in E. coli, confirmed that CdaR and CdaA also interact in this bacterium and evidence suggests that CdaR would negatively regulate CdaA (82). Moreover, GlmM also modulates CdaA activity, connecting cdi-AMP metabolism to cell wall biosynthesis in L. lactis as well. Nevertheless, the exact mechanism remains unknown and, based on other model organisms, it is hypothesized that it would be through protein-protein interaction (82).

One of the first studies carried out in L. lactis, allowed the identification of spontaneous $g d p P$ mutants after a high temperature incubation step. Among these mutants, heat-resistant and salt-hypersensitive phenotypes were observed, as well as enhanced growth in sub-lethal concentrations of penicillin G (74). Moreover, mutants in which GdpP activity is diminished manifest greater acid stress resistance, and this protein was also linked to the stringent response, since ppGpp exerts a strong inhibition on the c-di-AMP hydrolyzing activity of the DHH/DHHA1 domain (72, 83). Furthermore, it has been proposed that PAS domains act as heme sensors, since upon binding of this compound, GdpP is strongly inhibited (84). This would protect cells from heme toxicity, and in accordance to this, L. lactis gdpP mutant manifested increased sensitivity to heme group compared to the wild type (85).

On the other hand, it has been recently proved that pyruvate carboxylase (PC) enzyme from E. faecalis binds and is regulated by c-di-AMP. Interestingly, the binding site does not 
seem to be conserved since in silico studies show that it is different from the c-di-AMP binding site of L. monocytogenes PC (86). This study was also extended to L. lactis and it was confirmed that c-di-AMP also binds to PC in this bacterium, being thus inhibited. Due to the lack of an $\alpha$ ketoglutarate and glutamate dehydrogenase enzymes, and the presence of a non-functional isocitrate dehydrogenase, the TCA cycle is truncated in L. lactis. Consequently, it is proposed that the main function of PC is the de novo synthesis of aspartate via AspC. Very importantly, aspartate is a precursor for other amino acids, for pyrimidine and also for peptidoglycan crossbridge amino acids. It is also theorized that PC is indirectly involved in osmoprotectant compounds synthesis, which is also one of the homeostasis mechanisms in which c-di-AMP is involved and would account for c-di-AMP interaction to a central metabolism enzyme (86).

c-di-AMP synthesizing and degrading activities were confirm in CdaA and GdpP homologous proteins of other LAB, like Streptococcus suis and Streptococcus pyogenes (8789). RT-PCR studies showed in the last case that $g d p P$ gene is expressed at least with two downstream genes, rpl9 and holB. The former codes for the ribosomal protein L9, whereas the latter encodes the $\delta$ ' subunit of DNA polymerase III (90). In the same work, a gdpP mutants study linked the lack of GdpP with increased resistance to the $\beta$-lactam antibiotic ampicillin, and the location of this enzyme inside the cell to the biosynthesis of the streptococcal virulence factor SpeB (secreted pyrogenic exotoxin B). Moreover, a $\Delta g d p P$ strain showed attenuated virulence in a murine model of subcutaneous infection.

In S. pneumoniae one CdaA homolog was also confirmed and as previously mentioned, two phosphodiesterases were identified, named Pde1 and Pde2. Pde1 is the GdpP homolog, whereas Pde2 is the shorter cytoplasmatic version described above (Fig. 3), and although both can degrade c-di-AMP, the cleavage products resulting from each are different: Pde1 cleaves c-di-AMP into pApA and Pde2 directly hydrolyzes it to AMP. Furthermore, Pde1 as well as Pde2 were shown to contribute to pneumococcal virulence, and even though Pde2 orthologs can be found in B. subtilis, S. aureus and L. monocytogenes, degradation of c-di-AMP by these enzymes has not been reported yet $(56,68)$.

Studies looking for c-di-AMP interaction partners in $S$. pneumoniae led to the identification of CabP, which belongs to the Trk/Ktr/HKT family of proteins and is the peripheral regulatory subunit of the potassium transporter SPD_0076. c-di-AMP binding to CabP would alter its conformation, disrupting the interaction between both parts of the $\mathrm{K}^{+}$ 
uptake system, being thus inhibited (77). Interestingly, CabP interaction to c-di-AMP was also used to develop a c-di-AMP quantification method via ELISA (91).

In S. mutans a CdaA homologue was also identified, being able to synthesize c-di-AMP from ATP as well as ADP. Interestingly, a cdaA mutant was obtained in the same study, showing increased susceptibility to peptidoglycan-targeting antibiotics and oxidative stress, and also higher autolytic activity than the wild type strain (92, 93). In a different study it was also proved that c-di-AMP mediates biofilm formation of S. mutans, and high levels of the second messenger up-regulates expression of $g t f B$, an enzyme in charge of water-insoluble glucans production, critical for biofilm formation and virulence of this bacterium (94).

In the case of pathogenic bacteria, like the opportunistic LAB Group B Streptococcus (GBS), c-di-AMP can be secreted, activating consequently the Interferon- $\beta$ (IFN- $\beta$ ) response of the host. Interestingly, S. agalactiae, belonging to GBS, keeps extracellular c-di-AMP levels low thanks to CdnP, an ectonucleotidase anchored to the cell wall. It is important to mention that c-di-AMP degradation is different from those previously described for phosphodiesterases, since CdnP acts sequentially with another ectonucleotidase, NudP, to degrade c-di-AMP into adenosine (95). In this way, over-activation of STING and its concomitant IFN- $\beta$ induction is avoided and virulence is promoted.

Finally, in the opportunistic pathogen E. faecalis previously introduced, both classes of phosphodiesterases GdpP and PgpH where found encoded in its genome. Nevertheless, the obtaining of mutants involved in daptomycin resistance (an antibiotic targeting the cell membrane) only resulted in $g d p P$ mutants, in agreement with previous research stating that this protein is more important for virulence than $\mathrm{PgpH}(57,96)$. Initial mutations were also obtained in the liaFSR pathway, a three-component membrane stress response regulator, and it was observed that deletion of liaR lead to an increase in c-di-AMP intracellular levels. Even though the interaction between LiaFSR and c-di-AMP is not fully understood, the link between cellular wall damage and c-di-AMP levels was again established (96).

\subsection{American Trypanosomiasis (Chagas disease)}

American Trypanosomiasis (also known as Chagas disease) is a tropical disease caused by the flagellate protozoan parasite Trypanosoma cruzi. Even though the first cases were reported in the tropical areas of Latin America, according to the Health World Organization, an 
estimate of 8 million people are infected worldwide with a cost of 10.000 deaths per year, affecting nowadays countries of North America, Europe, Asia and Australia as well (Fig. 4).

The life cycle of $T$. cruzi consists of four stages: the replicative epimastigote and amastigote stages and the infective non-replicative metacyclic and trypomastigote stages. It also requires a host of the Triatomine subfamily, most commonly known as kissing bugs, or Vinchucas in Spanish. When these bugs bite a mammal in order to feed from its blood, they normally defecate nearby and the metacyclic trypomastigotes residing in the faeces (which normally cannot penetrate the skin) can now enter the new host through the wound. After infection, they transform into amastigotes so they replicate inside the host cell and differentiate into trypomastigotes. Once the host cell disrupts, the trypomastigotes are released to the blood stream and are ready to infect new cells (97). There are also other less frequent routes of infection, such as blood transfusion, organ transplant and vertical transmission, i.e. 2-10\% of infants are infected by their infected mothers. Horizontal transmission is possible as well, but more rare, since it implies mucosal penetration through the mouse or eyes (98).

Chagas disease manifests two clinical phases: the short acute phase of patent parasitemia, which is oligosymptomatic, and the chronic phase, which can remain asymptomatic for months or even years. The main symptoms in the chronic stage are related to heart failures and pathologies affecting organs of the gastrointestinal tract. For more than 40 years, Chagas disease treatment has been based on the nitroheterocyclic compounds nifurtimox (NFX; 3-methyl-4-[59-nitrofurfurylideneamine] tetrahydro-4H-1,4-tiazine-1,1-dioxide; developed by Bayer, Germany) and benznidazole (BZ; N-benzyl-2-nitroimidazole acetamide; RO7-1051; developed by Laboratorio Farmacéutico do Estado de Pernambuco (LAFEPE), Recife, Brazil and Laboratorio ELEA, Ciudad Autónoma de Buenos Aires, Argentina), which have trypanocidal activity against all parasitic forms. The main drawbacks of these drugs are: 1) their mechanisms of action are not entirely clear, 2) their side effects, which normally are the cause of treatment interruption and 3) the low efficacy, since during the acute phase only 50 to $80 \%$ of patients are cured and when used in the chronic phase, percentages drop to $8-20 \%$ $(99,100)$.

The World Health Organization (WHO) has established a set of characteristics that the ideal drug for Chagas disease treatment should gathered, namely: parasitological cure in both the acute and chronic phases, efficacy in one dose or a few doses, accessibility to affected population (low cost), absence of side effects or teratogenic effects, and no induction of 
resistance. Unfortunately, there is no drug meeting all these requirements, and consequently new compounds are currently under investigation. Nonetheless, apart from a cure, priorities also fall into the improvement of diagnostics, health systems, vector control and disease prevention, i.e. the development of vaccines.

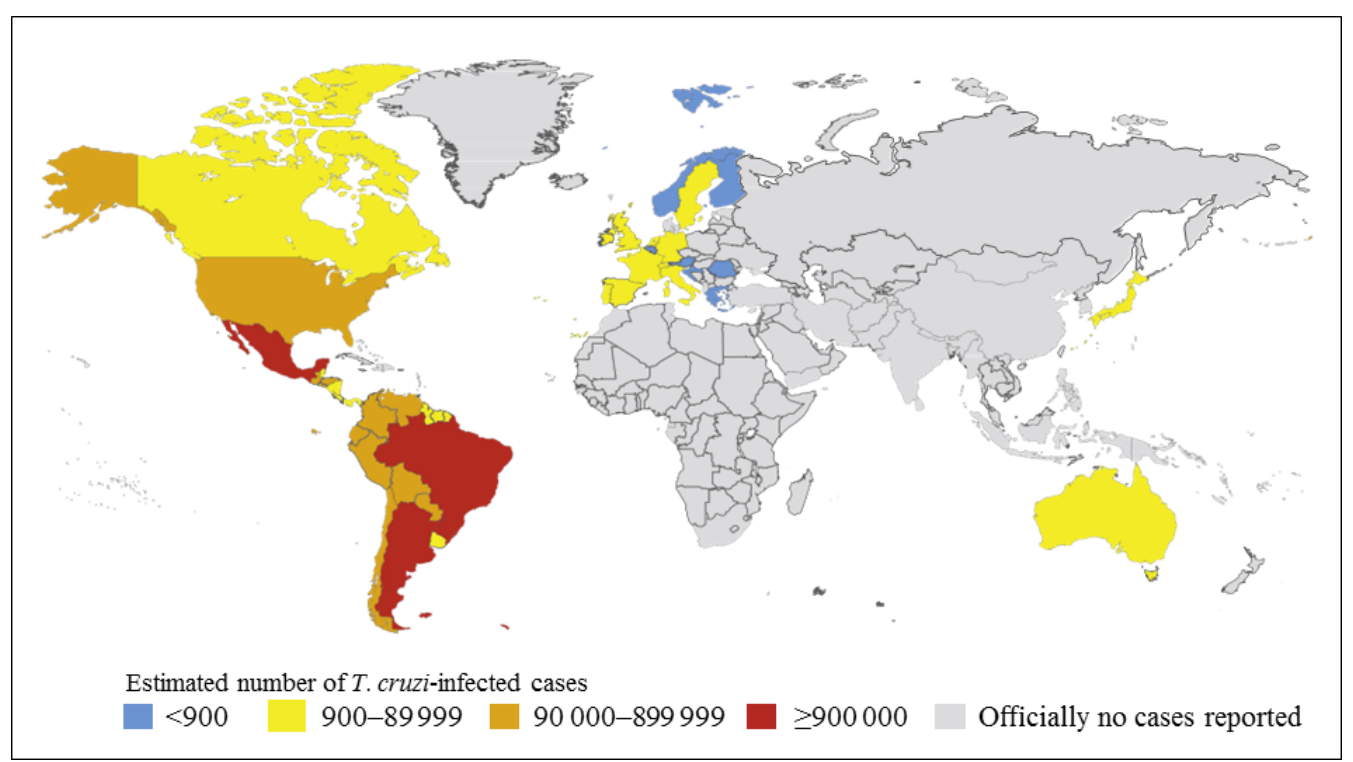

Fig. 4 Global distribution of Chagas Disease cases. Even though it is a tropical disease which originally occurred in South America, it is nowadays distributed in the four continents represented in the picture. Numbers are based on official estimates 2006 - 2010. Adapted from Trends in Parasitology.

\subsubsection{Chagas prevention}

Nowadays, several investigations are directed towards the formulation of vaccines using as antigens key peptides or proteins involved in infection establishment. In this sense, one of the main candidates is the T. cruzi trans-sialidase protein (TS) $(101,102)$. This is an enzyme of 160-200 kDa, which is anchored to the outer cellular membrane by glycosylphosphatidylinositol (GPI). It catalyzes the transfer of sialic acid from glycoproteins of the host to the $\beta$-Gal residues on mucine proteins covering the parasite membrane. In this way, T. cruzi trypomastigotes make their way into new cells upon establishment of infection, and they mask recognition sites for the immune system, often deriving in auto-immune responses. TS also helps the parasite in recognizing host cells and attaching to its sialic acids and/or $\beta$-Gal residues of their surface, through TS active site or other domains (103).

TS synthesis depends on a gene superfamily, which resulting proteins range from 60 to more than $200 \mathrm{kDa}$. Of this superfamily, only 12 are active, and they vary by their level of 
expression in the different stages of the parasite life cycle, their degree of glycosylation and the SAPA domain (shed acute phase antigen) which are tandem repeats of a 12 amino acids sequence. Since mammals lack TS and it is essential for the parasite because it cannot synthesize sialic acid the novo, this protein is currently under study as a good candidate for vaccine formulations. Different antigens have been developed based on this protein, and promising results were obtained (104).

For example, antigens TSA-1 (Trypomastigote surface antigen-1) and Tc24 (flagellar calcium binding protein of $24 \mathrm{kDa}$ ) have been thoroughly investigated and are highly immunogenic. Moreover, the protective efficacy of both has been proved in mice models and it has been reported as well that an immunotherapeutic DNA vaccine could control Chagas disease, reducing parasitemia and cardiac tissue damage $(105,106)$. Very recently, studies suggested the presence of antigen-specific memory cells induced by natural infection in seropositive patients and, in the same work, a long lasting humoral and cellular response to these antigens was evidenced, even in subjects on the chronic phase, after 10 years of infection (107).

\subsection{Subunit vaccines}

The first world-wide spread vaccine was against smallpox in the $18^{\text {th }}$ century. Upon the massive outbreak, it was noticed that infected people who survived the disease were immune. Based on this observation, a common practice of subcutaneous inoculation of smallpox virus into non-immune individuals was started. The virus was obtained from pustules of an infected person, and no regulations or thorough investigations existed for vaccination, for what considerable health risks existed, even though mortality caused by smallpox itself was significantly higher. A percentage of vaccinated patients died or contracted another disease, like tuberculosis or syphilis due to the lack of good hygiene conditions. Nevertheless, vaccination against smallpox was considered a success (108).

During the $19^{\text {th }}$ century, many others vaccines were developed, with similar strategies. For example, rabies virus extracted from dead infected rabbits were weakened by drying and then used as inocula, and killed whole cells of Vibrio cholerae were the immunization strategy against cholera $(109,110)$. Like in the former example, attenuated pathogens by drying, boiling or propagation through a foreign host are the base of "attenuated vaccines”. The principal advantages of these vaccines are their low cost and the fact that they trigger a strong, long- 
lasting immune response. Nevertheless, since the live pathogen is administered, the possibility exists that it can regain its virulence, and special attention needs to be paid with immunocompromised patients. Moreover, these vaccines need to be refrigerated, so their transportation and application in poor areas is an issue (111).

An alternative to attenuated vaccines are toxoid vaccines, which do not make use of the pathogen at all, and are the strategy of choice for vaccine development when the disease is caused by a toxin synthesized by the pathogen. Such is the case of tetanus, which is caused by a toxin secreted by Clostridium tetani and after formaldehyde treatment, minor molecular changes occur and it is converted to a toxoid. The purified product is a non-toxigenic compound capable of stimulating the immune system to generate specific antibodies that, in the event of a C. tetani infection, bind to the toxin. This complex is not able to bind to the regular toxin receptor binding sites, so the development of the disease is prevented (112). The main benefit of these vaccines is the fact that there is no risk of disease nor to revert to a virulent form. Moreover, the compounds are normally stable and less susceptible to temperatures, light and humidity. Nevertheless, they normally need an adjuvant (see below) and this usually causes local reactions at the vaccination site.

Another class of vaccines are the inactivated vaccines, like the case of hepatitis A vaccine, in which the pathogen is heated or chemically treated so that its capacity of replication is lost, but the antigens are still recognized by the immune system. The adaptive immune response is very similar to that of a toxoid vaccine, although inactivated vaccines have the advantage of having all antigens participating in the infection and therefore a broader range of antibodies is synthesized. Nevertheless, among the drawbacks of these vaccines it can be mentioned that one dose normally is not enough to trigger a strong signal to the adaptive immune system, so they are usually given in several doses and, like toxoid vaccines need to be adjuvanted (see below) (112).

Finally, the latest development in vaccines are the subunit vaccines. In this case, a particular antigen or set of antigens are provided so they are recognized by the immune system and, consequently, specific B cells are produced. Subunit vaccines can be further divided into subgroups depending on whether the antigen is a peptidic compound or a polysaccharide, which will define the kind of immune response they will generate in the patient (112). If the antigen derives from a protein (such is the case of hepatitis B and influenza vaccines) then a Tdependent response will be trigger, like for the case of toxoid vaccines. In the case of 
polysaccharide derived antigens, the response will be T-independent (e.g. the pneumococcal vaccine Pneumovax) (113). Advantages and disadvantages are similar to those of the toxoid vaccines, except that with the advances of molecular biology, strategic antigens (and adjuvants) can be designed and combined to widen the spectra of pathogen strains against which the vaccine is active (114).

\subsection{1. $L$. lactis in the industry: its potential as delivery vector}

In the last decades, a considerable amount of research has been prompted to use food grade bacteria as live vaccines. The starting point to consider a bacterium suitable for antigen delivery is its innocuity. In this sense, L. lactis classification as GRAS and QPS as previously mentioned, makes it appropriate as regards safety. L. lactis can also survive its passage through the gastrointestinal tract (GIT) for 2-3 days, without colonizing the mucosal surface of the host (115). Moreover, due to its lack of lipopolysaccharide attached to their cell membrane, it does not stimulate the host immune response.

This species in particular has considerable importance in medicine and the pharmaceutical industry, and it was in fact, the first genetically modified microorganism for the treatment of a human disease (116). For an outline with examples supporting L. lactis use in food and pharmaceutical industries, see Table I.

In 1989 Iwaki et al. proposed for the first time the use of L. lactis for the development of a mucosal vaccine against caries by use of a strain expressing a surface Enterococcus mutans protein. When the killed recombinant bacteria were orally administered in mice, salivary IgA and serum IgG response to the protein were induced (117).

Nowadays, many investigations see the use of L. lactis as an opportunity to develop competent treatments for diseases and conditions currently without a cure. For example, Inflammatory bowel diseases (IBD) like Chron's disease and ulcerative colitis are in the urgent need for the formulation of an efficient and cost effective drug treatment. Even though their causes are not completely understood, inflammation of GIT is a common consequence. The first human trial with a therapeutic bacterium, genetically engineered to treat GIT inflammation was conducted in 2006. In this work, an L. lactis secreting interleukin-10 strain gave interesting immunological results and most importantly, it was demonstrated to be safe for humans (118). 
L. lactis is therefore seen as one of the best options to deliver any molecule at the mucosa level, able to alleviate symptoms and provide the patient with a better life quality (119).

Other investigations were also developed using L. lactis as a recombinant live delivery vector, like the study performed with the tetanus toxin fragment C (TTFC). Bacteria expressing TTFC anchored to the cellular membrane were subcutaneously administered in mice, and antibody production was proven to protect mice against the lethal infection. Nasal and oral administration showed similar protection efficacies. In the former, the antigen was intracellularly overexpressed and IgG antigen production in serum protected $75 \%$ of mice (120). Nevertheless, for this example, the expression system was based on E. coli T7 bacteriophage promoter, and although it allows high expression levels of heterologous proteins of interest, it is not suitable for the final model of oral immunization. Since 2000 this system has been replaced by the nisin inducible system NICE previously described.

Since the first reported investigations about $L$. lactis as a delivery vector were made, several others were carried using this methodology for different antigens able to elicit an immune response. Mice orally immunized with recombinant L. lactis secreting Brucella abortus superoxide dismutase were reported to develop systemic and mucosal SOD-specific immune responses (121). Moreover, an oral live vaccine expressing an attenuated version of the Staphylococcal enterotoxin type B (SEB) was also reported to elicit immune protection. In this study, both intracellular and secreted versions of antigen production in the intestinal mucosa of mice showed to stimulate production of specific IgG and IgA antibodies in the serum and the feces, respectively (122). Production of specific IgA in intestinal mucosa was also obtained when BALB/c mice were orally immunized with a strain of L. lactis constitutively expressing EspB, a key protein involved in the attachment of enterohemorrhagic E. coli O157-H7 (EHEC) to enterocytes (123). Also, a vaccine against Rhodococcus equi, the main causal agent of pneumonia in foals, was developed using an L. lactis recombinant strain secreting the virulence associated protein VapA. In this study, intragastric and intranasal routes were considered, obtaining both humoral and cell-mediated immune responses (124).

As it can be appreciated, the use of L. lactis as a delivery vector of antigens synthesized in the cytosol, presented on the cellular membrane and secreted to the extracellular space, is a recently developed technology currently being exploited. Apart from its applications against bacterial pathogens of humans and animals with industrial relevance, interesting results were also obtained against the human papillomavirus and rotavirus, the most common cause of severe 
dehydrating diarrhea in children $(125,126)$. When genetically modified LAB are administered in mucosal tissues, both mucosal and systemic immune responses are triggered. Therefore, due to its non-invasive application and its rather simple manipulation without the need of highly qualified personnel, mucosal vaccines are gaining a central position in new vaccine prototypes formulation. Their production would also imply a reduction in costs, since the live microorganism synthesizes and delivers the antigen compound of interest, without the need of purification (127).

Table I. Examples of $L$. lactis strains and strategies with potential use in food and pharmaceutical industries

\begin{tabular}{|c|c|c|c|c|c|}
\hline $\begin{array}{l}\text { Parental } \\
\text { Strain }\end{array}$ & Strategy & Product & Location & Application & Reference \\
\hline NZ3950 & NICE system & L-alanine & $\mathrm{C}$ & $\begin{array}{l}\text { Food } \\
\text { sweetener }\end{array}$ & $\begin{array}{l}\text { Hols et al. } \\
1999(128)\end{array}$ \\
\hline NZ9000 & $\begin{array}{l}\text { Spontaneous } \\
\text { mutant }\end{array}$ & Vitamin B11 & $\mathrm{C}$ & $\begin{array}{l}\text { Nutritional } \\
\text { supplement }\end{array}$ & $\begin{array}{l}\text { Sybesma et } \\
\text { al, 2003(129) }\end{array}$ \\
\hline NZ9000 & $\begin{array}{l}\text { Spontaneous } \\
\text { mutants / NICE } \\
\text { system }\end{array}$ & Vitamin B2 & $\mathrm{C}$ & $\begin{array}{l}\text { "Vitamin } \\
\text { factory" }\end{array}$ & $\begin{array}{l}\text { Burgess et al. } \\
2004 \text { (130) }\end{array}$ \\
\hline IL1403 & $\begin{array}{l}\text { pILPtuf Promoter / } \\
\text { Usp45-secretion } \\
\text { signal peptide }\end{array}$ & Interleukin -6 & S & Adjuvant & $\begin{array}{l}\text { Li et al. } 2015 \\
(131)\end{array}$ \\
\hline $\begin{array}{l}\text { Not } \\
\text { informed }\end{array}$ & $\begin{array}{l}\text { pUB1000 / Usp45- } \\
\text { secretion signal } \\
\text { peptide }\end{array}$ & Insulin analogue & S & $\begin{array}{l}\text { Drug } \\
\text { delivery }\end{array}$ & $\begin{array}{l}\text { Agarwal et } \\
\text { al. 2014 } \\
(132)\end{array}$ \\
\hline MG1363 & pTREX & $\begin{array}{l}\text { Anti-TNF a } \\
\text { nanobodies }\end{array}$ & S & $\begin{array}{l}\text { Bowel } \\
\text { disease } \\
\text { treatment }\end{array}$ & $\begin{array}{l}\text { Vandenbrouc } \\
\text { ke et al. } 2010 \\
(119)\end{array}$ \\
\hline NZ9000 & NICE system & Antigen & $\begin{array}{l}\mathrm{S} \\
\mathrm{C} \\
\mathrm{A}\end{array}$ & $\begin{array}{l}\text { Rotavirus } \\
\text { vaccine }\end{array}$ & $\begin{array}{l}\text { Marelli et al. } \\
2011\end{array}$ \\
\hline IL1403 & $\begin{array}{l}\text { pILPtuf promoter / } \\
\text { USp45- secretion } \\
\text { signal peptide }\end{array}$ & Adjuvant & S & $\begin{array}{l}\text { Cancer } \\
\text { vaccine }\end{array}$ & $\begin{array}{l}\text { Kim et al. } \\
2015 \text { (133) }\end{array}$ \\
\hline MG1363 & P170 promoter & Allergen & S & Allergy & $\begin{array}{l}\text { Glenting et } \\
\text { al. } 2007 \\
(134)\end{array}$ \\
\hline NZ9000 & pSEC:LEISS & $\begin{array}{l}\text { Carcinoembryonic } \\
\text { antigen (CEA) }\end{array}$ & $\begin{array}{l}\mathrm{C} \\
\mathrm{A}\end{array}$ & $\begin{array}{l}\text { Cancer } \\
\text { vaccine }\end{array}$ & $\begin{array}{l}\text { Xiaowei et } \\
\text { al. } 2016 \\
(135)\end{array}$ \\
\hline
\end{tabular}

C: cytoplasmic - S: secreted - A: cell wall anchored 


\subsection{2. c-di-AMP as Adjuvant}

A key factor for the development of human subunit vaccines is not only to define a suitable antigen but also to select an adequate adjuvant. Vaccine adjuvants are compounds added to vaccine formulations that enhance the immunogenicity of antigens in vivo. They are further categorized based on their mechanisms of action, being divided into delivery systems (or particulate adjuvants) and immune potentiators (or immune stimulators). The former enhance the innate immune response either directly or through pattern-recognition receptors, whereas the latter help with antigen presentation and localization (136). Several advantages can be mentioned when describing the scope of adjuvants use, namely: stronger and longer immune response (especially important for weakened immune system population), improved antibody production against a broader spectrum of pathogenic strains, mucosal immune response induction, and increased biological half-life of vaccines. Hence, the amount of antigen needed is lowered, as well as the number of doses required for proper immune response generation, which consequently lowers costs of vaccination programs as well (137).

Cyclic-di-nucleotides (CDNs) have recently emerged as pathogen associated molecular patterns (PAMPs) and effective adjuvants. PAMPs and adjuvants are specifically important for the mucosal route, when antigens suffer mechanical removal and structural modifications by pH changes and enzymatic degradation. Particularly 3'5'-cyclic-di-adenosine monophosphate (c-di-AMP) was demonstrated to be a suitable candidate for vaccine adjuvant, since it has showed the capacity to activate in vitro dendritic cells of murine and human origin, one of the most important steps to stimulate the adaptive immune response. Several investigations have been carried out to test this second messenger action as an adjuvant when co-administered with an antigen of interest. Results after intra-nasal and oral immunization showed improved stimulation of immune responses at both systemic and local levels. Moreover, in vivo experiments also evidenced that c-di-AMP can promote MHC class I restricted immune response by $\mathrm{CD}^{+} \mathrm{T}$ cells, promote specific IgG and IgA production and enhance cellular proliferative responses (138). This consequently triggers the stimulation of Th1, Th2 and Th17 cells, as well as the induction of cytotoxic $\mathrm{T}$ lymphocytes. Moreover, it was recently demonstrated not only that c-di-AMP triggers a type I IFN response, but also that it downregulates it in the inductive phase of an adaptive immune response, which is a positive characteristic for its use in vaccines (139). 
In a proposed intranasal vaccine model for Chaga's disease, where c-di-AMP was coadministered with antigen TC52 (essential for the parasite T. cruzi), high specific Tc52-specific IgA titers were found in nasal lavages. Importantly, this results were significantly different from those obtained in the absence of the adjuvant c-di-AMP, and only in its presence, stronger cellular and humoral immune responses were induced (140). In another vaccine prototype based on archeaosomes as delivery system of a hepatitis $\mathrm{C}$ virus antigen, a robust $\mathrm{T}$ cell immune response was observed when adjuvanted with c-di-AMP (141). The same study also showed that a partial intranasal immunization regimen also triggers the immune response, opening the range of possibilities as regards administration.

Another non-invasive and economically favorable regimen of vaccine administration is the sublingual route. As with the intranasal, the main advantages are the simple immunization protocol along with its consequent possibility of being handled by personnel with no advanced training, which would allowed massive administration. This would provide an important benefit for example, during an epidemic outbreak. In this regard, a virosome-based vaccine against influenza virus H5N1 was tested using c-di-AMP as adjuvant, and the sublingual and intranasal inoculation as routes of administration, evidencing strong humoral and cellular responses (142).

In conclusion, the formulation of mucosal, live, subunits vaccines adjuvanted with c-diAMP and with food grade bacteria as delivery systems proposes an innovative paradigm currently under investigation. 


\subsection{Objectives}

Under the theoretical framework developed, the general objective of this thesis is to broaden the knowledge of c-di-AMP metabolism in firmicutes, and specifically in the Lactic Acid Bacteria Lactococcus lactis and Enterococcus faecalis. In addition, the aim of this work is to approach research from two perspectives: the importance of basic investigation around this newly discovered second messenger, as well as the vast application spectra it offers to the biotechnological and pharmaceutical industries.

Specific objectives set to accomplish the aforementioned general objectives are:

i) To identify and characterize c-di-AMP interaction partners in L. lactis

ii) To develop engineered strains able to modify c-d-AMP levels in L. lactis and E. faecalis and study their physiological impact

iii) To formulate a vaccine prototype by use of an L. lactis strains producing high levels of c-di-AMP

iv) To perform a first approach into the virulence of GdpP in E. faecalis 


\section{Materials and methods}

"An experiment is a question which science poses to $\mathcal{N a t u r e , ~ a n d ~ a ~}$ measurement is the recording of Nature's answer" Max Planck 


\subsection{Materials}

Lists of chemicals, commercial kits, enzymes and oligonucleotides used in this work are presented in the appendix.

\subsection{Strains and plasmids}

Bacterial strains and plasmids constructed for this work are detailed in the appendix.

\subsection{Methods}

\subsubsection{General methods}

General methods routinely used in this work are mentioned in Table II

Table II. General methods

\begin{tabular}{ll}
\hline Method & Reference \\
\hline Absorption measurement & Sambrook et al. 1989 (143) \\
DNA gel electrophoresis & Sambrook et al. 1989 (143) \\
Plasmid preparation from E. coli & Sambrook et al. 1989 (143) \\
Ligation of DNA fragments & Sambrook et al. 1989 (143) \\
Protein quantification & Bradford 1976 (144) - Lowry \\
Protein gel electrophoresis (denaturing) & 1951 (145) \\
Sequencing according to the chain termination method & Sanger et al. 1977 (147) \\
\hline \hline
\end{tabular}

\subsubsection{Storage and bacterial growth}

E. coli DH5 $\alpha$ and BL21 were grown in Luria Bertani (LB) medium at $37^{\circ} \mathrm{C}$ in aerobic conditions with vigorous shaking. E. coli LB650 and 2003 were grown under similar conditions in LB with addition of $200 \mathrm{mM} \mathrm{KCl}$ or in M9/M9mod (see Table III) supplemented with a suitable carbon source and $50 \mathrm{mM} \mathrm{KCl}$, unless otherwise stated. 
LAB were cultivated in M17 medium supplemented with $0.5 \%$ glucose (M17G), in aerobic conditions without shaking. Incubation temperatures were $30^{\circ} \mathrm{C}$ and $37^{\circ} \mathrm{C}$ for L. lactis and E. faecalis, respectively.

In all cases $-80{ }^{\circ} \mathrm{C}$ stocks prepared in $10 \%$ glycerol were propagated twice to obtain a final overnight (ON) saturated culture. This was used in turn to inoculate fresh media at an initial optical density $\left(\mathrm{OD}_{\mathrm{i}}\right)$ of 0.05 .

\section{Media composition}

Media composition is detailed in Table III. $\mathrm{pH}$ was adjusted to 7.0 or 5.5 with $\mathrm{NaOH}$ or $\mathrm{HCl}$ according to the experiment. In all cases, deionized water was used, and media were autoclaved for 20 minutes at $121{ }^{\circ} \mathrm{C}$ and 2 bar. Thermo-labile compounds were separately prepared, filter-sterilized and stored at their optimal temperature. For agar plates, $1.5 \%$ agar was added.

Table III. Growth media composition

\begin{tabular}{|c|c|c|}
\hline Medium & Component & Quantity \\
\hline Luria Bertani (LB) (143) & $\begin{array}{l}\text { Casein Peptone } \\
\text { Yeast extract } \\
\mathrm{NaCl} \\
\mathrm{dH}_{2} \mathrm{O}\end{array}$ & $\begin{array}{l}10 \mathrm{~g} \\
5 \mathrm{~g} \\
10 \mathrm{~g} \\
\text { Add to } 1 \mathrm{Lt}\end{array}$ \\
\hline $\begin{array}{l}\text { M17 } \\
\text { (Terzaghi and Sandine, 1975) } \\
\text { (148) }\end{array}$ & $\begin{array}{l}\text { Bactopeptone } \\
\text { Beef Extract } \\
\text { Yeast extract } \\
\text { Ascorbic Acid } \\
\text { Sodium } \beta \text {-glycerophosphate } \\
\mathrm{MgCl}_{2}{ }^{* 1} \\
\mathrm{MnCl}_{2}{ }^{* 1} \\
\mathrm{dH}_{2} \mathrm{O}\end{array}$ & $\begin{array}{l}10 \mathrm{~g} \\
5 \mathrm{~g} \\
2.5 \mathrm{~g} \\
2.5 \mathrm{~g} \\
19 \mathrm{~g} \\
5 \mathrm{mM} \\
1.6 \mathrm{mM} \\
\text { Add to } 1 \mathrm{Lt}\end{array}$ \\
\hline \multirow[t]{2}{*}{$\begin{array}{l}\mathrm{M}^{* 2} \\
\text { (Sambrook and Russell, 2001) } \\
\text { (149) }\end{array}$} & $\begin{array}{l}\text { M9 base 20X } \\
\mathrm{MgSO}_{4} 1 \mathrm{M} \\
\mathrm{CaCl}_{2} 0.1 \mathrm{M} \\
\mathrm{FeCl}_{3} 1 \mathrm{mM} \\
\text { Carbon source } \\
\text { Casaminoacids10\% } \\
\text { Proline } 4 \mathrm{M} \\
\text { Thiamine } 1 \mathrm{M} \\
\mathrm{dH}_{2} \mathrm{O}\end{array}$ & $\begin{array}{l}50 \mathrm{ml} \\
1 \mathrm{ml} \\
1 \mathrm{ml} \\
0.5 \mathrm{ml} \\
1 \% \\
66 \mathrm{ml} \\
10 \mathrm{ml} \\
1 \mathrm{ml} \\
\text { Add to } 1 \mathrm{Lt}\end{array}$ \\
\hline & $\mathrm{Na}_{2} \mathrm{HPO}_{4} \cdot 2 \mathrm{H}_{2} \mathrm{O}$ & $140 \mathrm{~g}$ \\
\hline
\end{tabular}




\begin{tabular}{l|l|l} 
& $\mathrm{KH}_{2} \mathrm{PO}_{4}$ & $60 \mathrm{~g}$ \\
M9 base medium & $\mathrm{NH}_{4} \mathrm{Cl}$ & $20 \mathrm{~g}$ \\
& $\mathrm{dH}_{2} \mathrm{O}$ & Add to 1 Lt \\
\hline \hline
\end{tabular}

${ }^{* 1}$ Added from $1 \mathrm{M}$ solutions, after autoclave. ${ }^{* 2}$ In M9mod medium, $\mathrm{KH}_{2} \mathrm{PO}_{4}$ is replaced for equimolar quantities of $\mathrm{NaH}_{2} \mathrm{PO}_{4}$.

When needed, growth media were supplemented with antibiotics as selective agents (Table IV), which were prepared in 1000-fold concentrated stock solutions. Deionized water was used for dissolution, except for erythromycin and chloramphenicol, in which cases ethanol was employed. Sterilization was performed by filtration and storage at $-20^{\circ} \mathrm{C}$. Antibiotics were added to media before inoculation.

Table IV. Antibiotics

\begin{tabular}{l|l|l}
\hline Bacterium & Antibiotic & Concentration \\
\hline & Ampicillin & $100 \mathrm{mg} / \mathrm{ml}$ \\
& Erythromycin & $150 \mathrm{mg} / \mathrm{ml}$ \\
& Chloramphenicol & $30 \mathrm{mg} / \mathrm{ml}$ \\
& Kanamycin & $40 \mathrm{mg} / \mathrm{ml}^{1}$ \\
& & $50 \mathrm{mg} / \mathrm{ml}^{2}$ \\
\hline \multirow{2}{*}{ L. lactis and E. faecalis } & Erythromycin & $5 \mathrm{mg} / \mathrm{ml}$ \\
& Chloramphenicol & $10 \mathrm{mg} / \mathrm{ml}$
\end{tabular}

${ }^{1}$ E. coli EC101 ${ }^{2}$ E. coli LB650

For expression or gene deletion experiments, inducers and indicators were respectively added to media (Table V).

Table V. Inducers and indicators

\begin{tabular}{ll}
\hline Compound & Stock Solution \\
\hline Arabinose & $5 \% \mathrm{p} / \mathrm{v}$ \\
IPTG & $0.5 \mathrm{M}$ \\
Nisin & $50 \mu \mathrm{g} / \mathrm{ml}$ \\
X-Gal & $40 \mathrm{mg} / \mathrm{ml}$ in (DMF) \\
\hline
\end{tabular}

\subsubsection{E. coli electroporation}

\section{E. coli electrocompetent cell preparation}


$5 \mathrm{ml}$ of a saturated $\mathrm{ON}$ culture of the E. coli strain of interest were used to inoculate 500 $\mathrm{ml}$ of $\mathrm{LB}$ medium at an $\mathrm{ODi}$ of 0.05 . Cells were incubated in a $2 \mathrm{Lt}$. Erlenmeyer at $37^{\circ} \mathrm{C}$ with vigorous shaking until OD reached 0.50 . The culture was then cooled in ice for $20 \mathrm{~min}$ and centrifuged for $5 \mathrm{~min}$ at $5000 \mathrm{rpm}$ and $4{ }^{\circ} \mathrm{C}$. Two wash steps were performed afterwards with $500 \mathrm{ml}$ ice-cold $\mathrm{d}_{2} \mathrm{O}$ and one with $10 \mathrm{ml}$ glycerol 10\%. Finally, the pellet was resuspended in $1 \mathrm{ml}$ glycerol $10 \%$ and separated in $100 \mu \mathrm{l}$ aliquots. Samples were stored $-80{ }^{\circ} \mathrm{C}$.

\section{Electroporation}

Cells were electroporated as described in Dornan and Collins, 1990, under the following conditions: $25 \mu \mathrm{F}, 2,5 \mathrm{kV}$ y $200 \Omega$. After the pulse, $1 \mathrm{ml}$ of LB was added to the electroporation cuvette and samples were incubated for 1 hour at the $37{ }^{\circ} \mathrm{C}$. Samples were plated in LB supplemented with the corresponding antibiotics and incubated ON.

\subsubsection{LAB electroporation}

\section{LAB electrocompetent cell preparation}

Strains of LAB under study were grown in M17G supplemented with 0.5 M saccharose (M17SG) and 1\% glycine. The ON saturated culture was diluted 50 times in fresh M17SG + $1 \%$ glycine. Cells were incubated at $30^{\circ} \mathrm{C}$ or $37^{\circ} \mathrm{C}$ for $L$. lactis or E. faecalis, respectively until OD reached 0.4-0.6. The culture was then harvested for 5 minutes at $5000 \mathrm{rpm}$ at $4{ }^{\circ} \mathrm{C}$. Pellets were washed in $0.5 \mathrm{M}$ ice cold saccharose $+10 \%$ glycerol using half of the culture volume. Centrifugation was performed at $6000 \mathrm{~g}$ for 8 minutes at $4{ }^{\circ} \mathrm{C}$. Cells were resuspended in $0.5 \mathrm{M}$ ice cold saccharose $+10 \%$ glycerol using $1 / 200$ of the initial culture volume.

\section{Electroporation}

LAB were electroporated as described in Dornan and Collins, 1990, under the following conditions: $25 \mu \mathrm{F}, 2,5 \mathrm{kV}$ y $200 \Omega$. After the pulse, $1 \mathrm{ml}$ of M17SG was added to the electroporation cuvette and samples were incubated for 1.5 hours at the optimal growth temperature of the strain under study $\left(30^{\circ} \mathrm{C}\right.$ for L. lactis and $37^{\circ} \mathrm{C}$ for E. faecalis). Cells were plated in M17SG supplemented with the corresponding antibiotics and incubated ON or until colonies appeared. 


\subsubsection{DNA manipulation}

\section{DNA extraction}

For plasmidic DNA extraction from E. coli, $3 \mathrm{ml}$ of a saturated ON culture was harvested at $4500 \mathrm{rpm}$ for 5 minutes. The pellet was then resuspended and processed using the Wizard ${ }^{\circledR}$ Kit (Promega) or the NucleoSpin ${ }^{\circledR}$ Plasmid Kit (Macherey-Nagel), according to manufacturer's instructions. Elution was performed with pre-warmed ${ }_{d} \mathrm{H}_{2} \mathrm{O}$ at $70{ }^{\circ} \mathrm{C}$.

Genomic DNA was similarly prepared, using the Wizard ${ }^{\circledR}$ Genomic DNA Purification Kit (Promega) or the NucleoSpin ${ }^{\circledR}$ Microbial DNA Kit (Macherey-Nagel).

When working with Gram-positive bacteria, $10 \mathrm{ml}$ of an $\mathrm{ON}$ saturated culture were harvested and the pellet was incubated with $10 \mathrm{mg} / \mathrm{ml}$ lysozyme at $30{ }^{\circ} \mathrm{C}$ for 30 minutes. The protocol was then completed using plasmidic or genomic DNA extraction kits.

\section{Polymerase Chain Reaction (PCR)}

Reactions were prepared in final volumes of 25 or $50 \mu \mathrm{l}$, using $0.2 \mu \mathrm{M}$ of specific primers and $0.5 \mu \mathrm{M}$ of dNTPs. Pfu, Taq or Phusion polymerase were used according to manufacturer's instructions. PCRs were performed in a thermocycler with 30 cycles of denaturation, annealing and elongation (Table VI). $0.5 \mu \mathrm{l}$ of plasmidic DNA or of a 10-fold dilution of genomic DNA were used as template.

Oligonucleotides were designed using VectorNTI (Invitrogen) or Geneious 7.0.2 (Biomatters) softwares and purchased from Sigma-Aldrich, Genbiotech or Invitrogen. A list of primers used in this work is provided in the appendix (Table XVII).

Table VI. PCR steps

\begin{tabular}{l|l|l}
\hline Step & Temperature $\left({ }^{\circ} \mathbf{C}\right)$ & Time \\
\hline Initial denaturation & 98.0 & $5^{\prime}$ \\
Denaturation & 98.0 & $1^{\prime}$ \\
Annealing & $52.0-59.0^{* 1}$ & $45^{\prime}$, \\
Elongation & 72.0 & Variable*2 \\
Final elongation & 72.0 & $10^{\prime}$ \\
Hold & 4.0 & hold \\
\hline \hline
\end{tabular}

${ }^{* 1}$ According to primer melting temperature (5 degrees lower)

$*^{2}$ According to enzyme processivity and product length 


\section{DNA electrophoresis}

DNA electrophoresis was performed according to Sambrook et al., 1989. Horizontal gels were prepared in TAE buffer (Table XVIII) with agarose concentrations ranging from 0.8 to $1.2 \%$ according to DNA fragment size. For DNA visualization, $1 \mathrm{X}$ GelGreen (Biotium) or HD Green Plus DNA stain (INTAS Science Imaging Instruments GmbH) was added.

Gels were submerged in TAE, samples previously stained in $1 \mathrm{X}$ sample buffer $(0,25 \%$ $(\mathrm{p} / \mathrm{v})$ bromophenol blue, 0,25 \% (p/v) xilenecyanol and $30 \%(\mathrm{p} / \mathrm{v})$ glycerol) were loaded and run at $100 \mathrm{~mA}$. DNA from $\lambda$ phage digested with EcoRI and HindIII enzymes (PBL Productos Bio-lógicos) was loaded as reference and DNA bands were visualized using a blue-light or UV transilluminator.

\section{DNA purification}

Purification of linear DNA fragments after PCR or enzymatic digestion was performed using the QIAquick ${ }^{\circledR}$ PCR purification kit (QIAGEN, Hilden), or the Gel Band Purification Kit (GE Healthcare Life Sciences) when purifying DNA from agarose gels. Protocol was followed as specified by manufacturers and final elutions were done in pre-warmed ${ }_{d} \mathrm{H}_{2} \mathrm{O}$ at $70{ }^{\circ} \mathrm{C}$.

\section{DNA enzymatic digestion}

For DNA digestion with restriction enzymes, the protocol of Sambrook (1989) was generally followed. Fast Digest or regular endonucleases purchased from Thermo scientific were used. When linearizing plasmids, dephosphorylation was performed using Calf Intestinal Alkaline Phosphatase (CIAP, Promega) or FastAP Alkaline Phosphatase (Thermoscientific). In all cases, reactions were performed following manufacturer’s instructions.

\section{Ligation of DNA fragments}

Ligase enzyme was acquired from Takara or Thermoscientific and reactions were prepared in final volumes of $20 \mu$ l. Manufacturer's protocols were followed, using in general $100 \mathrm{ng}$ of vector and a 1:5 molar ratio to calculate the volume of the fragment of interest. When ligating two DNA fragments, equimolar quantities were used. Before electroporation, ligase enzyme was heat-inactivated. 


\section{Clone screening}

In order to screen for positive clones, $3 \mathrm{ml}$ of LB (10 $\mathrm{ml}$ of M17G in the case of LAB) supplemented with the corresponding antibiotics were inoculated with a putative positive colony (normally 12 colonies were analyzed) to obtain an ON saturated culture. $50 \mu \mathrm{l}$ of these samples were harvested at room temperature at $5000 \mathrm{rpm}$ for 5 minutes. Pellets were resuspended in $25 \mu \mathrm{l}$ of EDTA $10 \mathrm{mM}$ pH 8.0 and cells were disrupted by addition of $25 \mu \mathrm{l}$ of freshly prepared crack buffer (Table VII). Samples were incubated for 5 minutes at $70{ }^{\circ} \mathrm{C}$ and cooled in ice afterwards. Next, $2 \mu \mathrm{l}$ of crack dye were added (Table VII). After 10 minutes of incubation in ice, samples were centrifuged for 5 minutes at $12000 \mathrm{rpm}$ and $4{ }^{\circ} \mathrm{C}$, and they were finally analyzed by agarose electrophoresis. Plasmids with inserts of interest were evidenced by its differential migration in the gel when compared to the empty vector.

Table VII. Clone screening solutions

\begin{tabular}{l|l|l}
\hline Solution & Composition & Concentration \\
\hline \multirow{3}{*}{ Crack buffer } & Sucrose & $0.2 \% \mathrm{p} / \mathrm{v}$ \\
& SDS & $0.5 \%$ \\
& $\mathrm{NaOH}$ & $0.2 \mathrm{~N}$ \\
\hline \multirow{2}{*}{ Crack dye } & $\mathrm{KCl}$ & $3 \mathrm{M}$ \\
& Loading dye & $1 \mathrm{X}$ \\
\hline \hline
\end{tabular}

\section{DNA Sequencing}

Plasmids and fragments of interest were sent to the DNA sequencing facility at the University of Maine, or to SeqLab Sequence Laboratories, Göttingen GmbH.

\subsubsection{Gene deletion}

After electroporation with pBVGh vector (Table XVI), cells were incubated at $30^{\circ} \mathrm{C}$ in M17G Em ${ }^{5}$ X-Gal $100 \mu \mathrm{g} / \mathrm{ml}$ agar plates.

A single blue colony was used to inoculate fresh M17G $+5 \mu \mathrm{g} / \mathrm{ml} \mathrm{Em,} \mathrm{which} \mathrm{was} \mathrm{then}$ incubated $\mathrm{ON}$ at $42{ }^{\circ} \mathrm{C}$. This culture was diluted 1/100 in fresh media, and it was incubated again at $42{ }^{\circ} \mathrm{C} \mathrm{ON}$. 
Then, cells were propagated in fresh media without antibiotic, inoculating $0.05 \%$ of the culture volume. After inoculation, samples were incubated 4 hours at $30^{\circ} \mathrm{C}$ and $\mathrm{ON}$ at $42{ }^{\circ} \mathrm{C}$. This step was repeated twice.

Serial dilutions (normally $10^{-4} ; 10^{-5} ; 10^{-6}$ ) were plated in medium with $100 \mu \mathrm{g} / \mathrm{ml} \mathrm{X-Gal}$ and no antibiotic. Plates were incubated at $42{ }^{\circ} \mathrm{C}$ for $24-48$ hours, or until colonies were distinguished.

White colonies were restricked in plates with and without antibiotic, and those which were sensitive were checked by PCR using a pair of primers hybridizing in the adjacent up and downstream genes. A set of primers amplifying an internal sequence of the gene was also used as negative control.

\subsubsection{Protein preparation and analysis}

\section{Cell disruption by French Press}

The cell body and the piston were kept in ice prior to use (French Pressure Cell, Thermo Scientific). Samples were also cooled and washed in buffer W (Table XVIII). Cell pellets were disrupted once when working with E. coli and three times when working with $L$. lactis. Pressure was 18000 psi in all cases.

\section{Cell disruption by Tissue Lyser}

$5 \mathrm{ml}$ of a LAB culture of interest were harvested for 10 minutes at $5500 \mathrm{rpm}$. Cells were washed in $1 \mathrm{ml}$ of buffer $\mathrm{W}$. Next, pellets were resuspended in lysis buffer and transferred into a $2 \mathrm{ml}$ screw-cap microvial containing $0.5 \mathrm{~g}$ of glass beads $(0.1 \mu \mathrm{m})$. Cell walls were disrupted using a mini-beadbeater-16 (Biospec, USA) in 3 cycles of 5 minutes, incubating the samples in ice for 3 minutes in each interval. Crude extracts were then processed according to the desired protocol. For larger samples, $4 \mathrm{ml}$ of sample were similarly disrupted in a $5 \mathrm{ml}$ screw-cap microvial using $0.8 \mathrm{~g}$ of glass beads. Buffers are listed in Table XVIII.

\section{Expression test of heterologous proteins in $E$. coli}

$10 \mathrm{ml}$ of a suitable medium were inoculated with the $E$. coli strains under study. When OD600 reached 0.5, 0.5 mM IPTG were added and cultures were incubated for three more hours 
at $37^{\circ} \mathrm{C}$ and continuous vigorous shaking. Samples were normalized in $100 \mu \mathrm{l}$ with equal cell amounts and spinned down in a table top centrifuge. Pellets were resuspended in $15 \mu \mathrm{l}$ of SDS loading dye and boiled for 5 minutes at $95^{\circ} \mathrm{C}$. Finally, the whole volume was loaded in an SDSPAGE gel for expression analysis.

\section{SDS Polyacrylamide Gel Electrophoresis (SDS-PAGE)}

SDS-PAGE analysis was based on Laemmli (1970) protocol. $1.0 \mathrm{~mm}$ polyacrylamide gels with concentrations ranging from $10-15 \%$ were prepared according to the molecular size of the proteins under study (Table VIII). Samples were boiled at $95{ }^{\circ} \mathrm{C}$ for 5 minutes in sample buffer. Normally, $30 \mu \mathrm{g}$ of total protein were loaded in the gel, which was submerged in running buffer. Electrophoresis was performed at room temperature, at current intensity of $20 \mathrm{~mA}$. Buffers are listed in Table XVIII.

PageRulerTM Plus Prestained Protein Ladder (ThermoFischer Scientific) or unstained Low Range Molecular Marker (BioRad) were used as size standards.

Finally, gels were analyzed by Coomassie staining, silver staining or Western Blot.

Table VIII. SDS-PAGE Gel composition

\begin{tabular}{l|c|c|c|c}
\hline Solution & \multicolumn{3}{|c|}{ Separating gel } & $\begin{array}{c}\text { Stacking } \\
\text { gel }\end{array}$ \\
\hline $\begin{array}{l}\text { Acrylamide (Stock } 30 \% \\
\text { acrylamide-bisacrylamide 19:1) }\end{array}$ & $10 \%$ & $12 \%$ & $15 \%$ & $5 \%$ \\
\hline Tris-HCl (Stock 1.5 M) & \multicolumn{3}{|c|}{$375 \mathrm{mM}^{* 1}$} & $187.5 \mathrm{mM}^{* 2}$ \\
\hline SDS (Stock 20 \%) & \multicolumn{3}{|c}{$0.1 \%$} \\
\hline APS (Stock 10 \%) & \multicolumn{3}{|c}{$0.04 \%(\mathrm{v} / \mathrm{v})$} & $0.1 \%(\mathrm{v} / \mathrm{v})$ \\
\hline TEMED &
\end{tabular}

${ }^{* 1}$ Tris $\mathrm{HCl} \mathrm{pH} 8.8{ }^{* 2}$ Tris $\mathrm{HCl} \mathrm{pH} 6.8$

\section{Coomassie Staining}

For Coomassie staining after SDS-PAGE, gels were incubated in Coomassie R-250 for 30 minutes at room temperature in a rocking shaker. For protein visualization, gels were afterwards boiled or incubated in destaining solution containing $20 \% \mathrm{v} / \mathrm{v}$ ethanol and $5 \% \mathrm{v} / \mathrm{v}$ acetic acid until protein bands were evidenced. 


\section{Silver staining}

When protein amounts were low, visualization was accomplished by silver staining which allows a more sensitive detection (up to $1 \mathrm{ng}$ of protein) (150). For this, the following protocol was followed:

- Fixation: the gel was incubated in fixing solution, at least during 1 hour

- Wash: 3 times with $50 \%$ ethanol, during 20 minutes

- Reduction: Performed in $0.02 \%$ (p/v) $\mathrm{Na}_{2} \mathrm{~S}_{2} \mathrm{O}_{3}$

- Wash: 3 times with $\mathrm{dH}_{2} \mathrm{O}$ for 20 seconds

- Impregnation: the gel was incubated for 15'-25' in impregnating solution

- Wash: 3 times with $\mathrm{dH}_{2} \mathrm{O}$ for 20 seconds

- Development: developing solution was used until bands appeared

- Wash: 3 times with $\mathrm{dH}_{2} \mathrm{O}$ for 20 seconds

- Development was stopped by incubation in stopping solution

Table IX. Silver staining solutions

\begin{tabular}{l|l|l}
\hline Solution & Composition & Concentration \\
\hline \multirow{3}{*}{ Fixing } & Methanol (100 \%) & $50 \%(\mathrm{v} / \mathrm{v})$ \\
& Acetic acid (100\%) & $12 \%(\mathrm{v} / \mathrm{v})$ \\
& Formaldehyde (37\%) & $0.1 \%(\mathrm{v} / \mathrm{v})$ \\
\hline \multirow{2}{*}{ Impregnating } & $\mathrm{AgNO}_{3}$ & $0.2 \%(\mathrm{p} / \mathrm{v})$ \\
& Formaldehyde (37\%) & $0.037 \%(\mathrm{v} / \mathrm{v})$ \\
\hline \multirow{3}{*}{ Developing } & $\mathrm{Na}_{2} \mathrm{CO}_{3}$ & $12 \%(\mathrm{p} / \mathrm{v})$ \\
& $\mathrm{Na}_{2} \mathrm{~S}_{2} \mathrm{O}_{3}$ & $0.0004 \%(\mathrm{p} / \mathrm{v})$ \\
& $\mathrm{Formaldehyde} \mathrm{(37 \% )} 0.05 \%(\mathrm{v} / \mathrm{v})$ \\
\hline STOP & $\mathrm{Na}_{2}$-EDTA & $1.86 \%(\mathrm{p} / \mathrm{v})$ \\
\hline \hline
\end{tabular}

\section{Western blot}

After running an SDS-PAGE, the gel was incubated in transfer buffer (Table X) for 5 minutes. Proteins were then transferred to a nitrocellulose membrane (Schleicher \& Schuell BA85, 0,45 $\mu \mathrm{m}$ ) using a mini-protean two cell unit (Bio-Rad, Hercules, CA, USA) (the sponges, Whatman paper and the membrane were also previously incubated in transfer buffer). Protein transfer was performed at $100 \mathrm{~mA}$ for 2 hours or at $20 \mathrm{~mA}$ for 12 hours, and efficiency was assessed by staining with Pounceau Red S (Sigma, USA). After this, the membrane was treated according to the following protocol: 
- Wash: Rinse with $\mathrm{dH}_{2} \mathrm{O}$ and incubate twice in TBS for 10 minutes

- Block: Incubate 1 hour in TBS + $5 \%$ milk

- Wash: Twice in TBS + $0.05 \%$ Tween and $0.2 \%$ Triton for 10’ minutes

- Wash: Once in TBS for 10 minutes

- Incubate 1 hour with Anti-His (1/200) in TBS + $5 \%$ milk

- Wash: Twice in TBS + $0.05 \%$ Tween and $0.2 \%$ Triton for 10 minutes

- Wash: Once in TBS for 10 minutes

- Incubate 1 hour with secondary antibody in TBS + $5 \%$ milk

- Wash: Four times in TBS + 0,05 \% Tween + 0,2 \% Triton for 10 minutes

- Wash: Once with phosphatase buffer for 10 minutes

- Incubate in the dark in $10 \mathrm{ml}$ phosphatase buffer + $33 \mu \mathrm{l}$ 5-bromo-4-chloro-3-indoyl phosphate (BCIP; $50 \mathrm{mg} / \mathrm{ml}$ in $100 \% \mathrm{DMF})+66 \mu \mathrm{l}$-nitroblue tetrazolium chloride (NBT; 50 mg/ml en 70 \% DMF)

Table X. Western Blot Solutions

\begin{tabular}{l|l|l}
\hline Solution & Composition & Concentration \\
\hline \multirow{3}{*}{ Transfer } & Methanol (100 \%) & $20 \%(\mathrm{v} / \mathrm{v})$ \\
& Tris & $20 \mathrm{mM}$ \\
& Glycine & $150 \mathrm{mM}$ \\
\hline \multirow{3}{*}{ TBS 10X } & Tris & $50 \mathrm{mM}$ \\
& $\mathrm{NaCl}$ & $150 \mathrm{mM}$ \\
& Adjust pH to 7.5 & \\
\hline \multirow{3}{*}{$\begin{array}{l}\text { Phosphatase } \\
\text { buffer }\end{array}$} & Tris & $100 \mathrm{mM}$ \\
& $\mathrm{NaCl}$ & $100 \mathrm{mM}$ \\
& $\mathrm{MgCl}_{2}$ & $10 \mathrm{mM}$ \\
\hline \hline
\end{tabular}

\subsubsection{Microplate growth curves}

All microplate growth curves were performed in a final volume of $200 \mu \mathrm{l}$. ON saturated cultures were used to inoculate fresh media at an $\mathrm{OD}_{\mathrm{i}}$ of 0.05 . When $\mathrm{OD}_{600}$ reached 0.5, cells were harvested and resuspended to inoculate microplates at an $\mathrm{OD}_{\mathrm{i}}$ of 0.05 . Reads were performed every 15 minutes for 16 hours, at the optimal growth temperature of the microorganism under study. 


\section{Growth curves on minimal salt media}

When curves were performed in minimal salt M9mod media, strains derived from E. coli LB650 were propagated twice from $-80^{\circ} \mathrm{C}$ stocks in M9mod supplemented with $50 \mathrm{mM} \mathrm{KCl}$ and $1 \%$ glucose. The ON saturated culture thus obtained was used to inoculate fresh M9mod media supplemented with $10 \mu \mathrm{M}$ IPTG and $0.1 \mathrm{mM} \mathrm{KCl}$ for strains E. coli LB09 and LB10, 50 $\mathrm{mM} \mathrm{KCl}$ for strain LB11 and $100 \mathrm{mM} \mathrm{KCl}$ for LB08 and LB12. When OD reached 0.5, cultures were harvested and incubated for 1 hour in the initial volume of fresh media, this time without $\mathrm{KCl}$ supplementation. Afterwards, cells were harvested and washed three times with fresh media with no $\mathrm{KCl}$. These samples were then used to inoculate fresh M9mod media in microplates supplemented with $1 \%$ glucose, $10 \mu \mathrm{M}$ IPTG and different $\mathrm{KCl}$ concentrations under study. Microplates were incubated at $37^{\circ} \mathrm{C}$ with continuous orbital shaking.

\section{Co-expression of Kup transporters and c-di-AMP}

M9mod supplemented with 0,2 \% Glycerol and $10 \mathrm{mM} \mathrm{KCl}$ was inoculated with $-80{ }^{\circ} \mathrm{C}$ stock strains under study and propagated twice. With the ON saturated cultures, $10 \mathrm{ml}$ of fresh media were inoculated at $\mathrm{OD}=0.05$. When strains reached $\mathrm{OD}=0.5$, cultures were harvested and resuspended in same volume of M9mod supplemented with 0,2 \% glycerol and no $\mathrm{KCl}$. Samples were incubated at $37{ }^{\circ} \mathrm{C}$ for an hour, after which 2 wash steps with no potassium supplementation were performed. These washed samples were used for microplate inoculation supplemented with $0,1 \mathrm{mM} \mathrm{KCl}$ and $2.5 \mu \mathrm{M}$ IPTG. Genes cloned in pBAD33 vector were induced with $0.005 \%$ L-arabinose when indicated. Growth curves were obtained as previously described.

\section{Overproduction of proteins in E. coli BL21}

Overexpression genes in E. coli BL21 was carried as follows. Overnight cultures of strains under study were diluted to an OD600 $=0.05$ in 2 Lt. of fresh LB media supplemented with $100 \mu \mathrm{g} / \mathrm{ml}$ ampicillin. When an OD600 of 0.5 was reached, $0.5 \mathrm{mM}$ IPTG was added to the cultures, which were further incubated for $3 \mathrm{~h}$.

\section{Overproduction of TScf in L. lactis}

Expression of TScf was carried as follows. Overnight cultures of recombinant L. lactis were diluted to an $\mathrm{OD}_{600}=0.05$ in $3 \mathrm{Lt}$. of fresh M17G medium supplemented with the 
corresponding antibiotics. Cultures were incubated at $30^{\circ} \mathrm{C}$ until an $\mathrm{OD}_{600}$ of 0.5 was reached, when $5 \mathrm{ng} / \mathrm{ml}$ of nisin (Sigma, USA) was added to the cultures. Samples were further incubated for $3 \mathrm{~h}$.

\section{Purification of His $6 \mathrm{x}$-tagged proteins from $L$. lactis}

After induction, L. lactis samples were collected by centrifugation and resuspended in lysis buffer. Cells were then lysed with a tissue lyser as previously described. The lysate was clarified by centrifugation, and $\mathrm{NaH}_{2} \mathrm{PO}_{4}$ and imidazole were added to a final concentration of $100 \mathrm{mM}$ and $5 \mathrm{mM}$, respectively, $\mathrm{pH}$ was adjusted to 8 .

Clarified lysates were applied to a $\mathrm{Ni}^{2+}$-NTA affinity column (Qiagen) previously equilibrated with lysis buffer and incubated at room temperature for 1 hour to allow binding. Then the protein was refolded by successive in-column incubations with buffer $\mathrm{C}$ containing decreasing concentrations of urea ranging from $6 \mathrm{M}$ to $0 \mathrm{M}$. The column was then washed with buffer $\mathrm{C}$ plus $25 \mathrm{mM}$ imidazole and the protein was eluted from the column with buffer $\mathrm{C}$ with $500 \mathrm{mM}$ imidazole. The purified protein was dialyzed against PBS plus $5 \%$ glycerol; aliquots were kept at $-80{ }^{\circ} \mathrm{C}$. Buffers are listed in Table XVIII.

\section{Purification of $\mathrm{His}_{6 \mathrm{X}}$-tagged proteins from $E$. coli}

E. coli induced pellets from 2 Lt. cultures were washed in buffer $\mathrm{W}$ and cells were resuspended in buffer C, to be disrupted via French Press as previously described. Samples were then centrifuged 10 minutes at $5000 \mathrm{rpm}$ and remaining insoluble particles were removed by ultracentrifugation at $4{ }^{\circ} \mathrm{C}$ and $35000 \mathrm{rpm}$ for 1 hour. For purification of recombinant His6xtagged proteins the supernatant fraction was loaded onto a bed of $1000 \mu \mathrm{l}$ of $\mathrm{Ni}^{2+}$-NTA resin (IBA) in a Poly-Prep Chromatography Column (BioRad) that had been pre-equilibrated in buffer C. After applying the cell extract, the column was washed 5 times with $5 \mathrm{ml}$ of buffer $\mathrm{C}$ containing $10 \mathrm{mM}$ imidazole. The elution was performed in steps of $5 \mathrm{ml}$, with increasing concentrations of imidazole (30 mM, 50 mM, 100 mM, $200 \mathrm{mM}$ and $500 \mathrm{mM}$ ). Fractions were analyzed by SDS-PAGE and those were a band evidenced the presence of the protein of interest were pooled and concentrated by centrifugation in Vivaspin turbo 15 columns (Sartorius) for 15 minutes at $4.000 \mathrm{rpm}$ and $8^{\circ} \mathrm{C}$. 
Protein concentration was determined by Bradford assay and samples were further purified and analyzed via Size-exclusion chromatography (SEC). Buffers are listed in Table XVIII.

\section{Size exclusion chromatography}

The experiments were performed at room temperature in a HiLoad 16/600 Superdex 200 pg using an ÄKTAprime plus chromatography system (GE Healthcare Life Sciences). Purified protein was applied to the column previously equilibrated with $20 \mathrm{mM}$ Tris- $\mathrm{HCl}, 50$ $\mathrm{mM} \mathrm{NaCl}$, pH 8.0 buffer. The program was set to $1 \mathrm{ml} / \mathrm{min}$ and several elution fractions were recovered, where protein concentration was measured by a spectrophotometer coupled to the column. A calibration run was performed with molecular weight standards and related to their elution volumes for both column sizes. The elution volume is directly proportional to the $\log _{10}(\mathrm{Mw})$.

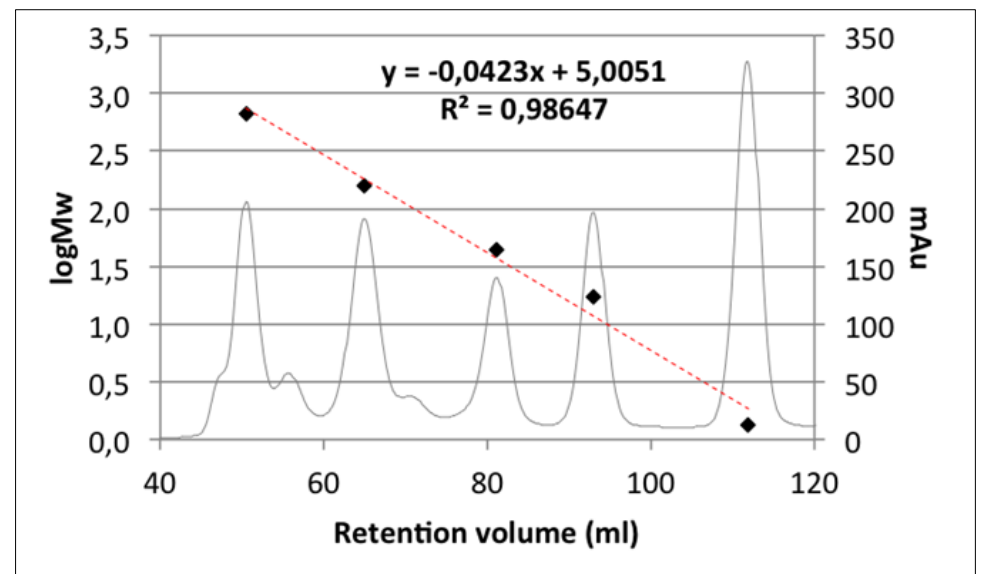

Fig 5 Standard curve in a HiLoad 16/600 Superdex $200 \quad$ pg chromatography column. Proteins of different molecular weight were injected into the size-exclusion chromatography column. The grey line, corresponding to the secondary vertical axis represents the spectrum of elution. The logarithm of the molecular weight of each protein was plotted against its retention volume. The standard curve was then used to interpolate molecular weight from experimental retention times.

Table XI. Standard values for HiLoad 16/600 Superdex 200 pg

\begin{tabular}{c|c|c}
\hline $\mathrm{Mw}(\mathrm{KDa})$ & $\log _{10}(\mathrm{Mw})$ & $\begin{array}{c}\text { Retention } \\
\text { volume (ml) }\end{array}$ \\
\hline 670 & 2.83 & 50.45 \\
158 & 2.20 & 64.92 \\
44 & 1.64 & 81.19 \\
17 & 1.23 & 93.02 \\
1.35 & 0.13 & 111.92 \\
\hline
\end{tabular}




\subsubsection{Protein pull-down experiment with strep-tagged magnetic beads}

Cultures of L. lactis IL1403 were grown in M17G medium until a final OD600 of 0.5, when cells were harvested at $10000 \mathrm{rpm}$ for 5 minutes. Pellets were resuspended in $2 \mathrm{ml}$ of CP1 buffer. This suspension was disrupted using French press and centrifuged 10 minutes at 10000 rpm and $4{ }^{\circ} \mathrm{C}$. The supernatant was split in two fractions of equal volume and centrifuged for 1 hour at $68000 \mathrm{rpm}$ and $4{ }^{\circ} \mathrm{C}$. Protein quantification of the supernatant was then performed via Bradford assay.

$1.2 \mathrm{mg}$ of protein was then taken to a final volume of $1 \mathrm{ml}$ CP1 buffer, with addition of glycerol $10 \%, 0.004 \%$ EDTA and $0.5 \mu \mathrm{g} / \mathrm{ml}$ BSA. This sample was then incubated for 0.5 hour at room temperature in a vortex shaker with the + and - beads (see below).

Strep-tactin beads were equilibrated with CP2 buffer. After equilibration, half of the tubes were incubated with biotinylated c-di-AMP in a vortex shaker for 15 minutes at room temperature (+ samples). Finally, the beads were washed twice with CP2.

After incubation with pre-equilibrated beads, samples were washed four times to get rid of unbound proteins and finally, elution was done with $50 \mu \mathrm{l}$ buffer $\mathrm{E}$ at room temperature for 15 minutes in a vortex shaker.

Protein elution was analyzed by SDS-PAGE and silver staining. Both + and - lanes were sent for peptide identification at the proteomic department of the Ernst Moritz Arndt University, Greifswald (Prof. Uwe Völker). Buffers are listed in Table XVIII.

\subsubsection{Differential radial capillary action of ligand assay (DrACALA)}

Genes of interest were cloned in vector pWH844 using strain E. coli BL21 as host. An induction test was then performed as previously described to check gene expression (Zuzanna Grubek Master Thesis, 2016). Plasmids under study were finally sent to Prof. Vincent Lee (Department of Cell Biology and Molecular Genetics, University of Maryland, U.S.A) for the evaluation of specific interactions between their corresponding encoded proteins and c-di-AMP via the DrACALA assay. 


\subsubsection{Determination of c-di-AMP intracellular levels}

$20 \mathrm{ml}$ cultures of L. lactis IL1403 were grown in M17G medium. When samples reached an $\mathrm{OD}_{600}$ of 0.50 , cells were harvested at $4{ }^{\circ} \mathrm{C}$ and $5000 \mathrm{rpm}$, and quickly frozen in liquid nitrogen. Two additional samples of $1 \mathrm{ml}$ were taken for normalization purposes. Samples were collected and stored at $-20{ }^{\circ} \mathrm{C}$ until c-di-AMP extraction was performed. For this, pellets were resuspended in $150 \mu \mathrm{l} 2 \mathrm{mg} / \mathrm{ml}$ lysozyme in TE buffer and incubated for 30 minutes at $25{ }^{\circ} \mathrm{C}$ and $750 \mathrm{rpm}$. Afterwards, samples were frozen in liquid nitrogen and boiled at $95{ }^{\circ} \mathrm{C}$ for 10 minutes. First, an extraction with $800 \mu$ acetonitrile:methanol 1:1 was done, after which two more extractions with $200 \mu \mathrm{l}$ acetonitrile:methanol:water 2:2:1 were performed. Supernatants were collected and dried in a Speedvac at $40^{\circ}$ C. Pellets were sent to Prof. Volkhard Kaever from the Medizinische Hochschule, Hannover for c-di-AMP quantification via mass spectrometry. Final data was normalized with respect to the amount of protein present in the sample, determined via Bradford assay.

\subsubsection{Bis(p-nitrophenyl)phosphate assay.}

Bis(p-nitrophenyl)phosphate (BNPP) assay was performed according to Bai et al. 2013 (68). Briefly, the following reaction is set up in a final volume of $100 \mu \mathrm{l}$ :

$\begin{array}{ll}\text { Putative phosphodiesterase } & 0.1 \mu \mathrm{M} \\ \text { Tris-HCl pH } 7.5 & 100 \mathrm{mM} \\ \mathrm{Mn} 2 \mathrm{Cl} & 100 \mu \mathrm{M} \\ \mathrm{NaCl} & 10 \mathrm{mM} \\ \mathrm{BNPP} & 2 \mathrm{mM} \\ \mathrm{d}_{2} \mathrm{O} & \text { for } \mathrm{V}_{\mathrm{f}}=100 \mu \mathrm{l}\end{array}$

Reactions were loaded in microplates and were followed in a microplate reader at 30 ${ }^{\circ} \mathrm{C}$ and $\lambda=410 \mathrm{~nm}$ during 4 hours. Incubation was performed with minimal reading intervals and medium shake. 


\subsubsection{Light microscopy}

For microscopic analysis of $L$. lactis samples, cells were grown in M17G medium at $30^{\circ}$ $\mathrm{C}$ to the desired $\mathrm{OD}_{600}$. A volume of $0.3 \mu \mathrm{l}$ was placed on a slide covered with a thin layer of $1 \%$ agarose prepared in $\mathrm{d}_{2} \mathrm{O}$, and when dried, a coverslip was placed on the sample. Pictures were taken using the AxioImager M2 equipped with a digital camera AxioCam MRm and the AxioVision Rel 4.8 software for image uptake (Zeiss). The objectives used ECPlanNEOFLUAR 100X/1.3 (Zeiss).

\subsubsection{Survival analysis in Galleria mellonella}

Survival experiments in G. mellonella were performed as follows: larvae were selected according to their weight, which was between 0.18 and 0.35 grams. 32 individuals per group were then harvested $\mathrm{ON}$ at $30^{\circ} \mathrm{C}$. Each group was inoculated with bacterial suspensions made in PBS at a concentration of $9.10^{6} \mathrm{CFU} /$ larva. Inoculation site was the fifth proleg.

After inoculation, larvae were monitored at 2-4 hours intervals for 72 hours. Then, data was plotted in Kaplan-Meier curves using SigmaPlot 12 software and employing LogRank and Holm-Sidak tests, for multiple comparisons. P value was set in 0.05 (151).

\subsubsection{Immunological experiments}

\section{Preparation of live bacterial inoculum and immunization protocol}

2 Lt. cultures of M17G supplemented with the corresponding antibiotics and $15 \mathrm{ng} / \mathrm{ml}$ of nisin were inoculated with $L$. lactis strains of interest at $\mathrm{OD}_{\mathrm{i}}=0.05$. Samples were incubated at $30{ }^{\circ} \mathrm{C}$ until $\mathrm{OD}_{600}$ reached 0.5 . Cells were then centrifuged at $5000 \mathrm{rpm}$ and $4{ }^{\circ} \mathrm{C}$ for 10 minutes and washed with PBS buffer, to be finally resuspended in PBS plus $10 \%$ glycerol. CFU were determined in the samples to check concentrations of the range $2-4.10^{9} \mathrm{CFU} / 100$ $\mu \mathrm{l}$ inoculum. In addition, an expression test was performed to corroborate the presence of TScf.

\section{Mice inoculation}

BALB/c female mice, aged 6 weeks, were acquired and housed in HEPA-ventilated racks at $21-22{ }^{\circ} \mathrm{C}$ and $68 \%$ humidity at the animal facility of the CIPREB (Center for Research and Production of Biological Reagents, School of Medicine, UNR). Animals had free access to 
food and water and were maintained under a 12 hours light/dark period. All protocols for animal studies were performed in collaboration with Dra. Ana Rosa Pérez and Dr. Iván Marcipar groups from the Instituto de Investigación Clínica y Experimental de Rosario (IDICER CONICET-UNR) and the Laboratorio de Tecnología Inmunológica, Facultad de Bioquímica y Ciencias Biológicas, UNL (Universidad Nacional del Litoral), respectively. Moreover, these experiments were approved by the Bioethics and Animal Care \& Use Committees according to Institutional guidelines.

Briefly, mice were immunized by oral route by three successive doses separated by twoweek intervals by gavage administration. The bacterial dose administered was set as a quantity of bacteria expressing $10 \mu \mathrm{g}$ of TScf $\left(0.3-1.10^{6} \mathrm{CFU}\right)$. Similar quantities of bacteria expressing the TScf antigen alone, the CdaA alone or the empty vector were administrated in parallel. For comparative purposes, a group of animals was subcutaneously immunized in parallel with 10 $\mu \mathrm{g}$ of purified TScf adjuvanted with $3 \mu \mathrm{g}$ of ISPA (“Gold standard” group), an ISCOMATRIX type adjuvant developed by Dr. Marcipar group (152).

\section{Cellular response (Delayed hypersensibility test)}

To test cellular response, mice were challenged with $5 \mu \mathrm{g}$ of purified TScf by intradermal injection in the right footpads 12 days after the last immunization. The thickness of hind footpads was measured before and 24, 48 and 72 hours after the antigen injection with a digital Vernier caliper. Results of the delayed hypersensitivity test (DHT) were expressed as the difference in thickness of footpads after and before the inoculation. 
3. Results

"Nothing in life is to be feared, it is only to be understood. Now is the time to understand more, so that we fear less" Marie Curie 


\section{1. c-di-AMP and potassium uptake in L. lactis IL1403}

\subsubsection{Identification of c-di-AMP interaction partners in L. lactis IL1403}

In an attempt to identify new interaction partners of c-di-AMP in L. lactis IL1403, a protein pull-down experiment was performed (50). As described in Materials and Methods, cell extracts of this strain were incubated with strep-tagged magnetic beads previously coupled to biotinilated c-di-AMP (+ sample). A sample incubated with the beads without previous treatment was used as negative control (- sample). Elutions were analyzed by SDS-PAGE and silver staining (Fig. 6, I) and both lanes were sent for peptide identification by mass spectrometry. A list of 151 different proteins was obtained, with the ratio of their presence in the + lane with respect to the - lane. For the list of peptides obtained after the pull-down experiment, see Table XIV in the Appendix.

It is important to mention that biotinilated proteins would also bind to the strep-tag in the - sample, which is probably why pyruvate carboxylase was identified almost 50 times more represented in the - lane. On the other hand, secondary interaction partners, i.e. proteins interacting with c-di-AMP binding proteins, could also be eluted depending on the strength of the interaction. Moreover, if the interaction to c-di-AMP is not strong enough, some binding partners could also be washed out, or found with a low +/- ratio. To evaluate all these possibilities, a bibliographic search was carried out, although the vast majority of the proteins on the list are either uncharacterized, or no studies about them have so far been reported. Since there is no information available about which proteins are biotinilated, nor which are essential in L. lactis (which could lead to the essential role of c-di-AMP in this bacterium), an initial classification was made by use of the UniProt online resource.

Fig. 6, II shows a functional classification of the 130 peptides with the highest +/- ratio, based on available information and amino acid sequence homology to previously studied proteins from other bacteria. As the pie chart shows, the most represented groups include transport proteins comprising a predicted glutamine ABC transporter, a glycine-betaine transporter, four putative $\mathrm{ABC}$ transporters and two uncharacterized proteins. Also, a considerable amount of proteins related to DNA metabolism were identified, such as the DNA polymerase III, subunits $\beta$ and $\tau$, the DNA mismatch repair proteins MutS and MutL, and the predicted proteins RecG (DNA helicase), RecN (DNA repair) and RecA (DNA 
repair/homologous recombination). Finally, groups of proteins related to amino acid and cell wall metabolism were also among the most represented, including predicted amino acid synthesizing enzymes and proteins involved in the formation of the septal ring and cell division (EzrA, FtsA and FtsE, which was proved to affect translocation of $\mathrm{K}^{+}$in E. coli (37)).

Considering that actually most of the proteins showed a great variety of predicted functions and therefore they were collected under "Miscellaneous proteins", no clear tendency could be distinguished. Altogether, for the subsequent specific interaction assay, the 23 peptides with the highest +/- ratio were selected. In other words, this selection established a cut off of proteins which were at least eight times more represented in the + lane than in the - lane (Table $\mathrm{XII})$. Due to the proximity of the ratio value to the set cut off, the uncharacterized protein with an accession number Q9CEI1_LACLA, and the predicted septation ring formation regulator EzrA were as well added to the list.

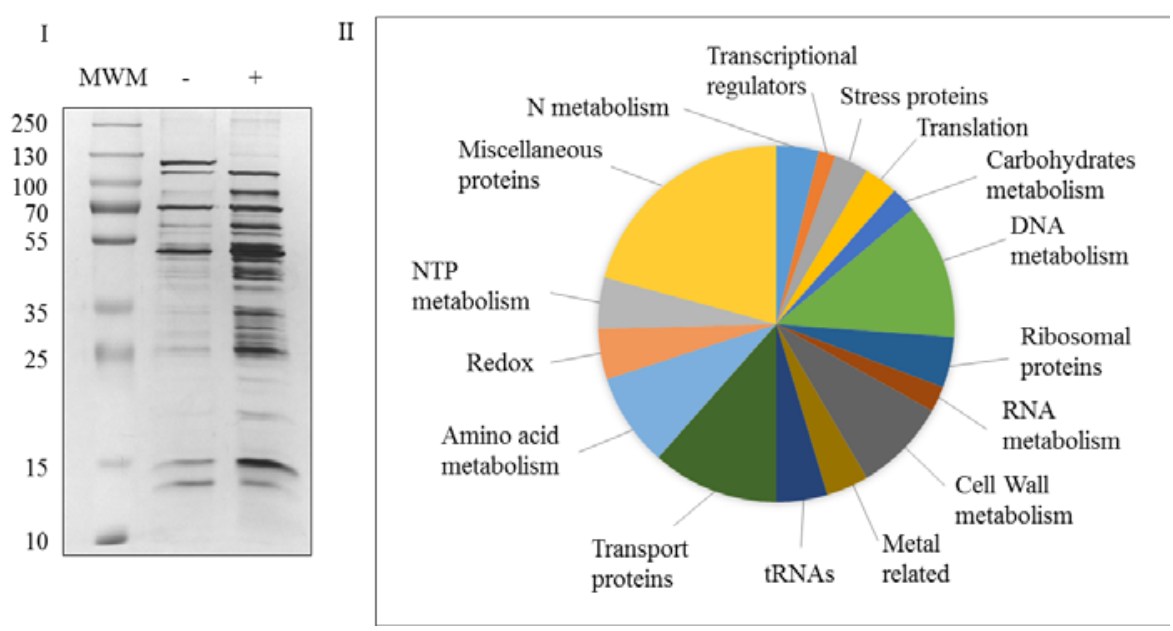

Fig. 6 Protein pull-down for identification of c-di-AMP interaction partners. I. SDS-PAGE after pull-down experiment with strep-tagged magnetic beads (see Materials and Methods). Bands were visualized by silver staining. + lane: sample eluted from beads previously treated with biotinilated c-di-AMP. - lane: sample eluted from beads with no previous treatment. MWM: Molecular weight marker (PageRulerTM Plus Prestained Protein Ladder). This experiment was performed in three independent replicates. Both lanes were sent for peptide identification. II. Functional classification of proteins obtained after MS/MS detection. Protein function was assigned based on reported research and sequence homology to previously studied proteins.

Furthermore, at the time this experiment was performed, an increasing amount of evidence was directing the essential role of $c d a A$ (and consequently c-di-AMP) towards its relation to potassium metabolism (153). Consequently, a search for genes coding for putative potassium transporters, and proteins potentially related to potassium homeostasis in L. lactis IL1403 was carried out. 
When looking for potassium transporters encoded in L. lactis IL1403 genome by use of the free online software UniProt (UniProtKB, UniRef and UniParc databases), proteins KupA, KupB and YrbD are shown as results. These three proteins are inferred from homology since there is currently no reported research about them. The first two belong to the KT/KUP/HAK family (see below), whereas YrbD could be a voltage gated channel. Since neither potassium metabolism, nor its relation to c-di-AMP has been thoroughly investigated in L. lactis, the possibility of this second messenger interacting as well with potassium channels was considered. Thus, these proteins were added to the list along with the putative voltage gated potassium channel proteins YjdJ and YncB, with more than $60 \%$ identity to the potassium efflux system KefA from other Lactococcus species.

Proteins with high homology for members of the Trk/Ktr/HKT family were also evaluated. Interestingly, even though putative homologous proteins to B. subtilis KtrA were found in some members of L. lactis species, it is not present in IL1403 nor in MG1363 strains studied in this work. Moreover, even though the kim riboswitch was already reported to be absent in Lactococcus (154), the presence of the associated potassium transporter KimA, or possible related proteins was also considered. No KimA homologues were found encoded in $L$. lactis genome, but the protein blast showed certain homology to an amino acid permease, LysP. Given the recent associations of c-di-AMP to amino acids metabolism and osmoregulation previously described in the introduction $(62,155)$, both LysP and LysQ were also included in the list of putative c-di-AMP interaction partners.

It was also reported that in L. monocytogenes a mutant strain for the only c-di-AMP synthesizing enzyme was obtained in rich media thanks to loss of function mutations occurring in genes clusters oppABCDF (involved in oligopeptide import) or the $g b u A B C$ (involved in glycine-betain import) (60). Therefore, the homologous Opp protein of L. lactis IL1403, which is expected to function as an oligopeptide permease, was added as well to the list.

Lastly, and due to the close connection between potassium and sodium homeostasis, Nah protein, homologous to a cation:proton antiporter was also selected. In conclusion, a final selection of 34 genes (Table XII) was then cloned in the E. coli expression vector pWH844 (Zuzanna Grubek Master Thesis, 2016). After checking expression of all genes using strain $E$. coli BL21 as host, the corresponding plasmids were sent to confirm c-di-AMP specific interaction to the respectively encoded proteins via differential radial capillary action of ligand assay (DrACALA) (see Materials and Methods). 
Table XII. Gene selection for DrACALA analysis

\begin{tabular}{|c|c|c|}
\hline Gene & Putative function & Reference \\
\hline dnaH & DNA polymerase III, subunits $\beta$ and $\tau^{1}$ & Bolotin et al. 2011 \\
\hline ezrA & Septation ring formation regulator ${ }^{1}$ & Bolotin et al. 2011 \\
\hline ftsE & Control of cell wall synthesis; $A B C$ transporter ${ }^{1}$ & Bolotin et al. 2011 \\
\hline$g \ln P$ & $\begin{array}{l}\text { Glutamine ABC transporter permease and substrate binding } \\
\text { protein }^{2}\end{array}$ & $\begin{array}{l}\text { Fulyani et al. } 2013 \\
\text { (156) }\end{array}$ \\
\hline kupA* & Potassium transporter $^{2}$ & This work \\
\hline kupB* & Potassium transporter $^{2}$ & This work \\
\hline lysP* & Lysine specific permease $^{1}$ & Bolotin et al. 2011 \\
\hline lys $Q^{*}$ & Lysine specific permease $^{1}$ & Bolotin et al. 2011 \\
\hline $\operatorname{mvaA}$ & 3-hydroxy-3-methylglutaryl coenzyme A reductase ${ }^{1}$ & Bolotin et al. 2011 \\
\hline$n a h^{*}$ & $\mathrm{Na}^{+} / \mathrm{H}^{+}$antiporter ${ }^{1}$ & Bolotin et al. 2011 \\
\hline oppA* & Oligopeptide-binding protein ${ }^{1}$ & Bolotin et al. 2011 \\
\hline$p d p$ & Pyrimidine-nucleoside phosphorylase ${ }^{1}$ & Bolotin et al. 2011 \\
\hline $\operatorname{prfB}$ & Peptide chain release factor $2^{1}$ & Bolotin et al. 2011 \\
\hline $\operatorname{racD}$ & Aspartate racemase $^{1}$ & Bolotin et al. 2011 \\
\hline recG & ATP-dependent DNA helicase ${ }^{1}$ & Bolotin et al. 2011 \\
\hline recJ & Single-stranded DNA specific exonuclease ${ }^{1}$ & Bolotin et al. 2011 \\
\hline $\operatorname{rec} N$ & DNA repair protein $\mathrm{RecN}^{1}$ & Bolotin et al. 2011 \\
\hline relA & ppGpp synthetase $I^{1}$ & Bolotin et al. 2011 \\
\hline ribH & 6,7-dimethyl-8-ribityllumazine synthase ${ }^{1}$ & Bolotin et al. 2011 \\
\hline rpsF & $30 \mathrm{~S}$ ribosomal protein $\mathrm{S}^{1}$ & Bolotin et al. 2011 \\
\hline yсін & Ribonuclease $\mathrm{J}^{1}$ & Bolotin et al. 2011 \\
\hline yeaD & Initiation-control protein $\mathrm{YabA}^{1}$ & Bolotin et al. 2011 \\
\hline$y h f C$ & Uncharacterized protein & Bolotin et al. 2011 \\
\hline yjdJ* & Potassium channel protein ${ }^{1}$ & Bolotin et al. 2011 \\
\hline$y n c B^{*}$ & Uncharacterized protein & Bolotin et al. 2011 \\
\hline yqaB & Uncharacterized protein & Bolotin et al. 2011 \\
\hline yqaD & Uncharacterized protein & Bolotin et al. 2011 \\
\hline$y r b D^{*}$ & Uncharacterized protein & Bolotin et al. 2011 \\
\hline yrgI & Uncharacterized protein & Bolotin et al. 2011 \\
\hline yrjB & Oxidoreductase $^{1}$ & Bolotin et al. 2011 \\
\hline$y t c E$ & Uncharacterized protein & Bolotin et al. 2011 \\
\hline$y t j A$ & Uncharacterized protein & Bolotin et al. 2011 \\
\hline yueF & Protease $^{1}$ & Bolotin et al. 2011 \\
\hline
\end{tabular}


${ }^{1}$ Proteins inferred from homology. ${ }^{2}$ Experimental evidence available. *Genes selected independently from the pulldown experiment

\subsection{2. c-di-AMP specific interaction to different Lactococcal proteins}

Unexpectedly, the majority of the genes selected proved to have no specific interaction with c-di-AMP through the DrACALA assay. Nevertheless, two of the putative $\mathrm{K}^{+}$related proteins threw positive results. The corresponding genes were $k u p A$ and $k u p B$ and their products interact specifically with c-di-AMP according to the competition DrACALA experiment (Fig. 7). In addition, the interaction with KupA seems to be stronger than with KupB.

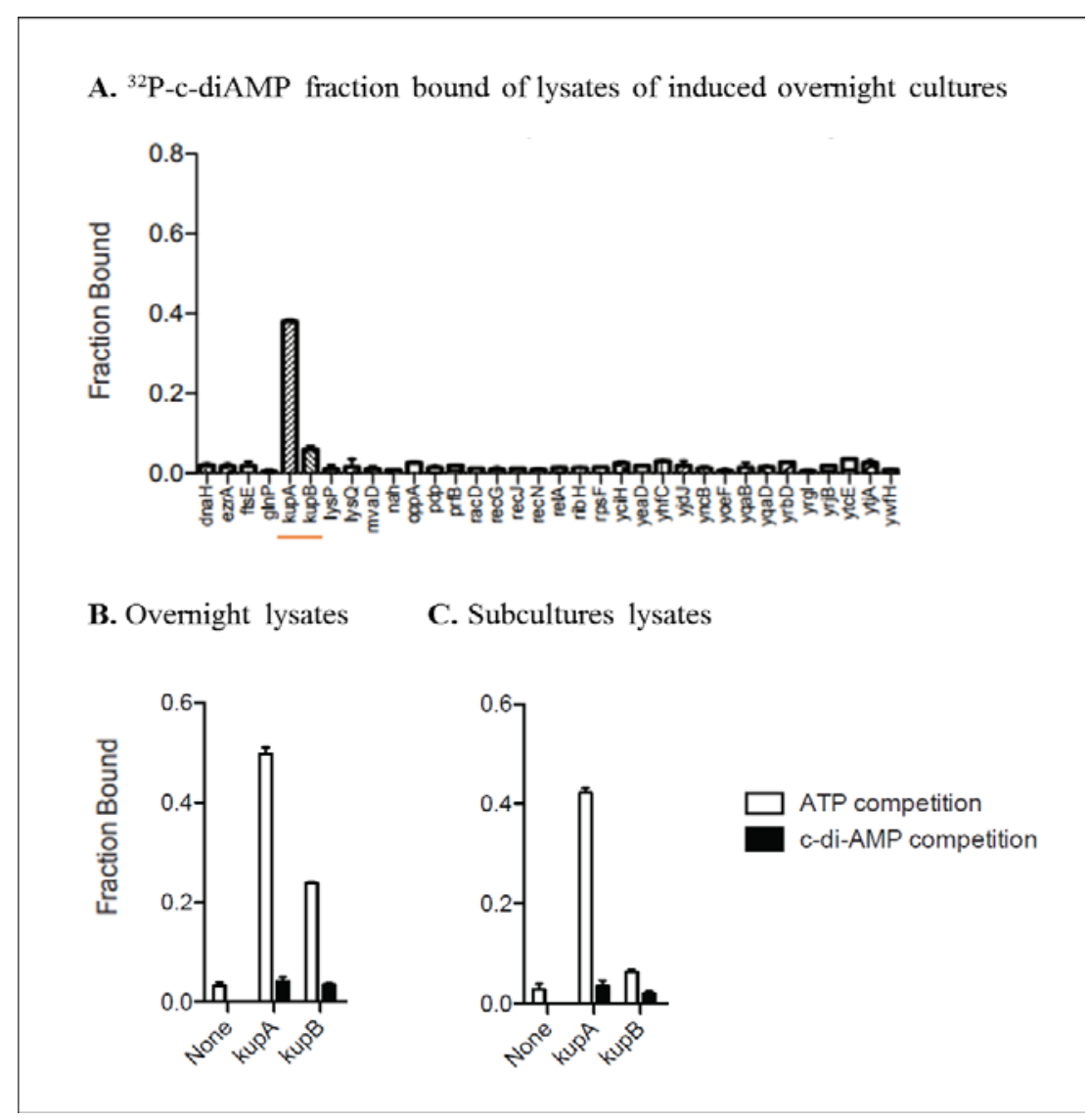

Fig. 7 DrACALA experiment. A: Fraction of radiolabeled c-di-AMP retained in induced overnight cultures. KupA and KupB specifically interact to c-diAMP (underlined in orange). ATP competition does not interfere with c-diAMP interaction in overnight (B) or subcultures lysates (C). Fresh c-di-AMP competition results in no ${ }^{32} \mathrm{P}$-c-di-AMP detected, indicating specific interaction. Induction of all samples was checked in overnight and midlog cultures (not shown).

Because of the lack of previously reported potassium transporters in L. lactis IL1403, a bioinformatic search was first performed. An initial approach showed that Kup proteins are 
widely found in bacteria as well as in species of fungi and plants. Nevertheless, the only reported investigation related to a Kup protein in L. lactis dates from 1997 when Quintero and Blatt analyzed a new transporters family in Arabidopsis and they see that it is conserved among different filos, since homologous sequences were found in different plant species, in L. lactis and also in Homo sapiens (157). However, up to date no other information can be found, which is why an in silico analysis of KupA and KupB was carried out.

In the model organism E. coli, Kup acts as a potassium intake system with particular characteristics that distinguish it from the potassium transporter families Ktr and Kdp, previously described. It belongs to the Kup/HAK/KT family and, as Fig. 8 represents, it is a 622 residues protein with 12 transmembrane fragments at the $\mathrm{N}$-terminal end (158).

A first study of the nucleotidic sequence of $L$. lactis IL1403 genome shows two adjacent genes of 2013 and 2016 base pairs, corresponding to kupA and kupB respectively, separated by 223 bp (Fig. 8, I). The amino acid sequence alignment between them shows a 73\% identity, whereas topology prediction using the free software Protter threw very similar structures with 12 transmembrane segments for both proteins under study, which correlates with E. coli Kup protein. Moreover, as Fig. 8 II shows, the majority of conserved residues are located in the Nterminus and particularly in the transmembrane regions. This makes sense provided E. coli does not synthesize c-di-AMP and, consequently, its Kup protein does not need to sense it. It is hence likely that in L. lactis the sensory domain has evolved from a common ancestor in the cytoplasmic C-terminal region, to acquire the capacity of c-di-AMP sensing and responding accordingly, modifying $\mathrm{K}^{+}$intake activity.

On the other hand, and even though the presence of acidic amino acids has been shown to be important for potassium transport, in Kup they are found in the transmembrane fragments of the protein. This has been reported as a unique feature of the Kup/HAK/KT family, not occurring neither for transporters of the Trk/Ktr/HKT nor for the Kdp family (158). Moreover, it was also proved that E. coli Kup residues D23, E116, E229 and D408 have a crucial role for Kup activity and its substitution for alanines resulted in a peptide that could not complement mutations in E. coli LB2003 to allow growth at low $\mathrm{K}^{+}$concentrations (see below). Interestingly, D23 and E229 are conserved in both Kup proteins of L. lactis IL1403 and despite E116 and D408 are not, in both KupA and KupB an aspartic acid and a glutamic acid residue are found instead respectively, so conservation of acidic residues remains (Fig. 8). 
On the other hand, sequence alignment of well-studied c-di-AMP binding potassium transporters shows a conserved glycine region, characteristic of Ktr family proteins. This GXGXXG consensus motif, typically present for nucleotide binding is not exactly conserved in Kup proteins but a GXXGXG inverted domain is found instead in both KupA and KupB (Fig. 8, II). This domain could be involved in c-di-AMP binding or could also suggest that Kup transporters need a cofactor like NAD for their activity (159).

Taken together, the evidence here presented supports the fact that proteins under study belong to the Kup/HAK/KT family but whether they are both active potassium transporters needed to be further studied.

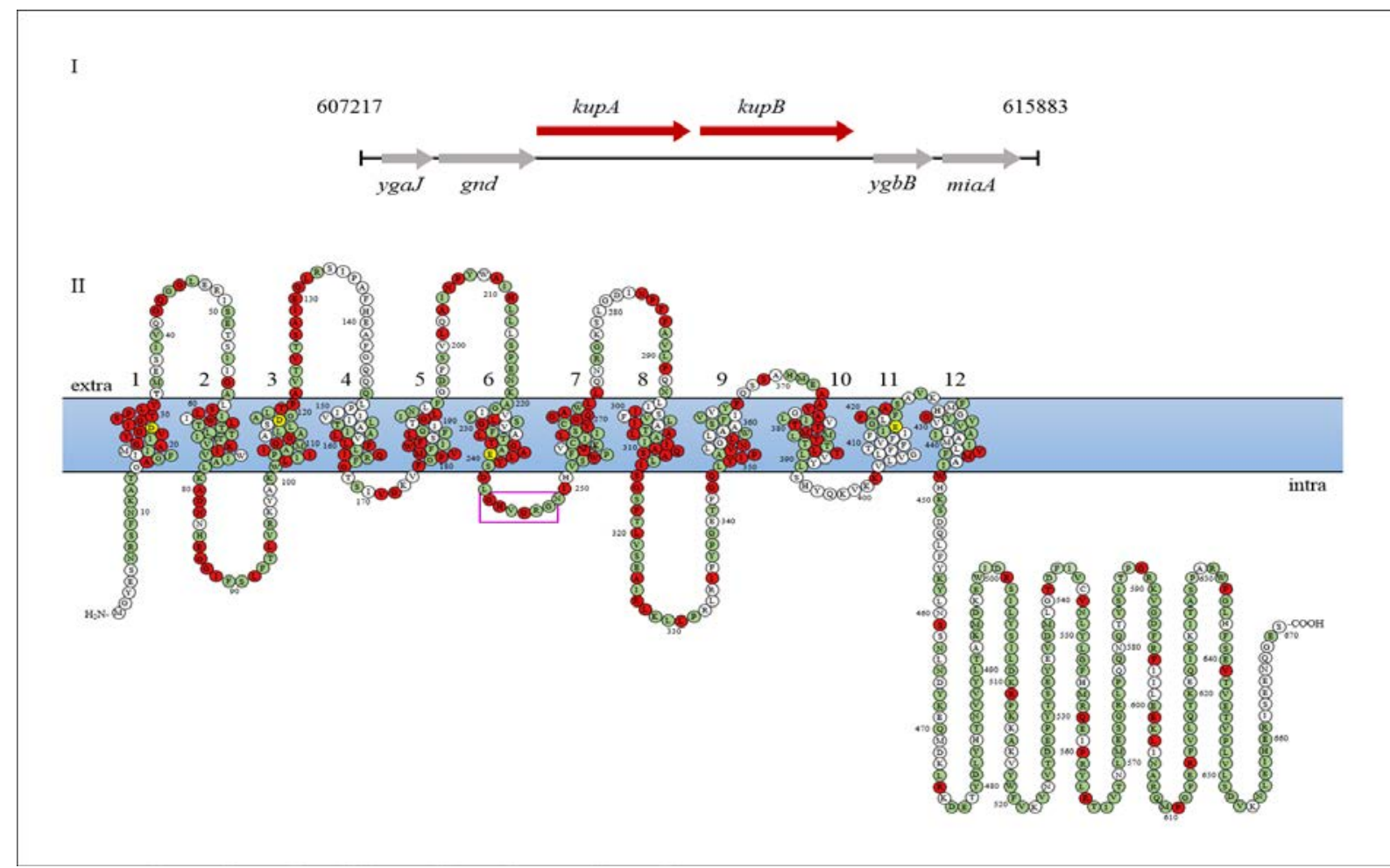

Fig. 8 KupA and KupB in silico analysis. I: Gene arrangement in L. lactis IL1403 genome. kupA and kupB are 2013 and 2016 base pairs genes respectively and are adjacently encoded. II: Predicted membrane conformation of KupA. Adapted from the free topology prediction Protter software. Amino acids conserved in both Kup proteins from L. lactis are highlighted in green, whereas those conserved in E. coli as well are represented in red. Conserved acidic amino acids are depicted in yellow. The cellular membrane is shown in light blue. Extra: extracellular, intra: intracellular. The purple box indicate a putative GXXGXG c-di-AMP binding site.

\subsubsection{KupA and KupB of $L$. lactis 1403 restore growth of $E$. coli LB650}

Heterologous expression of proteins in the model bacterium E. coli has always been one of the first choices when studying unknown protein functions. Consequently, a first approach into the functional properties of the proteins encoded by both kup genes was explored using $E$. 
coli LB650 (160). This strain is a triple mutant for the main potassium transporter systems, having a $\Delta k d p A B C 5 \Delta \operatorname{trk} H \Delta \operatorname{trk} G$ genotype (see Table XV). Hence, it is unable to grow at low $\mathrm{K}^{+}$concentrations.

kupA and kupB genes were respectively cloned in a modified version of plasmid pWH844, lacking the N-terminal His6x-tag after digestion with EcoRI restriction enzyme, which site is located upstream the His6x -tag codifying sequence. For this, primers IQ682 and IQ683 were used, which introduce an EcoRI restriction site as well as ribosome binding site. In this way, any functional interference produced by the histidine residues in the proteins was ruled out. The resulting plasmids pIQ309 and pIQ310, (carrying kupA and kupB genes, see Table XVI), were electroporated in E. coli LB650, originating strains LB09 and LB10 respectively, to check if their expression could restore growth in rich media when no $\mathrm{KCl}$ is added. As Fig. 9 shows, the strain harboring pWH844 vector cannot grow in LB, unless it is supplemented with $200 \mathrm{mM} \mathrm{KCl}$. On the other hand, when a trans copy of kupA or kupB is present, colonies appear on the plates without extra addition of $\mathrm{KCl}$, even when IPTG is not present. As mentioned in Material and Methods, pWH844 vector used for these constructions has the multiple cloning site under control of the lactose operon promoter (Plac), which is widely known to be leaky. Consequently, this basal expression of kupA and kupB is already enough to allow growth. These results show not only that the proteins under study are potassium transporters but also suggest that both transporters have high affinity for $\mathrm{K}^{+}$.

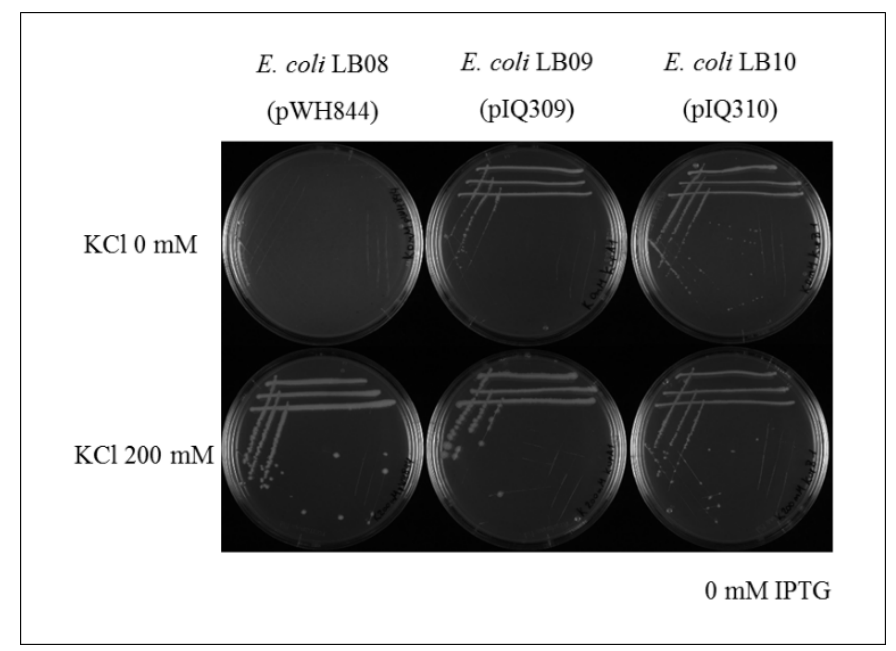

Fig. 9 KupA and KupB restore growth of $E$. coli LB650 in LB. Left: strain LB08 carrying pWH884 vector grows only when $200 \mathrm{mM} \mathrm{KCl}$ is added to the medium (down). Center and right strains LB09 and LB10 carrying a trans copy of $k u p A$ and $k u p B$, respectively. Both evidence growth with or without potassium supplementation. 


\subsubsection{KupA and KupB are high affinity transporters}

In order to estimate the affinity of KupA and KupB proteins for $\mathrm{K}^{+}$, a comparison to previously characterized transporters was made. For this, the high affinity potassium transporter KtrA/B from B. subtilis and the low affinity KtrC/D system from L. monocytogenes were employed $(48,161)$. The corresponding genes had been previously cloned in the modified version of pWH844 plasmid described in the former section, lacking the His6x-tag. The resulting plasmids pBP372 and pBP371 were available at Prof. Stülke’s lab and were electroporated in strain E. coli LB650, originating strains LB11 and LB12, respectively.

Growth curves were then performed in minimal salt media M9 modified, where potassium salts were replaced by equimolar quantities of the respective sodium salt (M9mod, see Materials and Methods). $\mathrm{KCl}$ was then added to the media, to reach final concentrations between $0.025-50.0 \mathrm{mM}$, and determine in this way the concentration at which growth was possible. OD $\max$ and $\mu_{\max }$ were then determined for strains E. coli LB08, LB09 and LB10, harboring the empty vector, and kupA and kupB genes, respectively. Strains LB11 and LB12 were included as reference of the response of a high and a low affinity system under the conditions here studied.

Both Kup proteins showed similar phenotypes, manifesting growth even at concentrations below $0.1 \mathrm{mM} \mathrm{KCl}$, although growth rate and final OD600 were low (Fig. 10 and Table XIII) Moreover, at $1.0 \mathrm{mM} \mathrm{KCl}$, values close to the highest $\mathrm{OD}_{\max }$ and $\mu_{\max }$ are already reached, and coincide with values obtained for the high affinity system KtrA/B of B. subtilis. On the other hand, for the case of $\mathrm{KtrC} / \mathrm{D}$, growth is only possible at $10 \mathrm{mM} \mathrm{K} \mathrm{K}^{+}$or higher, and maximum ODmax and $\mu_{\max }$ values are obtained when at least $50 \mathrm{mM} \mathrm{K}^{+}$is present in the media. A marked difference is then established with respect to high affinity KtrA/B and Kup proteins, being more closely related to strain LB08 harboring the empty vector. Taken together, these results support the theory that both Kup proteins are high affinity potassium transporters. 


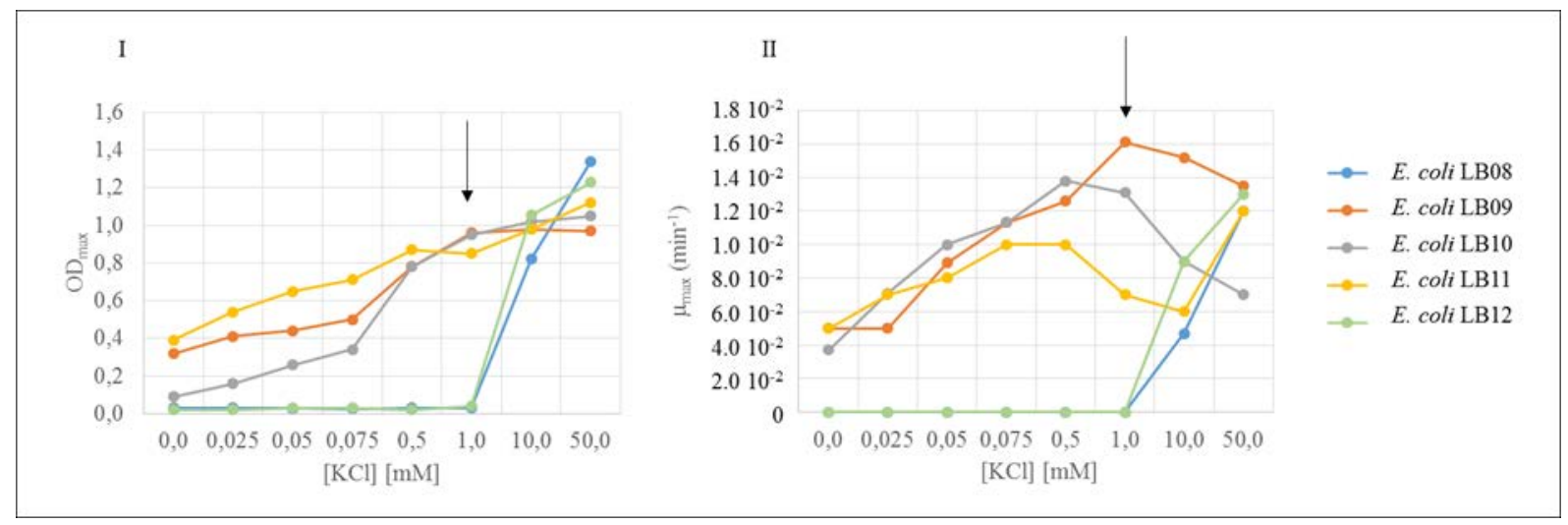

Fig. 10 Growth parameters of $\boldsymbol{E}$. coli LB650 derived strains. Growth curves were performed in a microplate reader and in minimal salt $\mathrm{M} 9$ mod medium. $\mathrm{OD}_{\max }$ (I) and $\mu_{\max }$ (II) were determined at different $\mathrm{KCl}$ concentrations. The arrow highlights parameters obtained at $1.0 \mathrm{mM} \mathrm{KCl}$, where the different potassium transporter systems show clear distinct phenotypes. Both Kup proteins behaved similarly to the high affinity KtrA/B system.

Table XIII. $\mathrm{OD}_{\max }$ and $\mu_{\max }$ values for E. coli LB650 derivative strains

\begin{tabular}{|c|c|c|c|c|c|c|c|c|c|c|}
\hline & \multicolumn{2}{|c|}{ pWH844 } & \multicolumn{2}{|c|}{ KupA } & \multicolumn{2}{|c|}{ KupB } & \multicolumn{2}{|c|}{ KtrA/B } & \multicolumn{2}{|c|}{$\mathrm{KtrC} / \mathrm{D}$} \\
\hline$\left[\mathrm{K}^{+}\right][\mathrm{mM}]$ & $\mathrm{OD}_{\max }$ & $\mu_{\max }$ & $\mathrm{OD}_{\max }$ & $\mu_{\max }$ & $\mathrm{OD}_{\max }$ & $\mu_{\max }$ & $\mathrm{OD}_{\max }$ & $\mu_{\max }$ & $\mathrm{OD}_{\max }$ & $\mu_{\max }$ \\
\hline- & ND & ND & 0.3 & $5.0 \mathrm{E}-3$ & 0.1 & $3.7 \mathrm{E}-3$ & 0.4 & $5.0 \mathrm{E}-3$ & ND & ND \\
\hline 0.025 & ND & ND & 0.4 & $5.0 \mathrm{E}-3$ & 0.2 & $7.1 \mathrm{E}-3$ & 0.5 & $7.0 \mathrm{E}-3$ & ND & ND \\
\hline 0.50 & ND & ND & 0.4 & 8.9E-3 & 0.3 & $1.0 \mathrm{E}-2$ & 0.7 & $8.0 \mathrm{E}-3$ & ND & ND \\
\hline 0.075 & ND & ND & 0.5 & $1.1 \mathrm{E}-2$ & 0.3 & $1.1 \mathrm{E}-2$ & 0.7 & $1.0 \mathrm{E}-2$ & ND & ND \\
\hline 0.50 & ND & ND & 0.8 & 1.3E-2 & 0.8 & $1.4 \mathrm{E}-2$ & 0.9 & $1.0 \mathrm{E}-2$ & ND & ND \\
\hline 1.0 & ND & $\mathrm{ND}$ & 1.0 & $1.6 \mathrm{E}-2$ & 1.0 & $1.3 \mathrm{E}-2$ & 0.9 & $7.0 \mathrm{E}-3$ & $\mathrm{ND}$ & ND \\
\hline 10.0 & 0.8 & $4.7 \mathrm{E}-3$ & 1.0 & $1.5 \mathrm{E}-3$ & 1.0 & $9.0 \mathrm{E}-3$ & 1.0 & $6.0 \mathrm{E}-3$ & 1.1 & $9.0 \mathrm{E}-3$ \\
\hline 50.0 & 1.3 & $1.2 \mathrm{E}-2$ & 1.0 & $1.6 \mathrm{E}-2$ & 1.1 & $7.0 \mathrm{E}-3$ & 1.1 & $1.2 \mathrm{E}-2$ & 1.2 & $1.3 \mathrm{E}-2$ \\
\hline
\end{tabular}

\subsection{5. c-di-AMP down-regulates Kup proteins}

Once specific interaction with c-di-AMP was confirmed, and KupA and KupB were identified as potassium transporters, the next step was to analyze the impact of this metabolite on the activity of both Kup proteins. To this aim, a co-expression system was established in the model bacterium E. coli 2003. This strain is deficient in the three major potassium uptake systems Trk, Kup and Kdp ( $\triangle k d p A B C 5 k u p D 1 \Delta t r k A$, see Table XV), and is not able to grow in minimal salt media at low $\mathrm{K}^{+}$concentrations without a trans complementation of a potassium transporter coding gene. Very importantly, and in contrast to strain LB650 $\left(\mathrm{Km}^{50}-\mathrm{Cm}^{30}\right)$, its mutations are clean, so there is no need for antibiotic supplementation of growth media. Moreover, E. coli lacks c-di-AMP synthesizing enzymes, for which the co-expression of $c d a A$ and kup genes allows the analysis of the phenotypic effect of c-di-AMP on Kup proteins, without interference of host-synthesized c-di-AMP. 
At the beginning of this thesis, several attempts were made to heterologously produce CdaA enzyme from L. lactis IL1403 in E. coli. Since no successful results were obtained, and taking into account that for the practical scope of the experiment only the production of c-diAMP was necessary, independently of the synthesizing enzyme, CdaA from $L$. monocytogenes (from now on $\mathrm{CdaA}^{\mathrm{Imo}}$ ) was used instead. A non-active defective version of it, $\mathrm{CdaA}^{\text {lmo*}}$, was used as well, as negative control.

Plasmids pIQ309 and pIQ310 (Amp ${ }^{100}$ ) were used for kup genes induction via IPTG, whereas $c d a A^{\text {Imo }}$ and $c d a A^{\text {Imo* }}$ were induced from plasmids pBP370 and pBP373 respectively (kindly supplied by Prof. Stülke laboratory). These plasmids are pBAD33 derivatives $\left(\mathrm{Cm}^{30}\right.$, see Table XVI), allowing expression of genes under control of the arabinose Para promoter, and being therefore compatible with pWH844 derivatives previously mentioned. A system of co-expression was then established where potassium transporters could be induced by IPTG and c-di-AMP cyclases by arabinose.

In the previous section, where growth experiments were performed in E. coli LB650 harboring a trans copy of kupA and kupB, addition of $0.1 \mathrm{mM} \mathrm{KCl}$ to $\mathrm{M} 9 \mathrm{mod}$ medium was enough for the strains under study to grow. Hence, this concentration was selected to supplement M9mod medium for the growth curves performed on the E. coli 2003 derivative strains. It is important to mention that under this potassium concentration, strain E. coli 0380, harboring vectors pWH844 and pBP370 $\left(c d a A^{l m o}\right)$, is not able to grow, which is why it was used as negative control.

As seen in Fig 11, IPTG induction of kupA gene allowed growth of strains E. coli 0390 and 0393. Nevertheless, when $c d a A^{l m o}$ (present in strain 0390) is also induced via arabinose supplementation in growth media, a severe growth detriment is evidenced (Fig 11, left). On the other hand, either when the inductor (arabinose) is not present (Fig 11, right), or when $c d a A$ gene carries a mutation that results in an inactive enzyme (strain 0393), growth is possible again. 


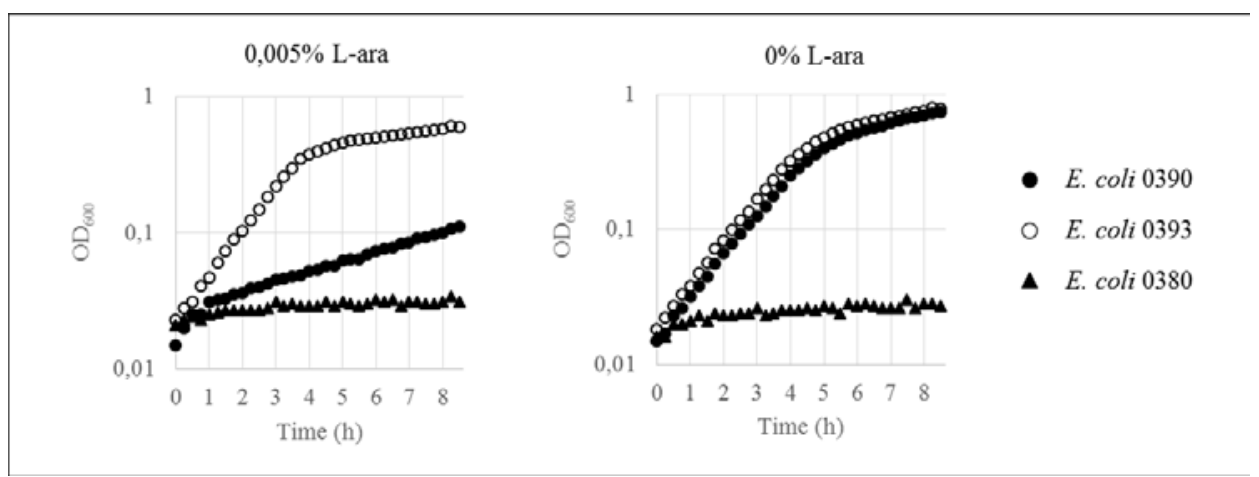

Fig 11 c-di-AMP effect on KupA. Growth curves were performed in minimal salt M9mod media, supplemented with $2.5 \mu \mathrm{M}$ IPTG for kupA induction and with $0.005 \% \mathrm{~L}$ arabinose (left) or without it (right). Strain 0390 and 0393 harbor a copy of $c d a A^{\text {lmo }}$ and $c d a A^{\text {lmo* }}$, respectively. Both carry as well a copy of kupA. E. coli 0380 with the empty vector pWH844 and pBP370 was used as negative control.

The same experiment was performed to study the impact of c-di-AMP on KupB. In this case, results obtained were similar, but the growth inhibition exerted on strain 0300 (harboring plasmids pIQ310 with a copy of $k u p B$ and pBP370, with wild type $c d a A^{\text {Imo }}$ ) in induced conditions, was milder (Fig 12, left).

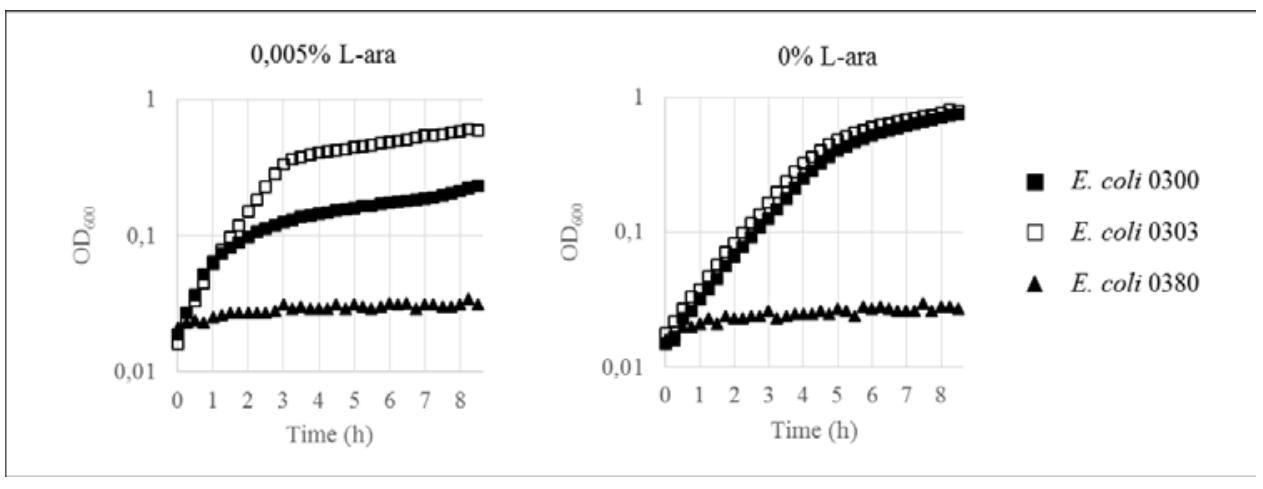

Fig 12 c-di-AMP effect on KupB Growth curves were performed in minimal salt M9mod media, supplemented with $2.5 \mu \mathrm{M}$ IPTG for kupB induction and with $0.005 \% \mathrm{~L}$-arabinose

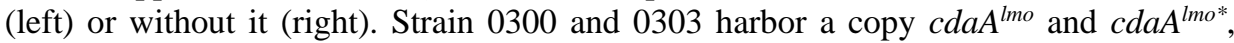
respectively. Both carry as well a copy of kupB. E. coli 0380 with the empty vectors pWH844 and pBP370 was used as negative control.

It is important to mention that the growth inhibition observed in both cases could be caused by high intracellular c-di-AMP concentrations, which have been reported to be toxic for bacteria (54). On the other hand, it could also be due to the production of the heterologous $\mathrm{CdaA}^{\mathrm{lmo}}$ enzyme, which could be toxic for the cell independently of c-di-AMP concentration or the presence of the potassium transporters under study. This was ruled out by performing similar growth curves of $E$. coli 0380 harboring an empty copy of vector pWH844 and plasmid pBP370. This time, $50 \mathrm{mM} \mathrm{KCl}$ was added to growth media, and wild type growth was possible 
under arabinose induction as well as no-induction conditions (Fig 13). As expected, strain 0383 harboring the mutated $c d a A^{l m o^{*}}$, was not inhibited either and both strains evidenced similar growth phenotypes to strain 4433, with both empty vectors pWH844 and pBAD33. Altogether, the evidence here presented confirms that c-di-AMP downregulates KupA and KupB proteins.

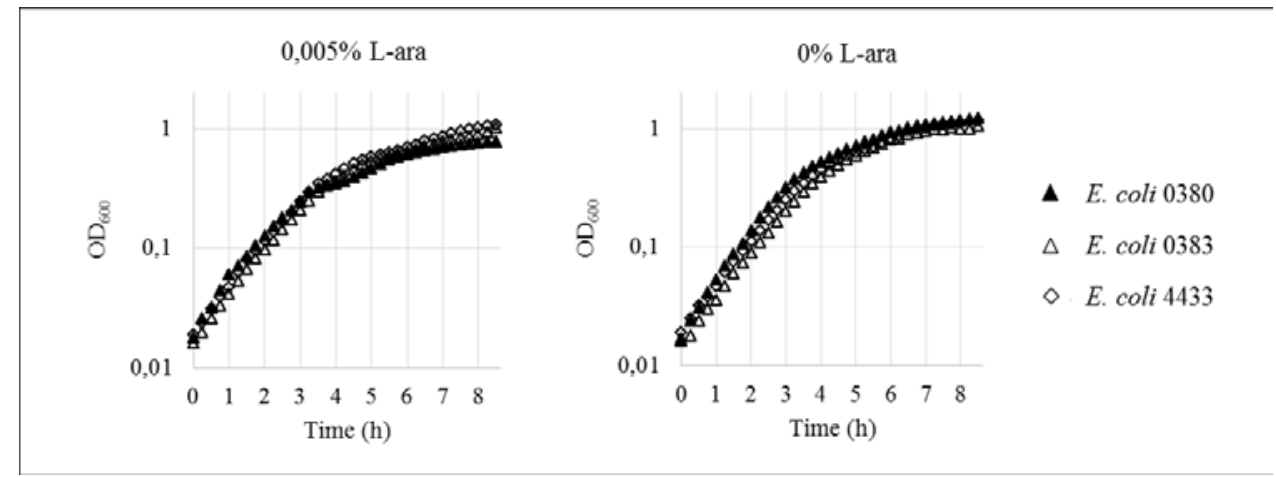

Fig 13 Control curves on M9mod supplemented with $50 \mathrm{mM}$ KCl. High potassium concentrations allowed similar growth under arabinose induction (left) and no-induction conditions (right). 0380: pWH844 plus pBP370 (cdaAlmo), 0383: pWH844 plus pBP373 (cdaAlmo*), 4433: pWH844 plus pBAD33.

\subsubsection{Conclusions}

In this chapter, a pull-down experiment with strep-tagged magnetic beads coupled to biotinilated c-di-AMP was performed in order to identify novel interaction partners of this second messenger in L. lactis IL1403. Even though no specific binging protein was recovered from this experiment, the DrACALA assay permitted the identification of two novel potassium transporters, which specifically interact with c-di-AMP: KupA and KupB. Minimal KCl concentrations restoring growth of mutant strains E. coli LB650 and 2003 in M9mod medium, as well as comparisons to previously studied high and low affinity systems (B. subtilis KtrA/B and L. monocytogenes KtrC/D, respectively) provide evidence that, unlike in E. coli, L. lactis Kup proteins are high affinity potassium transporters.

An in silico analysis showed that KupA and KupB share $73 \%$ identity and when compared to E. coli Kup, the most conserved regions are in the transmembrane fragments, whereas the major variability occurs in the cytosolic C-terminal end. Moreover, after sequence analysis of other c-di-AMP binding proteins, a putative c-di-AMP binding domain was hypothesized.

Finally, a co-expression system was constructed, allowing controlled induction of kup genes under study and $c d a A$, by use of an IPTG and an arabinose inducible promoter, 
respectively. Growth curves performed under low potassium concentrations confirmed that cdi-AMP has an inhibitory effect on both KupA and KupB. 


\subsection{Studies on c-di-AMP synthesizing and degrading enzymes in $L$. lactis IL1403}

\subsubsection{Strain engineering for modification of intracellular c-di-AMP levels}

The first approach aiming to modify intracellular levels of c-di-AMP in L. lactis was directed towards deletion of the gene encoding its only synthesizing enzyme, cdaA. Several attempts were made by use of vector pBVGh, previously developed in our laboratory and described below. Since deletion in rich medium M17G was not possible, and mutants for c-diAMP cyclases were reported on strictly controlled minimal media, the deletion protocol was tuned to be performed in defined media. As previously described, lactic acid bacteria lack many anabolic pathways and, consequently, a considerable amount of nutrients have to be provided in growth media, in order to satisfy their complex metabolic requirements. Different conditions were thus tried, to establish a suitable defined medium, which allowed regulation of potassium concentration and, at the same time, high L. lactis cellular density.

Minimal medium MS15 was employed as described by Cocaign-Bousquet et al. (162) and using the same criteria as for M9 minimal salt medium in the previous chapter, potassium salts were replaced by equimolar quantities of the corresponding sodium salt, resulting in MS15m medium. Different KCl concentrations were then used to supplement MS15m and determine the minimum potassium molarity allowing an OD600 high enough to perform the different steps of the deletion protocol. Fig 14, I shows the final OD600 obtained for L. lactis IL1403 and NZ9000 strains after 5 days of incubation at 30 ${ }^{\circ}$, when $1.0-5.0-10-100$ and $200 \mathrm{mM} \mathrm{KCl}$ were added to MS15m. Since the original protocol requires several ON incubation steps, use of this medium would be practically unviable.

On the other hand, Zhang et al developed another minimal medium, which permitted high density growth of lactococci (163). This medium, called ZMB2 was also modified to avoid use of potassium salts in its initial composition, resulting in ZMB2m medium. This time, optical densities above 1.0 were obtained for $\mathrm{KCl}$ concentrations between 1.0 and $100 \mathrm{mM}$ in $\mathrm{ON}$ cultures of both strains under study (Fig 14, II and III). Hence, ZMB2m medium and KCl concentrations of $0.25,0.50$ and $1 \mathrm{mM}$ were selected to perform the deletion protocol. 


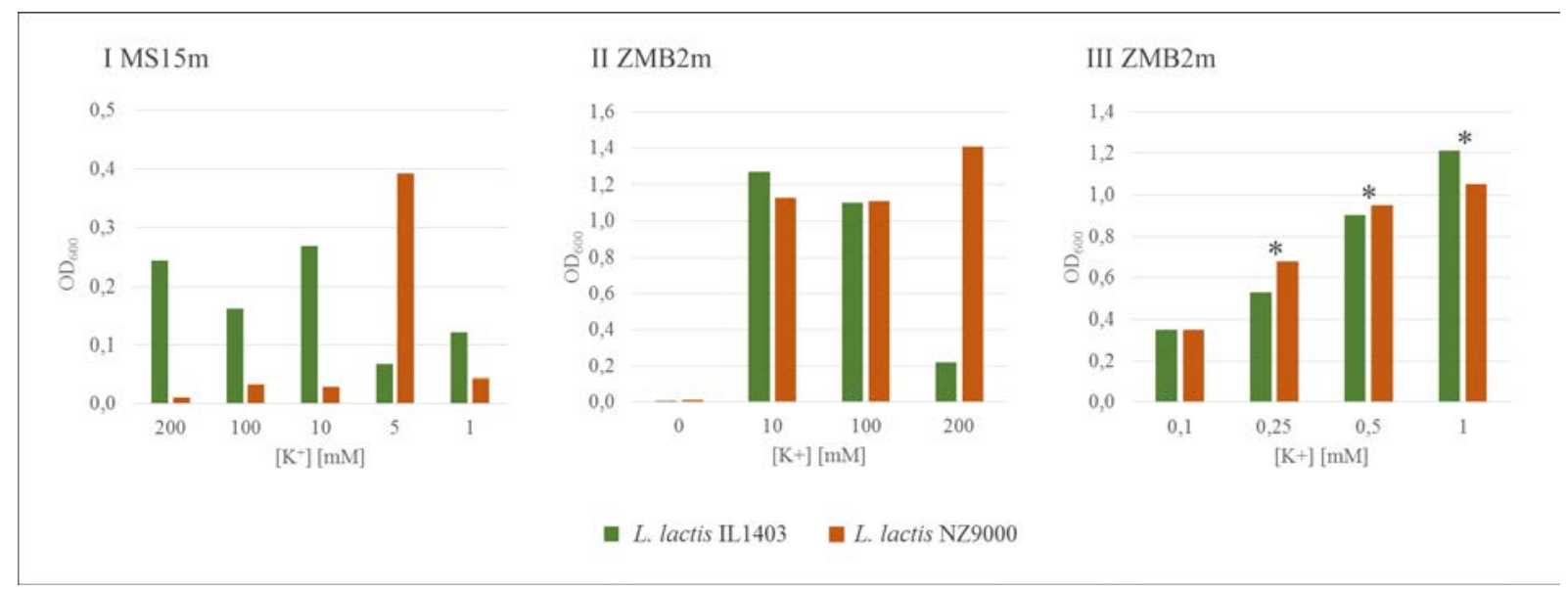

Fig 14 L. lactis growth on minimal media. Strains IL1403 and NZ9000 were cultured in modified minimal media without potassium salts. $\mathrm{KCl}$ was added prior to inoculation at the concentrations detailed in the graphs. I: Final $\mathrm{OD}_{600}$ obtained in MS15m after 5 days. II and III: Final $\mathrm{OD}_{600}$ obtained in ZMB2m ON cultures. KCl concentrations used in the deletion protocol are marked with an asterisk.

Even though mutants lacking c-di-AMP synthesizing enzymes were reported to be obtained in minimal media at low $\mathrm{K}^{+}$concentrations $(49,60,62)$, cdaA deletion was not possible for neither of L. lactis strains under study in this work. A second approach was also tried, where by use of the nisin inducible pNZ8048 vector, genomic cdaA deletion was tried when a trans copy was being expressed. In this way, in the absence of nisin, cells would be depleted of CdaA enzymes, and mutants and possible suppressors could be studied. Unfortunately, this strategy was not successful either, for what the focus of this research was now directed towards the modification of c-di-AMP intracellular pools and the obtaining of maximum and minimum levels compatible with growth in rich media for L. lactis IL1403.

The starting point to this aim was the development of genetically engineered strains for homologous and heterologous expression of genes encoding c-di-AMP synthesizing and degrading enzymes. Thus, $c d a A$ and gdpP genes from L. lactis IL1403 and E. faecalis JH2-2 ( $c d a A^{l l}, g d p P^{l l}, c d a A^{e f}$ and $g d p P^{e f}$, respectively) were cloned in vector pBV153 (Fig 15, I). This vector was developed in our laboratory and it has the Pcit promoter upstream of the multiple cloning site, leaving the gene of interest under $\mathrm{pH}$ regulation (164). That is to say, as the $\mathrm{pH}$ of the growth medium decreases, expression of the desired protein increases. The resulting plasmids were named pQI101, pQI102, pQI103 and pQI104, respectively and were electroporated in L. lactis IL1403 originating strains LL03, LL04, LL05 and LL06, respectively (see Table XV). 


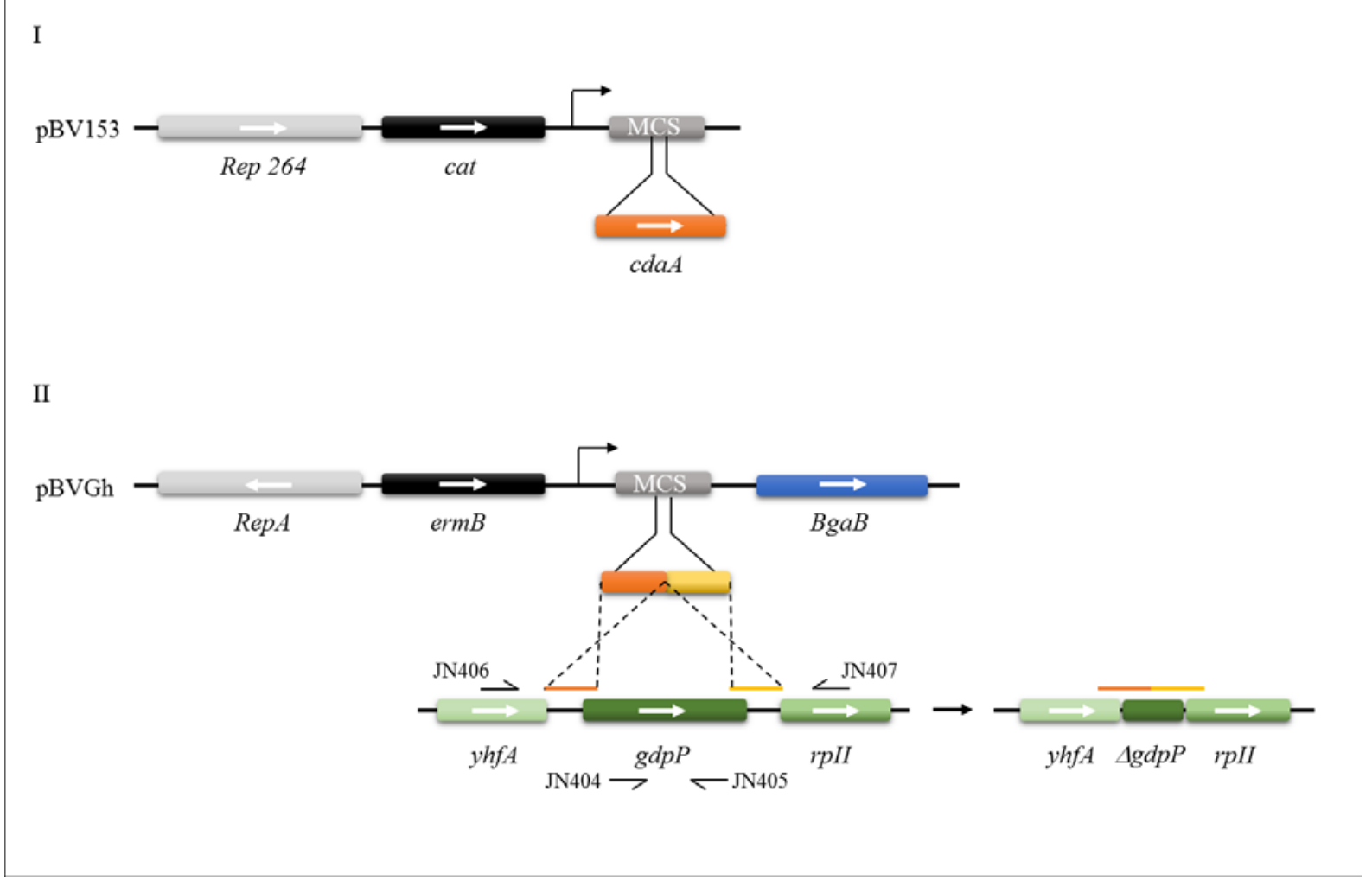

Fig 15 Strain development for modification of c-di-AMP intracellular levels. I: Vector pBV153 was used for homologous and heterologous expression of $c d a A^{l l}, g d p P^{l l}, c d a A^{e f}$ and $g d p P^{e f}$. Only $c d a A$ is represented, but the same system was used in a similar way to clone $g d p P$ genes as well. II: pBVGh vector employed for gene deletion. This system allows a clean deletion of the gene of interest via a double recombination event. Primers JN404 and JN405 as well as JN406 and JN407 were used for deletion check.

Another strategy considered for modifying intracellular c-di-AMP concentrations was mutation of its degrading enzyme, GdpP. Since this is the only c-di-AMP phosphodiesterase reported in L. lactis, deletion of its gene via homologous recombination by use of vector pBVGh was performed (Fig 15, II). The selected thermosensitive vector was previously developed in our laboratory as well, and it allows deletion of the gene of interest via a series of incubations at the permissive and non-permissive temperature, without incorporation of antibiotic resistances in the strain under study (165). Briefly, an up and a downstream region of the gene are adjacently cloned in the plasmid, so homology enables the integration of the plasmid in the chromosome when incubating at the non-permissive temperature. When temperature is lowered, the plasmid jumps out and it carries the gene of interest with it. An easy white/blue colony screen allows identification of putative mutants, which are then checked by antibiotic sensitivity and by two different PCR. The first one is performed with a pair of primers hybridizing outside the fragments cloned (JN406 and JN407 in Fig 15, II), and the second one is a negative control for which primers amplifying a central region of the gene of interest are used (JN404 and JN405 in Fig 15, II). 
Once deletion of $g d p P$ was confirmed, the selected strains were restricked three times to check colony morphology and growth stability. Interestingly, in this process, two slightly different colony sizes were obtained, for which both samples were kept for later c-di-AMP levels determination. These strains were named L. lactis LL07 (smaller colonies) and LL08 (bigger colonies).

\subsubsection{Phenotypic analysis of the strains developed}

The first analysis of strains constructed was performed on morphology developed in rich media M17G during exponential growth (Fig 16, up) and also once the stationary phase was reached (Fig 16, down). In the first case, the wild type phenotype can be described as short chains of two or four bacteria, with homogeneous and well-defined round shape. This is also true for strains LL05 and LL06, respectively carrying $g d p P^{l l}$ and $g d p P^{e f}$ genes, where no particular phenotype is observed. The main differences, on the other hand, were obtained for strains LL03 and LL04, harboring a trans copy of $c d a A^{l l}$ and $c d a A^{e f}$, respectively. In both these cases, a slight increase in cell size can be visualized, as well as no clear division of each cell in dimeric chains. As previously mentioned and as it is widely known, c-di-AMP metabolism is closely related to cell wall biosynthesis, which is why this phenotype would indicate that $c d a A$ genes are being expressed.

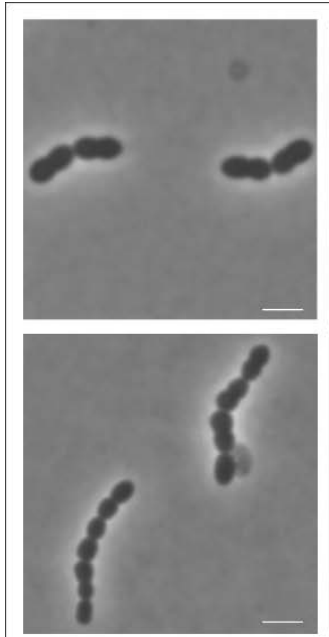

L. lactis IL1403 LL00

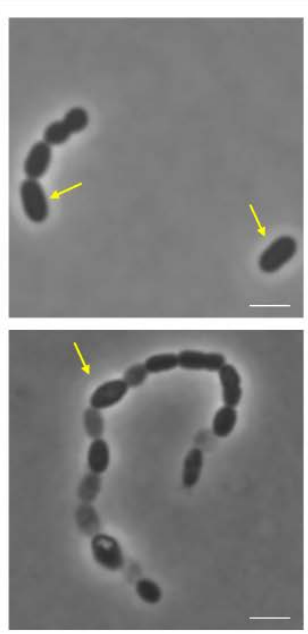

L. lactis IL1403 LL03

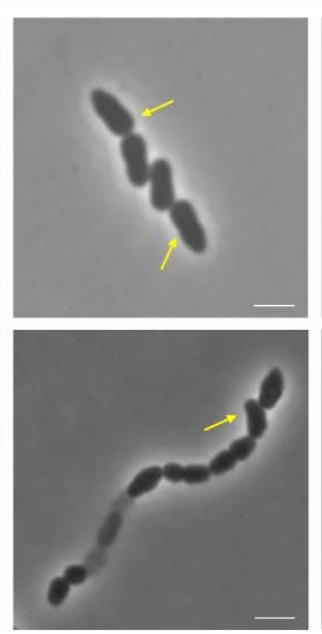

L. lactis IL1403 LL04

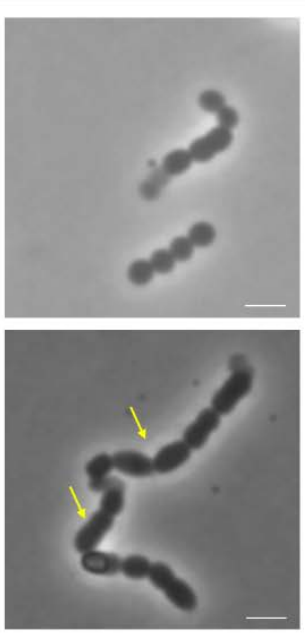

L. lactis IL1403 LL05

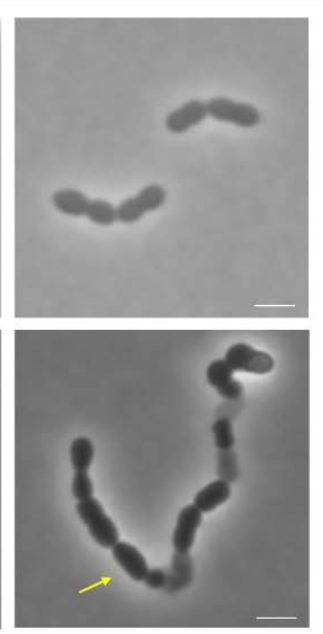

L. lactis IL1403 LL06

Fig 16 Morphology of $L$. lactis LL00, LL03-LL06 strains. Phase contrast microscopy of strains LL00, LL03LL06. Bacteria were grown in M17G medium at $30{ }^{\circ} \mathrm{C}$ and samples were taken at mid-exponential phase (up) and at stationary phase (down). Arrows indicate abnormal morphology. Scale bar, $2 \mu \mathrm{m}$. 
Clearer phenotypes were evidenced for the stationary phase, although they were similar for all strains under study. Control strain LL00 harboring the empty vector formed long cocci shaped strains, whereas strains LL03-LL6 evidenced heterogeneous bacterial sizes and shapes, as well as a higher number of non-viable cells. These results indicate that morphology is more affected in the late exponential phase, probably due to accumulation of c-di-AMP (for strains LL03 and LL04) or long time exposure to low levels of this second messenger (for strains LL05 and LL06).

After analyzing morphology of strains developed to alter c-di-AMP intracellular levels, a second study was done on growth curves obtained in rich media M17G upon addition of different stress factors. Although induction of Pcit promoter increases as the $\mathrm{pH}$ of the media decreases, these experiments were performed at initial $\mathrm{pH}$ of 7.0 to avoid raising c-di-AMP concentrations into toxic levels. Indeed, the capacity of LAB to acidify media as result of their lactic acid production was ideal to increase induction gradually along growth.

Control curves in $\mathrm{M} 17 \mathrm{G}$ grown at $30^{\circ} \mathrm{C}$ can be seen in Fig 17, where strain LL03 carrying $c d a A^{l l}$ gene, already shows a growth disadvantage with respect to strain LL00 harboring vector pBV153. This detriment is evidenced by a particularly long lag phase, which is enhanced when temperature is raised to $37^{\circ} \mathrm{C}$. Interestingly, Smith et al. reported heat resistant L. lactis strains obtained after construction of $g d p P$ mutants by use of a protocol which involved an incubation step at $37.5^{\circ} \mathrm{C}(74)$. Taking into account that a defective $g d p P$ strain and a strain with an extra copy of $c d a A$ would have the same effect of increasing c-di-AMP concentration, similar phenotypes were expected. Therefore, the growth defect manifested by strain LL03, could suggest that an even a higher c-di-AMP concentration was reached with respect to the gdpP mutants, resulting toxic for the cell, or a mutation in another gene involved in heat resistance.

Strains L. lactis LL05 and LL06 ( $g d p P^{l l}$ and $g d p P^{e f}$, respectively) also evidenced a growth disadvantage, although to a lesser extent than strain LL03.This time, results are in accordance with the heat resistance phenotype of $g d p P$ mutants, since an extra copy of the gene would decrease c-di-AMP intracellular pools, causing the opposite effect. Taken together, evidence here presented agree with previous findings about low as well as high concentrations of c-di-AMP being toxic for bacteria (54). Lastly, strain LL04 harboring a trans copy of $c d a A^{e f}$ gene seems to have no effect on growth, suggesting that it could be inactive in the system here studied. 

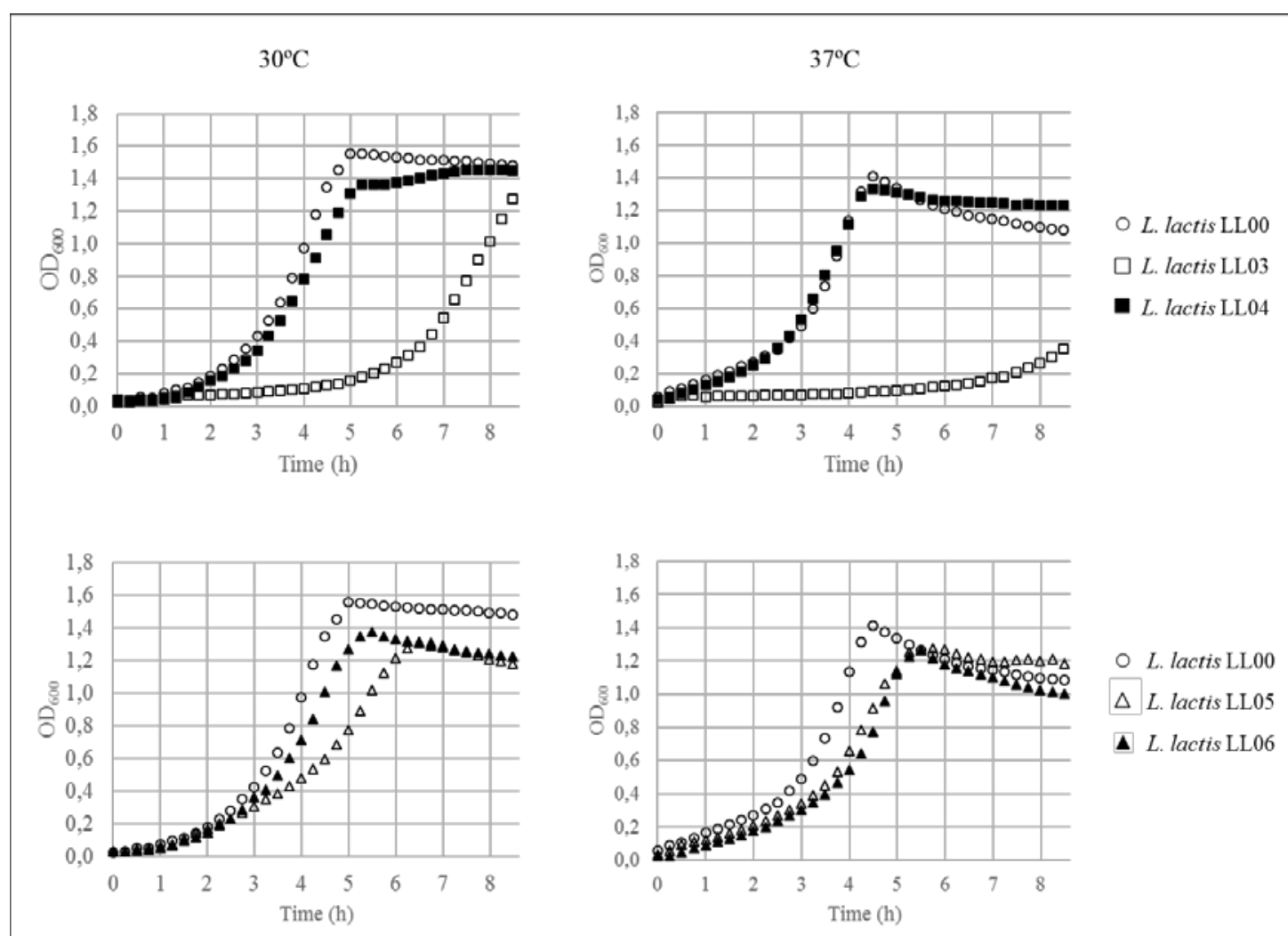

Fig 17 Temperature sensitivity of strains LL00, LL03 - LL06. Growth curves were performed in rich media M17G at $30{ }^{\circ} \mathrm{C}$ (left) and $37{ }^{\circ} \mathrm{C}$ (right). Strain L. lactis LL00 harbors the empty vector pBV153, whereas the series LL03, LL04, LL05 and LL06 carry a trans copy of genes $c d a A^{l l}, c d a A^{e f}$, $g d p P^{l l}$, and $g d p P^{e f}$, respectively.

Moreover, saline hypersensitivity was also reported by Smith et al. for gdpP mutant strains of L. lactis, and was therefore evaluated for the strains developed in this work (74). Concentrations of 0.25 and $0.50 \mathrm{M} \mathrm{NaCl}$ were selected to analyze their impact on growth. As Fig 18 shows, strain LL03 develops an unusually long lag phase of 8 hours and reaches stationary phase after 12 hours. Moreover, when media is supplemented with $\mathrm{NaCl} 0.50 \mathrm{M}$, a general detriment in growth is observed, since maximum OD600 is lowered, and strain LL03 is not able to grow anymore. On the other hand, strain LL05 (harboring a trans copy of $g d p P^{l l}$ ) also shows impaired growth, although the effect is not as marked as the one just described. Conversely, neither the presence of $c d a A^{e f}$ in strain LL04, nor of $g d p P^{e f}$ in strain LL06seems to have an evident impact on growth under these conditions, since in both cases growth is similar to strain LL00, carrying the empty vector.

Impact of $\beta$-lactam antibiotics on strains under study was also tested. These are a class of antibiotics active against a group of proteins called "penicillin-binding proteins” or PBP. A subgroup of essential PBP are the transpeptidases, which are serine proteases in charge of the last step in cell wall biosynthesis: the cross-linking of neighboring peptidoglycan strands (166). 
The $\beta$-lactam ring of these antibiotics mimics the cell wall strand, but once in contact with the enzyme, it forms a stable intermediate that inactivates the transpeptidase. Consequently, an imbalance occurs between the incorporation of new building blocks to the cell wall and the recycling activity of autolysins, leading to cell lysis (167).

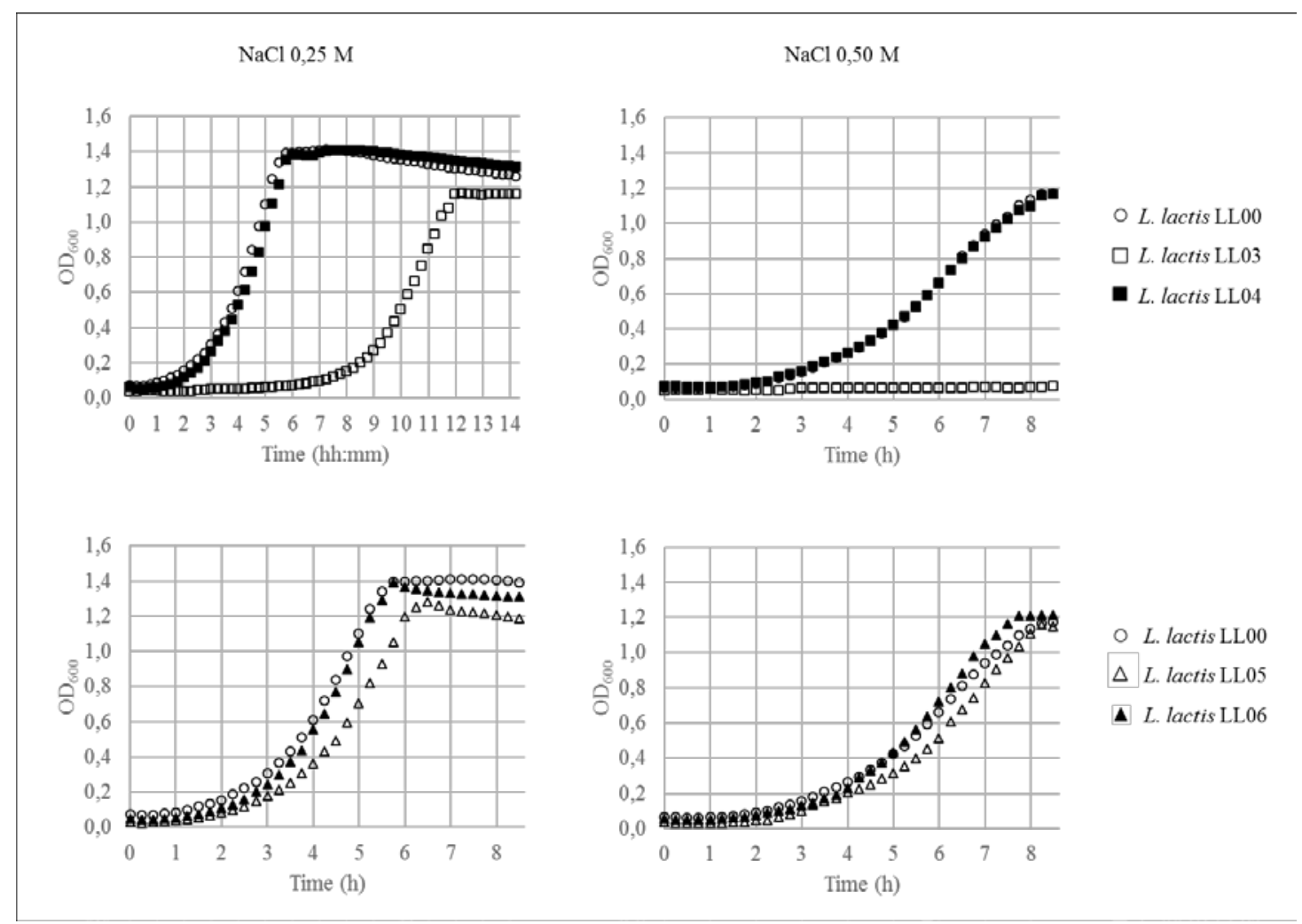

Fig 18 Saline sensitivity of strains LL00, LL03 - LL06. Growth curves were performed at $30{ }^{\circ} \mathrm{C}$ in rich media M17G supplemented with $0.25 \mathrm{M}$ (left) and $0.50 \mathrm{M}$ (right) NaCl. Strain L. lactis LL00 harbors the empty vector pBV153, whereas the series LL03, LL04, LL05 and LL06 carry a trans copy of genes $c d a A^{l l}, g d p P^{l l}, c d a A^{e f}$ and $g d p P^{e f}$, respectively.

c-di-AMP homeostasis has been reported to be connected to cell wall metabolism, and in fact, CdaA is modulated by GlmM, an enzyme in charge of synthesizing the cell wall precursor glucosamine-1-phosphate (82). Moreover, gdpP mutants in L. lactis have also been found to be more resistant to sublethal concentrations of penicillin G (74). Taking this into account, response to sublethal concentrations of penicillin and ampicillin (two $\beta$-lactam antibiotics) was tested for strains LL03-06.

Once more, no significant effect was observed for strain LL04 and a growth disadvantage was evidenced for strain LL03 when media were supplemented with $0.25 \mu \mathrm{g} / \mathrm{ml}$ ampicillin or $0.10 \mu \mathrm{g} / \mathrm{ml}$ penicillin, showing a more severe effect for the latter case. On the other hand, even though both strains LL05 and LL06 manifested impaired growth in presence 
of $0.25 \mu \mathrm{g} / \mathrm{ml}$ ampicillin, strain LL06 grew more similar to the LL00 control strain when 0.10 $\mu \mathrm{g} / \mathrm{ml}$ penicillin was added to the medium instead Fig 19 .

So far, only the response to $\beta$-lactam antibiotics has been reported for $L$. lactis strains with mutations altering c-di-AMP intracellular levels. Since response to different stress factors could also allow the identification of other pathways related to c-di-AMP metabolism, growth curves in presence of compounds with a different mechanism of action was evaluated.

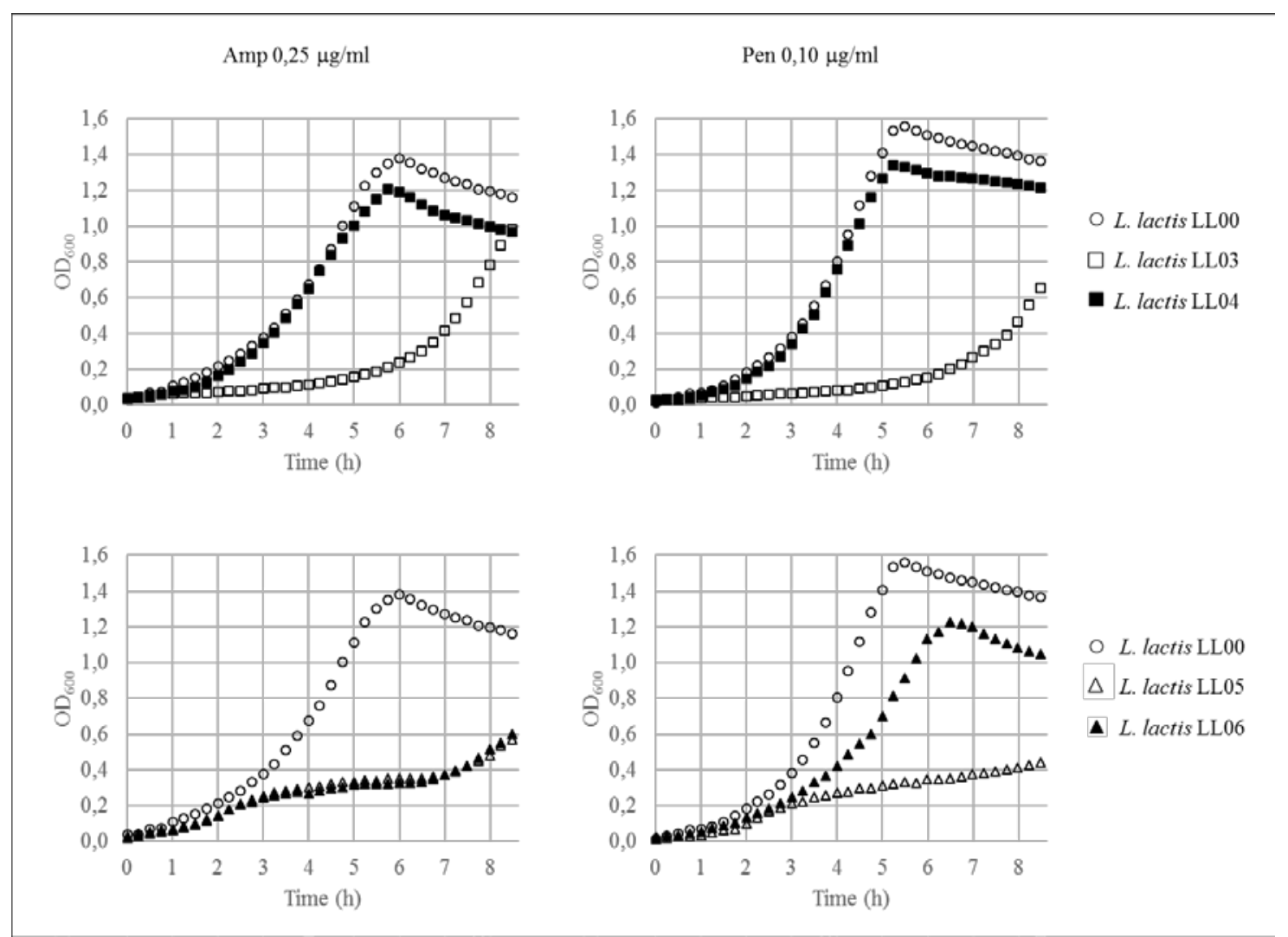

Fig 19 Response of strains LL00, LL03 - LL06 to $\beta$-lactamic antibiotics. Growth curves were performed at $30^{\circ} \mathrm{C}$ in rich media M17G supplemented with $0.25 \mu \mathrm{g} / \mathrm{ml}$ ampicillin (left) and $0.10 \mu \mathrm{g} / \mathrm{ml}$ penicillin (right). L. lactis LL00 harbors the empty vector pBV153, whereas the series LL03, LL04, LL05 and LL06 carry a trans copy of genes $c d a A^{l l}, g d p P^{l l}, c d a A^{e f}$ and $g d p P^{e f}$, respectively.

Vancomycin is a glycopeptide active against the second phase of cell wall synthesis, i.e. it binds to D-Ala-D-Ala ends of monomers being processed for its later addition to the cell wall. Nevertheless, once vancomycin is bound, the complex formed is no longer a substrate for subsequent enzymes and consequently lipid intermediates accumulate in the membrane (168). Considering that it affects a different stage of cell wall biosynthesis, its impact on cells with modified intracellular pool of c-di-AMP could give insights as regards the importance of this second messenger in the different stages. 
As Fig 20 shows, only strains LL03 and LL05, harboring an extra copy of $c d a A^{l l}$ and $g d p P^{l l}$, respectively, evidenced a negative impact on growth, when media were supplemented with $0.50 \mu \mathrm{g} / \mathrm{ml}$ vancomycin. E. faecalis enzymes seem not to affect the response, having a behavior similar to the control with the empty vector.

On the other hand, lysozyme is an enzyme capable of hydrolyzing the glycosidic bonds on the cell wall, releasing a disaccharide formed by acetylglucosamine and muramic acid (169). Its impact as an antimicrobial agent has long been studied, and it has also been reported that in L. monocytogenes the lack of CdaR increased resistance to this compound (170). Taking into account that in the same work it was proved that CdaR negatively regulates CdaA in this bacterium, this would mean that high amounts of c-di-AMP could favor lysozyme resistance. Therefore, this hypothesis was tested for the strains here developed.

In Fig 20, it can be observed that an extra copy of $g d p P$ gene (LL05 and LL06) increased susceptibility to lysozyme. At a concentration of $0.10 \mu \mathrm{g} / \mathrm{ml}$, LL05 and LL06 ( $g d p P^{l l}$ and $g d p P^{e f}$, respectively) strains are no longer able to grow in the time interval here presented, whereas the control strain reaches a final $\mathrm{OD}_{600}$ of 1.0. Taking into account that both strains are expected to produce higher amounts of GdpP proteins, and consequently lower their c-di-AMP levels inside the cell, these results agree with the ones previously described for $L$. monocytogenes.

Interestingly, different phenotypes were evidenced for strains harboring an extra copy of $c d a A^{l l}$ and $c d a A^{e f}$. Even though trans-expression of the former impedes growth of strain LL03, the phenotype observed due to expression of latter in strain LL04, agrees with the published results mentioned, since it confers a growth advantage with respect to the control LL00 strain, harboring the empty vector. 


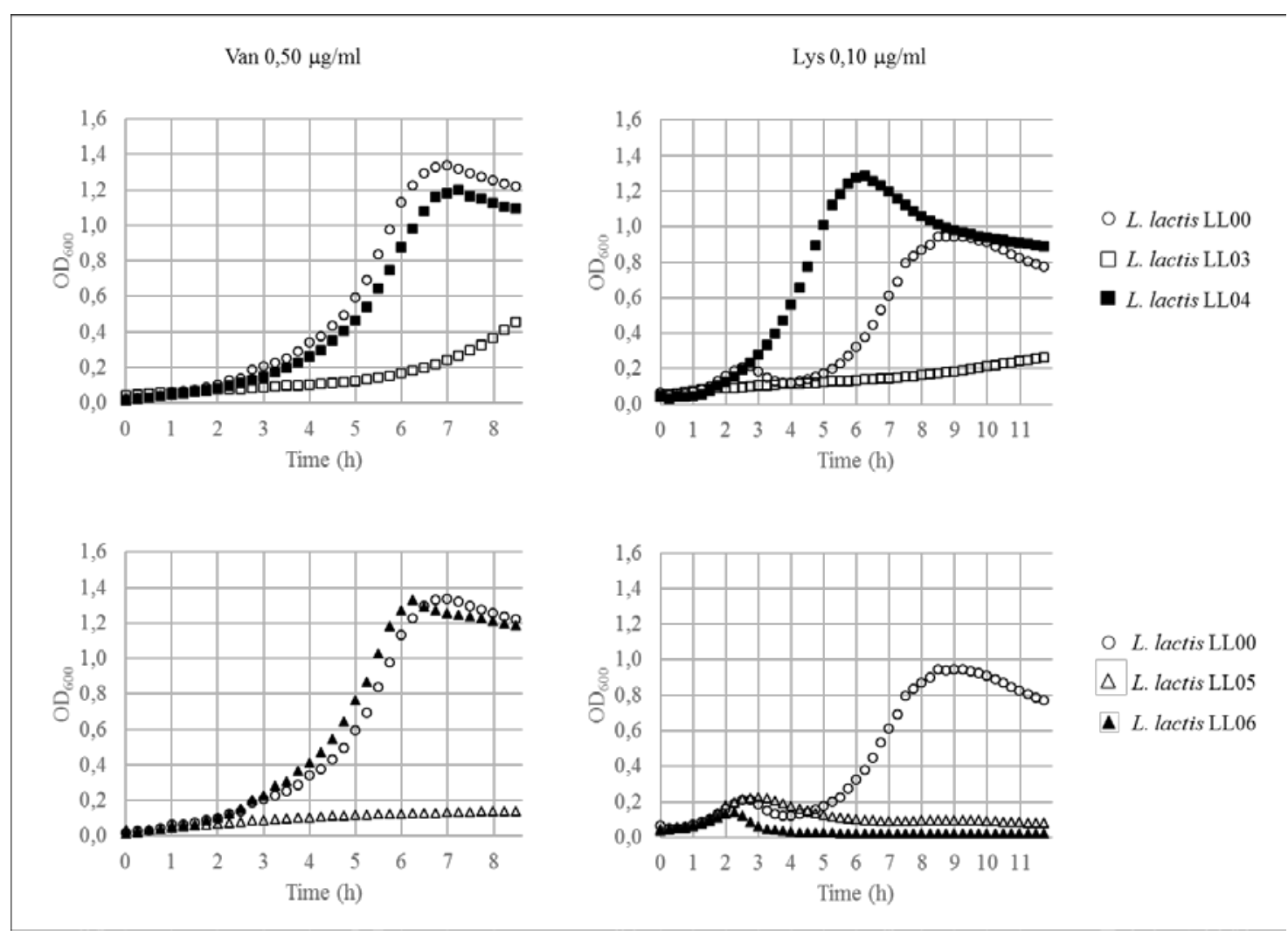

Fig 20 Impact of vancomycin and lysozyme on strains LL00, LL03 - LL06. Growth curves were performed at $30{ }^{\circ} \mathrm{C}$ in rich media M17G supplemented with $0.50 \mu \mathrm{g} / \mathrm{ml}$ vancomycin (left) and 0.10 $\mu \mathrm{g} / \mathrm{ml}$ lysozyme (right). L. lactis LL00 harbors the empty vector pBV153, whereas the series LL03, LL04, LL05 and LL06 carry a trans copy of genes $c d a A^{l l}$, $g d p P^{l l}$, $c d a A^{e f}$ and $g d p P^{e f}$, respectively.

Finally, the two isolated $g d p P$ mutants (strains LL07 and LL08) were also analyzed by phase contrast microscopy in order to evaluate differences in morphology. In Fig 21, images of bacteria during mid-exponential growth are presented (down), where a slight decrease in size can be seen for strain LL07, which showed smaller colonies. In addition, for both samples under study, well defined round shaped cells were evidenced, with no significant differences with respect to the control strain LL00 harboring the empty vector pBV153.

As previously mentioned, $g d p P$ mutants analyzed by Smith et al. showed improved growth at sublethal concentrations of the $\beta$-lactamic antibiotic penicillin. Therefore, both mutant strains LL07 and LL08 were grown in presence of ampicillin to further study possible phenotypic differences between them. In Fig 21, growth curves obtained in M17G supplemented with $5.0 \mu \mathrm{g} / \mathrm{ml}$ ampicillin are presented, where a detriment is evidenced for both strains. Interestingly, the opposite phenotype was observed in these conditions, and even a slight difference is observed in $\triangle g d p P$ strains LL07 and LL08, being the former more sensitive to the antibiotic than the latter. 


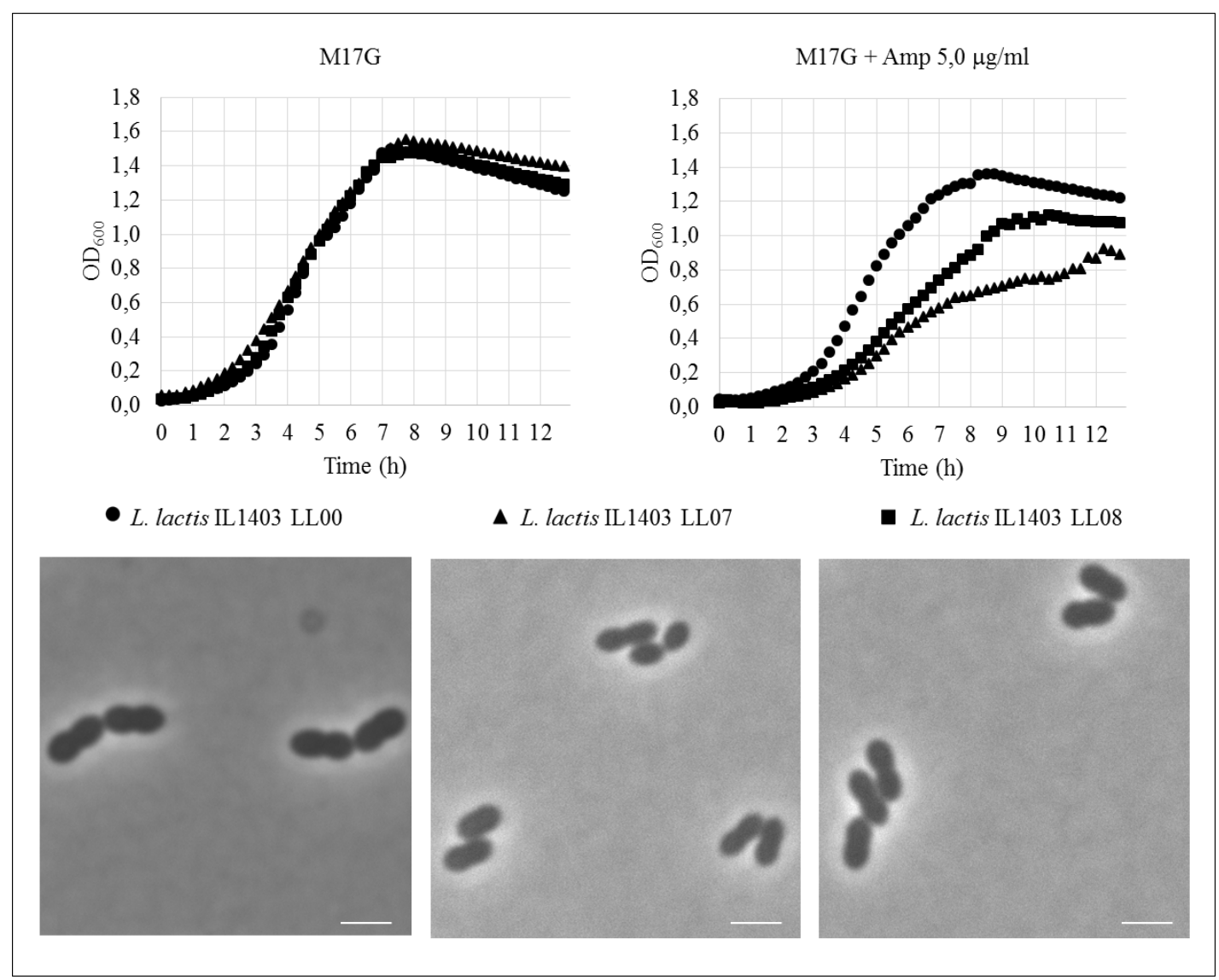

Fig 21 Phenotypic analysis of $\Delta \boldsymbol{g d p P}$ mutants Up: Growth curves performed in rich media M17G without (left) and with supplementation of $5.0 \mu \mathrm{g} / \mathrm{ml}$ ampicillin (right). Down: Phase contrast microscopy of strains LL00, LL07 and LL08. Bacteria were grown in M17G medium at $30{ }^{\circ} \mathrm{C}$ and samples were taken at mid-exponential phase. Scale bar, $2 \mu \mathrm{m}$.

\subsubsection{Intracellular levels of c-di-AMP are modified in strains $L$. lactis LL03-LL08}

After analyzing phenotypes and stability of strains developed in the previous section, their c-di-AMP intracellular levels were determined. As mentioned, pBV153 vector is induced at low $\mathrm{pH}$, which is why strains LL00, LL03-06 harboring this plasmid and its derivatives were grown at initial $\mathrm{pH}\left(\mathrm{pH}_{0}\right)$ of 7.0 and 5.5 for later c-di-AMP quantification. Briefly, cells were grown in M17G supplemented with $10 \mu \mathrm{g} / \mathrm{ml}$ Cm for strains LL00, LL03-06 and without antibiotic for strains LL07 and LL08. OD600 measurements were taken every hour to check growth (not shown). When $\mathrm{OD}_{600}$ reached 0.5, cells were harvested and pellets stored for c-diAMP extraction and determination. Results were normalized by the amount of protein present in the samples (see Materials and Methods).

Values obtained for strain LL00 as well as IL1403 indicate that basal levels of c-diAMP are in the range of 19.9 - 41.3 and 28.0 - 34.1 ng of c-di-AMP per mg of protein at $\mathrm{pH} 0$ 
7.0 and 5.5, respectively. These levels could be significantly increased when strain LL03 with plasmid pIQ101 harboring $c d a A^{l l}$ gene was grown at $\mathrm{pH}_{0}=7.0$. Average values obtained in these conditions, for three technical replicates were 334.0 and $350.0 \mathrm{ng}$ of c-di-AMP per mg of protein in biological duplicates (Fig 22). This means that at least 8 times higher values are possible to obtain in L. lactis IL1403 without reaching toxic levels. Furthermore, when plasmid was PIQ101 was induced at $\mathrm{pH}_{0}$ 5.5, c-di-AMP concentrations were almost doubled reaching average values of 698.4 and 652.5 ng of c-di-AMP per mg of protein in biological duplicates, more than 15 times higher than basal levels just mentioned.

Accordingly to results described in the previous section, where strain LL04 with plasmid pIQ102 harboring a copy of $c d a A^{e f}$ showed no particular phenotypes when grown in presence of different stress factors, no impact on c-di-AMP levels was manifested in this experiment either. At both $\mathrm{pH}_{0}$ similar values to the ones observed for the control strain LL00 were obtained, suggesting once more that $\mathrm{CdaA}^{\text {ef }}$ enzyme is not active under the working conditions of this study.

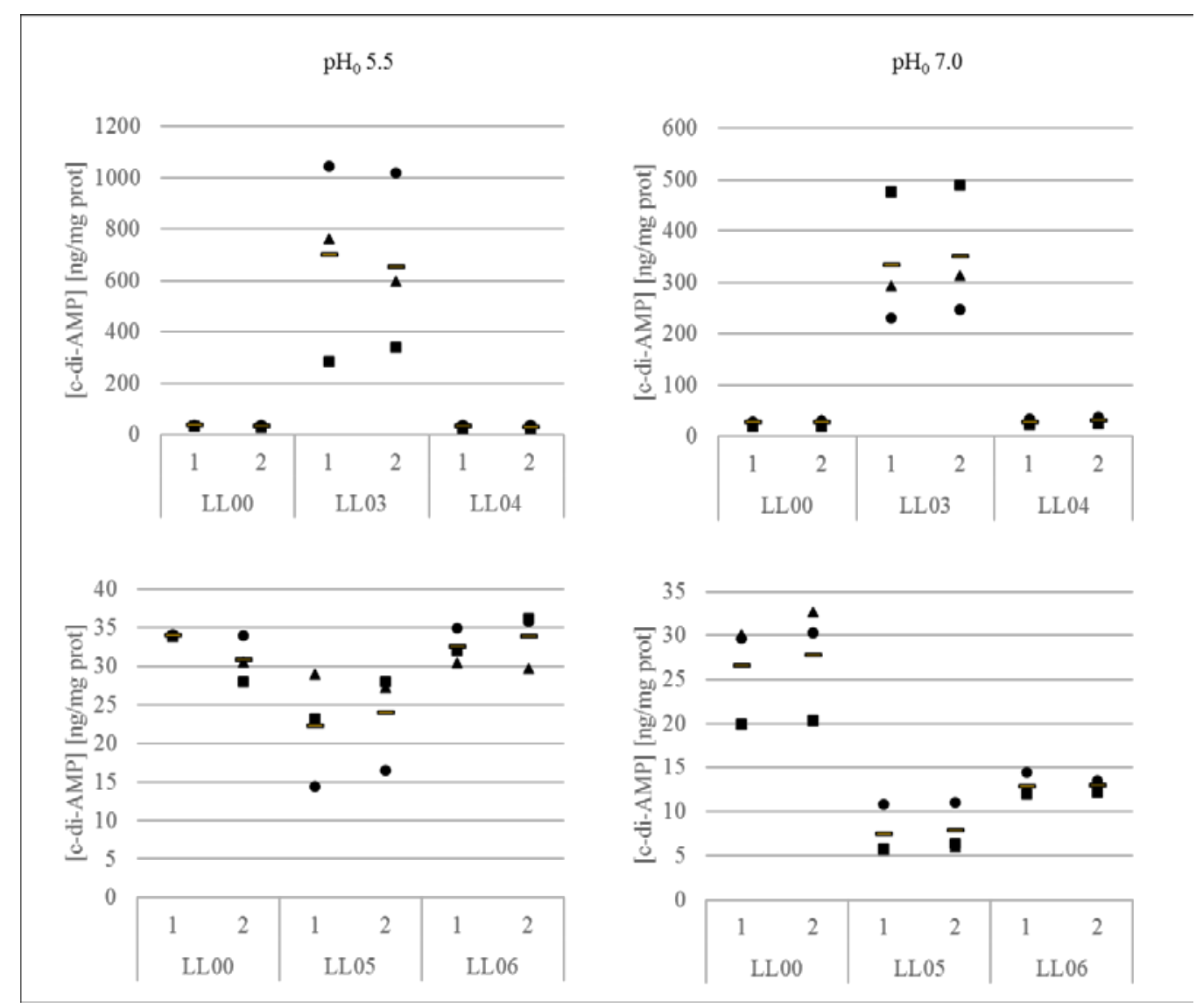

Fig 22. c-di-AMP intracellular levels of strains LL00, LL03-06. Cells were grown in rich M17G medium and harvested at OD $=0.5$ for c-di-AMP extraction and quantification. Strains were grown at $\mathrm{pH}_{0}=5.5$ (left) and 7.0 (right). Circles, squares and triangles correspond to technical triplicates. 1 and 2 indicate biological duplicates. 
On the other hand, the presence of a trans copy of $g p d P^{l l}$ gene in strain LL05 allowed reduction of intracellular c-di-AMP values to 14.4 - 29.0 and 5.7 - $11.0 \mathrm{ng}$ of c-di-AMP per mg of protein at $\mathrm{pH}_{0}$ of 5.5 and 7.0, respectively. These values indicate that the enzyme could be more active at $\mathrm{pH}_{0} 7.0$, and coincide with the ones obtained for strain LL06, which were also lower at $\mathrm{pH}_{0} 7.0$, although results above described suggest a higher induction of the expression system at $\mathrm{pH}_{0} 5.5$.

Interestingly, different results were obtained for the two $g d p P$ mutant strains LL07 and LL08. In the first case, values in the range of wild type strain IL1403 were registered (Fig 23), whereas in the second case, intracellular c-di-AMP concentrations were almost doubled (63.6 $-85.1 \mathrm{ng}$ of c-di-AMP per mg of protein). It is important to mention that the fact that c-di-AMP levels are held close to wild type ones even in the absence of $g d p P$, suggests that there could be another c-di-AMP degrading enzyme encoded in L. lactis genome.

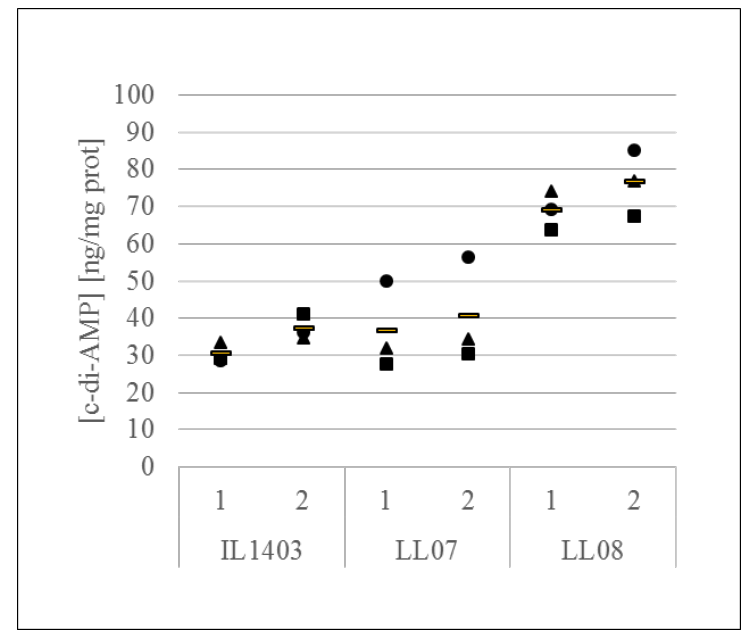

Fig. 24 Intracellular concentrations of c-di-AMP in $L$. Iactis $\mathbf{A g d p P}$ mutants. Strains were grown at $\mathrm{pH}_{0}=7.0$ and haversted when $\mathrm{OD}_{600}$ reached 0.5. Different c-di-AMP values were obtained for both $g d p P$ mutant strains. Circles, squares and triangles correspond to technical triplicates. 1 and 2 indicate biological duplicates

Finally, the impact of ampicillin on c-di-AMP levels was analyzed for strains LL05 and LL06. Both were grown in rich media M17G in presence of $0.25 \mu \mathrm{g} / \mathrm{ml}$ and since LL06 in the previous section evidenced more resistance to $\beta$-lactamic antibiotics, a concentration of 0.5 $\mu \mathrm{g} / \mathrm{ml}$ was selected in this case as well. Fig. 25 shows that ampicillin supplementation of media causes a decrease of c-di-AMP levels, being the lowest concentration in the range 3.7 - $12.4 \mathrm{ng}$ of c-di-AMP per mg of protein at $\mathrm{pH}_{0} 7.0$ for strain LL06, harboring a trans copy of $c d a A^{e f}$ gene. 


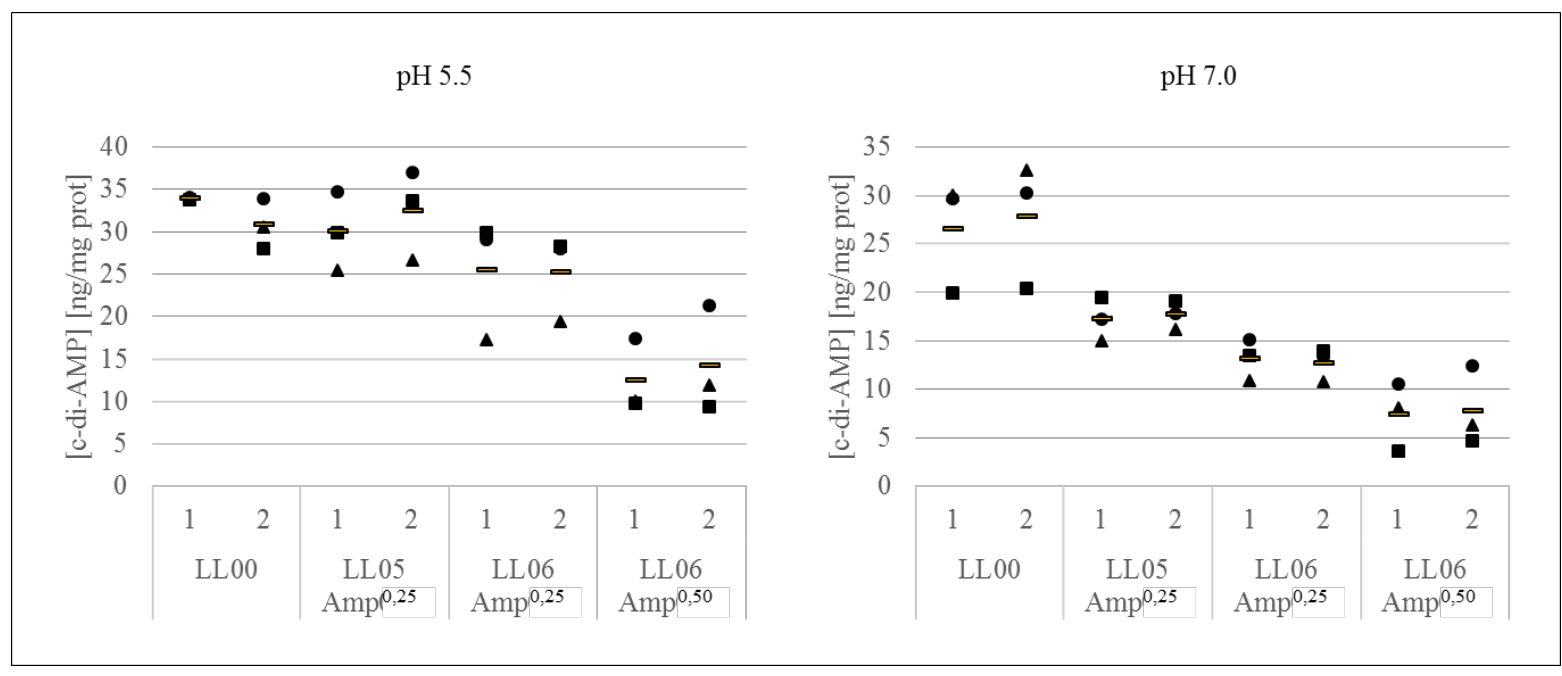

Fig. 25 Ampicillin impact on strains L. lactis LL05 and LL06. Strains were grown in in rich medium M17G at $\mathrm{pH}_{0}=5.5$ (left) and $\mathrm{pH}_{0}=7.0$ (right). c-di-AMP levels were taken at $\mathrm{OD}_{600}=0.5$. The lowest c-di-AMP levels are seen for strain LL06 at $\mathrm{pH}_{0}=7.0$. Circles, squares and triangles correspond to technical triplicates. 1 and 2 indicate biological duplicates.

Altogether, these results allowed us to establish working conditions where c-di-AMP are increased more than 15 times. Therefore, for the immunological experiments in the following chapter, strain LL03 as well as the system of expression with pIQ101 vector induced at $\mathrm{pH}_{0}=5.50$ were selected.

\subsubsection{Phosphodiesterases c-di-AMP in L. lactis}

In the previous section, the maintenance of wild type c-di-AMP intracellular levels in $\triangle g d p P$ mutant strains suggests the possibility of other enzymes encoded in L. lactis genomes capable of degrading this second messenger. As mentioned in the introduction, the second family of c-di-AMP phosphodiesterases, which comprises B. subtilis $\mathrm{PgpH}$, contains an HDdomain and a His-Asp motif. Even though homologues are found in many members of firmicutes, a protein blast in L. lactis using this protein as query, threw no positive results.

Another group of smaller phosphodiesterases presents only a soluble DHH/DHHA1 domain and was found for example in S. pneumoniae and B. subtilis, although in the latter this enzyme does not degrade c-di-AMP. This time the protein threw a protein encoded by yheB of L. lactis IL1403 with 48\% identity to B. subtilis NrnA and 64\% to S. pneumoniae Pde2, albeit no further information about its function is available.

With the aim of starting a characterization of YheB protein and evaluating whether it can degrade c-di-AMP, yheB gene from L. lactis IL1403 and NZ9000 was cloned in the 
expression vector pWH844 (see Table XVI Plasmids). This vector allows E. coli overexpression of heterologous genes with an N-terminal His6x-tag for purification purposes. Moreover, GdpP proteins from both strains were cloned as well to be used as positive control of c-di-AMP hydrolyzing enzymes, although these experiments could not be performed due to time restraints. Resulting plasmids pIQ401, pIQ402, pIQ403 and pIQ404 containing genes $y h e B^{N Z}, g d p P^{N Z}$, $y h e B^{I L}$ and $g d p P^{I L}$ were electroporated in E. coli BL21, originating strains E. coli YB01, GP01, YB02 and GP02, respectively.

After checking all gene sequences, an expression test was performed in rich LB medium at $37^{\circ}$. Briefly, strains were grown until OD 600 reached 0.5 , when $0.5 \mathrm{mM}$ IPTG was added and cultures were incubated for three more hours. Fig 26 shows an SDS-PAGE were bands of circa $35 \mathrm{kDa}$ and $74 \mathrm{kDa}$ evidence the presence of YheB and GdpP for strains YB01 - 02 and GP 01-02, respectively.

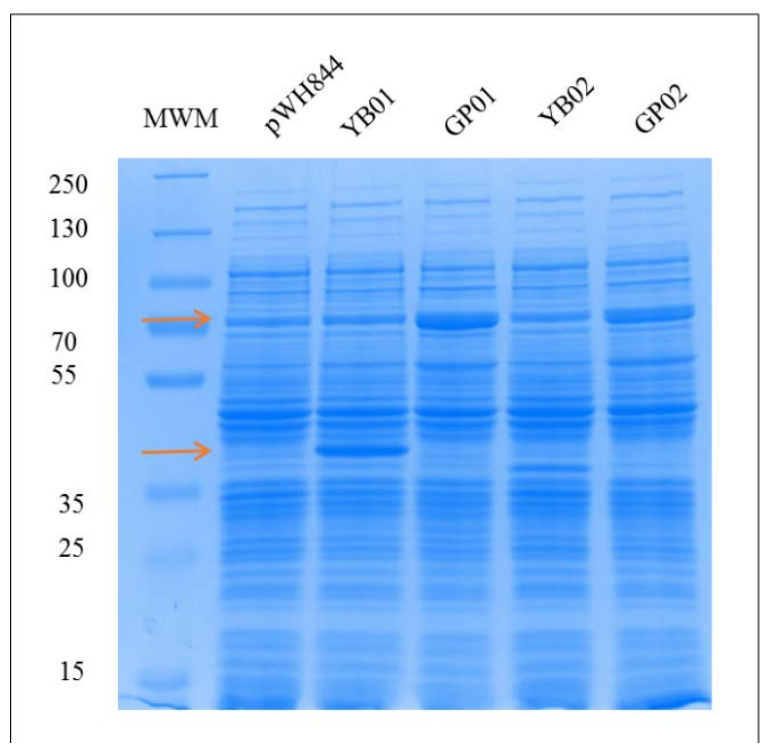

Fig 26 Expression test of strains $E$. coli YB01 -02 and GP01 -02. SDS-PAGE gel after Coomasie staining. Arrows indicate the presence of expression bands corresponding to proteins $\mathrm{YheB}^{\mathrm{NZ}}$ and $\mathrm{YheB}^{\mathrm{IL}}$ in lanes 2 and 4, respectively as well as $\mathrm{GdpP}^{\mathrm{NZ}}$ and $\mathrm{GdpP}^{\mathrm{IL}}$ in lanes 3 and 5, respectively. MWM: molecular weight marker.

Once production of proteins was confirmed, both YheB proteins were purified using a $\mathrm{Ni}^{2+}$-NTA resin (see Materials and Methods). Fig 27 shows SDS-PAGE gels were the purification process is evidenced and the proteins of interest are present in fractions E3 and E4 for $\mathrm{YheB}^{\mathrm{IL}}$ (left) and E2, E3 and E4 for $\mathrm{YheB}^{\mathrm{NZ}}$ (right). Afterwards, proteins were further purified via size exclusion chromatography (SEC) by use of the Äkta system to eliminate imidazole of the media. Samples were then concentrated and frozen in liquid nitrogen to be finally stored at $-80^{\circ} \mathrm{C}$ until used. 


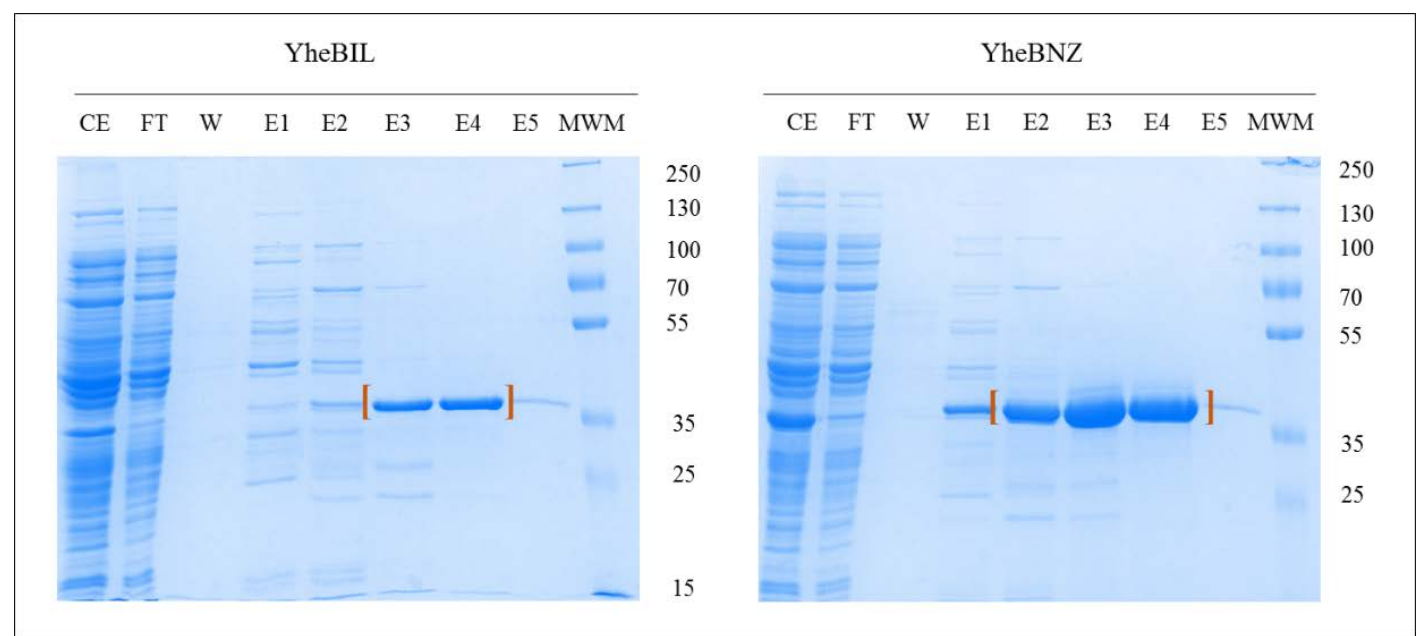

Fig 27 Purification of $Y_{h e B}{ }^{\mathrm{IL}}$ and $\mathrm{YheB}^{\mathrm{NZ}}$ proteins. Elutions further purified via size exclusion chromatography are highlighted in orange. CE: crude extract, FT: Flow through, E: elution, MWM: molecular weight marker. E1 - E5: 30 - 50 - 100 - 200 - 500 mM imidazole, respectively.

A first approach into function determination of YheB proteins was performed via the BNPP assay. bis(4-nitrophenyl)phosphate can be cleaved by phosphodiesterases yielding paranitrophenol, which production can be followed colorimetrically at $410 \mathrm{~nm}$ ((171), Materials and Methods). Reactions were performed at different $\mathrm{pH}$ and adding $\mathrm{Mn}^{2+}$ to the media to analyze optimal conditions for further studies. As seen in Fig 28, YheB ${ }^{\mathrm{NZ}}$ enzyme seems to be more active, and both proteins evidenced increased activity when $\mathrm{pH}$ was raised. These results suggest that YheB protein could be a c-di-AMP phosphodiesterase.

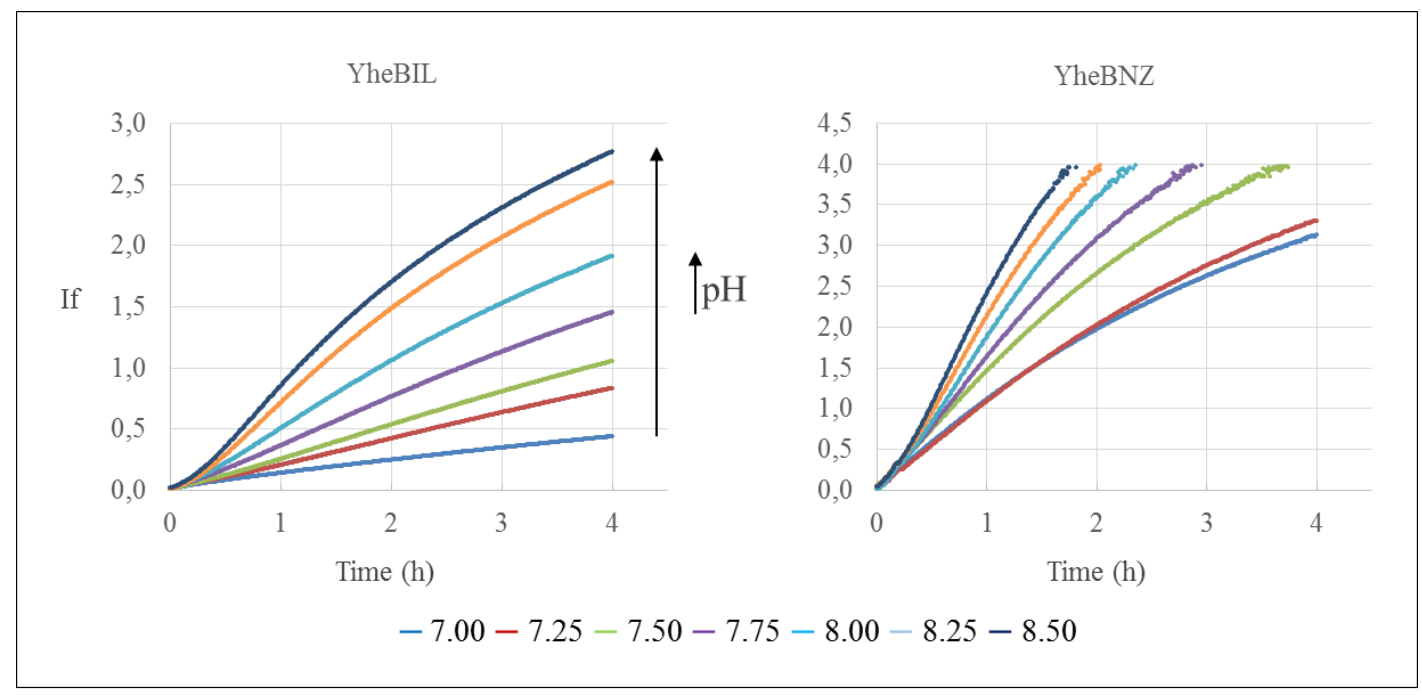

Fig 28 BNPP assay for YheB proteins. YheB ${ }^{\mathrm{IL}}$ (left) and $\mathrm{YheB}^{\mathrm{NZ}}$ (right) were analyzed via BNPP assay. Reads were performed for four hours in a microplate reader. The arrow shows increasing $\mathrm{pH}$ in the reaction media. Both graphs show higher activity when $\mathrm{pH}$ is raised. 


\subsubsection{Conclusions}

The aim of this chapter was to evaluate the physiological consequences when c-di-AMP intracellular pools are altered in L. lactis IL1403. Depletion of this secondary messenger via $c d a A$ deletion was not possible in rich, nor in minimal media. The strategy was thus changed and responses obtained along the different experiments here presented, evidenced overexpression of heterologous and homologous $c d a A$ and $g d p P$ genes by use of pBV153 vector.

In general, strains here developed were more sensitive on rich media and upon the presence of stress factors such as $\mathrm{NaCl}$, antibiotics and high temperature. The only exception was $c d a A^{e f}$ overexpressed in presence of $0.1 \mu \mathrm{g} / \mathrm{ml}$ lysozyme, which provided a growth advantage. Since the resulting $\mathrm{CdaA}^{\mathrm{EF}}$ enzyme showed no great impact on c-di-AMP levels, it could be said that in this conditions, its activity is diminished. If this is the case, detrimental phenotypes obtained in the other growth curves could be due to a system were c-di-AMP levels are too low or too high, which has already been reported to result in impaired growth (172).

The highest average values of c-di-AMP obtained in this work reached 698.4 and 652.5 ng of c-di-AMP per mg of protein in biological duplicates for strain LL03. Although these differences could be due to methodological variations, an increment of more than 15 times with respect to basal levels was obtained. L. lactis LL03 was therefore selected for further studies on the development of a live vaccine prototype in next chapter.

On the other hand, reduction of c-di-AMP intracellular levels by expression of $g d p P$ genes was more significant at $\mathrm{pH}_{0}$ 7.0. Despite the fact that the induction of the expression system used is higher when $\mathrm{pH}$ is lowered, these results lead to the hypothesis that GdpP enzyme activity in L. lactis would prevail when extracellular $\mathrm{pH}$ is closer to physiological values. Moreover, the biggest impact in this way was accomplished by strain LL06 grown in rich media M17G supplemented with $0.50 \mu \mathrm{g} / \mathrm{ml}$ ampicillin. Average values of technical triplicates were 7.4 and $7.8 \mathrm{ng}$ of c-di-AMP per mg of protein, linking the impact of a $\beta$-lactamic antibiotic to c-di-AMP levels in L. lactis IL1403.

Finally, the fact that $\Delta g d p P$ mutants are able to maintain c-di-AMP levels close to wild type ones, supports the hypothesis of the existence of at least another enzyme capable of hydrolyzing c-di-AMP. The main candidate found in this work is the uncharacterized YheB protein, which was proved here to degrade BNPP. Nevertheless, this compound is an unspecific 
substrate for phosphodiesterases, and even though phosphodiesterase activity is evidenced here, and proved to increase at higher $\mathrm{pH}$, further specific studies are needed to confirm it can degrade c-di-AMP (68). 


\subsection{Development of an oral subunit vaccine prototype against Chagas disease}

\subsubsection{Antigen design and production in L. lactis}

As mentioned in the introduction, T. cruzi trans-sialidase (TS) enzyme plays a vital role upon infection of the mammal host, since it catalyzes the transfer of sialic acid from the host glycoconjugates to the terminal $\beta$-galactopyranosyl residues of mucin-like molecules on the parasite's cell surface. It is also involved in different pathways leading to successful parasite infection and down-regulation of the host immune response (173). Therefore, TS was selected to develop a suitable antigen for the vaccine prototype against Chagas disease.

The high performance of the whole TS protein as a T. cruzi antigen in vaccine prototypes has already been reported $(174,175)$. Nevertheless, considering that protein overexpression in LAB is not as straight forward as normally is in other bacterial models such as E. coli, an abbreviated fraction of the antigen was selected instead of the entire TS protein. In addition, the engineered $L$. lactis strain was designed to co-express the synthesizing enzyme of the adjuvant as well, which adds a detrimental factor to bacterial growth and protein expression. Altogether, in order to facilitate protein co-expression in L. lactis, the smallest TS protein size possible was selected, having at the same time the highest epitope concentration able to trigger a TS-specific immune response.

Based on these criteria, an epitope prediction within the complete TS antigen sequence was performed by Ivan Marcipar group in Laboratorio de Tecnología Inmunológica, Facultad de Bioquímica y Ciencias Biológicas, UNL (Universidad Nacional del Litoral). T epitopes against H-2Kd MHC-I were selected for prediction, and a total of 7 epitopes were identified, with four of them in the central region of the protein, ranging from amino acid 326 to 497. Interestingly, IYNVGQVSI epitope was included in this region, which has been described as the main MHC-I T-cell epitope that provides protection against T. cruzi infections in BALB/C mice $(176,177)$. On the other hand, B-epitopes have shown to comprise key amino acids responsible for enzymatic catalysis (178), which is why antibodies developed against this region may potentially neutralize TS activity. Therefore, prediction of B epitopes was also carried out, identifying 15 out of the 50 epitopes with the highest score within the same central 
region. These predictions lead to the final TS fragment selection, ranging from amino acid 326 to 497 (Fig. 29).

Given that synthesis of heterologous proteins may result in amino acid unbalance, the designed peptide was submitted to GenScript synthesis service (U.S.A.) to optimize codon usage for L. lactis and to incorporate $\mathrm{NcOI}$ and HindIII restriction sites required for cloning, as well as an N-terminal His6x tag to allow detection by Western blot. The resulting gene was named tscf and cloned into the nisin inducible pNZ8048 vector previously described (pNZTS, Table XVI, Fig. 29), to be finally electroporated in L. lactis NZ9000 (strain NZTS, Table XV). After checking the genetic construction by sequencing, several conditions of expression were tested. Protein overproduction was proved in some of them, but it was not possible to detect it again in new trials of expression (not shown).

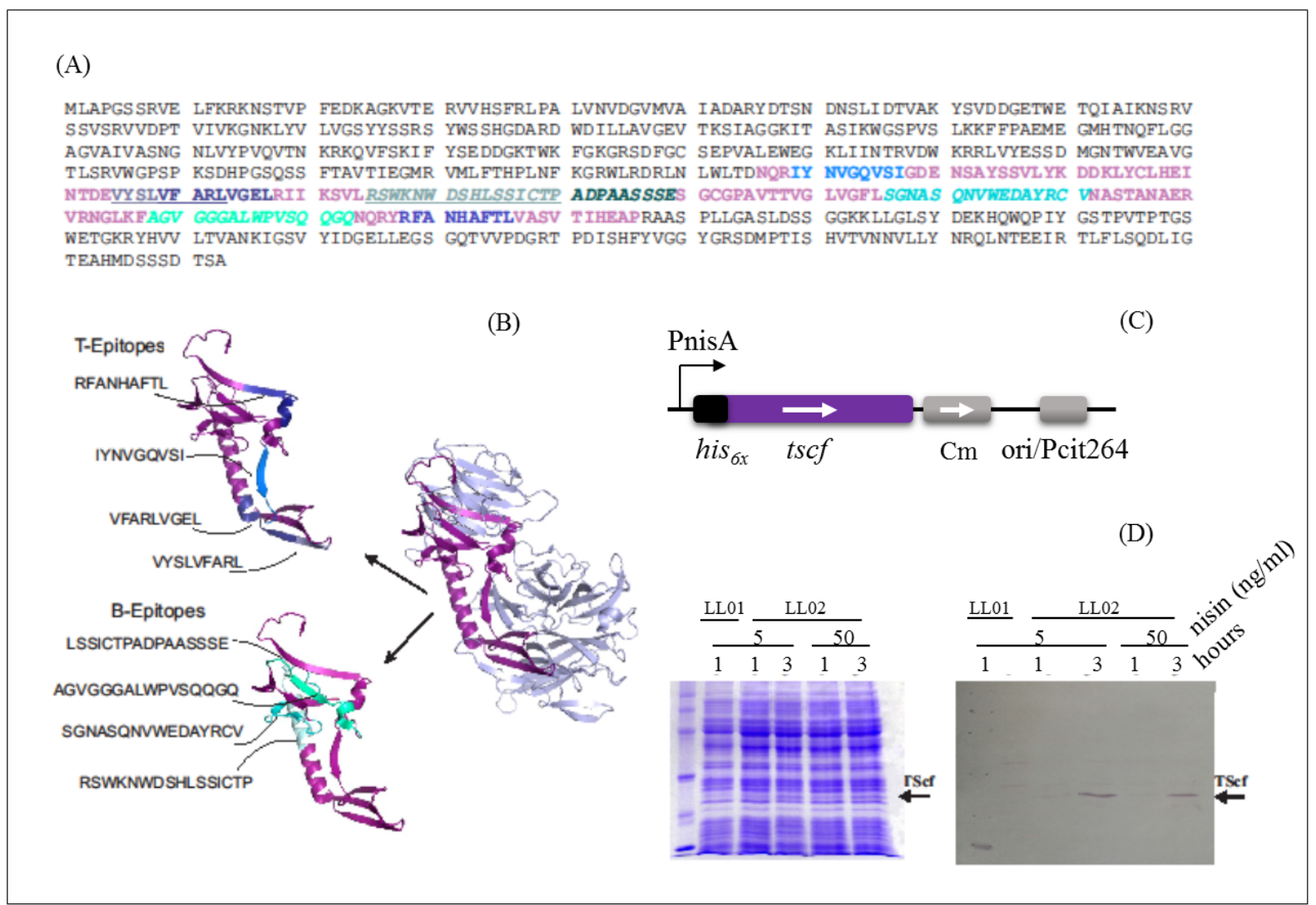

Fig. 29 Development of an optimized TS derived antigen. A: complete sequence of trans-sialidase protein. The selected fragment is highlighted in purple and colored amino acids within this sequence indicate epitopes. Underlined amino acids refer to superimposed epitopes. B: Structure modelling of whole TS (right) and the two sections conforming the synthetic antigen (left). C: cloning representation of his ${ }_{6 x}-t s c f$ in plasmid pNZ8048. D: Expression check by SDS-PAGE (left) and Western blot analysis (right).

As mentioned in the introduction, L. lactis clpP-htrA strain favors expression of heterologous genes since it lacks the two major proteases ClpP and HtrA, reducing degradation 
of heterologous proteins. Plasmid pNZTS was electroporated in this strain, resulting in strain LL02 (Table XV), and this time stable overproduction of TScf peptide was obtained. Briefly, cells were grown in 3 liters of M17G broth at $30^{\circ} \mathrm{C}$ until OD 600 reached 0.50 and induced with 5 or $50 \mathrm{ng} / \mathrm{ml}$ nisin during 1 or 3 hours. As shown in Fig. 29, Western blot analysis using antihis antibodies allowed visualization of a clear band when cultures were induced for 3 hours. Protein identity was determined by mass spectrometry (MS/MS), confirming overproduction of TScf.

\subsubsection{Immune response after mucosal co-administration of $L$. lactis strains producing TScf antigen and c-di-AMP adjuvant}

Once a strain able to produce the designed antigen (LL02) as well as a strain synthesizing high adjuvant levels (Chapter II, strain LL03) were obtained, the next step was to evaluate the effectiveness of their co-administration, as proof of concept for the development of a new prototype of mucosal vaccine. In collaboration with Dra. Ana Rosa Perez's group, from the Instituto de Investigación Clínica y Experimental de Rosario (IDICER CONICETUNR), three successive oral immunizations were performed in mice (see Materials and Methods). Studied groups were: Non-immunized (NI, mice receiving only PBS); LL02, mice immunized with bacteria producing the TScf antigen (L. lactis LL02, Table XV); LL02+LL03 group, mice co-administered with $L$. lactis LL02 plus bacteria over-producing c-di-AMP ( $L$. lactis LL03, Table XV). L. lactis LL01 strain harboring the empty pNZ8048 plasmid was also orally administered as control. In addition, since earlier studies showed a higher specific antiTS cellular response after immunization with TS plus ISCOMATRIX adjuvant by subcutaneous immunization (174), for comparative purposes a group of mice was also immunized with purified TScf adjuvanted with ISPA (C+, Gold standard group), an ISCOMATRIX type adjuvant developed by Dr. Marcipar group (152).

As shown in Fig. 30, 15 days after the last immunization, 48 hours footpad testing showed that L. lactis LL02+LL03 immunized group elicited a clear TS-specific cell-mediated immune response compared to PBS and LL01, while this difference is less significant compared with the L. lactis LL02 group. Interestingly, the increase in footpad thickness were similar between L. lactis LL02+LL03 and the Gold standard group. These results support that TScf sequence contains MHC-I T-cell epitopes, as was previously mentioned, but also suggest that 
orally-administered L. lactis over-expressing cdaA gene (LL03) could be used as immune stimulator of the response against this antigen.

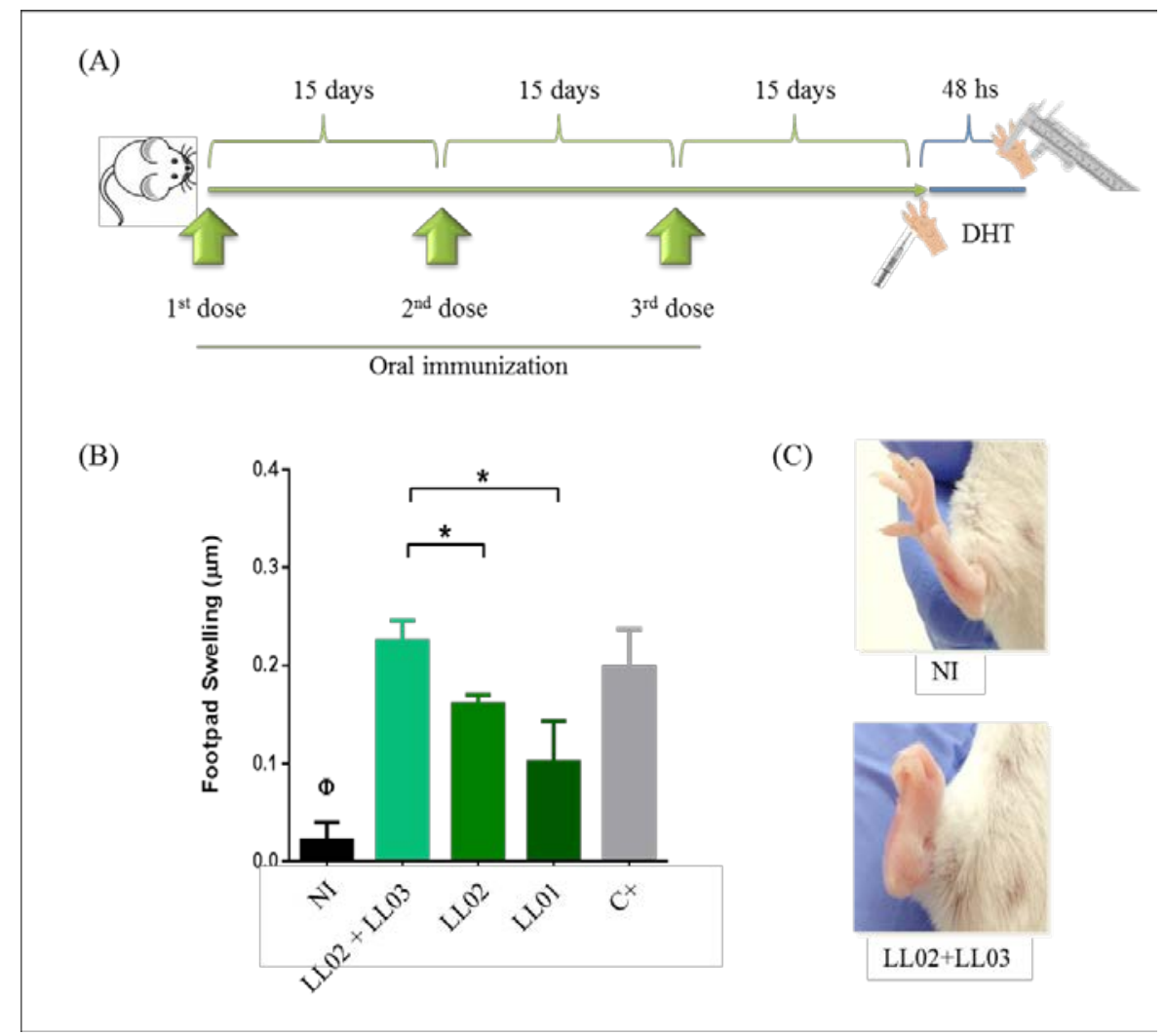

Fig. 30 Graphic representation of BALB/C mice oral immunization protocol. A: Oral immunization scheme. Three doses were administered with 15 days intervals. 48 hours after the last immunization, footpad swelling and DHT were measured. B: Co-administration of LL02+LL03 shows a significant difference with respect to LL02 as well as LL01 groups. C: Morphological difference in footpad swelling in non-immunized mice (up) and LL01+LL02 group (down). NI: non-immunized.

\subsubsection{A single engineered L. lactis strain producing c-di-AMP antigen and TScf adjuvant: a vaccine prototype.}

Aiming to the development of a fully integrated mucosal vaccine prototype, a single vector carrying both genes encoding the TScf antigen and the CdaA enzyme responsible for cdi-AMP production was designed. The first attempt to reach this consisted on amplifying from pIQ101 plasmid a DNA region containing the Pcit promoter, the $c d a A$ gene and its terminator. After several unsuccessful attempts of cloning this fragment in the BglII restriction site upstream the Pnis promoter in vector pNZTS, the strategy was changed. Instead, TScf coding gene was amplified including the Pnis promoter and terminator of pNZTS plasmid and subcloned in the SalI-PstI restriction sites of vector pIQ101. The resulting p10TS plasmid 
containing both genes was finally electroporated in L. lactis clpP-htrA strain, and the resulting strain was named L. lactis LL10 (Fig 31 and Table XV).

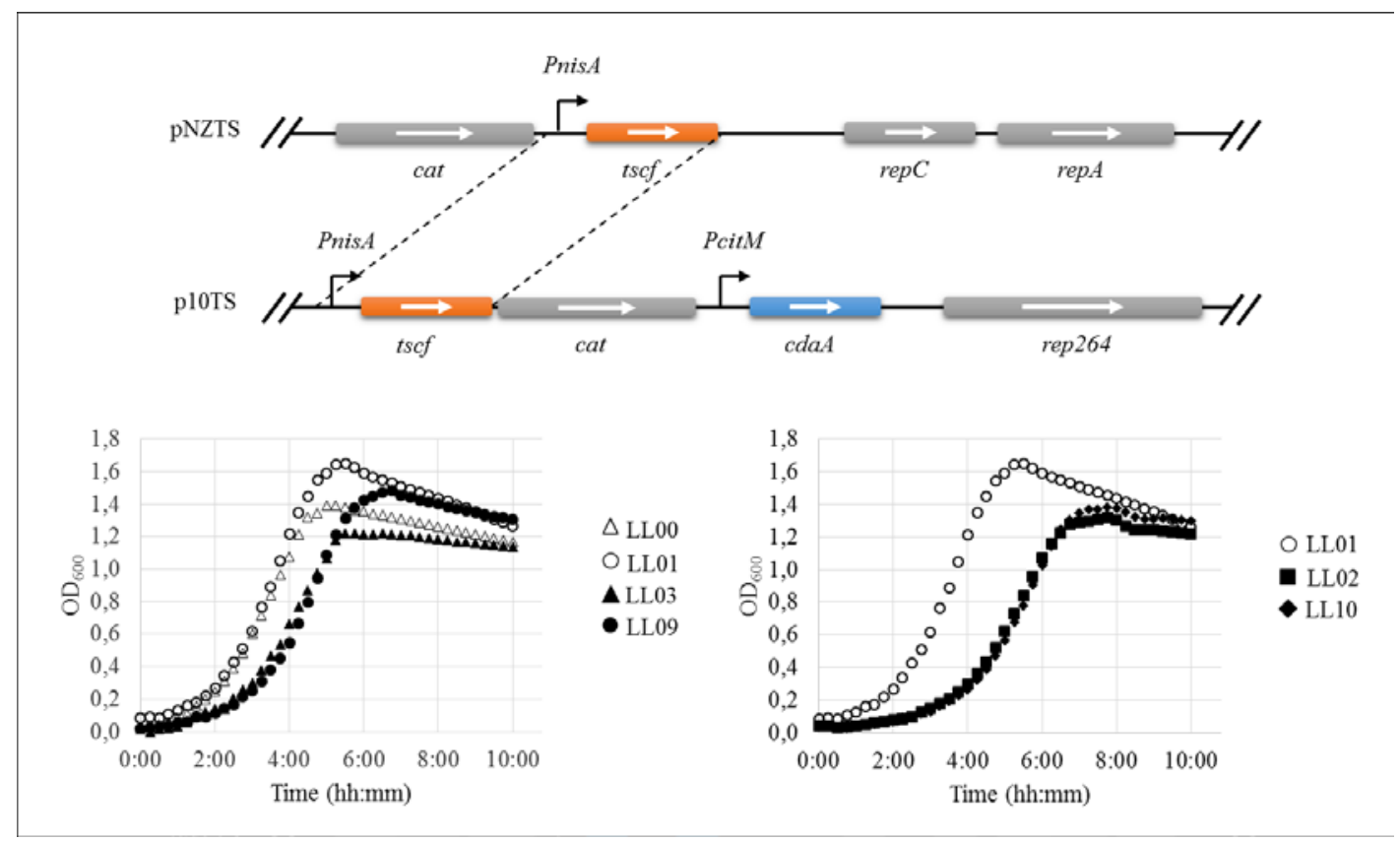

Fig 31 L. lactis LL10 strain construction and growth response. Up: cloning scheme for p10TS obtaining. This plasmid contains tscf gene under PnisA control and cdaA gene under PcitM control. Down: Growth curves performed in rich medium M17G. LL00: L. lactis IL1403 + pBV153, LL01: L. lactis NZ9000 clpP-htrA + pNZ8048, LL02: L. lactis NZ9000 clpP-htrA + pNZTS, LL03: L. lactis IL1403 + pIQ101, LL09: L. lactis NZ9000 clpP-htrA + pIQ101, LL10: L. lactis NZ9000 clpP$h$ trA + p10TS

In order to standardize conditions for the following immunological experiments, plasmid pIQ101 harboring cdaA gene was electroporated in strain L. lactis clpP-htrA, originating strain LL09. Since the main target of this chapter is the formulation of a live vaccine, comparative growth curves were performed to check response of L. lactis IL1403 and L. lactis clpP-htrA strains harboring a trans copy of $c d a A$ (Fig 31, down - left). On the other hand the same comparison was done for L. lactis clpP-htrA derivative strains harboring tscf gene (LL09) and both tscf and cdaA genes (LL10). Fig 31 shows similar responses in all cases, with homologous behavior for L. lactis IL1403 and L. lactis clpP-htrA derivative strains.

Moreover, and due to the presence of two different induction systems in strain LL10, an expression test was performed. In the previous chapter, the highest intracellular concentration of c-di-AMP was obtained at $\mathrm{pH}_{0}=5.5$, and cultures were grown until $\mathrm{OD}_{600}$ reached 0.50 . Since expression of $t s c f$ was induced once OD $600=0.50$ and cultures were then incubated for 3 more hours, the following step was to set up effective conditions for both systems. Therefore, nisin induction for strain LL10 was tested at two time points: simultaneously with inoculation 
$(\mathrm{OD}=0.05)$ and at $\mathrm{OD}_{600}=0.30$, until a final $\mathrm{OD}_{600}$ of 0.50 for both cases. Three nisin concentrations were also evaluated; 5, 15 and $50 \mathrm{ng} / \mathrm{ml}$, and strain LL02 induced with $0.5 \mathrm{ng} / \mathrm{ml}$ was used as control, for this was the condition in which TScf production was obtained in section 3.3.1.

As seen in Fig 32, the highest overproduction of TScf was evidenced in the crude extract as well as the supernatant fractions when $15 \mathrm{ng} / \mathrm{ml}$ nisin was added to growth media at the moment of inoculation $\left(\mathrm{OD}_{600}=0.05\right)$.

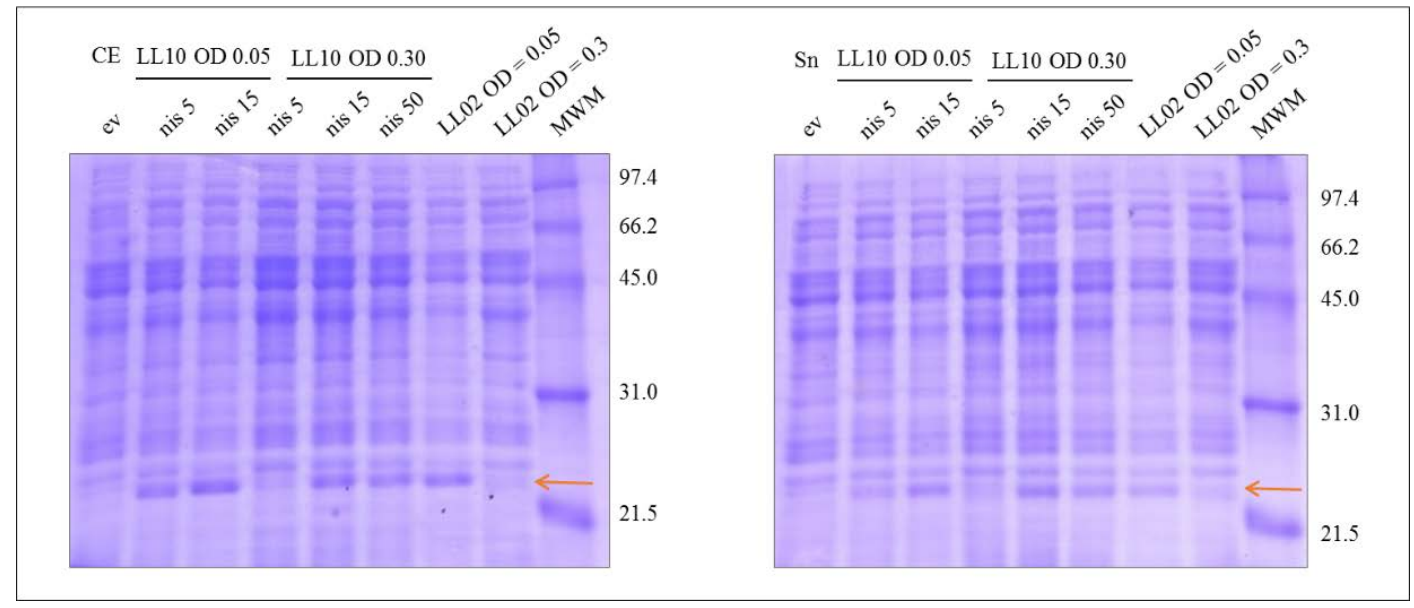

Fig 32 Synthesis of TScf in L. lactis LL10. Cultures of L. lactis LL10 were grown in rich M17G media supplemented with different nisin concentrations and at different times. Cells were harvested at $\mathrm{OD}_{600}=0.5$ in all cases. Crude extract (CE, left) and supernatant (Sn, right) fractions evidence a greater expression when $15 \mathrm{ng} / \mathrm{ml}$ nisin were added simultaneously with media inoculation. Orange arrows indicate TScf bands. ev: empty vector pNZ8048, MWM: molecular weight marker.

In conclusion, for the immunological experiments performed in the following section inocula were prepared as follows: 2 liters of fresh M17G medium supplemented with the corresponding antibiotics and $15 \mathrm{ng} / \mathrm{ml}$ nisin were inoculated with the desired strains at $\mathrm{OD}_{600}=0.05$ and incubated at $30{ }^{\circ} \mathrm{C}$ until $\mathrm{OD}_{600}$ reached 0.5. Cultures were then harvested and washed with PBS, to be finally resuspended at final OD600 of 2-3.109 CFU/100 $\mu \mathrm{l}$. An expression check to corroborate TScf presence was finally performed and aliquots were stored at $-80{ }^{\circ} \mathrm{C}$ until used (see Materials and Methods). 


\subsubsection{Immune response of engineered $L$. lactis co-expressing antigen and adjuvant after mucosal administration}

With a similar protocol to the one used in section 3.3.2., BALB/C mice were inoculated to analyze the in vivo cell-mediated immune response of strain LL10 developed in the previous section. This time inoculated groups were: Non-immunized (NI, mice receiving only PBS); LL10, mice immunized with L. lactis LL10 (co-producing TScf and CdaA), LL02, a group of mice receiving bacteria producing the TScf antigen alone (L. lactis LL02) and LL09, mice immunized only with the c-di-AMP producer strain (L. lactis LL09). A group of mice immunized with L. lactis LL01 strain harboring the empty pNZ8048 plasmid and a group receiving TS plus the ISCOMATRIX adjuvant were included as control.

A scheme of three doses separated by 15 days intervals, similar to the one described in the previous section was again used (Fig. 30, A) and footpad swelling was determined 48 hours after the last immunization. In Fig 33 values obtained are plotted for the groups under study, and a clear, significant immune response is obtained for LL10 group. These results evidence that live administration of L. lactis LL10 strains elicits a similar response to the control group immunized with purified TS and the ISCOMATRIX adjuvant.

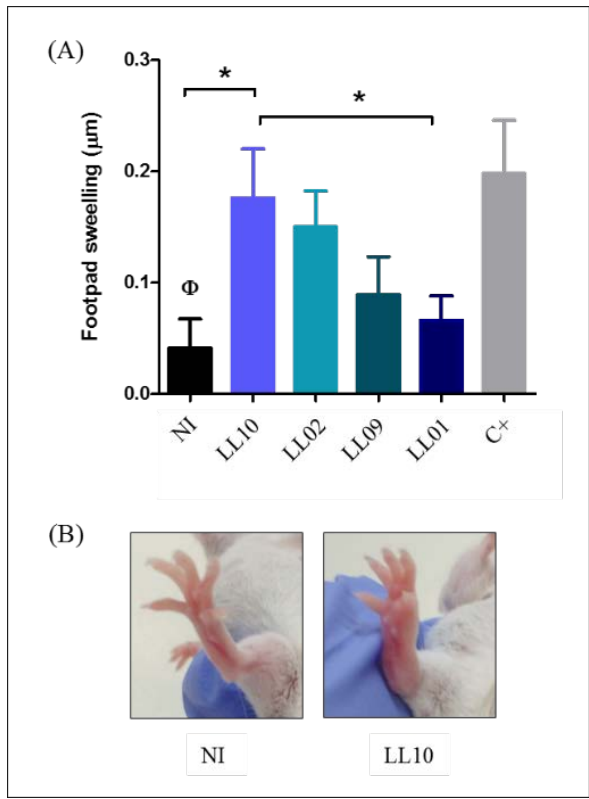

Fig 33 Immune response to $L$. lactis LL10 strain. The immunization protocol as shown in Fig. 30, A was again used to evaluate the response of strain L. lactis LL10, coproducing TScf antigen and c-di-AMP adjuvant at the same time. A: Footpad swelling is significantly higher for this group than for NI and control group LL01. B: Footpad swelling developed by LL10 group. NI: Non-immunized. 


\subsubsection{Conclusions}

In this chapter, an engineered L. lactis strain was constructed to express tscf gene coding a heterologous, immunogenic trans-sialidase (TS) derived peptide (LL02). TScf peptide condenses important $\mathrm{T}$ and $\mathrm{B}$ epitopes, and Western blot analyses confirmed its production. Furthermore, mice oral inoculation with a combination of strains LL02 and LL09 (derived from strain LL03, obtained in the previous chapter) elicited a clear immune response. This encouraging results lead to the development of strain L. lactis LL10, which co-produces in the cytosol the c-di-AMP adjuvant and the optimized TScf antigen.

BALB/c mice oral inoculation with three doses of L. lactis LL10, triggered an immune response evidenced by footpad swelling. A diagram of the LL10-based vaccine explaining the combination of the two expression systems employed is presented in Fig 34. These results show that the engineered strain LL10 could result in an effective vaccine for Chagas disease, which is a serious public health problem in South America.

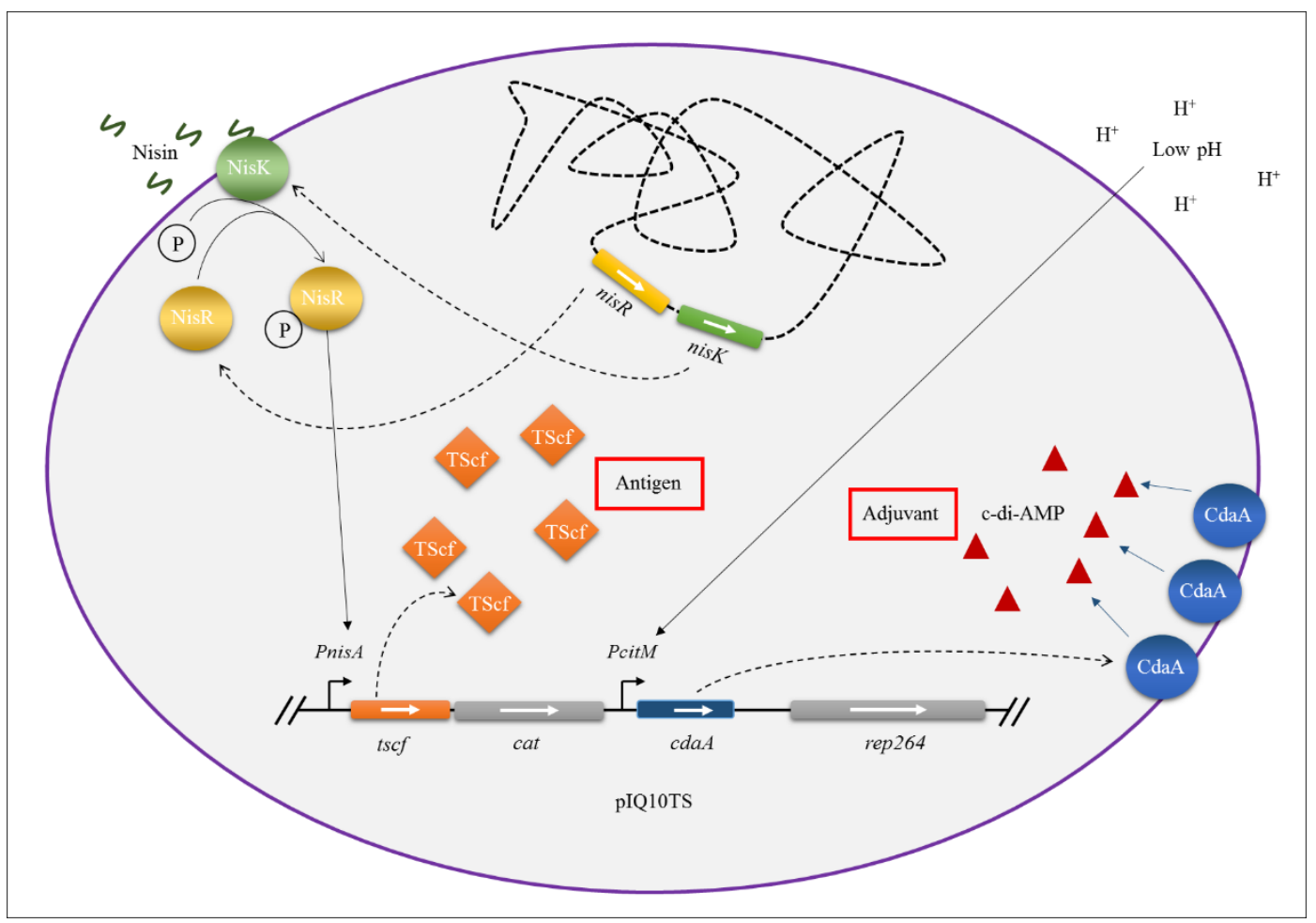

Fig 34 Strain L. lactis LL10: an oral vaccine prototype. Presence of nisRK two-component system in L. lactis genome allows nisin sensing via NisK, upon which NisR is phosphorylated and activates PnisA promoter in pIQ10TS plasmid. On the other hand, low $\mathrm{pH}$ activates in turn PcitM promoter, regulating $c d a A$. CdaA is thus synthesized and c-di-AMP produced. Consequently, both TScf antigen and the adjuvant c-di-AMP are co-produced in the cytosol. 


\subsection{An approach into c-di-AMP metabolism in E. faecalis $\mathrm{JH2}-2$}

\subsubsection{Initial studies into $c d a A$ metabolism}

As in most low GC firmicutes, only one c-di-AMP synthesizing enzyme is found in $E$. faecalis JH2-2 genome. Being a cdaA homologue, it is encoded in the cdaARgImM operon, as expected. Interestingly, glmM gene in the laboratory model strain E. faecalis JH2-2 used in this work, encodes a full 1356 bp open reading frame, whereas the vancomycin-resistant strain V583, presents a shorter version of 1026 bp in its genome, which would result in a 342 amino acids peptide (being the wild type version of 452 amino acids) (Fig 35).

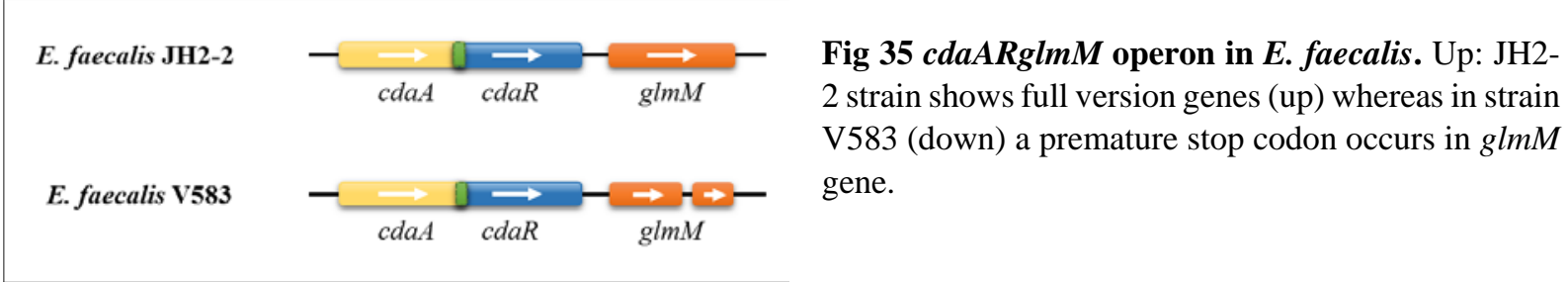

Moreover, a search for genes present in E. faecalis genome coding for proteins with high homology for c-di-AMP degrading enzymes, revealed the presence of a $g d p P$ as well as a pgpH homologue (Fig 36). Interestingly, the cytosolic version of the DHH family of phosphodiesterases was also found encoded in E. faecalis JH2-2 genome. As previously mentioned, the NrnA homologue in B. subtilis is not capable of degrading c-di-AMP, but the corresponding protein named Pde2 in S. pneumoniae possesses c-di-AMP phosphodiesterase activity, and it was proved that Pde1 (a GdpP homologue) as well as Pde2 affect bacterial growth and virulence (68). In conclusion, E. faecalis could count on three c-di-AMP degrading enzymes, and c-di-AMP metabolism in this species could be also related to virulence.

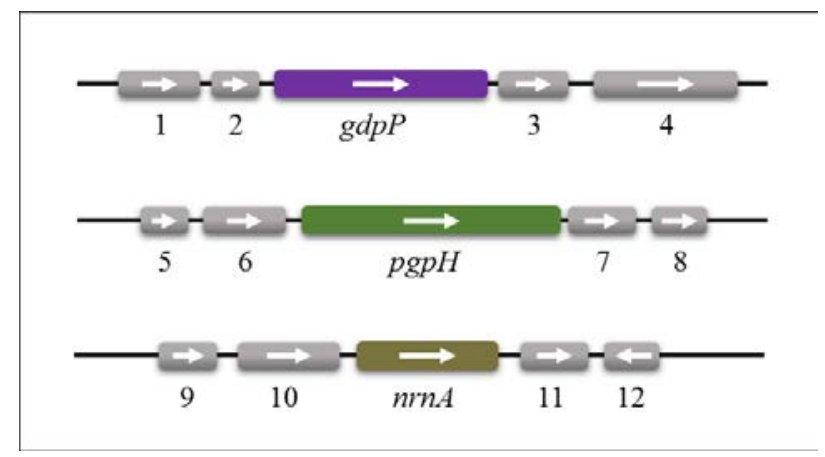

Fig 36 Phosphodiesterases encoded in E. faecalis JH2-2 genome. 1: DNA binding protein. 2: SSU ribosomal protein S18p. 3: LSU ribosomal protein L9p. 4: dnaB. 5: gatB. 6: phoH. 7: metal-dependent hydrolase ybeY. 8: diacylglycerol kinase. 9: metaldependent hydrolase. 10: CBS containing protein. 11: phnA. 12: hypothetical protein 
In the first chapter of this thesis, L. lactis Kup proteins were identified as c-di-AMP interaction partners. A search for genes coding for Kup homologues was therefore performed in E. faecalis JH2-2, which revealed one copy of kup in its genome (Fig 37). Moreover, the presence of other potassium transporters was as well considered in this species. Neither Kdp, nor Ktr homologues were found, but two genes coding for putative members of the Trk potassium transporter family were found. These proteins are predicted as TrkA and TrkH and their genetic contexts are detailed in Fig 37. Interestingly, a homologue to KimA, a novel potassium transporter recently described, was also found encoded in E. faecalis JH2-2 genome (49).

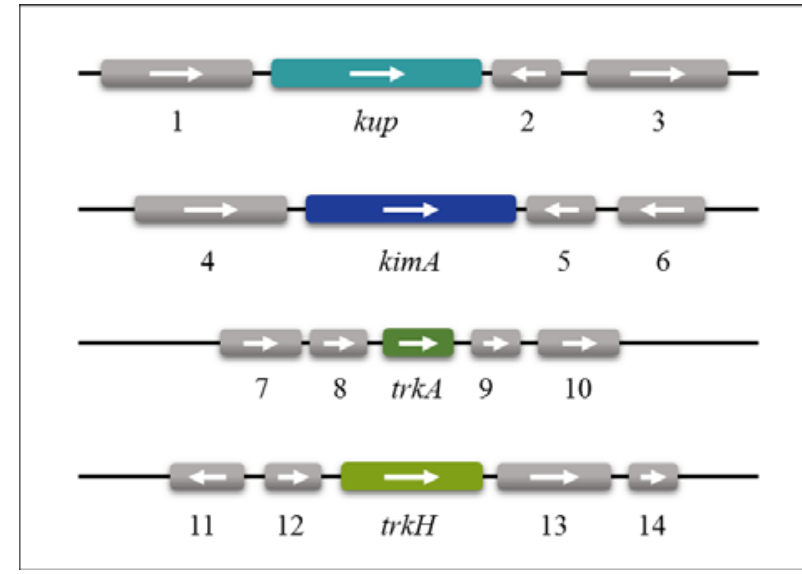

Fig 37 Putative potassium transporters in $E$. faecalis JH2-2. 1: cation-transporting ATPase. 2: transcriptional regulator. $3: \mathrm{Pb}, \mathrm{Cd}, \mathrm{Zn}$ and $\mathrm{Hg}$ transporting ATPase. 4: Co-Zn-Cd resistance protein. 5: acetyl-transferase. 6: L-carnitine choline ABC transporter. 7: liaS. 8: liaR. 9: hypothetical protein. 10: bactoprenol glucosyl transferase. 11: transcriptional regulator. 12: dihydrofolate reductase. 13: $\mathrm{Na}^{+} / \mathrm{H}^{+}$ antiporter. 14: transcriptional regulator.

At present, no studies about E. faecalis JH2-2 potassium transporters are available. Hence, kup gene was cloned in vector pWH844, similarly to kupA and kupB of L. lactis IL1403 in chapter 1. The resulting pIQ311 plasmid was electroporated in E. coli LB650 and strain E. coli LB13 thus obtained was grown on rich LBG medium to check if a trans complementation with kup gene could restore growth at low potassium concentrations.

As seen in Fig 38, E. coli LB13 strain restores growth on LBG supplemented with 10 $\mathrm{mM} \mathrm{KCl}$ under induced conditions. Moreover, this strain behaved similarly to LB09-LB11 strains, harboring trans copies of high affinity potassium uptake systems. This suggests that $E$. faecalis, similarly to L. lactis, counts on the potassium transporter Kup as a high affinity $\mathrm{K}^{+}$ intake system. However, whether this protein, as well as the rest of the putative potassium transporters previously presented interact with c-di-AMP, remains unknown and proposes interesting future investigations. 


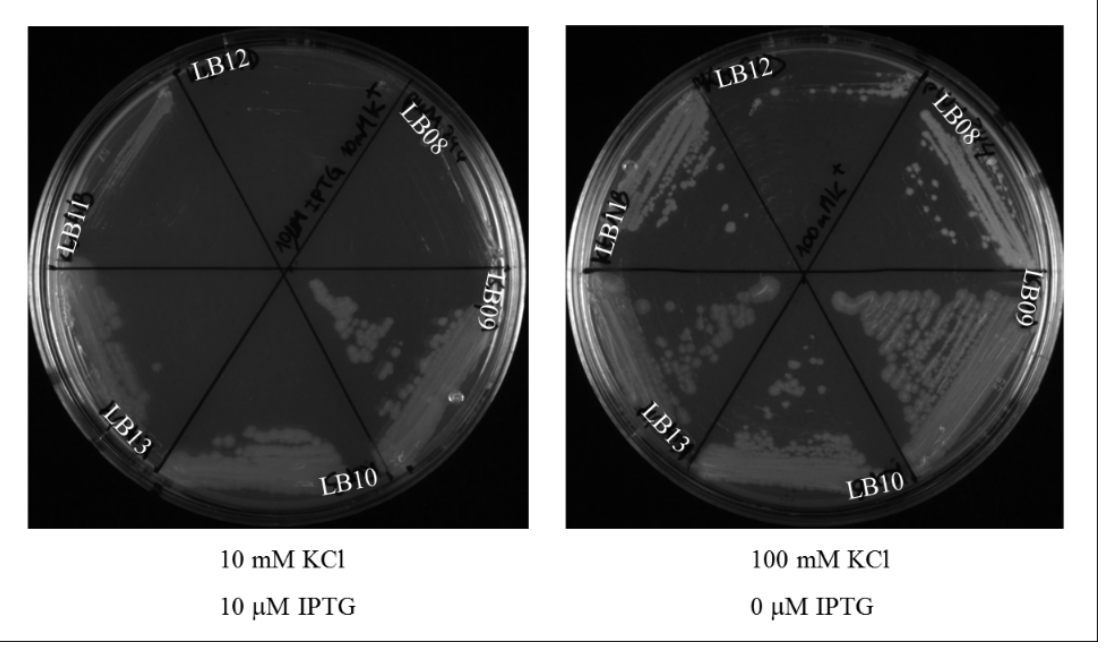

Fig 38 Growth of $\boldsymbol{E}$. coli LB derived strains on LB. LB09 - LB13: E coli LB650 complemented with a trans copy of $k u p A, k u p B, k \operatorname{tr} A / B, k \operatorname{tr} C / D, k u p$, respectively. LB08 contains plasmid pWH844, used as negative control. Strains were plated on LB-agar plates supplemented with $10 \mathrm{mM} \mathrm{KCl}$ and 10 $\mu \mathrm{M}$ IPTG (left) and $100 \mathrm{mM} \mathrm{KCl}$ and no IPTG (right). Low affinity potassium system KtrC/D as well as the negative control with the empty vector only grow at high $\mathrm{K}^{+}$concentrations. Expression of kup genes and $k t r A / B$ system restore growth under low concentrations of $\mathrm{KCl}$.

\subsection{2. $\triangle g d p P$ mutant in $E$. faecalis $\mathrm{JH} 2-2$}

In order to study the response of $\Delta g d p P$ mutation in E. faecalis, deletion by use of the homologous recombination system previously described in section 3.2.1. and Materials and Methods, was performed. Briefly, up and downstream regions of $g d p P$ gene were cloned in the thermosensitive vector pBVGh, and after a series of incubations at the permissive and nonpermissive temperatures, a clean deletion occurs. Antibiotic sensitive colonies are isolated, and mutation is checked via internal and external PCR, as well as sequencing (Fig 15).

After confirming strain $E$. faecalis $\mathrm{JH} 12$ as a $\triangle g d p P$ mutant, a phenotypic analysis was carried out on growth curves performed in rich LBG media, supplemented with different stress factors. As seen in Fig 39, this strain does not show a particular phenotype with respect to JH22 wild type strain when grown in LBG. Nevertheless, in presence of $5.0 \mu \mathrm{g} / \mathrm{ml}$ ampicillin, a hypersentivity phenotype is evidenced. This response was expected, since similar results were obtained for L. lactis $\triangle g d p P$ mutants. 


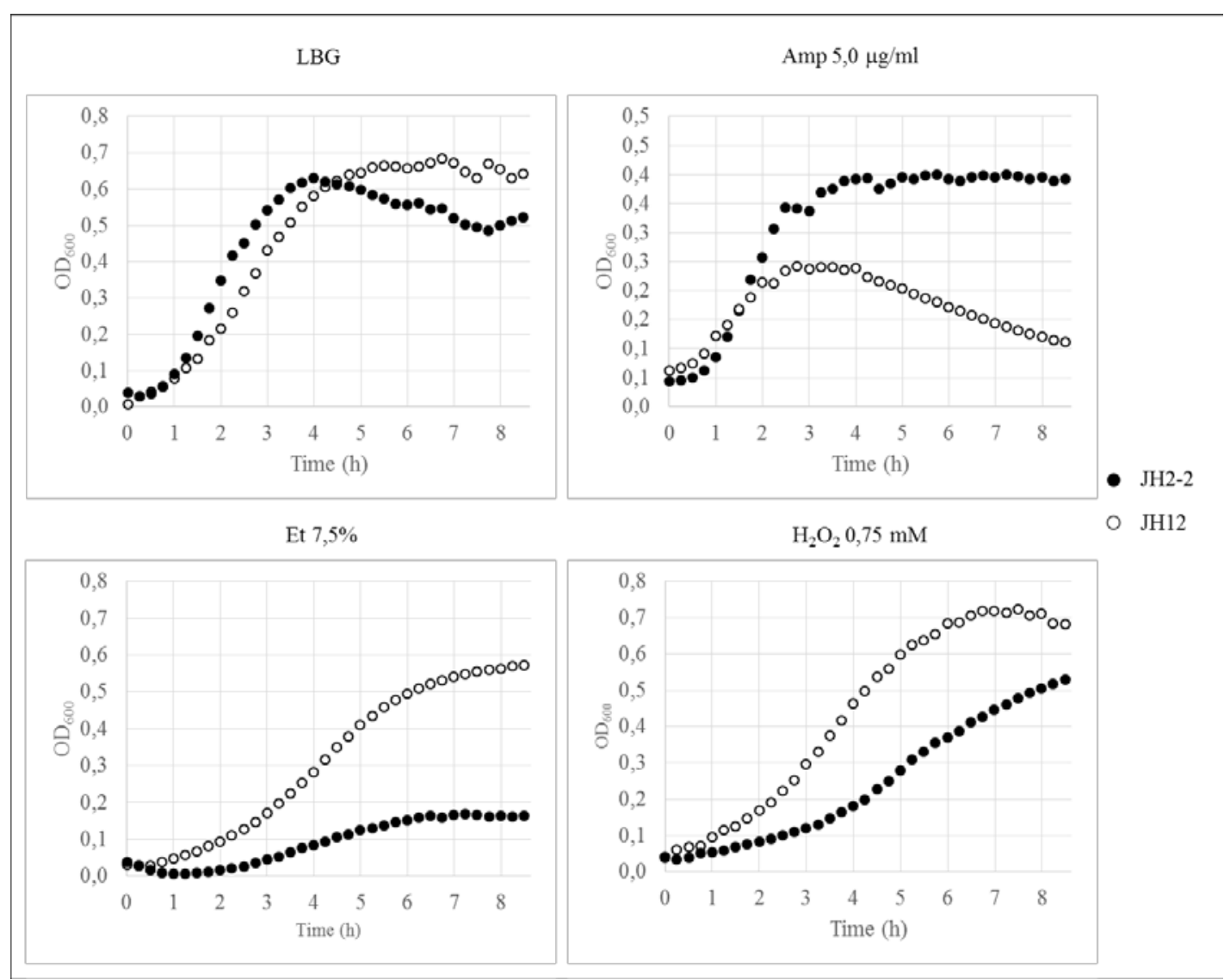

Fig $39 \mathrm{E}$. faecalis JH12 growth in presence of stress factors. Growth curves were performed in rich LBG medium in a microplate reader. Supplements added are indicated above each graph. Similar growth is observed under control LBG conditions. Hypersensitivity phenotype is obtained in presence of $5 \mu \mathrm{g} / \mathrm{ml}$ ampicillin, whereas improved growth was manifested when $7.5 \%$ ethanol and $0.75 \mathrm{mM} \mathrm{H}_{2} \mathrm{O}_{2}$ were added to media. Wild type strain E. faecalis JH2-2 was used as control.

Interestingly, a growth advantage is observed for the mutant strain JH12 when LBG is supplemented with $7.5 \%$ ethanol and $0.75 \mathrm{mM}$ hydrogen peroxide. On the other hand, even though in LB no clear phenotype was observed when $300 \mathrm{mM} \mathrm{KCl}$ was added (not shown), in M17G there was a marked difference, since a hypersensitive phenotype is observed in these conditions (Fig 40). Noteworthy, in M17G control condition, strain JH12 evidences impair growth.

In conclusion, evidence here presented, confirms that in E. faecalis c-di-AMP metabolism affects bacterial response to different stress factors. Altered c-di-AMP levels can confer a growth advantage, but it can also be deleterious for the cell. Due to the diversity of pathways influenced by this second messenger, effects on growth curves experiments are hard to analyze and more specific studies are needed. Even in rich control media LBG and M17G, different phenotypes were observed: the lack of GdpP seems not to affect growth in the former, whereas it results deleterious in the latter.

Lastly, whether Kup, as well as the rest of putative potassium transporters previously presented, interact to c-di-AMP remains unknown and proposes interesting future investigations. 


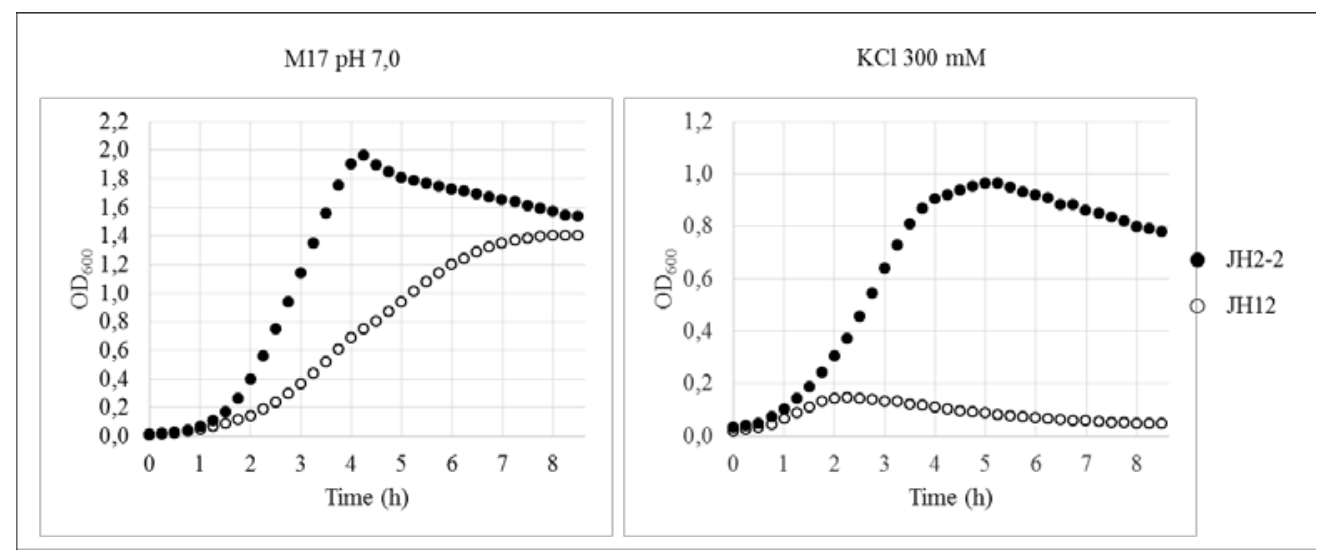

Fig 40 Saline sensitivity in M17G. Growth curves were performed in rich M17G medium in a microplate reader. Addition of $300 \mathrm{mM} \mathrm{KCl}$ results in impaired growth for E. faecalis JH12 (right).

\subsubsection{Virulence analysis of $g d p P$ mutant strain $E$. faecalis $\mathrm{JH} 2-2$ using the greater wax moth Galleria mellonella.}

Historically, the use of invertebrate animals, like the silkworm Bombyx mori and the fruit fly Drosophila melanogaster, has been a very important tool for the development of model microorganisms enabling the study of pathogen-host interactions $(179,180)$. For instance, the development of the greater wax moth Galleria mellonella as an infection model dates from 1938 (181), and due to its adaptability and high proliferation it is a very suitable tool to establish a first approach into the study of pathogenesis and virulence factors $(182,183)$.

Among the most important advantages for the use of $G$. mellonella larvae, easy manipulation, low cost of colony maintenance and its accepted ethical use can be mentioned. It is also of great relevance the fact that these larvae can be grown at $37^{\circ} \mathrm{C}$, temperature at which human pathogens are adapted and therefore synthesis of most virulence factors occur (184). Moreover, and unlike nematode models, which lack phagocytic immune response (185), the insect G. mellonella is capable of eliciting humoral and cellular immune responses. Altogether, G. mellonella is a suitable infection model for the analysis of bacterial virulence factors affecting humans and other mammals (186).

E. faecalis JH12 strain was therefore used to inoculate G. mellonella larvae and analyze the response and possible phenotypic differences due to the lack of the c-di-AMP degrading enzyme, GdpP (see Materials and Methods). Strain JH13 complemented with a trans copy of wild type $g d p P$ gene cloned in vector pBV153 was use to check if, in case of obtaining a distinct phenotype for JH12 mutant strain, complementation occurred. 
Fig 41 shows survival curves obtained after inoculations with 9.10 $10^{6}$ CFU per larvae. Length of horizontal lines along the x-axis corresponds to serial time, or survival duration until a larva dies. Vertical distances between horizontal lines show accumulated probability changes. Censured subjects are shown as black dots.

In the case under study, the first dead individual occurs at approximately 20 hours after inoculation, in all three cases. Nevertheless, at $t=40$ hours, $50 \%$ of larvae still survive for the complemented strain JH13, whereas in JH12 mutant and JH2-2 wild type strains the majority of larvae are already dead. Also, Kaplan-Meier survival analysis indicates a significant differences between the three curves, for which it can be said that the absence of $g d p P$ gene diminishes virulence in E. faecalis, and complementation with vector pIQ104, harboring a wild type copy of $g d p P$, results in an even less virulent strain. Within 50 hours, JH2-2 and JH12 groups are reduced by 90 and 80\%, respectively, while 50\% of JH13 group remain alive.

A negative control was inoculated with PBS solution, which is completely innocuous (not shown).

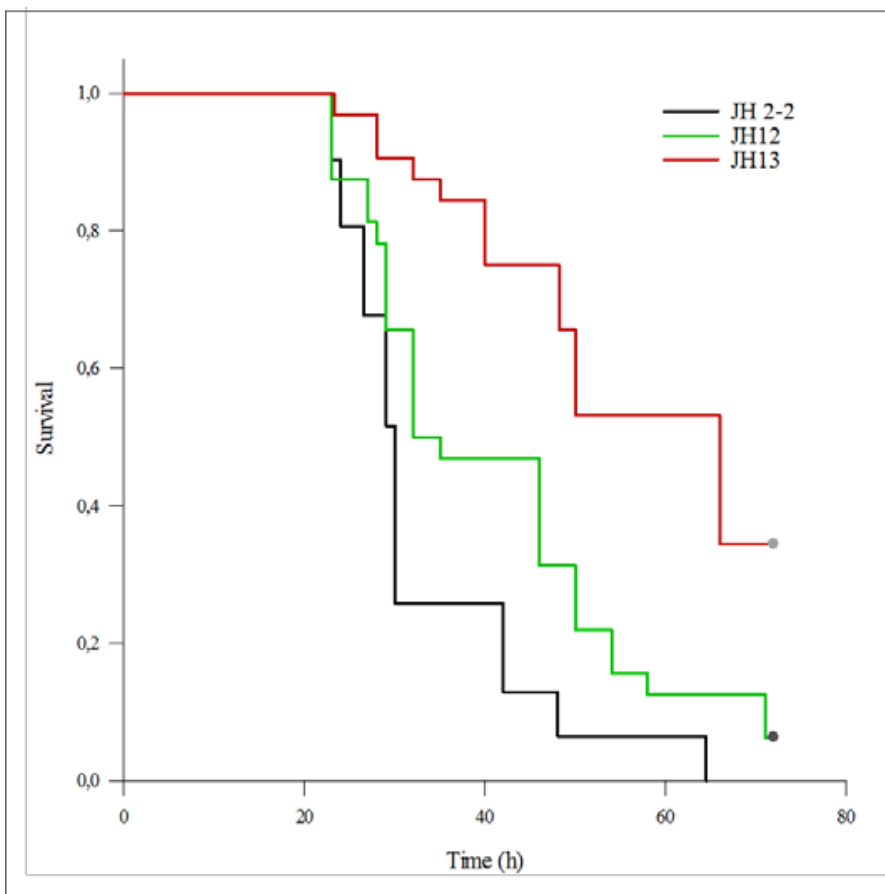

Fig 41 Survival Kaplan-Meier curves for E. faecalis strains under study. $G$. mellonella larvae were inoculated with $9.10^{6} \mathrm{CFU} /$ larvae. E. faecalis JH2-2 (black) was used as control. Green: $\triangle g d p P$ mutant strain E. faecalis JH12. Red: E. faecalis JH13 ( $\triangle g d p P$ complemented in trans with a wild type copy of gdpP gene). 


\subsubsection{Conclusions and Discussion}

In this last chapter, an approach was made into c-di-AMP metabolism in E. faecalis JH2-2. A bioinformatic analysis of genes related to this second messenger shows that glmM could be interrupted in some species of the genus, just as cdaR is in L. lactis (see below).

Furthermore, putative potassium transporters were found encoded in E. faecalis genome. The product of one of them, Kup, was identified as a potassium transporter, and it is likely to have high affinity for this cation like KupA and KupB proteins from L. lactis, previously described.

On the other hand, the analysis of $\triangle g d p P$ on growth curves performed in rich media showed once more for this kind of mutant, a higher susceptibility to ampicillin in LBG and to $\mathrm{KCl}$ in M17G. Noteworthy, growth advantage phenotypes were evidenced in presence of ethanol and $\mathrm{H}_{2} \mathrm{O}_{2}$. Even though a complemented strain was constructed, wild type phenotypes restoration was not achieved (not shown).

Finally, assays in G. mellonella showed lower virulence for $\triangle g d p P$ mutant strain JH12, and even though complemented strain JH13 did not restore wild type phenotype in these experiments either, an even lower virulence was observed. Considering that, as previously mentioned, c-di-AMP levels are tightly regulated, this could be due to the overexpression of gdpP resulting in c-di-AMP concentrations below wild type values. Nevertheless, a clear link between c-di-AMP metabolism and virulence was established for E. faecalis JH2-2. 


\section{General Discussions}

"Science makes people reach selflessly for truth and objectivity; it teaches people to accept reality, with wonder and admiration, not to mention the deep awe and joy that the natural order of things brings to the true scientist" Lise $\mathcal{M}$ eitner 


\subsection{Novel potassium transporters in L. lactis IL1403}

Even though potassium is the most abundant cation in the cell and it is essential for life, no $\mathrm{K}^{+}$transporter systems have so far been described for L. lactis IL1403. In silico research showed different systems encoded in other L. lactis strains, such as Kdp and Ktr systems, both well studied in model microorganisms like B. subtilis and L. monocytogenes. Nevertheless, no kup gene was found in their genomes, and the absence of this system is also verified in S. aureus and S. pneumonia. However, it is widely disseminated in numerous species of Lactobacillus and Enterococcus and it is also present in a few species of Streptococcus and Staphylococcus.

Interestingly, in the L. lactis group, strains with three full copies of the kup gene can be found, although the most represented gene arrangement is the two-copy kupA kupB cluster, as for the case of L. lactis IL1403 presented in this thesis. Nonetheless, some strains only have one copy of the gene, like MG1363 and its derivative NZ9000, the strain of choice for genetic engineering in L. lactis. In these cases, a spontaneous mutation in kupB originates a stop codon, confirming that only one copy of the gene would be enough to regulate potassium uptake and satisfy the vital metabolic needs of the cell concerning cell turgor, proton motive force, and others.

In the Gram-negative model bacterium E. coli, Trchounian and Kobayashi determined in 1999 that the main function of the only Kup in this bacterium (previously known as TrkD) is to uptake $\mathrm{K}^{+}$in hyperosmotic conditions at low $\mathrm{pH}$, being a constitutive, low affinity and low rate system (47). Moreover, it is also the case for Gram - bacteria that different amounts of kup gene copies can be found in the same genome. In Legionella pneumophila, the causal agent of legionelosis, three genes with high similarity to E. coli kup gene were found and named kupA, kupB and kupC (187).

As previously mentioned, topology prediction showed that conserved residues are concentrated at the $\mathrm{N}$-terminal end, which was expected since the cytosolic C-terminal end was proved to be non-essential for potassium translocation, whereas truncation of the protein in the loop between membrane regions 10 and 11 resulted in a non-functional peptide (158). Provided E. coli has no c-di-AMP synthesizing enzymes and consequently potassium transporters are not expected to bind the second messenger in this bacterium, it is reasonable to expect that the cdi-AMP binding site in L. lactis has been acquired at the C-terminal cytosolic half of the protein. 


\subsection{An updated overview of c-di-AMP in L. lactis IL1403}

Even though it is well known that synthesis and degradation of c-di-AMP occurs through specific cyclases and phosphodiesterases (CdaA and GdpP respectively) regulation of CdaA by CdaR is still not exactly understood in L. lactis. Zhu et al. have suggested that CdaR could be a negative regulator, due to experiments carried out in E. coli with different combinations of the $c d a A$ operon fragments, although further investigations are needed to confirm these data (82). In the same work, they report that strain MG1363 has a nucleotide deletion in $c d a R$ gene, leading to a premature stop codon, and, consequently, to a shorter version of $c d a R$, which is actually annotated as a pseudogene. The resulting peptide would be 96 amino acids long, considerably shorter than the wild type 320 amino acid protein. They also state that no other $L$. lactis strain shows this mutation.

Nonetheless, it seems to be also the case for L. lactis IL1403, where a different mutation is found, resulting in a second shorter version of $c d a R$, which would encode a 194 amino acid peptide. From the currently sequenced and assembled 35 L. lactis genomes available in the Genbank database, no other L. lactis strain shows this unusual genotype, i.e. excluding strains IL1403, MG1363 and its derivative NZ9000, the remaining 32 have the typical gene arrangement, which is represented in Fig 42 by the one present in SK11 strain.

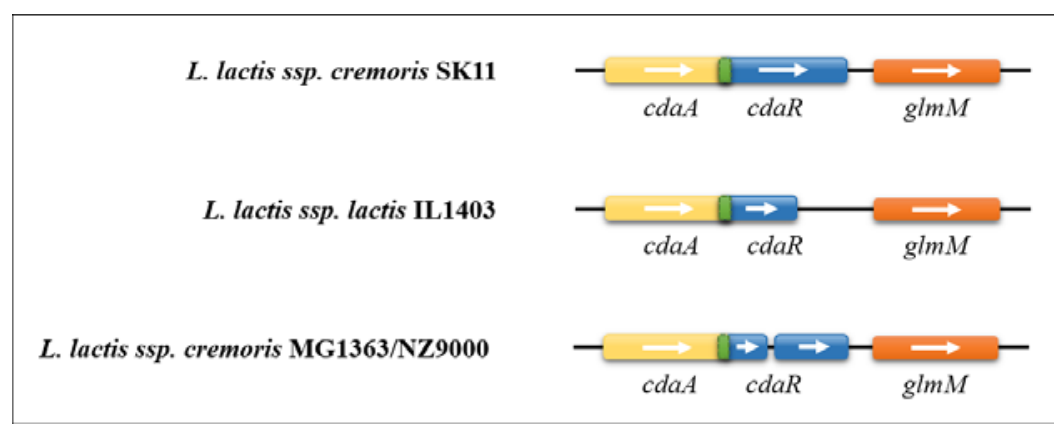

Fig 42 cdaARgImM operon in L. lactis. Three variations of $c d a R$ gene are found among the $35 \mathrm{~L}$. lactis sequenced and assembled strains available in the Genbank database.

To discard the possibility that this different version of cdaR gene could be due to an annotation error, the whole cdaARgImM operon was sequenced, using genomic DNA and primers JN373, JN374, JN385, JN386, JN387, JN388 (see Table XVII). Sequences were assembled and alignment to the theoretical operon available in the Genbank database, allowed confirmation of the premature stop codon. 
So far, only one c-di-AMP degrading enzyme was reported in L. lactis, GdpP. In this work, levels of c-di-AMP in $\triangle g d p P$ are maintained close to wild type ones, suggesting that there could be another enzyme in charge of this metabolite hydrolysis. YheB, a S. pneumonia Pde2 homologue is therefore proposed, and even though its phosphodiesterase activity was confirmed via the BNPP assay, further more specific studies are needed to confirm if it can degrade c-di-AMP.

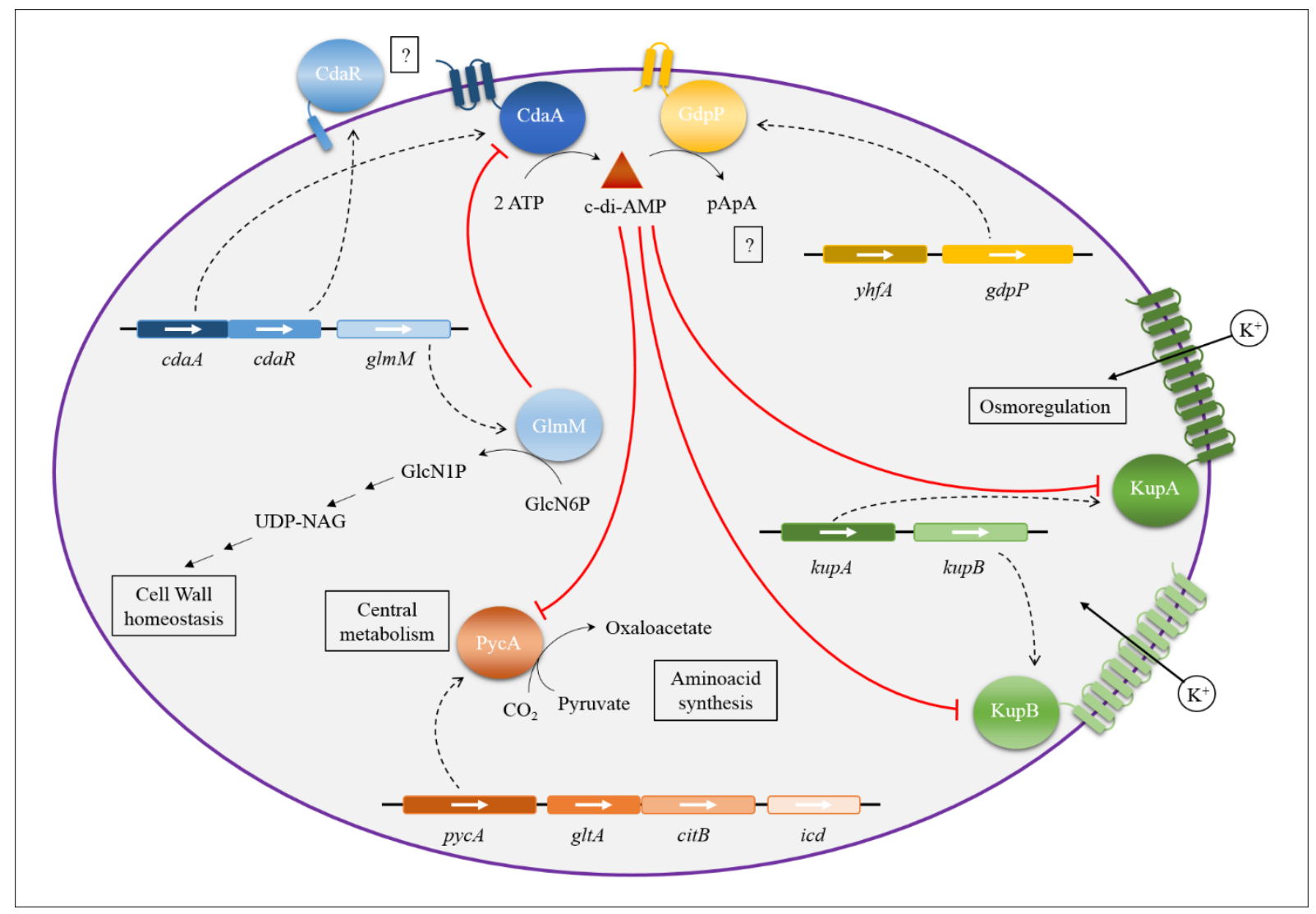

Fig 43. Schematic representation of pathways currently proved to be influenced by c-di-AMP in $L$. Iactis IL1403. Red lines represent inhibitory effect. Black curved arrows indicate synthesis of metabolites. Black dashed arrows stand for transcription and translation of indicated genes to obtain the resulting protein. Question marks denote the lack of data to assess the modulation of CdaA by CdaR and of the stringent response metabolite (p)ppGpp by pApA, which has been proved in other model bacteria to modulate the c-di-AMP degrading enzyme GdpP.

Moreover, it is nowadays well known that degradation of c-di-AMP yields phosphoadenylyl phosphoadenosine (pApA) (Fig 43). This metabolite intervenes in turn in the stringent response, a survival pathway activated under carbon source deprivation, although its specific role and its connection to the stringent response metabolite (p)ppGpp which has been proven to modulate GdpP activity in other model organisms (71), remains unknown in L. lactis. 
The first confirmed modulator of c-di-AMP levels in L. lactis IL1403 was GlmM, negatively modulates CdaA. In this way, c-di-AMP metabolism was first linked to cell wall homeostasis in L. lactis. Moreover, c-di-AMP was recently proved to be involved in central metabolism in L. lactis IL1403 due to the negative regulation it exerts on PycA, the pyruvate carboxylase enzyme in charge of oxaloacetate synthesis (81). This is the first metabolite entering the tricarboxylic acid cycle for $\mathrm{CO}_{2}$ fixation. Nevertheless, it is important to mention that due to the incomplete Krebs cycle present in L. lactis, the main impact of pyruvate carboxylase enzyme is thought to be involved in amino acids synthesis, since oxaloacetate is an aspartate precursor.

Finally, Fig 43 also depicts the novel role of c-di-AMP in L. lactis osmoregulation via inhibition of KupA and KupB, identified and described in this thesis. Since other potassium transporters are present in different strains of L. lactis, the question remains whether all these systems are also regulated by c-di-AMP, as KupA and KupB were proved to be.

\subsection{L. lactis + c-di-AMP: a novel vaccine delivery system}

Vaccination is one of the most important inventions in the field of public health. As described in the introduction, the first vaccines were obtained based on the use of attenuated or inactivated microorganisms. More recently, molecular techniques opened the possibility to develop vaccines using purified fragments of proteins and recombinant antigens. Even though one of the main advantages of this technology are the fewer risks for the patients, they usually show poor immunogenic properties, making the use of adjuvants necessary to potentiate the specific immune response. In addition, most of the currently used vaccines are administered via parenteral routes, and only a few examples of vaccines administered through mucous membranes are commercially available. Since several pathogens enter their hosts through mucosal surfaces, the development of innovative mucosal vaccines is a challenging paradigm.

L. lactis is a versatile bacterium, which counts on decades of experimental research as well as industrial applications ((188), also developed in the introduction). It is a good candidate when considering the delivery of biologically active immunomodulatory proteins or the production of active biological compounds. Very importantly, the safety of L. lactis is well established, having GRAS and QPS status by the Food and Drugs Administration (U.S.A.) and the European Food Safety Authority, respectively. Hence, this microorganism offers a substantial potential as a delivery vector system for vaccines, particularly because it can be 
administrated by diverse mucosal routes like oral, nasal or intravaginal, and it survives passage through GIT as well (189).

In this thesis, L. lactis LL02 was constructed, allowing overproduction of c-di-AMP more than 15 times above wild type levels. Thus, this tool was combined with the nisin inducible expression system widely study in L. lactis to develop strain L. lactis LL10. This is a single novel bacterium designed to deliver the TScf antigen adjuvanted by c-di-AMP in the cytosol. Three successive oral immunizations with this prototype of L. lactis live vaccine elicited a clear anti-TS cellular response, indicating that oral formulations with this microorganism could be used as delivery of diverse heterologous antigens adjuvanted by c-diAMP in order to trigger specific immune protection.

The co-existence of both molecules in the same strain of L. lactis, not only may favor the development of a specific immune response (by exposing immunocompetent cells to both molecules at the same time), but also may help to reduce the cost vaccination programs in developing countries. Moreover, this prototype of L. lactis overproducing c-di-AMP can be combined with other antigens, avoiding expensive and laborious procedures for c-di-AMP production, and enabling systematic research of a variety of antigens. 
5. References

"Science knows no country, Gecause knowledge belongs to humanity, and is the torch which ifluminates the world" Louis Pasteur 
1. Corsetti A, Perpetuini G, Schirone M, Tofalo R, \& Suzzi G (2012) Application of starter cultures to table olive fermentation: an overview on the experimental studies. Frontiers in Microbiology 3:248.

2. Seixas FN, Rios EA, Martinez de Oliveira AL, Beloti V, \& Poveda JM (2018) Selection of Leuconostoc strains isolated from artisanal Serrano Catarinense cheese for use as adjuncts in cheese manufacture. J Sci Food Agric 98(10):3899-3906.

3. Bancalari E, et al. (2017) An integrated strategy to discover Lactobacillus casei group strains for their potential use as aromatic starters. Food Res Int 100(Pt 1):682-690.

4. Liu W, Pang H, Zhang H, \& Cai Y (2014) Biodiversity of Lactic Acid Bacteria. Lactic Acid Bacteria: Fundamentals and Practice, eds Zhang H \& Cai Y (Springer Netherlands, Dordrecht), pp 103-203.

5. Tagg JR, Dajani AS, \& Wannamaker LW (1976) Bacteriocins of gram-positive bacteria. Bacteriol Rev 40(3):722-756.

6. Rilla N, Martinez B, Delgado T, \& Rodriguez A (2003) Inhibition of Clostridium tyrobutyricum in Vidiago cheese by Lactococcus lactis ssp. lactis IPLA 729, a nisin Z producer. Int J Food Microbiol 85(1-2):23-33.

7. De Vuyst L \& Vandamme EJ (1994) Nisin, a lantibiotic produced by Lactococcus lactis subsp. lactis: properties, biosynthesis, fermentation and applications. Bacteriocins of Lactic Acid Bacteria: Microbiology, Genetics and Applications, eds De Vuyst L \& Vandamme EJ (Springer US, Boston, MA), pp 151-221.

8. Sanders ME (2008) Probiotics: Definition, sources, selection, and uses. Clinical Infectious Diseases 46(Supplement_2):S58-S61.

9. Monedero V, Collado MC, \& Rodríguez-Díaz J (2018) Therapeutic opportunities in intestinal microbiota-virus interactions. Trends in Biotechnology 36(7):645-648.

10. Lee H \& Ko G (2016) Antiviral effect of vitamin A on norovirus infection via modulation of the gut microbiome. Sci Rep 6:25835.

11. Techo S, Visessanguan W, Vilaichone RK, \& Tanasupawat S (2018) Characterization and antibacterial activity against helicobacter pylori of Lactic Acid Bacteria isolated from thai fermented rice noodle. Probiotics Antimicrob Proteins:1-11. 
12. Noda M, et al. (2018) The expression of bacteriocin production and self-resistance in Lactobacillus brevis 174A is mediated by two regulatory proteins. Appl Environ Microbiol. doi:10.1128/AEM.02707-17.

13. Noda M, et al. (2015) Characterization and mutational analysis of a two-polypeptide bacteriocin produced by citrus iyo-derived Lactobacillus brevis 174A. Biol Pharm Bull 38(12):1902-1909.

14. Leroy F \& De Vuyst L (2004) Lactic acid bacteria as functional starter cultures for the food fermentation industry. Trends in Food Science \& Technology 15(2):67-78.

15. Bassit N, Boquien CY, Picque D, \& Corrieu G (1993) Effect of initial oxygen concentration on diacetyl and acetoin production by Lactococcus lactis subsp. lactis biovar diacetylactis. Appl Environ Microbiol 59(6):1893-1897.

16. Kelleher P, Bottacini F, Mahony J, Kilcawley KN, \& van Sinderen D (2017) Comparative and functional genomics of the Lactococcus lactis taxon; insights into evolution and niche adaptation. BMC Genomics 18(1):267.

17. Chopin A, Chopin MC, Moillo-Batt A, \& Langella P (1984) Two plasmid-determined restriction and modification systems in Streptococcus lactis. Plasmid 11(3):260-263.

18. Bolotin A, et al. (2001) The complete genome sequence of the lactic acid bacterium Lactococcus lactis ssp. lactis IL1403. Genome Res 11(5):731-753.

19. Gasson MJ (1983) Plasmid complements of Streptococcus lactis NCDO 712 and other lactic streptococci after protoplast-induced curing. J Bacteriol 154(1):1-9.

20. Eichenbaum Z, et al. (1998) Use of the lactococcal nisA promoter to regulate gene expression in gram-positive bacteria: comparison of induction level and promoter strength. Appl Environ Microbiol 64(8):2763-2769.

21. Mierau I \& Kleerebezem M (2005) 10 years of the nisin-controlled gene expression system (NICE) in Lactococcus lactis. Appl Microbiol Biotechnol 68(6):705-717.

22. Linares DM, Kok J, \& Poolman B (2010) Genome sequences of Lactococcus lactis MG1363 (revised) and NZ9000 and comparative physiological studies. J Bacteriol 192(21):5806-5812. 
23. Cortes-Perez NG, et al. (2006) Construction and characterization of a Lactococcus lactis strain deficient in intracellular ClpP and extracellular HtrA proteases. Microbiology 152(Pt 9):2611-2618.

24. Fisher K \& Phillips C (2009) The ecology, epidemiology and virulence of Enterococcus. Microbiology 155(Pt 6):1749-1757.

25. Boehm AB \& Sassoubre LM (2014) Enterococci as indicators of environmental fecal contamination. Enterococci: From Commensals to Leading Causes of Drug Resistant Infection, eds Gilmore MS, Clewell DB, Ike Y, \& Shankar NBoston. https://www.ncbi.nlm.nih.gov/books/NBK190427/

26. Giraffa G (2002) Enterococci from foods. FEMS Microbiol Rev 26(2):163-171.

27. Edmond MB, et al. (1995) Vancomycin-resistant Enterococcus faecium bacteremia: risk factors for infection. Clin Infect Dis 20(5):1126-1133.

28. Abriouel H, et al. (2008) Comparative analysis of genetic diversity and incidence of virulence factors and antibiotic resistance among enterococcal populations from raw fruit and vegetable foods, water and soil, and clinical samples. Int J Food Microbiol 123(1-2):38-49.

29. Centeno JA, Menendez S, Hermida M, \& Rodriguez-Otero JL (1999) Effects of the addition of Enterococcus faecalis in Cebreiro cheese manufacture. Int $J$ Food Microbiol 48(2):97-111.

30. Giraffa G (2003) Functionality of enterococci in dairy products. Int J Food Microbiol 88(2-3):215-222.

31. Nami Y, et al. (2014) A newly isolated probiotic Enterococcus faecalis strain from vagina microbiota enhances apoptosis of human cancer cells. J Appl Microbiol 117(2):498-508.

32. Mendoza F, Maqueda M, Galvez A, Martinez-Bueno M, \& Valdivia E (1999) Antilisterial activity of peptide AS-48 and study of changes induced in the cell envelope properties of an AS-48-adapted strain of Listeria monocytogenes. Appl Environ Microbiol 65(2):618-625.

33. Abriouel H, Valdivia E, Galvez A, \& Maqueda M (1998) Response of Salmonella choleraesuis LT2 spheroplasts and permeabilized cells to the bacteriocin AS-48. Appl Environ Microbiol 64(11):4623-4626. 
34. Khalkhali S \& Mojgani N (2017) Bacteriocinogenic potential and virulence traits of Enterococcus faecium and E. faecalis isolated from human milk. Iran J Microbiol 9(4):224233.

35. Miskin R, Zamir A, \& Elson D (1970) Inactivation and reactivation of ribosomal subunits: the peptidyl transferase activity of the 50 s subunit of Escherihia coli. J Mol Biol 54(2):355-378.

36. Zamir A, Miskin R, \& Elson D (1971) Inactivation and reactivation of ribosomal subunits: amino acyl-transfer RNA binding activity of the 30 s subunit of Escherichia coli. $J$ Mol Biol 60(2):347-364.

37. Ukai H, Matsuzawa H, Ito K, Yamada M, \& Nishimura A (1998) ftsE(Ts) affects translocation of $\mathrm{K}+$-pump proteins into the cytoplasmic membrane of Escherichia coli. $\mathrm{J}$ Bacteriol 180(14):3663-3670.

38. Follmann M, et al. (2009) Potassium transport in Corynebacterium glutamicum is facilitated by the putative channel protein CglK, which is essential for $\mathrm{pH}$ homeostasis and growth at acidic pH. J Bacteriol 191(9):2944-2952.

39. Krulwich TA, Sachs G, \& Padan E (2011) Molecular aspects of bacterial pH sensing and homeostasis. Nat Rev Microbiol 9(5):330-343.

40. Epstein W (2003) The roles and regulation of potassium in bacteria. Prog Nucleic Acid Res Mol Biol 75:293-320.

41. Radchenko MV, et al. (2006) Potassium/proton antiport system of Escherichia coli. $J$ Biol Chem 281(29):19822-19829.

42. Brown AD (1990) Microbial water stress physiology. Principles and perspectives (John Wiley \& Sons, Chichester) p xiii + 313 pp.

43. Roberts MF (2005) Organic compatible solutes of halotolerant and halophilic microorganisms. Saline Systems 1:5.

44. Trchounian A \& Kobayashi H (2000) K+ uptake by fermenting Escherichia coli cells: pH dependent mode of the TrkA system operating. Biosci Rep 20(4):277-288.

45. Epstein W (2016) The KdpD Sensor Kinase of Escherichia coli Responds to several distinct signals to turn on expression of the Kdp transport system. J Bacteriol 198(2):212-220. 
46. Ballal A, Basu B, \& Apte SK (2007) The Kdp-ATPase system and its regulation. $J$ Biosci 32(3):559-568.

47. Trchounian A \& Kobayashi H (1999) Kup is the major $\mathrm{K}+$ uptake system in Escherichia coli upon hyper-osmotic stress at a low pH. FEBS Lett 447(2-3):144-148.

48. Holtmann G, Bakker EP, Uozumi N, \& Bremer E (2003) KtrAB and KtrCD: two K+ uptake systems in Bacillus subtilis and their role in adaptation to hypertonicity. $J$ Bacteriol 185(4):1289-1298.

49. Gundlach J, et al. (2017) Control of potassium homeostasis is an essential function of the second messenger cyclic di-AMP in Bacillus subtilis. Sci Signal 10(475).

50. Corrigan RM, et al. (2013) Systematic identification of conserved bacterial c-di-AMP receptor proteins. Proc Natl Acad Sci U S A 110(22):9084-9089.

51. Witte G, Hartung S, Buttner K, \& Hopfner KP (2008) Structural biochemistry of a bacterial checkpoint protein reveals diadenylate cyclase activity regulated by DNA recombination intermediates. Mol Cell 30(2):167-178.

52. Witte CE, et al. (2013) Cyclic di-AMP is critical for Listeria monocytogenes growth, cell wall homeostasis, and establishment of infection. MBio 4(3):e00282-00213.

53. Oppenheimer-Shaanan Y, Wexselblatt E, Katzhendler J, Yavin E, \& Ben-Yehuda S (2011) c-di-AMP reports DNA integrity during sporulation in Bacillus subtilis. EMBO Rep 12(6):594-601.

54. Gundlach J, et al. (2015) An essential poison: synthesis and degradation of cyclic diAMP in Bacillus subtilis. J Bacteriol 197(20):3265-3274.

55. Woodward JJ, Iavarone AT, \& Portnoy DA (2010) c-di-AMP secreted by intracellular Listeria monocytogenes activates a host type I interferon response. Science 328(5986):17031705.

56. Cron LE, et al. (2011) Two DHH subfamily 1 proteins contribute to pneumococcal virulence and confer protection against pneumococcal disease. Infect Immun 79(9):3697-3710.

57. Huynh TN, et al. (2015) An HD-domain phosphodiesterase mediates cooperative hydrolysis of c-di-AMP to affect bacterial growth and virulence. Proc Natl Acad Sci U S A 112(7):E747-756. 
58. Griffiths JM \& O'Neill AJ (2012) Loss of function of the gdpP protein leads to joint beta-lactam/glycopeptide tolerance in Staphylococcus aureus. Antimicrob Agents Chemother 56(1):579-581.

59. Luo Y \& Helmann JD (2012) Analysis of the role of Bacillus subtilis sigma(M) in betalactam resistance reveals an essential role for c-di-AMP in peptidoglycan homeostasis. $\mathrm{Mol}$ Microbiol 83(3):623-639.

60. Whiteley AT, Pollock AJ, \& Portnoy DA (2015) The PAMP c-di-AMP is essential for Listeria monocytogenes growth in rich but not minimal media due to a toxic increase in (p)ppGpp. [corrected]. Cell Host Microbe 17(6):788-798.

61. Moscoso JA, et al. (2015) Binding of cyclic di-AMP to the Staphylococcus aureus sensor kinase KdpD occurs via the universal stress protein domain and downregulates the expression of the Kdp potassium transporter. J Bacteriol 198(1):98-110.

62. Zeden MS, et al. (2018) Cyclic di-adenosine monophosphate (c-di-AMP) is required for osmotic regulation in Staphylococcus aureus but dispensable for viability in anaerobic conditions. J Biol Chem 293(9):3180-3200.

63. Bai Y, et al. (2012) Mycobacterium tuberculosis Rv3586 (DacA) is a diadenylate cyclase that converts ATP or ADP into c-di-AMP. PLoS One 7(4):e35206.

64. Corrigan RM \& Grundling A (2013) Cyclic di-AMP: another second messenger enters the fray. Nat Rev Microbiol 11(8):513-524.

65. Opoku-Temeng C \& Sintim HO (2016) Potent inhibition of cyclic diadenylate monophosphate cyclase by the antiparasitic drug, suramin. Chem Commun (Camb) 52(19):3754-3757.

66. Taylor BL \& Zhulin IB (1999) PAS domains: internal sensors of oxygen, redox potential, and light. Microbiol Mol Biol Rev 63(2):479-506.

67. Aravind L \& Koonin EV (1998) The HD domain defines a new superfamily of metaldependent phosphohydrolases. Trends Biochem Sci 23(12):469-472.

68. Bai Y, et al. (2013) Two DHH subfamily 1 proteins in Streptococcus pneumoniae possess cyclic di-AMP phosphodiesterase activity and affect bacterial growth and virulence. $J$ Bacteriol 195(22):5123-5132. 
69. Ye M, et al. (2014) DhhP, a cyclic di-AMP phosphodiesterase of Borrelia burgdorferi, is essential for cell growth and virulence. Infect Immun 82(5):1840-1849.

70. Manikandan K, et al. (2014) Two-step synthesis and hydrolysis of cyclic di-AMP in Mycobacterium tuberculosis. PLoS One 9(1):e86096.

71. Corrigan RM, Bowman L, Willis AR, Kaever V, \& Grundling A (2015) Cross-talk between two nucleotide-signaling pathways in Staphylococcus aureus. J Biol Chem 290(9):5826-5839.

72. Rao F, et al. (2010) YybT is a signaling protein that contains a cyclic dinucleotide phosphodiesterase domain and a GGDEF domain with ATPase activity. J Biol Chem 285(1):473-482.

73. Huynh TN \& Woodward JJ (2016) Too much of a good thing: regulated depletion of cdi-AMP in the bacterial cytoplasm. Curr Opin Microbiol 30:22-29.

74. Smith WM, et al. (2012) Heat resistance and salt hypersensitivity in Lactococcus lactis due to spontaneous mutation of llmg_1816 (gdpP) induced by high-temperature growth. Appl Environ Microbiol 78(21):7753-7759.

75. Corrigan RM, Abbott JC, Burhenne H, Kaever V, \& Grundling A (2011) c-di-AMP is a new second messenger in Staphylococcus aureus with a role in controlling cell size and envelope stress. PLoS Pathog 7(9):e1002217.

76. Zhang L, Li W, \& He ZG (2013) DarR, a TetR-like transcriptional factor, is a cyclic diAMP-responsive repressor in Mycobacterium smegmatis. J Biol Chem 288(5):3085-3096.

77. Bai Y, et al. (2014) Cyclic di-AMP impairs potassium uptake mediated by a cyclic diAMP binding protein in Streptococcus pneumoniae. J Bacteriol 196(3):614-623.

78. Chin KH, et al. (2015) Structural insights into the distinct binding mode of cyclic diAMP with SaCpaA_RCK. Biochemistry 54(31):4936-4951.

79. Muller M, Hopfner KP, \& Witte G (2015) c-di-AMP recognition by Staphylococcus aureus PstA. FEBS Lett 589(1):45-51.

80. Schuster CF, et al. (2016) The second messenger c-di-AMP inhibits the osmolyte uptake system OpuC in Staphylococcus aureus. Sci Signal 9(441):ra81. 
81. Sureka K, et al. (2014) The cyclic dinucleotide c-di-AMP is an allosteric regulator of metabolic enzyme function. Cell 158(6):1389-1401.

82. Zhu Y, et al. (2016) Cyclic-di-AMP synthesis by the diadenylate cyclase CdaA is modulated by the peptidoglycan biosynthesis enzyme GlmM in Lactococcus lactis. Mol Microbiol 99(6):1015-1027.

83. Rallu F, Gruss A, Ehrlich SD, \& Maguin E (2000) Acid- and multistress-resistant mutants of Lactococcus lactis: identification of intracellular stress signals. Mol Microbiol 35(3):517-528.

84. Rao F, Ji Q, Soehano I, \& Liang ZX (2011) Unusual heme-binding PAS domain from YybT family proteins. J Bacteriol 193(7):1543-1551.

85. Tan E, et al. (2013) Solution structure of the PAS domain of a thermophilic YybT protein homolog reveals a potential ligand-binding site. J Biol Chem 288(17):11949-11959.

86. Choi PH, et al. (2017) Structural and functional studies of pyruvate carboxylase regulation by cyclic di-AMP in lactic acid bacteria. Proc Natl Acad Sci U S A 114(35):E7226E7235.

87. Kamegaya T, Kuroda K, \& Hayakawa Y (2011) Identification of a Streptococcus pyogenes SF370 gene involved in production of c-di-AMP. Nagoya J Med Sci 73(1-2):49-57.

88. Du B, et al. (2014) Functional analysis of c-di-AMP phosphodiesterase, GdpP, in Streptococcus suis serotype 2. Microbiol Res 169(9-10):749-758.

89. Du B \& Sun JH (2015) Diadenylate cyclase evaluation of ssDacA (SSU98_1483) in Streptococcus suis serotype 2. Genet Mol Res 14(2):6917-6924.

90. Cho KH \& Kang SO (2013) Streptococcus pyogenes c-di-AMP phosphodiesterase, GdpP, influences SpeB processing and virulence. PLoS One 8(7):e69425.

91. Underwood AJ, Zhang Y, Metzger DW, \& Bai G (2014) Detection of cyclic di-AMP using a competitive ELISA with a unique pneumococcal cyclic di-AMP binding protein. $J$ Microbiol Methods 107:58-62.

92. Cheng X, et al. (2016) Regulation of oxidative response and extracellular polysaccharide synthesis by a diadenylate cyclase in Streptococcus mutans. Environ Microbiol 18(3):904-922. 
93. Peng X, Michalek S, \& Wu H (2016) Effects of diadenylate cyclase deficiency on synthesis of extracellular polysaccharide matrix of Streptococcus mutans revisit. Environ Microbiol 18(11):3612-3619.

94. Peng X, Zhang Y, Bai G, Zhou X, \& Wu H (2016) Cyclic di-AMP mediates biofilm formation. Mol Microbiol 99(5):945-959.

95. Andrade WA, et al. (2016) Group B Streptococcus degrades cyclic-di-AMP to modulate STING-dependent type I interferon production. Cell Host Microbe 20(1):49-59.

96. Wang X, et al. (2017) A novel phosphodiesterase of the GdpP family modulates cyclic di-AMP levels in response to cell membrane stress in daptomycin-resistant Enterococci. Antimicrob Agents Chemother 61(3).

97. Sales Junior PA, et al. (2017) Experimental and clinical treatment of chagas disease: a review. Am J Trop Med Hyg 97(5):1289-1303.

98. Dias JC (2007) Southern Cone Initiative for the elimination of domestic populations of Triatoma infestans and the interruption of transfusional Chagas disease. Historical aspects, present situation, and perspectives. Mem Inst Oswaldo Cruz 102 Suppl 1:11-18.

99. Thakare R, Dasgupta A, \& Chopra S (2018) An update on benznidazole for the treatment of patients with Chagas disease. Drugs Today (Barc) 54(1):15-23.

100. Olivera MJ, Fory JA, \& Olivera AJ (2017) Therapeutic drug monitoring of benznidazole and nifurtimox: a systematic review and quality assessment of published clinical practice guidelines. Rev Soc Bras Med Trop 50(6):748-755.

101. Schenkman S, Jiang MS, Hart GW, \& Nussenzweig V (1991) A novel cell surface transsialidase of Trypanosoma cruzi generates a stage-specific epitope required for invasion of mammalian cells. Cell 65(7):1117-1125.

102. Ming M, Chuenkova M, Ortega-Barria E, \& Pereira ME (1993) Mediation of Trypanosoma cruzi invasion by sialic acid on the host cell and trans-sialidase on the trypanosome. Mol Biochem Parasitol 59(2):243-252.

103. Villalta F, Smith CM, Ruiz-Ruano A, \& Lima MF (2001) A ligand that Trypanosoma cruzi uses to bind to mammalian cells to initiate infection. FEBS Lett 505(3):383-388. 
104. Schenkman S, Eichinger D, Pereira ME, \& Nussenzweig V (1994) Structural and functional properties of Trypanosoma trans-sialidase. Annu Rev Microbiol 48:499-523.

105. Dumonteil E, Escobedo-Ortegon J, Reyes-Rodriguez N, Arjona-Torres A, \& RamirezSierra MJ (2004) Immunotherapy of Trypanosoma cruzi infection with DNA vaccines in mice. Infect Immun 72(1):46-53.

106. Martinez-Campos V, et al. (2015) Expression, purification, immunogenicity, and protective efficacy of a recombinant Tc24 antigen as a vaccine against Trypanosoma cruzi infection in mice. Vaccine 33(36):4505-4512.

107. Villanueva-Lizama LE, et al. (2018) Trypanosoma cruzi vaccine candidate antigens Tc24 and TSA-1 recall memory immune response associated with HLA-A and -B supertypes in Chagasic chronic patients from Mexico. PLoS Negl Trop Dis 12(1):e0006240.

108. Riedel S (2005) Edward Jenner and the history of smallpox and vaccination. Proc (Bayl Univ Med Cent) 18(1):21-25.

109. Lopez AL, Gonzales MLA, Aldaba JG, \& Nair GB (2014) Killed oral cholera vaccines: history, development and implementation challenges. Therapeutic Advances in Vaccines 2(5):123-136.

110. Koury R \& Warrington SJ (2017) Rabies. StatPearls, Treasure Island (FL)). https://www.ncbi.nlm.nih.gov/books/NBK448076/

111. Pliaka V, Kyriakopoulou Z, \& Markoulatos P (2012) Risks associated with the use of live-attenuated vaccine poliovirus strains and the strategies for control and eradication of paralytic poliomyelitis. Expert Rev Vaccines 11(5):609-628.

112. Baxter D (2007) Active and passive immunity, vaccine types, excipients and licensing. Occupational Medicine 57(8):552-556.

113. Clark Z \& Maurer DM (2018) Pneumococcal vaccines in chronic obstructive pulmonary disease. Am Fam Physician 97(7):463-464.

114. Gupta RK \& Siber GR (1995) Adjuvants for human vaccines--current status, problems and future prospects. Vaccine 13(14):1263-1276.

115. Klijn N, Weerkamp AH, \& de Vos WM (1995) Genetic marking of Lactococcus lactis shows its survival in the human gastrointestinal tract. Appl Environ Microbiol 61(7):2771-2774. 
116. Steidler L, et al. (2000) Treatment of murine colitis by Lactococcus lactis secreting interleukin-10. Science 289(5483):1352-1355.

117. Iwaki M, et al. (1990) Oral immunization with recombinant Streptococcus lactis carrying the Streptococcus mutans surface protein antigen gene. Infect Immun 58(9):29292934.

118. Braat $\mathrm{H}$, et al. (2006) A phase I trial with transgenic bacteria expressing interleukin-10 in Crohn's disease. Clin Gastroenterol Hepatol 4(6):754-759.

119. Vandenbroucke K, et al. (2010) Orally administered L. lactis secreting an anti-TNF nanobody demonstrate efficacy in chronic colitis. Mucosal Immunol 3(1):49-56.

120. Wells JM, Wilson PW, Norton PM, Gasson MJ, \& Le Page RW (1993) Lactococcus lactis: high-level expression of tetanus toxin fragment $\mathrm{C}$ and protection against lethal challenge. Mol Microbiol 8(6):1155-1162.

121. Saez D, Fernandez P, Rivera A, Andrews E, \& Onate A (2012) Oral immunization of mice with recombinant Lactococcus lactis expressing $\mathrm{Cu}, \mathrm{Zn}$ superoxide dismutase of Brucella abortus triggers protective immunity. Vaccine 30(7):1283-1290.

122. Asensi GF, et al. (2013) Oral immunization with Lactococcus lactis secreting attenuated recombinant staphylococcal enterotoxin B induces a protective immune response in a murine model. Microb Cell Fact 12:32.

123. Ahmed B, Loos M, Vanrompay D, \& Cox E (2013) Mucosal priming of the murine immune system against enterohemorrhagic Escherichia coli O157:H7 using Lactococcus lactis expressing the type III secretion system protein EspB. Vet Immunol Immunopathol 152(12):141-145.

124. Cauchard S, et al. (2011) Mucosal co-immunization of mice with recombinant lactococci secreting VapA antigen and leptin elicits a protective immune response against Rhodococcus equi infection. Vaccine 30(1):95-102.

125. Marelli B, Perez AR, Banchio C, de Mendoza D, \& Magni C (2011) Oral immunization with live Lactococcus lactis expressing rotavirus VP8 subunit induces specific immune response in mice. $J$ Virol Methods 175(1):28-37. 
126. Bermudez-Humaran LG, et al. (2005) A novel mucosal vaccine based on live Lactococci expressing E7 antigen and IL-12 induces systemic and mucosal immune responses and protects mice against human papillomavirus type 16-induced tumors. J Immunol 175(11):7297-7302.

127. Cano-Garrido O, Seras-Franzoso J, \& Garcia-Fruitos E (2015) Lactic acid bacteria: reviewing the potential of a promising delivery live vector for biomedical purposes. Microb 1 .

Lasztity R (2009) Food Quality And Standards - Volume III (EOLSS Publishers Company Limited).

128. Pashine A, Valiante NM, \& Ulmer JB (2005) Targeting the innate immune response with improved vaccine adjuvants. Nat Med 11(4 Suppl):S63-68.

129. Apost, et al. (2016) Adjuvants: classification, modus operandi, and licensing. journal of immunology Research 2016:16.

130. Ebensen T, et al. (2011) Bis-(3',5')-cyclic dimeric adenosine monophosphate: strong Th1/Th2/Th17 promoting mucosal adjuvant. Vaccine 29(32):5210-5220.

131. Rueckert C, et al. (2017) Cyclic dinucleotides modulate induced type I IFN responses in innate immune cells by degradation of STING. FASEB J 31(7):3107-3115.

132. Matos MN, et al. (2017) Immunization with Tc52 or its amino terminal domain adjuvanted with c-di-AMP induces Th17+Th1 specific immune responses and confers protection against Trypanosoma cruzi. PLoS Negl Trop Dis 11(2):e0005300.

133. Landi A, et al. (2017) Superior immunogenicity of HCV envelope glycoproteins when adjuvanted with cyclic-di-AMP, a STING activator or archaeosomes. Vaccine 35(50):69496956.

134. Ebensen T, et al. (2017) Mucosal administration of cycle-di-nucleotide-adjuvanted virosomes efficiently induces protection against Influenza H5N1 in mice. Front Immunol 8:1223.

135. Weiss W, Weiland F, \& Gorg A (2009) Protein detection and quantitation technologies for gel-based proteome analysis. Methods Mol Biol 564:59-82.

136. Efron B (1988) Logistic regression, Survival Analysis, and the Kaplan-Meier curve. 83(402):414-425. 
137. Bertona D, et al. (2017) Development and assessment of a new cage-like particle adjuvant. J Pharm Pharmacol 69(10):1293-1303.

138. Commichau FM, Gibhardt J, Halbedel S, Gundlach J, \& Stulke J (2017) A delicate connection: c-di-AMP affects cell integrity by controlling osmolyte transport. Trends Microbiol. VOLUME 26, ISSUE 3, P175-185

139. Commichau FM, Dickmanns A, Gundlach J, Ficner R, \& Stulke J (2015) A jack of all trades: the multiple roles of the unique essential second messenger cyclic di-AMP. Mol Microbiol 97(2):189-204.

140. Gundlach J, Commichau FM, \& Stulke J (2017) Perspective of ions and messengers: an intricate link between potassium, glutamate, and cyclic di-AMP. Curr Genet. Volume 64, Issue 1, pp 191-195

141. Quintero FJ \& Blatt MR (1997) A new family of K+ transporters from Arabidopsis that are conserved across phyla. FEBS Lett 415(2):206-211.

142. Sato Y, et al. (2014) Defining membrane spanning domains and crucial membranelocalized acidic amino acid residues for $\mathrm{K}(+)$ transport of a Kup/HAK/KT-type Escherichia coli potassium transporter. J Biochem 155(5):315-323.

143. Jiang Y, Pico A, Cadene M, Chait BT, \& MacKinnon R (2001) Structure of the RCK domain from the $\mathrm{E}$. coli $\mathrm{K}+$ channel and demonstration of its presence in the human $\mathrm{BK}$ channel. Neuron 29(3):593-601.

144. Schlosser A, Meldorf M, Stumpe S, Bakker EP, \& Epstein W (1995) TrkH and its homolog, TrkG, determine the specificity and kinetics of cation transport by the Trk system of Escherichia coli. J Bacteriol 177(7):1908-1910.

145. Williams T, Bauer S, Beier D, \& Kuhn M (2005) Construction and characterization of Listeria monocytogenes mutants with in-frame deletions in the response regulator genes identified in the genome sequence. Infect Immun 73(5):3152-3159.

146. Cocaign-Bousquet M, Garrigues C, Novak L, Lindley ND, \& Loublere P (1995) Rational development of a simple synthetic medium for the sustained growth of Lactococcus lactis. Journal of Applied Bacteriology 79(1):108-116. 
147. Zhang G, Mills DA, \& Block DE (2009) Development of chemically defined media supporting high-cell-density growth of lactococci, enterococci, and streptococci. Appl Environ Microbiol 75(4):1080-1087.

148. Marelli B \& Magni C (2009) A simple expression system for Lactococcus lactis and Enterococcus faecalis. World J Microbiol Biotechnol 26(6):999-1007.

149. Blancato VS \& Magni C (2010) A chimeric vector for efficient chromosomal modification in Enterococcus faecalis and other lactic acid bacteria. Lett Appl Microbiol 50(5):542-546.

150. Waxman DJ \& Strominger JL (1983) Penicillin-binding proteins and the mechanism of action of beta-lactam antibiotics. Annu Rev Biochem 52:825-869.

151. Hubschwerlen C (2007) 7.17 - $\beta$-Lactam Antibiotics A2 - Taylor, John B. Comprehensive Medicinal Chemistry II, ed Triggle DJ (Elsevier, Oxford), pp 479-518.

152. Watanakunakorn C (1984) Mode of action and in-vitro activity of vancomycin. $J$ Antimicrob Chemother 14 Suppl D:7-18.

153. Salton MR (1958) The lysis of micro-organisms by lysozyme and related enzymes. $J$ Gen Microbiol 18(2):481-490.

154. Rismondo J, et al. (2016) Phenotypes associated with the essential diadenylate cyclase CdaA and its potential regulator CdaR in the human pathogen Listeria monocytogenes. $J$ Bacteriol 198(3):416-426.

155. Yang J, et al. (2014) Deletion of the cyclic di-AMP phosphodiesterase gene (cnpB) in Mycobacterium tuberculosis leads to reduced virulence in a mouse model of infection. Mol Microbiol 93(1):65-79.

156. Mehne FM, et al. (2013) Cyclic di-AMP homeostasis in Bacillus subtilis: both lack and high level accumulation of the nucleotide are detrimental for cell growth. J Biol Chem 288(3):2004-2017.

157. Freire-de-Lima L, Fonseca LM, Oeltmann T, Mendonca-Previato L, \& Previato JO (2015) The trans-sialidase, the major Trypanosoma cruzi virulence factor: Three decades of studies. Glycobiology 25(11):1142-1149. 
158. Bontempi IA, et al. (2015) Efficacy of a trans-sialidase-ISCOMATRIX subunit vaccine candidate to protect against experimental Chagas disease. Vaccine 33(10):1274-1283.

159. Bontempi I, et al. (2017) Trans-sialidase overcomes many antigens to be used as a vaccine candidate against Trypanosoma cruzi. Immunotherapy 9(7):555-565.

160. Rosenberg CS, Martin DL, \& Tarleton RL (2010) CD8+ T cells specific for immunodominant trans-sialidase epitopes contribute to control of Trypanosoma cruzi infection but are not required for resistance. J Immunol 185(1):560-568.

161. Eickhoff CS, et al. (2011) Co-administration of a plasmid DNA encoding IL-15 improves long-term protection of a genetic vaccine against Trypanosoma cruzi. PLoS Negl Trop Dis 5(3):e983.

162. Alvarez P, Leguizamon MS, Buscaglia CA, Pitcovsky TA, \& Campetella O (2001) Multiple overlapping epitopes in the repetitive unit of the shed acute-phase antigen from Trypanosoma cruzi enhance its immunogenic properties. Infect Immun 69(12):7946-7949.

163. Matsumoto Y, Ishii M, Shimizu K, Kawamoto S, \& Sekimizu K (2017) A silkworm infection model to evaluate antifungal drugs for Cryptococcosis. Med Mycol J 58(4):E131E137.

164. Mondotte JA \& Saleh MC (2018) Antiviral immune response and the route of infection in Drosophila melanogaster. Adv Virus Res 100:247-278.

165. Smith TL (1938) Genetical Studies on the wax moth Galleria mellonella Linn. Genetics 23(1):115-137.

166. Rakic Martinez M, Wiedmann M, Ferguson M, \& Datta AR (2017) Assessment of Listeria monocytogenes virulence in the Galleria mellonella insect larvae model. PLoS One 12(9):e0184557.

167. Meir M, Grosfeld T, \& Barkan D (2018) Establishment and validation of Galleria mellonella as a novel model organism to study Mycobacterium abscessus infection, pathogenesis, and treatment. Antimicrob Agents Chemother 62(4).

168. Cook SM \& McArthur JD (2013) Developing Galleria mellonella as a model host for human pathogens. Virulence 4(5):350-353. 
169. Ermolaeva MA \& Schumacher B (2014) Insights from the worm: the C. elegans model for innate immunity. Semin Immunol 26(4):303-309.

170. Kavanagh K \& Reeves EP (2004) Exploiting the potential of insects for in vivo pathogenicity testing of microbial pathogens. FEMS Microbiol Rev 28(1):101-112.

171. Hori JI, Pereira MS, Roy CR, Nagai H, \& Zamboni DS (2013) Identification and functional characterization of $\mathrm{K}(+)$ transporters encoded by Legionella pneumophila kup genes. Cell Microbiol 15(12):2006-2019.

172. Song AA, In LLA, Lim SHE, \& Rahim RA (2017) A review on Lactococcus lactis: from food to factory. Microb Cell Fact 16(1):55.

173. Wells JM \& Mercenier A (2008) Mucosal delivery of therapeutic and prophylactic molecules using lactic acid bacteria. Nat Rev Microbiol 6(5):349-362.Cell Fact 14:137.

174. Hamann, A. 1991. PhD thesis. University of Osnabrück, Osnabrück, Federal Republic of Germany.

175. Studier and Moffatt, 1986. Use of bacteriophage T7 RNA polymerase to direct selective high-level expression of cloned genes. J Mol Biol. 1986 May 5;189(1):113-30.

176. Hanahan, 1983. Studies on transformation of Escherichia coli with plasmids. $J$ Mol Biol. 1983 Jun 5;166(4):557-80.

177. Law et al. 1995. A system to generate chromosomal mutations in Lactococcus lactis which allows fast analysis of targeted genes. J Bacteriol. 1995 Dec;177(24):7011-8.

178. Guzman et al. 1995. Tight regulation, modulation, and high-level expression by vectors containing the arabinose PBAD promoter. J Bacteriol. 1995 Jul;177(14):4121-30.

179. De Ruyter et al. 1996. Controlled gene expression systems for Lactococcus lactis with the food-grade inducer nisin. Appl Environ Microbiol. 1996 Oct;62(10):3662-7.

180. Grubek, Z. Ms. Thesis 2016. Georg August Universität, Göttingen, Germany. 
6. Appendix

"Valid criticism does you a favor" Carl Sagan 


\subsection{Peptide identification after c-di-AMP pull-down experiment.}

Table XIV Peptide identification after c-di-AMP pull-down experiment

\begin{tabular}{|c|c|c|c|c|c|}
\hline Identified Protein & Accession Number & $\begin{array}{l}\text { Molecular } \\
\text { Weight }\end{array}$ & + & - & $\begin{array}{l}\text { Ratio } \\
+/-\end{array}$ \\
\hline $\begin{array}{l}\text { DNA polymerase III, subunits beta and } \\
\text { tau }\end{array}$ & Q9CDM5_LACLA & $61 \mathrm{kDa}$ & 34 & $<0.5$ & 69,00 \\
\hline 30S ribosomal protein S6 & RS6_LACLA & $11 \mathrm{kDa}$ & 15 & $<0.5$ & 31,00 \\
\hline Uncharacterized protein & Q9CIR6_LACLA & $62 \mathrm{kDa}$ & 13 & $<0.5$ & 27,00 \\
\hline PpGpp synthetase I & Q9CJ94_LACLA & $84 \mathrm{kDa}$ & 11 & $<0.5$ & 23,00 \\
\hline Protease & Q9CE72_LACLA & $47 \mathrm{kDa}$ & 11 & $<0.5$ & 23,00 \\
\hline Uncharacterized protein & Q9CED1_LACLA & $12 \mathrm{kDa}$ & 11 & $<0.5$ & 23,00 \\
\hline $\begin{array}{l}\text { 3-hydroxy-3-methylglutaryl coenzyme A } \\
\text { reductase }\end{array}$ & Q9CFB1_LACLA & $45 \mathrm{kDa}$ & 10 & $<0.5$ & 21,00 \\
\hline 6,7-dimethyl-8-ribityllumazine synthase & RISB_LACLA & $17 \mathrm{kDa}$ & 10 & $<0.5$ & 21,00 \\
\hline Aspartate racemase & Q9CDJ4_LACLA & $28 \mathrm{kDa}$ & 8 & $<0.5$ & 17,00 \\
\hline Uncharacterized protein & Q9CEX0_LACLA & $22 \mathrm{kDa}$ & 8 & $<0.5$ & 17,00 \\
\hline Initiation-control protein YabA & YABA_LACLA & $13 \mathrm{kDa}$ & 8 & $<0.5$ & 17,00 \\
\hline ATP-dependent DNA helicase RecG & Q9CDH6_LACLA & $75 \mathrm{kDa}$ & 7 & $<0.5$ & 15,00 \\
\hline Pyrimidine-nucleoside phosphorylase & Q9CFM5_LACLA & $47 \mathrm{kDa}$ & 7 & $<0.5$ & 15,00 \\
\hline Oxidoreductase & Q9CEV1_LACLA & $28 \mathrm{kDa}$ & 7 & $<0.5$ & 15,00 \\
\hline Uncharacterized protein & Q9CFC1_LACLA & $21 \mathrm{kDa}$ & 7 & $<0.5$ & 15,00 \\
\hline Uncharacterized protein & Q9CDN8_LACLA & $17 \mathrm{kDa}$ & 7 & $<0.5$ & 15,00 \\
\hline $\begin{array}{l}\text { Single-stranded DNA specific } \\
\text { exonuclease }\end{array}$ & Q9CHT6_LACLA & $84 \mathrm{kDa}$ & 6 & $<0.5$ & 13,00 \\
\hline $\begin{array}{l}\text { Glutamine } \mathrm{ABC} \text { transporter permease and } \\
\text { substrate binding protein }\end{array}$ & Q9CES5_LACLA & $78 \mathrm{kDa}$ & 6 & $<0.5$ & 13,00 \\
\hline Peptide chain release factor 2 & RF2_LACLA & $42 \mathrm{kDa}$ & 6 & $<0.5$ & 13,00 \\
\hline Uncharacterized protein & Q9CHI4_LACLA & $10 \mathrm{kDa}$ & 6 & $<0.5$ & 13,00 \\
\hline Cell-division ATP-binding protein FtsE & Q9CGX0_LACLA & $26 \mathrm{kDa}$ & 6 & $<0.5$ & 13,00 \\
\hline DNA repair protein RecN & Q9CH78_LACLA & $63 \mathrm{kDa}$ & 34 & 3 & 9,86 \\
\hline Uncharacterized protein & Q9CFB8_LACLA & $15 \mathrm{kDa}$ & 45 & 5 & 8,27 \\
\hline Aspartate--tRNA ligase & SYD_LACLA & $67 \mathrm{kDa}$ & 164 & 20 & 8,02 \\
\hline Uncharacterized protein & Q9CEI1_LACLA & $13 \mathrm{kDa}$ & 26 & 3 & 7,57 \\
\hline DNA mismatch repair protein MutL & MUTL_LACLA & $74 \mathrm{kDa}$ & 30 & 4 & 6,78 \\
\hline $\begin{array}{l}\text { Glutamine--fructose-6-phosphate } \\
\text { aminotransferase [isomerizing] }\end{array}$ & GLMS_LACLA & $66 \mathrm{kDa}$ & 206 & 31 & 6,56 \\
\hline
\end{tabular}


Phosphoenolpyruvate-protein

phosphotransferase

Septation ring formation regulator EzrA

NADH oxidase

Elongation factor 4

ABC transporter ATP binding protein

Uncharacterized protein

$\mathrm{N}$-acetylmuramidase

Universal stress protein

Arginine--tRNA ligase

Folylpolyglutamate synthase

Homoserine dehydrogenase

Uncharacterized protein

Hydroxymethylglutaryl-CoA synthase

Gamma-glutamyl phosphate reductase

Sugar ABC transporter ATP binding

protein

$\mathrm{ABC}$ transporter ATP binding protein

Glucose-6-phosphate isomerase

Peptide-binding protein

Uncharacterized protein

Peptide chain release factor 3

ABC transporter ATP binding protein

Chaperone protein DnaK

Lysine--tRNA ligase

Probable phosphoketolase

Uncharacterized protein

Uncharacterized protein

Peptide chain release factor 1

Uncharacterized protein

UPF0145 protein YjfJ

Signal recognition particle receptor FtsY

Uncharacterized protein

Polysaccharide biosynthesis protein

UDP-N-acetylglucosamine 1-

carboxyvinyltransferase 2

\begin{tabular}{|c|c|c|c|}
\hline PT1_LACLA & $63 \mathrm{kDa}$ & 126 & 19 \\
\hline EZRA_LACLA & $66 \mathrm{kDa}$ & 35 & 5 \\
\hline Q9CIG9_LACLA & $49 \mathrm{kDa}$ & 38 & 6 \\
\hline LEPA_LACLA & $68 \mathrm{kDa}$ & 32 & 5 \\
\hline Q9CJA0_LACLA & $58 \mathrm{kDa}$ & 61 & 10 \\
\hline Q9CGM2_LACLA & $14 \mathrm{kDa}$ & 14 & 2 \\
\hline Q9CED5_LACLA & $50 \mathrm{kDa}$ & 14 & 2 \\
\hline Q9CEI3_LACLA & $16 \mathrm{kDa}$ & 120 & 21 \\
\hline SYR_LACLA & $63 \mathrm{kDa}$ & 30 & 5 \\
\hline Q9CGE0_LACLA & $48 \mathrm{kDa}$ & 12 & 2 \\
\hline DHOM_LACLA & $47 \mathrm{kDa}$ & 21 & 4 \\
\hline Q9CGJ5_LACLA & $19 \mathrm{kDa}$ & 11 & 2 \\
\hline Q9CFA9_LACLA & $43 \mathrm{kDa}$ & 11 & 2 \\
\hline PROA_LACLA & $45 \mathrm{kDa}$ & 24 & 5 \\
\hline Q9CFY3_LACLA & $56 \mathrm{kDa}$ & 15 & 3 \\
\hline Q9CFC6_LACLA & $70 \mathrm{kDa}$ & 31 & 7 \\
\hline G6PI_LACLA & $50 \mathrm{kDa}$ & 10 & 2 \\
\hline Q9CI27_LACLA & $60 \mathrm{kDa}$ & 14 & 3 \\
\hline Q9CJ15_LACLA & $13 \mathrm{kDa}$ & 18 & 4 \\
\hline RF3_LACLA & $60 \mathrm{kDa}$ & 30 & 7 \\
\hline Q9CJC5_LACLA & $61 \mathrm{kDa}$ & 62 & 15 \\
\hline DNAK_LACLA & $65 \mathrm{kDa}$ & 150 & 37 \\
\hline SYK_LACLA & $57 \mathrm{kDa}$ & 21 & 5 \\
\hline PHK_LACLA & $93 \mathrm{kDa}$ & 9 & 2 \\
\hline Q9CDZ4_LACLA & $18 \mathrm{kDa}$ & 43 & 11 \\
\hline Q9CID7_LACLA & $17 \mathrm{kDa}$ & 68 & 18 \\
\hline RF1_LACLA & $40 \mathrm{kDa}$ & 27 & 7 \\
\hline Q9CFU3_LACLA & $15 \mathrm{kDa}$ & 16 & 4 \\
\hline YJFJ_LACLA & $12 \mathrm{kDa}$ & 30 & 8 \\
\hline Q9CHB9_LACLA & $51 \mathrm{kDa}$ & 392 & 109 \\
\hline Q9CF71_LACLA & $56 \mathrm{kDa}$ & 12 & 3 \\
\hline Q9CIZ9_LACLA & $70 \mathrm{kDa}$ & 12 & 3 \\
\hline MURA2_LACLA & $45 \mathrm{kDa}$ & 12 & 3 \\
\hline
\end{tabular}


Aromatic amino acid specific

aminotransferase

S-adenosylmethionine synthase

Glycosyltransferase

Uncharacterized protein

Asparagine synthetase

Probable serine/threonine-protein kinase

Adenylosuccinate synthetase

NifU protein

ATP-dependent protease ATP-binding

subunit

Nicotinate phosphoribosyltransferase

Segregation and condensation protein B

50S ribosomal protein L5

Uncharacterized protein

Uncharacterized protein

Flotillin-like protein

Cell division protein ftsA

Uncharacterized protein

Transcriptional regulator

Tagatose-6-phosphate kinase

Alpha-subunit L-serine dehydratase

Uncharacterized protein

Probable nicotinate-nucleotide

adenylyltransferase

Uncharacterized protein

Lipoprotein

Uncharacterized protein

tRNA uridine 5-

carboxymethylaminomethyl modification enzyme

ATP-dependent Clp protease ATP-

binding subunit

ATP synthase subunit delta

Endonuclease MutS2

30S ribosomal protein S4

\begin{tabular}{|c|c|c|c|}
\hline Q9CJE0_LACLA & $43 \mathrm{kDa}$ & 60 & 17 \\
\hline METK_LACLA & $43 \mathrm{kDa}$ & 170 & 49 \\
\hline Q9CJ00_LACLA & $38 \mathrm{kDa}$ & 15 & 4 \\
\hline Q9CDS4_LACLA & $9 \mathrm{kDa}$ & 15 & 4 \\
\hline Q9CDJ2_LACLA & $72 \mathrm{kDa}$ & 32 & 9 \\
\hline PKNB_LACLA & $68 \mathrm{kDa}$ & 8 & 2 \\
\hline PURA_LACLA & $47 \mathrm{kDa}$ & 143 & 43 \\
\hline Q9CEP7_LACLA & $13 \mathrm{kDa}$ & 11 & 3 \\
\hline Q9CHS9_LACLA & $90 \mathrm{kDa}$ & 66 & 20 \\
\hline Q9CGJ6_LACLA & $56 \mathrm{kDa}$ & 82 & 25 \\
\hline SCPB_LACLA & $21 \mathrm{kDa}$ & 27 & 8 \\
\hline RL5_LACLA & $20 \mathrm{kDa}$ & 14 & 4 \\
\hline Q9CDS3_LACLA & $37 \mathrm{kDa}$ & 36 & 11 \\
\hline Q9CJ16_LACLA & $58 \mathrm{kDa}$ & 155 & 50 \\
\hline Q9CHJ2_LACLA & $54 \mathrm{kDa}$ & 41 & 13 \\
\hline Q9CEH1_LACLA & $49 \mathrm{kDa}$ & 91 & 30 \\
\hline Q9CF27_LACLA & $38 \mathrm{kDa}$ & 10 & 3 \\
\hline Q9CE68_LACLA & $15 \mathrm{kDa}$ & 10 & 3 \\
\hline Q9CGY4_LACLA & $34 \mathrm{kDa}$ & 7 & 2 \\
\hline Q9CHA7_LACLA & $30 \mathrm{kDa}$ & 7 & 2 \\
\hline Q9CF30_LACLA & $43 \mathrm{kDa}$ & 7 & 2 \\
\hline Q9CIY1_LACLA & $23 \mathrm{kDa}$ & 7 & 2 \\
\hline Q9CF63_LACLA & $64 \mathrm{kDa}$ & 7 & 2 \\
\hline Q9CIN8_LACLA & $31 \mathrm{kDa}$ & 7 & 2 \\
\hline Q9CEF2_LACLA & $18 \mathrm{kDa}$ & 243 & 81 \\
\hline MNMG_LACLA & $69 \mathrm{kDa}$ & 27 & 9 \\
\hline CLPE_LACLA & $83 \mathrm{kDa}$ & 481 & 168 \\
\hline ATPD_LACLA & $19 \mathrm{kDa}$ & 18 & 6 \\
\hline MUTS2_LACLA & $87 \mathrm{kDa}$ & 15 & 5 \\
\hline RS4_LACLA & $23 \mathrm{kDa}$ & 15 & 5 \\
\hline
\end{tabular}


ATP-dependent Clp protease ATP-

binding subunit ClpX

UDP-N-acetylglucosamine--N-

acetylmuramyl-(pentapeptide)

pyrophosphoryl-undecaprenol N-

acetylglucosamine transferase

Uncharacterized protein

Replicative DNA helicase

Uncharacterized protein

Aminopeptidase C

Amino acid aminohydrolase

Mannose-specific PTS system component IID

$60 \mathrm{kDa}$ chaperonin

Prophage pi2 protein 02

Uncharacterized protein

Biotin carboxylase

DNA gyrase subunit A

Asparagine synthetase B

UDP-N-acetylglucosamine 1-

carboxyvinyltransferase 1

UvrABC system protein B

Protein RecA, chromosomal

30S ribosomal protein S10

DNA mismatch repair protein MutS

Diadenosine 5',5"'-P1,P4-tetraphosphate hydrolase

Alpha-acetolactate synthase

Riboflavin biosynthesis protein RibBA

Glutamate--tRNA ligase

Oligopeptide ABC transporter substrate

binding protein

Maltose ABC transporter substrate

binding protein

GTPase HflX

Uncharacterized protein

\begin{tabular}{|c|c|c|c|c|}
\hline CLPX_LACLA & $46 \mathrm{kDa}$ & 270 & 96 & 2,80 \\
\hline MURG_LACLA & $39 \mathrm{kDa}$ & 9 & 3 & 2,71 \\
\hline Q9CEP4_LACLA & $45 \mathrm{kDa}$ & 105 & 39 & 2,67 \\
\hline Q9CHI5_LACLA & $50 \mathrm{kDa}$ & 53 & 20 & 2,61 \\
\hline Q9CGB4_LACLA & $48 \mathrm{kDa}$ & 19 & 7 & 2,60 \\
\hline PEPC_LACLA & $50 \mathrm{kDa}$ & 6 & 2 & 2,60 \\
\hline Q9CGY6_LACLA & $42 \mathrm{kDa}$ & 6 & 2 & 2,60 \\
\hline Q9CEX6_LACLA & $34 \mathrm{kDa}$ & 6 & 2 & 2,60 \\
\hline CH60_LACLA & $57 \mathrm{kDa}$ & 219 & 84 & 2,60 \\
\hline Q9CGT3_LACLA & $21 \mathrm{kDa}$ & 24 & 9 & 2,58 \\
\hline Q9CG71_LACLA & $20 \mathrm{kDa}$ & 29 & 11 & 2,57 \\
\hline Q9CHF3_LACLA & $49 \mathrm{kDa}$ & 52 & 20 & 2,56 \\
\hline Q9CGI5_LACLA & $93 \mathrm{kDa}$ & 11 & 4 & 2,56 \\
\hline Q9CIK5_LACLA & $60 \mathrm{kDa}$ & 205 & 81 & 2,52 \\
\hline MURA1_LACLA & $46 \mathrm{kDa}$ & 94 & 37 & 2,52 \\
\hline UVRB_LACLA & $79 \mathrm{kDa}$ & 76 & 30 & 2,51 \\
\hline RECA_LACLA & $41 \mathrm{kDa}$ & 136 & 54 & 2,50 \\
\hline RS10_LACLA & $12 \mathrm{kDa}$ & 50 & 20 & 2,46 \\
\hline MUTS_LACLA & $94 \mathrm{kDa}$ & 13 & 5 & 2 \\
\hline Q9CI07_LACLA & $18 \mathrm{kDa}$ & 13 & 5 & 2,45 \\
\hline Q7DAV2_LACLA & $61 \mathrm{kDa}$ & 164 & 67 & 2,44 \\
\hline Q9CGU7_LACLA & $44 \mathrm{kDa}$ & 8 & 3 & $2,4 u$ \\
\hline SYE_LACLA & $55 \mathrm{kDa}$ & 8 & 3 & 2,4 \\
\hline Q9CIL2_LACLA & $60 \mathrm{kDa}$ & 117 & 48 & 2,4 \\
\hline Q9CEZ8_LACLA & $44 \mathrm{kDa}$ & 292 & 123 & 2,3 \\
\hline Q9CIY3_LACLA & $43 \mathrm{kDa}$ & 29 & 12 & 2,36 \\
\hline Q9CDF6_LACLA & $22 \mathrm{kDa}$ & 10 & 4 & \\
\hline
\end{tabular}


30S ribosomal protein S7

Nitrogen regulatory protein P-II

GTPase Der

Glycine betaine transport ATP-binding protein OpuAA

Alkyl hydroperoxide reductase

DNA polymerase III subunit beta

Universal stress protein

Formate acetyltransferase

$\mathrm{ABC}$ transporter ATP binding protein

Spermidine/putrescine import ATP-

binding protein PotA

Cysteine--tRNA ligase

Fumarate reductase flavoprotein subunit

Uncharacterized protein

Glucose kinase

Ribonuclease Y

Acetate-SH-citrate lyase ligase

50S ribosomal protein L14

Amino acid ABC transporter substrate

binding protein

Foldase protein PrsA

$\mathrm{NH}(3)$-dependent $\mathrm{NAD}(+)$ synthetase

S-adenosylmethionine:tRNA

ribosyltransferase-isomerase

Thymidylate synthase

Uncharacterized protein

Uncharacterized protein

30S ribosomal protein S5

UDP-N-acetylmuramoylalanine--D-

glutamate ligase

Mannose-specific PTS system component IIAB

Triosephosphate isomerase

Malonyl CoA-acyl carrier protein

transacylase

\begin{tabular}{|c|c|c|c|}
\hline RS7_LACLA & $18 \mathrm{kDa}$ & 10 & 4 \\
\hline Q9CF90_LACLA & $13 \mathrm{kDa}$ & 10 & 4 \\
\hline DER_LACLA & $49 \mathrm{kDa}$ & 63 & 27 \\
\hline OPUAA_LACLA & $46 \mathrm{kDa}$ & 48 & 21 \\
\hline Q9CIL9_LACLA & $21 \mathrm{kDa}$ & 211 & 94 \\
\hline DPO3B_LACLA & $42 \mathrm{kDa}$ & 14 & 6 \\
\hline Q9CH60_LACLA & $16 \mathrm{kDa}$ & 14 & 6 \\
\hline PFL_LACLA & $89 \mathrm{kDa}$ & 36 & 16 \\
\hline Q9CGN4_LACLA & $72 \mathrm{kDa}$ & 18 & 8 \\
\hline POTA_LACLA & $48 \mathrm{kDa}$ & 22 & 10 \\
\hline SYC_LACLA & $51 \mathrm{kDa}$ & 7 & 3 \\
\hline Q9CGH2_LACLA & $53 \mathrm{kDa}$ & 26 & 12 \\
\hline Q9CED6_LACLA & $41 \mathrm{kDa}$ & 81 & 38 \\
\hline Q9CE25_LACLA & $34 \mathrm{kDa}$ & 9 & 4 \\
\hline RNY_LACLA & $60 \mathrm{kDa}$ & 11 & 5 \\
\hline Q9CGB0_LACLA & $39 \mathrm{kDa}$ & 11 & 5 \\
\hline RL14_LACLA & $13 \mathrm{kDa}$ & 11 & 5 \\
\hline Q9CDZ9_LACLA & $31 \mathrm{kDa}$ & 13 & 6 \\
\hline PRSA_LACLA & $34 \mathrm{kDa}$ & 13 & 6 \\
\hline NADE_LACLA & $30 \mathrm{kDa}$ & 44 & 21 \\
\hline QUEA_LACLA & $39 \mathrm{kDa}$ & 75 & 36 \\
\hline TYSY_LACLA & $33 \mathrm{kDa}$ & 15 & 7 \\
\hline Q9CEI5_LACLA & $40 \mathrm{kDa}$ & 15 & 7 \\
\hline Q9CIK0_LACLA & $15 \mathrm{kDa}$ & 15 & 7 \\
\hline RS5_LACLA & $18 \mathrm{kDa}$ & 157 & 76 \\
\hline MURD_LACLA & $49 \mathrm{kDa}$ & 19 & 9 \\
\hline Q9CEX8_LACLA & $35 \mathrm{kDa}$ & 79 & 39 \\
\hline TPIS_LACLA & $27 \mathrm{kDa}$ & 7 & 15 \\
\hline Q9CHF8_LACLA & $34 \mathrm{kDa}$ & 4 & 9 \\
\hline
\end{tabular}


Protein-tyrosine phosphatase

1,4-dihydroxy-2-naphthoyl-CoA synthase

Isoleucine--tRNA ligase

3-hydroxyacyl-[acyl-carrier-protein]

dehydratase FabZ

Transcription termination/antitermination protein NusA

Exodeoxyribonuclease A

Dihydrolipoamide acetyltransferase

component of PDH complex

Uncharacterized protein

DNA replication protein DnaD

Ribonuclease 3

Uncharacterized protein

Ferrous ion transport protein A

Uncharacterized protein

Mevalonate kinase

Uncharacterized protein

tRNA dimethylallyltransferase

DNA-directed RNA polymerase subunit omega

Uncharacterized protein

Putative gluconeogenesis factor

Uncharacterized protein

Bifunctional protein GlmU

Uncharacterized protein

Valine--tRNA ligase

Leucine--tRNA ligase

DNA polymerase III PolC-type

Non-heme chloride peroxidase

Endoribonuclease YbeY

Transcriptional regulator

Uncharacterized protein

Prophage pi3 protein 57, cI-like repressor

Pyruvate carboxylase

\begin{tabular}{|c|c|c|c|c|}
\hline Q9CEK1_LACLA & $28 \mathrm{kDa}$ & 4 & 9 & 0,47 \\
\hline Q9CHK2_LACLA & $31 \mathrm{kDa}$ & 18 & 39 & 0,47 \\
\hline SYI_LACLA & $107 \mathrm{kDa}$ & 3 & 7 & 0,47 \\
\hline FABZ2_LACLA & $16 \mathrm{kDa}$ & 3 & 7 & 0 , \\
\hline Q9CHG7_LACLA & $43 \mathrm{kDa}$ & 100 & 220 & 0 \\
\hline Q9CHE1_LACLA & $38 \mathrm{kDa}$ & 6 & 14 & 0 \\
\hline Q9CJD7_LACLA & $56 \mathrm{kDa}$ & 42 & 95 & 0,4 \\
\hline Q9CIG7_LACLA & $31 \mathrm{kDa}$ & 5 & 12 & 0, \\
\hline Q9CGM6_LACLA & $26 \mathrm{kDa}$ & 8 & 19 & 0,4 \\
\hline RNC_LACLA & $26 \mathrm{kDa}$ & 10 & 25 & 0, \\
\hline Q9CE54_LACLA & $32 \mathrm{kDa}$ & 7 & 18 & 0,4 \\
\hline Q9CJ18_LACLA & $17 \mathrm{kDa}$ & 8 & 21 & 0, \\
\hline Q9CHB7_LACLA & $26 \mathrm{kDa}$ & 4 & 11 & 0,3 \\
\hline Q9CIF6_LACLA & $36 \mathrm{kDa}$ & 2 & 6 & 0,3 \\
\hline Q9CEM6_LACLA & $31 \mathrm{kDa}$ & 2 & 6 & 0,3 \\
\hline MIAA_LACLA & $34 \mathrm{kDa}$ & 2 & 6 & 0,3 \\
\hline RPOZ_LACLA & $13 \mathrm{kDa}$ & 2 & 6 & \\
\hline Q9CIJ7_LACLA & $23 \mathrm{kDa}$ & 19 & 52 & 0,3 \\
\hline GNGF_LACLA & $36 \mathrm{kDa}$ & 3 & 9 & 0,3 \\
\hline Q9CJ34_LACLA & $41 \mathrm{kDa}$ & 4 & 12 & 0 \\
\hline GLMU_LACLA & $49 \mathrm{kDa}$ & 8 & 27 & 0,3 \\
\hline Q9CHZ8_LACLA & $28 \mathrm{kDa}$ & 2 & 9 & 0 \\
\hline SYV_LACLA & $101 \mathrm{kDa}$ & 3 & 13 & 0,2 \\
\hline SYL_LACLA & $94 \mathrm{kDa}$ & 3 & 1 & 0 \\
\hline DPO3_LACLA & $185 \mathrm{kDa}$ & 11 & 69 & 0 \\
\hline Q9CHB2_LACLA & $31 \mathrm{kDa}$ & & 6 & 0 , \\
\hline YBEY_LACLA & $19 \mathrm{kDa}$ & & 7 & 0 , \\
\hline Q9CIF1_LACLA & $28 \mathrm{kDa}$ & & 8 & 0, \\
\hline Q9CHI3_LACLA & $30 \mathrm{kDa}$ & & 8 & 0 \\
\hline Q9CFN7_LACLA & $20 \mathrm{kDa}$ & & 10 & 0 , \\
\hline Q9CHQ7_LACLA & $126 \mathrm{kDa}$ & 51 & 2531 & 0 \\
\hline
\end{tabular}


Biotin carboxyl carrier protein of acetyl-

CoA carboxylase
Q9CHF5_LACLA $\mid 16 \mathrm{kDa}$

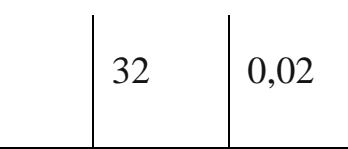

The dashed line indicates the cut off established for specific c-di-AMP interaction assay.

\subsection{Bacterial strains}

Table XV Bacterial strains

\begin{tabular}{|c|c|c|}
\hline Bacterial Strain & Genotype/Characteristics & Reference \\
\hline \multicolumn{3}{|l|}{ E. coli strains } \\
\hline E coli 2003 & $\triangle k d p A B C 5$ kupD1 $\triangle$ trkA & Hamann 1991 (174) \\
\hline E coli BL21 & $\begin{array}{l}\text { E. coli B } \mathrm{F}^{-} \text {ompT gal dcm lon } h s d S_{B}\left(r_{B}^{-} m_{B}^{-}\right) \\
{\left[m a l B^{+}\right]_{\mathrm{K}-12}\left(\lambda^{\mathrm{S}}\right)}\end{array}$ & $\begin{array}{l}\text { Studier and Moffatt } \\
1986(175)\end{array}$ \\
\hline E. coli 0300 & E. coli $2003+$ pIQ310 and pBP370 $-\mathrm{Cm}^{30}-\mathrm{Amp}^{100}$ & This work \\
\hline E. coli 0303 & E. coli $2003+$ pIQ310 and pBP373 $-\mathrm{Cm}^{30}-\mathrm{Amp}^{100}$ & This work \\
\hline E. coli 0380 & $\begin{array}{l}\text { E. coli } 2003+\text { pWH844 and pBP370 }-\mathrm{Cm}^{30}- \\
\text { Amp }^{100}\end{array}$ & This work \\
\hline E. coli 0383 & $\begin{array}{l}\text { E. coli } 2003+\text { pWH844 and pBP373 }-\mathrm{Cm}^{30}- \\
\text { Amp }^{100}\end{array}$ & This work \\
\hline E. coli 0390 & E. coli $2003+$ pIQ309 and pBP370 $-\mathrm{Cm}^{30}-\mathrm{Amp}^{100}$ & This work \\
\hline E. coli 0393 & E. coli $2003+$ pIQ309 and pBP373 $-\mathrm{Cm}^{30}-\mathrm{Amp}^{100}$ & This work \\
\hline E. coli DH5 $\alpha$ & $\begin{array}{l}\Delta \text { (lacZ)M15 gyrA96 recA1 relA1 endA1 thi-1 } \\
\text { hsdR17 }\end{array}$ & Hanahan, 1983 (176) \\
\hline E. coli EC101 & $\begin{array}{l}\text { E. coli JM101 derived. } \text { repA }^{+} \text {(from pWV01 } \\
\text { integrated in chromosome) } \mathrm{Km}^{40}\end{array}$ & Law et al. 1995 (177) \\
\hline E. coli GP01 & E. coli BL21 + pIQ402 & This work \\
\hline E. coli GP02 & E. coli BL21 + pIQ404 & This work \\
\hline E. coli LB08 & E. coli LB650 + pWH844 $-\mathrm{Km}^{50}-\mathrm{Cm}^{30}-\mathrm{Amp}^{100}$ & This work \\
\hline E. coli LB09 & E. coli LB650 + pIQ309 $-\mathrm{Km}^{50}-\mathrm{Cm}^{30}-\mathrm{Amp}^{100}$ & This work \\
\hline E. coli LB10 & E. coli LB650 + pIQ310 $-\mathrm{Km}^{50}-\mathrm{Cm}^{30}-\mathrm{Amp}^{100}$ & This work \\
\hline E. coli LB11 & E. coli LB650 + pBP372 $-\mathrm{Km}^{50}-\mathrm{Cm}^{30}-\mathrm{Amp}^{100}$ & This work \\
\hline E. coli LB12 & E. coli LB650 + pBP371 $-\mathrm{Km}^{50}-\mathrm{Cm}^{30}-\mathrm{Amp}^{100}$ & This work \\
\hline E. coli LB13 & E. coli LB650 + pIQ311 $-\mathrm{Km}^{50}-\mathrm{Cm}^{30}-\mathrm{Amp}^{100}$ & This work \\
\hline E. coli LB650 & $\Delta k d p A B C 5 \Delta \operatorname{trk} H \Delta \operatorname{trk} G-\mathrm{Km}^{50}-\mathrm{Cm}^{30}$ & Schlösser et al. 1995 \\
\hline E. coli YB01 & E. coli BL21 + pIQ401 & This work \\
\hline E. coli YB02 & E. coli BL21 + pIQ403 & This work \\
\hline
\end{tabular}

\section{E. faecalis strains}




\begin{tabular}{|c|c|c|}
\hline E. faecalis JH2-2 & Cit $^{+}$; Rif ${ }^{\mathrm{R}}$; Fus ${ }^{\mathrm{R}}$; plasmid free & Jacob and Hobbs, 1974 \\
\hline \multicolumn{3}{|l|}{ L. lactis strains } \\
\hline L. lactis htrA-clpP & $\begin{array}{l}\text { NZ9000 transconjugant carrying } c l p P \text { and } h t r A \\
\text { disruption, plasmid free }-\mathrm{Em}^{5}\end{array}$ & Cortes-Perez 2006 \\
\hline L. lactis IL1403 & Derived from IL594 strain. Plasmid free, $\operatorname{Trp}^{+}$ & Chopin et al., 1984 \\
\hline L. lactis LL00 & L. lactis IL1403 + pBV153 & This work \\
\hline L. lactis LL01 & L. lactis NZ9000 clpP-htrA + pNZ8048 & This work \\
\hline L. lactis LL02 & L. lactis NZ9000 clpP-htrA + pNZTS & This work \\
\hline L. lactis LL03 & L. lactis IL1403 + pIQ101 & This work \\
\hline L. lactis LL04 & L. lactis IL1403 + pIQ102 & This work \\
\hline L. lactis LL05 & L. lactis IL1403 + pIQ103 & This work \\
\hline L. lactis LL06 & L. lactis IL1403 + pIQ104 & This work \\
\hline L. lactis LL07 & L. lactis IL1403 $\triangle g d p P 1 s$ & This work \\
\hline L. lactis LL08 & L. lactis IL1403 $\triangle g d p P 1 b$ & This work \\
\hline L. lactis LL09 & L. lactis NZ9000 clpP-htrA + pIQ101 & This work \\
\hline L. lactis LL10 & L. lactis NZ9000 clpP-htrA + pIQ10TS & This work \\
\hline L. lactis NZ9000 & $\begin{array}{l}\text { L. lactis MG1363 containing nisKR genes integrated } \\
\text { into pepN locus, plasmid free }\end{array}$ & Kuipers 1998 \\
\hline L. lactis NZTS & L. lactis NZ9000+ pNZTS & This work \\
\hline
\end{tabular}

\subsection{Plasmids}

Table XVI Plasmids

\begin{tabular}{|c|c|c|}
\hline Plasmid & Description & Reference \\
\hline pBAD33 & arabinose inducible $-\mathrm{Cm}^{\mathrm{R}}$ & $\begin{array}{l}\text { Guzman et al. } 1995 \\
\text { (178) }\end{array}$ \\
\hline pBP371 & pWH844 ktrC/D L. monocytogenes & Stülke Lab \\
\hline pBP372 & pWH844 ktrA/B B. subtilis & Stülke Lab \\
\hline pBV153 & $\begin{array}{l}\text { PcitM promoter, } \mathrm{pH} \text { inducible } \mathrm{Amp}^{\mathrm{R}} \text { in } E \text {. coli, } \\
\mathrm{Cm}^{\mathrm{R}} \text { in } \mathrm{LAB}\end{array}$ & $\begin{array}{l}\text { Marelli and Magni } \\
2009\end{array}$ \\
\hline pBVGh & Thermosensitive vector for gene deletion $\mathrm{Em}^{\mathrm{R}}$ & $\begin{array}{l}\text { Blancato and Magni } \\
2010\end{array}$ \\
\hline pIQ101 & pBV153 + L. lactis cdaAll (Pcit promoter) & This work \\
\hline pIQ102 & pBV153 + L. lactis cdaA ${ }^{e f}$ (Pcit promoter) & This work \\
\hline pIQ103 & pBV153 + L. lactis gdpP $P^{l l}$ (Pcit promoter) & This work \\
\hline pIQ104 & pBV153 + L. lactis gdpP $P^{e f}$ (Pcit promoter) & This work \\
\hline
\end{tabular}


pIQ101 derived plasmid encoding cdaA gene

\begin{tabular}{|c|c|c|}
\hline pIQ10TS & $\begin{array}{l}\text { under PcitM promoter and trans-sialidase } \\
\text { fragment under Pnis promoter }\end{array}$ & This work \\
\hline pIQ309 & pWH844 no His-tag + kupA IL1403 & This work \\
\hline pIQ310 & pWH844 no His-tag + kupB IL1403 & This work \\
\hline pIQ311 & pWH844 + kup JH2-2 & This work \\
\hline pIQ370 & $\mathrm{pBAD} 33+c d a A^{\operatorname{lmo}}$ & This work \\
\hline pIQ373 & pBAD33 + cdaA D171N Lmo & This work \\
\hline pIQ401 & pWH844 + yheB L. lactis NZ9000 & This work \\
\hline pIQ402 & pWH844 + gdpP L. lactis NZ9000 & This work \\
\hline pIQ403 & pWH844 + yheB L. lactis IL1403 & This work \\
\hline pIQ404 & pWH844 + gdpP L. lactis IL1403 & This work \\
\hline pNZ8048 & Nisin inducible expression, $\mathrm{Cm}^{\mathrm{R}}$ & $\begin{array}{l}\text { De Ruyter et al. } \\
1996(179)\end{array}$ \\
\hline pNZTS & $\begin{array}{l}\text { pNZ8048 + codon optimized His } \text { Hx }_{6} \text {-tagged trans- } \\
\text { sialidase fragment }\end{array}$ & This work \\
\hline pUC57-TS & $\begin{array}{l}\text { pUC57 + codon optimized } \text { His }_{6 x} \text {-tagged trans- } \\
\text { sialidase fragment }\end{array}$ & This work \\
\hline pWH844 & IPTG inducible - $\mathrm{N}$ terminal His tag, $\mathrm{Amp}^{\mathrm{R}}$ & Stülke Lab \\
\hline pWHdnaH & pWH844 no His-tag + RBS + dnaH & $\begin{array}{l}\text { Grubek, Z. } 2016 \\
(180)\end{array}$ \\
\hline pWHezrA & pWH844 no His-tag + RBS + ezrA & Grubek, Z. 2016 \\
\hline pWHftsE & pWH844 no His-tag + RBS + ftsE & Grubek, Z. 2016 \\
\hline pWHglnP & pWH844 no His-tag + RBS $+g \ln P$ & Grubek, Z. 2016 \\
\hline pWHkupA & pWH844 no His-tag + RBS + kupA & Grubek, Z. 2016 \\
\hline pWHkupB & pWH844 no His-tag + RBS + kupB & Grubek, Z. 2016 \\
\hline pWHlysP & pWH844 no His-tag + RBS + lysP & Grubek, Z. 2016 \\
\hline pWHlysQ & pWH844 no His-tag + RBS + lysQ & Grubek, Z. 2016 \\
\hline pWHmvaA & pWH844 no His-tag + RBS + mvaA & Grubek, Z. 2016 \\
\hline pWHnah & pWH844 no His-tag + RBS + nah & Grubek, Z. 2016 \\
\hline pWHoppA & pWH844 no His-tag + RBS + oppA & Grubek, Z. 2016 \\
\hline pWHpdp & pWH844 no His-tag + RBS $+p d p$ & Grubek, Z. 2016 \\
\hline pWHprfB & pWH844 no His-tag + RBS + prfB & Grubek, Z. 2016 \\
\hline pWHracD & pWH844 no His-tag + RBS + racD & Grubek, Z. 2016 \\
\hline pWHrecG & pWH844 no His-tag + RBS + recG & Grubek, Z. 2016 \\
\hline pWHrecJ & pWH844 no His-tag + RBS + recJ & Grubek, Z. 2016 \\
\hline
\end{tabular}




$\begin{array}{lll}\text { pWHrecN } & \text { pWH844 no His-tag + RBS + recN } & \text { Grubek, Z. } 2016 \\ \text { pWHrelA } & \text { pWH844 no His-tag + RBS + relA } & \text { Grubek, Z. } 2016 \\ \text { pWHribH } & \text { pWH844 no His-tag + RBS + ribH } & \text { Grubek, Z. } 2016 \\ \text { pWHrpsF } & \text { pWH844 no His-tag + RBS + rpsF } & \text { Grubek, Z. } 2016 \\ \text { pWHyciH } & \text { pWH844 no His-tag + RBS }+y c i H & \text { Grubek, Z. } 2016 \\ \text { pWHyeaD } & \text { pWH844 no His-tag + RBS + yeaD } & \text { Grubek, Z. } 2016 \\ \text { pWHyhfC } & \text { pWH844 no His-tag + RBS }+y h f C & \text { Grubek, Z. } 2016 \\ \text { pWHyjdJ } & \text { pWH844 no His-tag + RBS }+y j d J & \text { Grubek, Z. } 2016 \\ \text { pWHyncB } & \text { pWH844 no His-tag + RBS }+y n c B & \text { Grubek, Z. } 2016 \\ \text { pWHyqaB } & \text { pWH844 no His-tag + RBS }+y q a B & \text { Grubek, Z. } 2016 \\ \text { pWHyqaD } & \text { pWH844 no His-tag + RBS }+y q a D & \text { Grubek, Z. } 2016 \\ \text { pWHyrbD } & \text { pWH844 no His-tag + RBS }+y r b D & \text { Grubek, Z. } 2016 \\ \text { pWHyrgI } & \text { pWH844 no His-tag + RBS }+y r g I & \text { Grubek, Z. } 2016 \\ \text { pWHyrjB } & \text { pWH844 no His-tag + RBS }+y r j B & \text { Grubek, Z. } 2016 \\ \text { pWHytcE } & \text { pWH844 no His-tag + RBS }+y t c E & \text { Grubek, Z. } 2016 \\ \text { pWHytjA } & \text { pWH844 no His-tag + RBS }+y t j A & \text { Grubek, Z. } 2016 \\ \text { pWHyueF } & \text { pWH844 no His-tag + RBS }+y u e F & \text { Grubek, Z. } 2016 \\ \text { pWHywfH } & \text { pWH844 no His-tag + RBS }+y w f H & \text { Grubek, Z. } 2016\end{array}$

\subsection{Oligonucleotides}

Homologous sequences hybridizing with template DNA are in italics, restriction sites are underlined. Fwd: forward. Rv: reverse.

Table XVII Oligonucleotides

\begin{tabular}{|c|c|c|}
\hline Name & Sequence (5’ -> 3') & Purpose \\
\hline IQ365 & ACAAGTACATATGATGCTTGTTGTGGAAC & (Fwd) cdaA - E. faecalis JH2-2 \\
\hline IQ366 & GCACTAGTAAGCTTTCATTTTGCGTTTAACC & (Rv) cdaA - E. faecalis JH2-2 \\
\hline IQ367 & AACTAGACATATGATGCAAAAGAAGAGAATTC & (Fwd) gdpP - E. faecalis JH2-2 \\
\hline IQ368 & CGACTAGTAAGCTTTCACTCCTGTTCATAC & (Rv) gdpP - E. faecalis JH2-2 \\
\hline IQ369 & ACGTAACCATATGTTGACCGACTTCAATC & (Fwd) cdaA - L. lactis IL1403 \\
\hline IQ370 & GCTCTAGAAAGCTTTTATTTGCCATTTTTC & (Rv) cdaA - L. lactis IL1403 \\
\hline JN371 & AAACCATGGAGAACGTGAAGCAAAAGCACT & $\begin{array}{l}\text { (Fwd) Upstream region of } c d a A- \\
\text { L. lactis IL1403 }\end{array}$ \\
\hline JN372 & TTTAAGCTTCGAACAAGCGTCATTAATTTTGT & $\begin{array}{l}\text { (Rv) Upstream region of } c d a A-L \text {. } \\
\text { lactis IL1403 }\end{array}$ \\
\hline
\end{tabular}


JN373 AAAAAGCTTTTGCTCACAATGGAGAATTTTTCG

JN374 TTTCCATGGCAGAAAGATGAACCGTCGCA

JN375 AAACCATGGCCGTTTGGGCAATTGAAGACA

JN376 TTTAAGCTTATTAAAACGGATGACCCCAATTG

JN377 AAAAAGCTTATTATGGAGCAAATGGGTGGG

JN378 TTTCCATGGGCTTTTCTTTTTCCTTAGCTTTGG

JN379 AAACATATGGGAGTAATATTATGAATTTTGTCC $G$

JN380 TTTACTAGTAAGAATACAACTTTCATTTTTCGTT

JN384 TTTTCTAGATTATTTGCCATTTTTCTTTCCTCCT

JN385 TATTTGGGAATAAAGCAGTTCACTTAAAA

JN386 GAAAGTAACTGTTCCTATACGTGC

JN387 CAAAAGTCCTGAAATCAAACTGCT

JN388 AAAAAAGATAAAAAAGCTCTCCCAATAAG

JN389 TGGTTGACATTTTTCTTTGATTGTTATC

JN400 AAACCATGGCCGTTTGGG

JN401 TTTCCATGGGCTTTTCTTTTTCC

JN402 AAACCATGGAGAACGTGAAG

JN403 TTTCCATGGCAGAAAGATGAA

JN404 AAAATGCGAGCGATGACCAA
(Fwd) Downstream region of cdaA - L. lactis IL1403

(Rv) Downstream region of $c d a A$ - L. lactis IL1403

(Fwd) Upstream region of $g d p P$ L. lactis IL1403

(Rv) Upstream region of $g d p P$ L. lactis IL1403

(Fwd) Downstream region of gdpP - L. lactis IL1403

(Rv) Downstream region of $g d p P$ - L. lactis IL1403

(Fwd) gdpP - L. lactis IL1403

(Rv) gdpP - L. lactis IL1403

(Rv) cdaA - L. lactis IL1403 (pNZ8048)

(Fwd) $c d a A$ operon sequencing

(Fwd) $c d a A$ operon sequencing

(Rv) cdaA operon sequencing

(Rv) $c d a A$ operon sequencing

(Rv) gdpP IL1403 sequencing

(Fwd) ligation 58 amplification with JN401

(Rv) ligation 58 amplification with JN400

(Fwd) ligation 14 amplification with JN403

(Rv) ligation 14 amplification with JN402

(Fwd) $g d p P$ internal fragment $-L$. lactis IL1403 
$\begin{array}{ll}\text { JN405 } & \text { TTAATGGCTGTTCGACCGCT } \\ \text { JN406 } & \text { GGTTCTATGAAATTTAAAGCAGTGATTT } \\ \text { JN407 } & \text { TTAGGCCTCGCTAATTTTGACTT } \\ \text { JN408 } & \text { AAA } \underline{ }\end{array}$

JN409 TTTGAATTCAAGCGTCATTAATTTTGTTCCT

JN410 AAAGAATTCTAGCACATAATGGCGAGTTT

JN411 TTTGAGCTCTAAATGAACTGTTGCACTGC

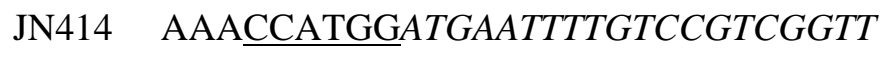

JN416 CGATAACGCGAGCATAATAAAC

JN417 GCCTTGGTTTTCTAATTTTGGTTC

JN418 GTTTAAACGCTTTGGGACGT

JN419 GTATCGACGTTGACTTGCTT

JN500 TTTGGATCCATGACCTATCGGATGGTTGA

JN501 AAAAAGCTTTTATCTGATAATTTCTAGAATTTCT TTTTGA

JN502 AAAGGATCCATGGAATTATCAGAAATTAGAAAT TTACTT

JN503 TTTAAGCTTCTACAAGTTCCAACGGAGATA

JN504 TTTGGATCCATGGCATATCAAGCATTATATAGAA AAT

JN505 AAAGTCGACTTAATCATTAATTTCAACTACTTTT TCACC
(Rv) gdpP internal fragment - $L$. lactis IL1403

(Fwd) gene upstream gdpP

IL1403 Deletion check

(Rv) gene downstream $g d p P$

IL1403 Deletion check

(Fwd) Upstream region of $c d a A L$. lactis NZ9000 / deletion with pBVGh

(Rv) Upstream region of $c d a A \mathrm{~L}$. lactis NZ9000 / deletion with pBVGh

(Fwd) Downstream region of cdaA L. lactis NZ9000 / deletion with pBVGh

(Rv) Downstream region of $c d a A$ L. lactis NZ9000 / deletion with pBVGh

(Fwd) cdaA - L. lactis IL1403 (pNZ8048)

(Fwd) pNZ8048 checking

(Rv) pNZ8048 checking

(Fwd) recJ check

(Fwd) $g \ln P$ check

(Rv) $p d p$ / construction in pWH844 / DRaCALA

(Fwd) $p d p /$ construction in pWH844 / DRaCALA

(Fwd) prfB / construction in pWH844 / DRaCALA

(Rv) $p r f B /$ construction in pWH844 / DRaCALA

(Rv) dnaH / construction in pWH844 / DRaCALA

(Fwd) dnaH / construction in pWH844 / DRaCALA 
JN506 TTTGGATCCATGAAATTAACAGATTCAGTGCAAT TTTT

JN507 AAAAAGCTTTCAATCAAAGCCCCCGTCA

JN508 AAAGGATCCATGATAAAAGCAAAATATGATTGG $A A A$

JN509 TTTAAGCTTTTATTTTTCTAATAAATTTTGATAAA TTTCTTT

JN510 AAAGGATCCATGTTACAAGAGATTTCAATCAAA AATTT

JN511 TTTGTCGACTTATTTGCTCAATAAACGTTTAGCC

JN512 AAAGGATCCATGGCTGATAAATATAATGTTTTC GA

JN513 TTTAAGCTTTCAGCGATACAAAAGCTCCA

JN514 TTTGGATCCATGACTAAATACGAAATTCTTTATA TTATTCG

JN515 AAAGTCGACTTAAGCTTCAACTTTAACGATCATA TG

JN516 TTTGGATCCATGCCAAAGCAACTTAAAATAAAAG

JN517 AAAGTCGACTTATTCATCAGGTGTCATAATCATT $G$

JN518 AAAGGATCCATGAGTATTATAAAATTAAGCAAC GTTTC

JN519 TTTAAGCTTCTAATCATCGTAGCCGTAAACT

JN520 TTTGGATCCATGAATAAAAAATCGAGTGCTCTTT

JN521 AAAAAGCTTCTACTCCATTATAGTAAGTATCCA $G C$

JN522 AAAGGATCCATGAAGAAATTATTTTTCGCTCTG $G$

JN523 TTTAAGCTTTTATTTCATTCTTTTTTCTACGCGA

JN524 TTTGGATCCATGGAAAACTTCTTTACAATCTTGG
(Rv) recG / construction in pWH844 / DRaCALA

(Fwd) rec $G$ / construction in pWH844 / DRaCALA

(Fwd) recJ / construction in pWH844 / DRaCALA

(Rv) recJ / construction in pWH844 / DRaCALA

(Fwd) recN / construction in pWH844 / DRaCALA

(Rv) $\mathrm{recN} /$ construction in pWH844 / DRaCALA

(Fwd) yeaD / construction in pWH844 / DRaCALA

(Rv) yeaD / construction in pWH844 / DRaCALA

(Rv) $r p s F /$ construction in pWH844 / DRaCALA

(Fwd) $r p s F /$ construction in pWH844 / DRaCALA

(Rv) $y c i H$ / construction in pWH844 / DRaCALA

(Fwd) $y c i H /$ construction in pWH844 / DRaCALA

(Fwd) ftsE / construction in pWH844 / DRaCALA

(Rv) ftsE / construction in pWH844 / DRaCALA

(Rv) $y w f H /$ construction in pWH844 / DRaCALA

(Fwd) $y w f H /$ construction in pWH844 / DRaCALA

(Fwd) $g \ln P$ / construction in pWH844 / DRaCALA

(Rv) $g \ln P$ / construction in pWH844 / DRaCALA

(Rv) $\mathrm{racD}$ / construction in pWH844 / DRaCALA 
JN525 AAAAAGCTTTTAATAGTCATCTAATAAATCTTTT TTTCCC

JN526 TTTGGATCCATGAGAAAAAAATTTTATCAAATGT CGC

JN527 AAAGTCGACTTAGTTTCTGAGACTATTTAATAAA CGG

JN528 AAAGGATCCATGAAAGTTTCAATATTCTCCACTT $G$

JN529 TTTAAGCTTCTATGCTTTGATAAATGCTGGGT

JN530 TTTGGATCCATGCAAGCAAAATTAGTTAATAAAG TAG

JN531 AAAAAGCTTTTAATGTCTTTGCTCAATATTTTGC $T$

JN532 TTTGGATCCATGCTTCATCTCATTAAAGAAAGC

JN533 AAAAAGCTTTTACTTTTCAAGGCTGACCTC

JN534 AAAGGATCCATGCCTAAAGAACCAGATTTAACC

JN535 TTTGTCGACTTAAGCATTTGTCCGTTTTACAGA

JN536 AAAGGATCCATGACAATTATTGAAGGAAATTTA AGAAC

JN537 TTTAAGCTTTTAGCCAATCTTTCTCATTAAATCA A

JN538 AAAGGATCCATGACTGAAGTTTATTTTATTCGAC AT

JN539 TTTAAGCTTTTATTGCTCATAATCAATTTCAATG ACT

JN540 TTTGGATCCATGAGTGAATTAGAGATTCGTAGA TT

JN541 AAAGTCGACCTATTGCTCTTCTAAATTAATCCAG $T A A$

JN542 AAAGGATCCATGGATAACCAAAATCAAGAAATA GG

JN543 TTTAAGCTTTTATGCGTTTGGATAGTGAATTAAA A
(Fwd) racD / construction in pWH844 / DRaCALA

(Rv) mvaA / construction in pWH844 / DRaCALA

(Fwd) $m v a A$ / construction in pWH844 / DRaCALA

(Fwd) $y r j B$ / construction in pWH844 / DRaCALA

(Rv) $y r j B /$ construction in pWH844 / DRaCALA

(Rv) $y t j A$ / construction in pWH844 / DRaCALA

(Fwd) $y t j A /$ construction in pWH844 / DRaCALA

(Rv) yueF / construction in pWH844 / DRaCALA

(Fwd) yueF / construction in pWH844 / DRaCALA

(Fwd) relA / construction in pWH844 / DRaCALA

(Rv) relA / construction in pWH844 / DRaCALA

(Fwd) ribH / construction in pWH844 / DRaCALA

(Rv) $\mathrm{ribH} /$ construction in pWH844 / DRaCALA

(Fwd) yrgI / construction in pWH844 / DRaCALA

(Rv) yrgI / construction in pWH844 / DRaCALA

(Rv) yqaB / construction in pWH844 / DRaCALA

(Fwd) yqaB / construction in pWH844 / DRaCALA

(Fwd) yhfC / construction in pWH844 / DRaCALA

(Rv) yhfC / construction in pWH844 / DRaCALA 
JN544 TTTGGATCCATGTCAAGTACTGTCATTATCCTC

JN545 AAAAAGCTTTTATAAGTAATCAGGAGTAGGTTT ATTTTT

JN546 AAAGGATCCATGGCATTTATCACCGAAAAAC

JN547 TTTAAGCTTTTAAATTTCTGATTTTGTAAATACAC CG

JN548 TTTGGATCCATGGCTTTAACGATCGAACG

JN549 AAAAAGCTTTTAAAATCCCAAGTTTATTCCAGG C

JN550 TTTGGATCCATGAAATTAAAAGATATAGAGAAAT CAACAAA

JN551 AAAAAGCTTTTATTTTTCATTTAAAACCTGACGA $A G$

JN552 AAAGGATCCATGTTTGGAAAGTTTAATCGCTC

JN553 TTTAAGCTTTCATTTATTTTTTCTATTATTTCCTT CTAAGG

JN554 TTTGGATCCATGAATGATATTTTACAACTCACAA TTG

JN555 AAAGTCGACTTAATTATCATATTTTTTCAAGACC ATTTTAATT

JN556 AAAGTCGACTTGAGTCTGAGTCAAACAAGTC

JN557 TTTAAGCTTTTAATCTTTATGACGACTTAAATCA ACC

JN558 AAAGGATCCATGGAAAATCAAAATCAGGTCAAG

JN559 TTTAAGCTTTTATTTTTCCCGACTCAAATCTACT

JN560 AAAGGATCCATGGGTTATGAATCTAATCGCTC

JN561 TTTGTCGACTTATGATTCTCCTTGATTTTCTTCT G

JN562 AAAGGATCCGTGGGTCAAGTACACTTACATAA
(Rv) ezrA / construction in pWH844 / DRaCALA

(Fwd) ezrA / construction in pWH844 / DRaCALA

(Fwd) ytcE / construction in pWH844 / DRaCALA

(Rv) ytcE / construction in pWH844 / DRaCALA

(Rv) yqaD / construction in pWH844 / DRaCALA

(Fwd) yqaD / construction in pWH844 / DRaCALA

(Rv) yrbD / construction in pWH844 / DRaCALA

(Fwd) yrbD / construction in pWH844 / DRaCALA

(Fwd) yjdJ / construction in pWH844 / DRaCALA

(Rv) yjdJ / construction in pWH844 / DRaCALA

(Rv) nah / construction in pWH844 / DRaCALA

(Fwd) nah / construction in pWH844 / DRaCALA

(Fwd) lysP / construction in pWH844 / DRaCALA

(Rv) lysP / construction in pWH844 / DRaCALA

(Fwd) lysQ / construction in pWH844 / DRaCALA

(Rv) lys $Q$ / construction in pWH844 / DRaCALA

(Fwd) kupA / construction in pWH844 / DRaCALA

(Rv) kupA / construction in pWH844 / DRaCALA

(Fwd) kupB / construction in pWH844 / DRaCALA 
JN563 TTTGTCGACTTAATGAGTTGCTGAAGCTTCT

JN564 AAAGGATCCATGAACTTATTAAAAACAAACTGG GA

JN565 TTTAAGCTTTTATTTTTCATAATATTTATCTAAAA TCTCCATTTT

JN566 TTTGGATCCATGAAAAAATTAAAAGTAACTTTAT TGGCA

JN567 AAAAAGCTTCTATTTGGTTGCCATCTTATCAGA

JN568 AAAGGATCCATGATAAAAGCAAAATATGATTGG $A A A G$

JN569 TTTAAGCTTACGAAATTATTTTTCTAATAAATTTT GATAAATTTC

JN581 AGGTTATATTCATGACCTTTGGAAA

JN586 CGCTTGTTCGTGGTGTGATT

JN587 CGTAAGCTGAAATATGCCCATCA

IQ668 AAAGGATCCAAGGTACCAATGAACGAAATTTTA GAAAAAATAAAAGC

IQ669 TTTGGATCCCTGCAGTTATTTTAAATTATCTTGT AATTCTTGCC

IQ670 TTTGGATCCTTGGTACCAATGAACGAAATTTTAG AAAAAATTAAAGCC

IQ671 AAAGGATCCCTGCAGTTATTTCAAGTTATCTTGT AACTCTTG

IQ682 AAAGAATTCATTAAAGAGGAGAAAATTAGAATG GGTTATGAATCTAATCG

IQ683 AAAGAATTCATTAAAGAGGAGAAAACAAAAGTG GGTCAAGTACAC

IQ684 AAAAGATCTAGGTTTTTATATTACAGCTCCAGG A

IQ685 TTTAGATCTCTGGTATCTTTATAGTCCTGTCG

IQ687 AAAGGATCCGTGTTACACAAAGCAGAGGGG
(Rv) kupB / construction in pWH844 / DRaCALA

(Fwd) yncB / construction in pWH844 / DRaCALA

(Rv) $y n c B$ / construction in pWH844 / DRaCALA

(Rv) oppA / construction in pWH844 / DRaCALA

(Fwd) oppA / construction in pWH844 / DRaCALA

(Fwd) recJ / construction in pWH844 / DRaCALA

(Rv) recJ / construction in pWH844 / DRaCALA

(Fwd) relA check

(Fwd) internal fragment $c d a A-L$. lactis IL1403

$(R v)$ internal fragment $c d a A-L$. lactis IL1403

(Fwd) yheB - L. lactis IL1403 (pWH844)

(Rv) yheB - L. lactis IL1403 (pWH844)

(Rv) yheB - L. lactis NZ9000 (pWH844)

(Fwd) yheB - L. lactis NZ9000 (pWH844)

(Fwd) RBS+kupA L. lactis IL1403 (pWH844)

(Fwd) RBS+kupB L. lactis IL1403 (pWH844)

(Fwd) PcitM+cdaA from pIQ101

(Rv) PcitM $+c d a A$ from pIQ101

(Fwd) kup - E. faecalis JH2-2 


\begin{tabular}{|c|c|c|}
\hline IQ688 & $\begin{array}{l}\text { TTTGTCGACATTTCTTCTATTTATGAACGATTCTT } \\
\text { TC }\end{array}$ & (Rv) kup - E. faecalis JH2-2 \\
\hline IQ696 & $\begin{array}{l}\text { AAACTGCAGGTTGAAGAAGGTTTTTATATTACA } \\
\text { GC }\end{array}$ & (Fwd) hisTScf \\
\hline IQ697 & TTTGTCGACGGTGGACAAATTTACATTAGTCTC & (Rv) hisTScf \\
\hline IQ706 & ACAGGTACTGGCGCTAAAAAC & $\begin{array}{l}\text { (Fwd) External check (E. faecalis } \\
\text { JH2-2 gdpP deletion) }\end{array}$ \\
\hline IQ707 & GCTACGACATGAACCTTAATTTTC & $\begin{array}{l}\text { (Rv) External check (E. faecalis } \\
\text { JH2-2 gdpP deletion) }\end{array}$ \\
\hline IQ708 & CGGCTCAAACAAACCTAGATAC & $\begin{array}{l}\text { (Fwd) Internal check (E. faecalis } \\
\text { JH2-2 gdpP deletion) }\end{array}$ \\
\hline IQ709 & CTTTTGACATCGGGAATGATTTC & $\begin{array}{l}\text { (Rv) Internal check (E. faecalis } \\
\mathrm{JH} 2-2 \text { gdpP deletion) }\end{array}$ \\
\hline
\end{tabular}

\subsection{Materials}

\subsubsection{Buffers used in this work}

Table XVIII Buffers routinely used

\begin{tabular}{l|l|l}
\hline Solution & Composition & Concentration \\
\hline \multirow{3}{*}{ TAE } & Tris-HCl pH 8.0 & $40 \mathrm{mM}$ \\
& EDTA & $10 \mathrm{mM}$ \\
& Acetic acid & $0.057 \%$ \\
\hline \multirow{2}{*}{ TE } & Tris-HCl pH 8.0 & $10 \mathrm{mM}$ \\
& EDTA & $1 \mathrm{mM}$ \\
\hline \multirow{2}{*}{ Wash (W) } & Tris-HCl pH 7.5 & $100 \mathrm{mM}$ \\
& NaCl & $150 \mathrm{mM}$ \\
\hline \multirow{3}{*}{ Lysis buffer } & Tris-HCl & $30 \mathrm{mM}$ \\
& Urea & $8 \mathrm{M}$ \\
& Adjust to pH 8.0 & \\
\hline \multirow{3}{*}{ Sample Buffer } & Tris-HCl pH 7.0 & $50 \mathrm{mM}$ \\
& Dithiothreitol (DTT) & $0.5 \mathrm{mM}$ \\
& $\beta-$-mercaptoethanol & $4 \%(\mathrm{v} / \mathrm{v})$ \\
& SDS & $2 \%(\mathrm{p} / \mathrm{v})$ \\
& Bromophenol blue & $0,005 \%(\mathrm{p} / \mathrm{v})$ \\
& Glycerol & $15 \%(\mathrm{v} / \mathrm{v})$ \\
\hline \multirow{3}{*}{ Running buffer } & Tris Base pH 8.3 & $25 \mathrm{mM}$ \\
& Glycine (pH 8,3) & $192 \mathrm{mM}$ \\
& EDTA & $1 \mathrm{mM}$
\end{tabular}




\begin{tabular}{|c|c|c|}
\hline & SDS & $0,1 \%(\mathrm{p} / \mathrm{v})$ \\
\hline \multirow{3}{*}{ Buffer C } & Tris-HCl pH 7.4 & $50 \mathrm{mM}$ \\
\hline & $\mathrm{NaCl}$ & $500 \mathrm{mM}$ \\
\hline & Glycerol & $5 \%$ \\
\hline \multirow{5}{*}{ PBS } & $\mathrm{NaCl}$ & $8 \mathrm{~g} / \mathrm{l}$ \\
\hline & $\mathrm{KCl}$ & $0.2 \mathrm{~g} / \mathrm{l}$ \\
\hline & $\mathrm{Na}_{2} \mathrm{HPO}_{4}$ & $1.44 \mathrm{~g} / \mathrm{l}$ \\
\hline & $\mathrm{KH}_{2} \mathrm{PO}_{4}$ & $0.24 \mathrm{~g} / \mathrm{l}$ \\
\hline & Adjust $\mathrm{pH}$ to 7.3 & \\
\hline \multirow{5}{*}{ CP1 buffer } & $\mathrm{MgCl}_{2}$ & $1 \mathrm{mM}$ \\
\hline & Tris-HCl pH 8.0 & $5 \mathrm{mM}$ \\
\hline & $\mathrm{NaCl}$ & $230 \mathrm{mM}$ \\
\hline & DTT & $0.5 \mathrm{mM}$ \\
\hline & Protease inhibitor tablet & 1 unit \\
\hline \multirow{4}{*}{ CP2 buffer } & CP1 buffer & $1 \mathrm{X}$ \\
\hline & glycerol & $10 \%$ \\
\hline & BSA & $50 \mu \mathrm{g} / \mathrm{ml}$ \\
\hline & EDTA & $0.004 \%$ \\
\hline \multirow{4}{*}{ Buffer E } & Tris-HCl pH 8.0 & $100 \mathrm{mM}$ \\
\hline & $\mathrm{NaCl}$ & $1 \mathrm{mM}$ \\
\hline & EDTA & $1 \mathrm{mM}$ \\
\hline & D-desthiobiotin & $5 \mathrm{mM}$ \\
\hline
\end{tabular}

\subsubsection{Chemicals}

$\begin{array}{ll}\text { Acetic acid } & \text { ChemSolute/Merck } \\ \text { Acryl:Bisacrylamide } & \text { Roth } \\ \text { L-Arabinose } & \text { Sigma-Aldrich } \\ \text { c-di-AMP } & \text { Biolog } \\ \text { Agar } & \text { Roth } \\ \text { Agarose } & \text { PEQLAB/Genbiotech } \\ \text { AgNO3 } & \text { Roth } \\ \text { Ammonium peroxydisulfate } & \text { Roth } \\ \text { Ampicillin } & \text { Roth } \\ \text { BCIP } & \text { Sigma-Aldrich } \\ \text { Biotinylated c-di-AMP } & \text { Biolog } \\ \text { Bromophenol blue } & \text { Riedel-de Haën } \\ \text { BSA } & \text { AppliChem } \\ \text { CaCl2 2H2O } & \text { Roth }\end{array}$


Casamino acids (CAA)

CDP-Star

Chloramphenicol

Coomassie Brilliant Blue, G-250

\section{DAPI}

$D$-desthiobiotin

DMSO

dNTPs

Ethanol

Formaldehyde

$D$-(+)-Glucose

$L$-glutamic acid

Glycerol

Glycine

c-di-NMP

$\mathrm{HCl}$

HDGreen Plus DNA stain

Imidazole

Isopropyl $\beta$-D-1-thiogalactopyranoside

Kanamycin

$\mathrm{KCl}$

$\mathrm{KH}_{2} \mathrm{PO} 4$

$\mathrm{K}_{2} \mathrm{HPO}_{4} \cdot 3 \mathrm{H}_{2} \mathrm{O}$

$\mathrm{KOH}$

$\lambda$-DNA

$\beta$-mercaptoethanol

Methanol

$\mathrm{MgCl}_{2} \cdot 3 \mathrm{H}_{2} \mathrm{O}$

$\mathrm{MgSO}_{4} \cdot 7 \mathrm{H}_{2} \mathrm{O}$

$\mathrm{MnCl}_{2} \cdot 4 \mathrm{H}_{2} \mathrm{O}$

$\mathrm{NaCl}$

$\mathrm{Na}_{2} \mathrm{CO}_{3}$

$\mathrm{Na}_{2}$-EDTA · 2H2O

$\left(\mathrm{NH}_{4}\right)_{2} \mathrm{SO}_{4}$

$\mathrm{NaOH}$

$\mathrm{Na}_{2} \mathrm{~S}_{2} \mathrm{O}_{3} \cdot 5 \mathrm{H}_{2} \mathrm{O}$
Roth

Roche

Serva/Sigma-Aldrich

Roth

AppliChem

IBA

Roth

Thermo Scientific

VWR Prolabo chemicals/Merck

Roth

AppliChem

Roth

Roth/Merck

AppliChem/Sigma-Aldrich

Sigma-Aldrich

Biolog

Intas

Sigma-Aldrich

PEQLAB/Sigma-Aldrich

AppliChem

Roth/Merck

Roth/Merck

Roth/Merk

Roth/Merck

Thermo Scientific

Roth

VWR Chemicals/Merck

Roth/Sigma-Aldrich

Roth/ Sigma-Aldrich

Roth/ Sigma-Aldrich

Roth/ Sigma-Aldrich

Roth/ Sigma-Aldrich

Roth/ Sigma-Aldrich

Fluka

Roth/ Sigma-Aldrich

Merck 
$\mathrm{Ni}^{2+}$-NTA Sepharose

NBT

PageRulerTM Plus Protein Ladder

Protease Inhibitor Tablet

Sodium dodecyl sulfate (SDS)

Strep-Tactin Sepharose

$D$-(+)-Sucrose

Tris

Tryptone

L-Tryptophan

Tween-20

Yeast extract

X-Gal

Xylene cyanol
Sigma-Aldrich

IBA ICN

Thermo Scientific

Sigma

Roth

IBA

AppliChem/Sigma-Aldrich

Roth/Sigma-Aldrich

Roth/Oxoid

AppliChem

Sigma-Aldrich

Oxoid

Sigma-Aldrich

Merck

\subsubsection{Auxiliary material}

Centrifuge cups

Cuvettes (microlitre, plastic)

Eppendorf tubes

Falcon tubes

Glass beads $0.1 \mathrm{~mm}$

Glass pipettes

Micropipettes

Petri dishes

Pipette tips

Poly-prep chromatography columns

PVDF membrane

Nitrocellulose membrane

Vivaspin ${ }^{\circledR}$ turbo 15
Beckmann

Greiner

Greiner

Starstedt/

Roth

Brad

Eppendorf

Greiner

Greiner

BioRad

BioRad

GE Healthcare

Sartorius

\subsubsection{Instrumentation}

ÄKTAprime plus

Autoclave LTA 2x3x4
GE Healthcare

Zirbus Technology 
AxioCam MRm

AxioImager M2 Microscope

Axioskop 40 Microscope

Gene Pulser Xcell and PC Module

Centrifuge Heraeus FRESCO 21

Centrifuge Heraeus Megafuge 16R

Centrifuge Sorvall RC 6+

Centrifuge Sorvall WX Ultraseries

Chrom. Data System LC-NetII/ADC

ECPlan-NEOFLUAR 100X/1.3

French pressure cell press

Horizontal shaker 3006

Microplate reader

Ice machine MF 36

Incubator Heraeus

Incubator shaker

Labcycler

Magnetic stirrer KMO 2 basic

Mini PROTEAN® System

Molecular Imager ${ }^{\circledR}$ Gel Doc ${ }^{\mathrm{TM}} \mathrm{XR}+$

NanoDrop ND-1000

pH-meter Calimatic 766

Plan Neofluar 100x/1.30

Plan Neofluar 40x/0.75

Power Pac Basic ${ }^{\mathrm{TM}}$

Press machine G.

Scale CP22025

Ultrospec 2100 pro spectroph.

UV Detector UV-2075Plus

Sterile bench HERA safe KS12

HiLoad 16/600 Superdex 200 pg

ThermoStat Plus

Tyssue Lyser II

Vortex Schütt
Zeiss

Zeiss

Zeiss

Bio-Rad

Thermo Scientific

Thermo Scientific

Thermo Scientific

Thermo Scientific

Jasco

Zeiss

Thermo Scientific

GFL

BioTek

Scotsman

Thermo Scientific

Innova ${ }^{\circledR} 44$ series Eppendorf

SensoQuest

IKA-Werke

BioRad

BioRad

PeqLab Biotechnologie

Knick

Zeiss

Zeiss

BioRad

Heinemann

Sartorius

Amersham Biosciences

Jasco

Thermo Scientific

GE Healthcare

Eppendorf

QIAGEN

Labortechnik 
Water desalination plant

\subsubsection{Commercial systems}

DNeasy® Blood \& Tissue Kit

NucleoSpin ${ }^{\circledR}$ Plasmid

peqGOLD Bacterial DNA kit

QIAquick ${ }^{\circledR}$ PCR purification Kit

Roti®-Quant

\subsubsection{Enzymes and antibodies}

Alkaline phosphatase (AP)

DNase I

Phusion DNA polymerase

RNase A

Restriction enzymes

T4-DNA ligase

Anti-Rabbit IgG
Millipore

QIAGEN

Macherey-Nagel

PEQLAB

QIAGEN

Roth

Thermo Scientific

Roche

Thermo Scientific

Roche

Thermo Scientific

Thermo Scientific

Promega

\subsection{Informatic tools}

\subsubsection{Websites}

Tabla XIX Websites

\begin{tabular}{|c|c|c|}
\hline URL & Provider & Purpose \\
\hline $\begin{array}{l}\text { http://biotools.nubic.northwestern.e } \\
\text { du/OligoCalc.html }\end{array}$ & Northwestern University, USA & Pimer properties analysis \\
\hline $\begin{array}{l}\text { https://www.ncbi.nlm.nih.gov/pub } \\
\text { med/ }\end{array}$ & $\begin{array}{l}\text { National Institute of Health, } \\
\text { Bethesda, USA }\end{array}$ & Literature \\
\hline http://www.uniprot.org/ & $\begin{array}{l}\text { European Bioinformatic Institute } \\
\text { (EMBL-EBI), Swiss Institute of } \\
\text { Bioinformatic (SIB), Protein } \\
\text { Information Resource (PIR) }\end{array}$ & $\begin{array}{l}\text { Protein information and } \\
\text { analysis }\end{array}$ \\
\hline
\end{tabular}


http://www.insilico.uni-

duesseldorf.de/Lig_Input.html

https://www.expasy.org/

http://wlab.ethz.ch/protter/start/

http://subtiwiki.uni-goettingen.de/
Düsseldorf University

Swiss Institute of Bioinformatics (SIB)

Wollscheid Laboratory

General Microbiology department, Göttingen University ratio

calculation in PCR

Protein topology prediction

Protein topology prediction

Literature about B. subtilis

\subsubsection{Softwares}

Table XX Softwares

\begin{tabular}{l|l|l}
\hline Software & Provider & Purpose \\
\hline $\begin{array}{l}\text { Microsoft Office 2010 } \\
\text { ImageLab }{ }^{\text {TM }} \text { Software }\end{array}$ & $\begin{array}{l}\text { Microsoft Inc. } \\
\text { BioRad } \\
\text { Clarivate } \\
\text { analytics } \\
\text { EndNote }\end{array}$ & $\begin{array}{l}\text { Primer properties analysis } \\
\text { Image processing }\end{array}$ \\
SigmaPlat software, & References \\
Inc & Plotting \\
ChemoStar Imager & $\begin{array}{l}\text { Intas } \\
\text { NIH }\end{array}$ & Image processing \\
Amage J 1.49p & Zeiss & Image processing \\
GenioVision Rel. 4.8 & Biomatters Ltd & Bioinformatics \\
\hline \hline
\end{tabular}

\subsection{Abbreviations}

Tabla XXI Abbreviations

\section{General abbreviations}

\begin{tabular}{l|l}
\hline$\%(\mathrm{vol} / \mathrm{vol})$ & $\%$ (volume/volume) \\
\hline $\mathrm{Amp}^{\mathrm{r}}$ & Ampicillin Resistance \\
\hline APS & Ammonium persulfate \\
\hline $\mathrm{ATP}$ & adenosine triphosphate \\
\hline $\mathrm{Au}$ & Absorption units \\
\hline Biovar. & Biovariety \\
\hline BSA & Bovine Serum Albumin \\
\hline
\end{tabular}




\begin{tabular}{|c|c|}
\hline CAA & Casaminoacids \\
\hline CE & Crude Extract \\
\hline $\mathrm{Cm}^{\mathrm{r}}$ & Chloramphenicol resistance \\
\hline $\mathrm{dH}_{2} \mathrm{O}$ & Deionized water \\
\hline DNA & Deoxyribonucleic acid \\
\hline DNase & DNA nuclease \\
\hline dNTP & desoxyribonucleoside- triphosphate \\
\hline DTT & Dithiothreitol \\
\hline EDTA & N, N, N', N'- ethylenediaminetetraacetic acid \\
\hline $\mathrm{Em}^{\mathrm{r}}$ & Erithromycin resistance \\
\hline FDA & Food and Drug Administration \\
\hline Fig. & figure \\
\hline FT & Flowthrough \\
\hline Fwd & forward \\
\hline IPTG & Isopropyl thiogalactopyranoside \\
\hline $\mathrm{Km}^{\mathrm{r}}$ & Kanamycin Resistance \\
\hline LAB & Lactic Acid Bacteria \\
\hline LB & Luria Bertani \\
\hline Max & maximum \\
\hline MWM & Molecular Weight Marker \\
\hline NAD & Nicotinamide Adenine Dinucleotide (oxidized) \\
\hline NADH & Reduced Nicotinamide Adenine Dinucleotide \\
\hline $\mathrm{Ni}^{2+}-\mathrm{NTA}$ & nickel-nitrilotriacetic acid \\
\hline NICE & Nisin Control Expression System \\
\hline NIH & National Institute of Health \\
\hline Nis & Nisin \\
\hline NTP & ribonucleoside triphosphate \\
\hline ODx & Optical density, measured at the wavelength $\lambda=\mathrm{x} n \mathrm{~nm}$ \\
\hline $\mathrm{P}$ & promoter \\
\hline PBS & Phosphate buffer saline \\
\hline PCR & Polymerase Chain Raction \\
\hline $\mathrm{pH}$ & Power of hydrogen \\
\hline rbs & Ribosome Binding Site \\
\hline rpm & Revolutions per minute \\
\hline $\mathrm{Rv}$ & Reverse \\
\hline
\end{tabular}




\begin{tabular}{|c|c|}
\hline SDS & sodium dodecyl sulfate \\
\hline SDS-PAGE & polyacrylamide gel electrophoresis with SDS \\
\hline Sn & Supernatant \\
\hline Spp & Species \\
\hline TEMED & N,N,N’,N'- tetramethylethyldiamine \\
\hline Tris & tris(hydroxymethyl)amino- methane \\
\hline WT & wild type \\
\hline $\mathrm{X}-\mathrm{Gal}$ & 5-bromo-4-chloro- indolylgalactopyranoside \\
\hline \multicolumn{2}{|l|}{ Units } \\
\hline A & Ampere \\
\hline bp & base pairs \\
\hline $\mathrm{Da}$ & Dalton \\
\hline g & gram \\
\hline $\mathrm{h}$ & hour \\
\hline Lt. & liter \\
\hline $\mathrm{m}$ & meter \\
\hline $\mathrm{M}$ & molar \\
\hline $\min$ & minute \\
\hline Mol & $\mathrm{mol}$ \\
\hline V & volt \\
\hline${ }^{\circ} \mathrm{C}$ & degree Celsius \\
\hline \multicolumn{2}{|l|}{ Prefixes } \\
\hline K & kilo \\
\hline $\mathrm{m}$ & milli \\
\hline$\mu$ & micro \\
\hline $\mathrm{n}$ & nano \\
\hline \multicolumn{2}{|c|}{ Nucleosides } \\
\hline A & adenine \\
\hline $\mathrm{C}$ & cytosine \\
\hline G & guanine \\
\hline $\mathrm{T}$ & thymine \\
\hline $\mathrm{U}$ & uracil \\
\hline
\end{tabular}

\section{Amino acids nomenclature}

\begin{tabular}{l|l}
\hline A & Ala \\
\hline C & Cys \\
\hline
\end{tabular}




\begin{tabular}{l|l}
\hline D & Asp \\
\hline E & Glu \\
\hline F & Phe \\
\hline G & Gly \\
\hline H & His \\
\hline I & Ile \\
\hline K & Lys \\
\hline L & Leu \\
\hline M & Met \\
\hline N & Asn \\
\hline P & Pro \\
\hline Q & Gln \\
\hline R & Arg \\
\hline S & Ser \\
\hline T & Thr \\
\hline V & Val \\
\hline W & Trp \\
\hline Y & Tyr \\
\hline \hline & \\
\hline
\end{tabular}




\section{Curriculum vitae}

\section{PERSONAL INFORMATION}

Name: Ingrid María Quintana

Date of birth: March $27^{\text {th }} 1990$

Place of birth: Rosario, Santa Fe (Argentina)

Nationality: Argentina

\section{EDUCATION AND TRAINING}

\section{9/14 - 04/18 PhD in Molecular Biosciences and Biomedicine}

Universidad Nacional de Rosario (UNR - Argentina) - Georg-AugustUniversität (Göttingen - Germany)

Title of thesis: Cyclic di-nucleotide monophosphate cyclase in Firmicutes: from basic to practical approach. Instituto de Biología Molecular y Celular de Rosario (IBR - CONICET) - Institute for Genetics and Microbiology (Department of General Microbiology (Georg-August-Universität)

\section{4/13 - 08/14 PhD in Biological Sciences}

Universidad Nacional de Rosario (UNR - Argentina)

Title of thesis: Cyclic di-nucleotide monophosphate cyclase in Firmicutes: from basic to practical approach. Instituto de Biología Molecular y Celular de Rosario (IBR - CONICET)

\section{4/08 - 03/13 Licenciatura en Biotecnología (B.Sc. in Biotechnology)}

Facultad de Ciencias Bioquímicas y Farmacéuticas - Universidad Nacional de Rosario

\section{PUBLICATIONS}

Martino GP, Quintana IM, Espariz M, Blancato VS, Magni C. Aroma compounds generation in citrate metabolism of Enterococcus faecium: Genetic characterization of type I citrate gene cluster. Int J Food Microbiol. 2016 Feb 2;218:27-37.

Martino GP, Quintana IM, Espariz M, Blancato VS, Gallina Nizo G, Esteban L, Magni C. Draft Genome Sequences of Four Enterococcus faecium Strains Isolated from Argentine Cheese. Genome Announc. 2016 Feb 4;4(1). pii: e01576-15. 
Quintana IM, Grubek Z., Gibhardt J., Lee V. T., Hammer E., Commichau F. M., Stülke J., and Magni C. The KupA and KupB proteins of Lactococcus lactis IL1403 are c-di-AMP receptor proteins responsible for potassium uptake. (2018) Applied and Environmental Microbiology. Sent.

Quintana IM, Espariz M., Stülke J., Villar S., González F., Pacini F., Cabrera G., Bontempi I., Procheto E., Perez A. R., Marcipar I., Blancato V., and Magni C. Genetic engineering of Lactococcus lactis co-producing antigen and the mucosal adjuvant 3' 5' - cyclic di adenosine monophosphate (c-di-AMP) to design a mucosal vaccine prototype. (2018) Frontiers in Microbiology. Sent. 